C. R. Smith, Secretary

NATIONAL BUREAU OF STANDARDS

A. V. Astin, Director

\title{
QUANTITATIVE ELECTRON PROBE MICROANALYSIS
}

\author{
Proceedings of a Seminar held at the \\ National Bureau of Standards. \\ Gaithersburg, Maryland, \\ June 12-13, 1967 \\ K. F. J. Heinrich, Editor \\ Institute for Materials Research \\ National Bureau of Standards \\ Washington, D.C. 20234
}

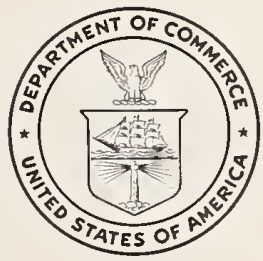

National Bureau of Standards Special Publication 298

Issued October 1968 


\begin{abstract}
A seminar was held at the National Bureau of Standards in June 1967 to examine critically the factors involved in quantitative electron-probe microanalysis. Major consideration was given to proposed methods for data evaluation, and to requirements for further work in theory, in measurement, and in the preparation of standards. This volume contains a series of invited papers which formed the basis of discussion at the seminar. Topics covered include corrections for the atomic number effect, for x-ray absorption, and for fluorescence by characteristic lines and the continuum. The various ways to derive a simplified model of the complex electron-target interaction are critically analyzed by several authors, and the accuracy of several proposed methods is compared by error histograms constructed on the basis of hundreds of analyses of materials of known composition. Applications to the analysis of biological specimens and to problems of stereometric analysis are also discussed.
\end{abstract}

Key Words: Atomic number effect, biological specimens, electron probe microanalysis, electron scattering, fluorescence by the continuum, quantitative analysis, stereometric analysis, $\mathrm{x}$-ray absorption, x-ray emission.

Library of Congress Catalog Card No. : 68-60068 


\section{FOREWORD}

Development and improvement of measurement techinques for the characterization of materials is a prime responsibility of the Institute for Materials Research of the National Bureau of Standards. The Institute carries out this responsibility by serving as a focal point for the development of measurement methodology and by encouraging the dissemination of relevant information.

The seminar "Quantitative Electron Probe Microanalysis" held June 12-13, 1967 and documented by this book is typical of meetings held by the Institute at the National Bureau of Standards to examine critically an important area of measurement related to materials. In this seminar groups of experts were invited to bring the latest thinking to bear on a problem area of basic importance to materials science.

Kurt F. J. Heinrich of our staff was chairman of the seminar. Some 25 participants from the United States and five other countries were in attendance. Papers were submitted in advance by the participants to form a basis for discussion. These papers, with some revisions after the seminar, constitute the subject matter of this book. Those interested in the refinement of quantitative measurement in this fruitful field of microanalysis will find this book to be relevant and quite informative.

John D. Hoffman, Director

Institute for Materials Research. 



\section{PREFACE}

Electron probe microanalysis is extensively used as a research tool in a wide range of scientific disciplines, including metallurgy, solid state physics, mineralogy, and biology. Scientists in several laboratories at locations distributed over the world are seeking to improve our knowledge of the physical bases and the quantitative aspects of this analytical technique. To stimulate this research, it is important to establish and maintain free interchange of ideas among the investigators. It is equally important to render the results of the research activity accessible to those whose interest in microprobe analysis is more recent or incidental.

To provide the necessary means of communication among investigators of quantitative electron probe microanalysis, a seminar on this subject was held at the National Bureau of Standards, Gaithersburg, Maryland, in June 1967. The aims and attainments of the seminar are described in some detail by L. S. Birks in the Introduction. To assist in the discussion, several participants prepared papers in advance on those aspects of quantitative analysis which were of particular interest to them. This book consists of the discussion papers, brought up-to-date after the seminar by the authors and the editor. We have also included a paper by Professor I. B. Borovskii and V. I. Rydnik, who were not participants in the seminar. Professor Borovskii visited our Institute a few days after the seminar, and he agreed to contribute this paper which was translated into English at the National Bureau of Standards.

In view of the variety of approaches it was logical and desirable that several subjects should be discussed, in different ways, by more than one author. In order to conserve the originality of the contributions, we have endeavored to keep the editorial changes (including nomenclature) to a minimum. Whatever consensus can be observed is not due to the pressure of the editor but to the fact that, at last, an increasing convergence of opinions on the subject is taking place.

We are indebted to Mrs. Rosemary S. Maddock and to her co-workers for the editorial assistance which made this book possible. We also thank Mrs. Mary Ann Morris Giles who very efficiently took care of local arrangements for the seminar and participated in the editing of the manuscripts. 



\section{CONTENTS}

Page

Foreword .................... iii

INTRODUCTION .................... 1

Birks, L. S.

QUANTITATIVE ELECTRON PROBE MICROANALYSIS: A PROGRESS REPORT ....... Heinrich, K. F. J.

SOME PROBLEMS WITH QUANTITATIVE ELECTRON PROBE MICROANALYSIS ......... 13 Philibert, J. and Tixier, R.

THE THEORY OF QUANTITATIVE ELECTRON PROBE MICROANALYSIS .......... 35

Borovskii, I. B. and Rydnik, V. I.

ON THE STRUCTURE OF FORMULAS FOR QUAN-

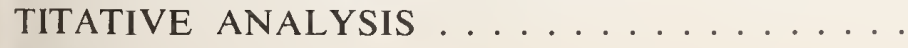
Criss, $\mathbf{J}$.

COMPUTATIONAL METHODS FOR X-RAY EMISSION FROM TARGETS EXCITED BY ELECTRONS

Brown, D. B.

THE CHOICE OF MODELS FOR ELECTRON SCATTERING AND DECELERATION FOR ELECTRON

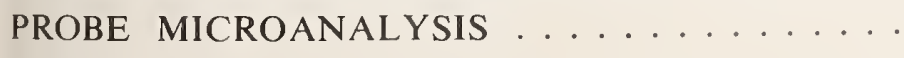
Mulvey, T. 


\section{CONTENTS - Continued}

PROGRESS IN THE CORRECTION FOR THE ATOMIC NUMBER EFFECT .......... Poole, D. M.

'THE CALCULATION OF STOPPING POWER AND BACKSCATTER EFFECTS IN ELECTRON PROBE MICROANALYSIS . . . . . . . . . . .

Duncumb, P. and Reed, S. J. B.

SCATTERING OF ELECTRONS IN METALLIC

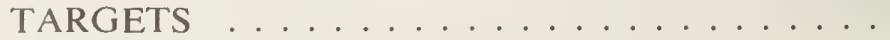

Murata, K., Shimizu, R. and Shinoda, G.

ABSORPTION EDGE EFFECTS IN ELECTRON PROBE ANALYSIS . . . . . . . . . . . . . . . 189

Nagel, D. and Criss, J.

FLUORESCENCE EXCITED BY THE CONTINUUM .

Hénoc, J.

QUANTITATIVE EVALUATION METHODS FOR ALLOY MICROSTRUCTURES BY MICROPROBE

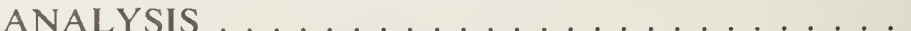

Dörfler, G.

SOME ASPECTS OF THE MICROPROBE ANALYSIS OF BIOLOGICAL SPECIMENS .......... Hall, T. 


\title{
QUANTITATIVE ELECTRON PROBE MICROANALYSIS
}

\section{INTRODUCTION}

\author{
L. S. BIRKS \\ Naval Research Laboratory, \\ Washington, D. C.
}

A seminar on quantitative Electron Probe Analysis was held at the National Bureau of Standards, Gaithersburg, Md., in June 1967. About twenty experienced people from six countries were invited for two days of extended informal discussions on mathematical models for calculations, values of parameters, selection of standards, and measuring techniques. Backgrounds and interests of the attendees varied from more applied science such as biology, metallurgy, and mineralogy to the less applied fields of mathematics, physics, and chemistry.

The objective was for the attendees to bring each other up to date on the most current attitudes and methods in electron probe analysis in the various countries. The approach was to consider and discuss questions such as "Where are we today? Where should we be heading? Which areas of work should be given priority? What are the chances of achieving accuracy of $1 \%$ for major constituents and what improvement in theory or experiment will be necessary to achieve such accuracy?"

Actually, there were no formal presentations made at the seminar but the papers in this volume were submitted by several of the attendees as background information for the discussions. They vary considerably in coverage and approach. Some of them draw together previously published work to present a comprehensive review. Others assess the current situation in an area by considering the questions rather than giving answers. Still others suggest new concepts or report new measurements. Unfortunately, the printed papers cannot recapture the full flavor of the seminar but that would not be possible even with a direct transcript of the discussions. Hopefully they will be useful as a guide to the areas covered for those interested parties who could not be present at the seminar. 
In the discussions there was no attempt to reach a consensus in any of the subject areas. However, a few remarks may be made on general attitudes:

1. The need for dependable experimental measurements of absorption coefficients, fluorescent yields, backscatter coefficients, ionization cross-sections and other parameters is becoming more critical as more workers come to rely on calculation methods for quantitative analysis.

2. It may be necessary to go back to the physical models and examine such fundamental concepts as electron retardation to make sure the forms and values used are really applicable to electron probe analysis and to estimate what kinds of errors have been introduced by which approximations.

3. There are continuing needs for approximate calculation methods which can be performed with slide rules or desk calculators as well as for more elaborate computer programs. Attempts should be made to predict limits of accuracy of the approximate methods by comparison with the more elaborate calculations for some well chosen types of specimens.

4. Fluorescence by the continuum has been improperly overlooked or neglected in cases such as $\mathrm{Cu}-\mathrm{Au}$ where it may change the calculated composition by three to five percent of the amount present. Present computer programs make calculation of the continuum fluorescence feasible when large differences in atomic number of the components indicate it cannot be neglected.

5. Preparation and testing of comparison standards, both homogeneous and inhomogeneous, should be pursued vigorously to augment and verify the mathematical calculation methods.

6. Valence state and absorption fine structure are of increasing importance in quantitative analysis of low atomic number specimens such as biologicals. Parameters for such specimens are more uncertain than for metals and minerals but fortunately the acceptable limits of error in quantitative analysis of biologicals are even less stringent. It would be of great interest to investigate if the valence state in compounds of interest remains unchanged when the specimen is bombarded by electrons.

Because of the varied interests of the participants, one of the original objectives was not reached or even approached. That is, there was no agreement on any kind of priority list of problems to be attacked. The mathematicians left still feeling that setting up more 
rigorous expressions for absorption, scattering, etc. were the most pressing problems; metallurgists and physicists still planned rigorous pursuit of solid-state reactions by experimental means; chemists still contended that preparation of reliable homogeneous standards and calibration curves was the first order of business. Nevertheless, the exchange of views was valuable and informative and an excellent way to catch up on all phases of quantitative electron probe analysis. 



\section{QUANTITATIVE ELECTRON PROBE MICROANALYSIS: A PROGRESS REPORT

\author{
KURT F. J. HEINRICH
}

Spectrochemical Analysis Section, Analytical Chemistry Division,

National Bureau of Standards

\section{Introduction}

Since the appearance of the first publications of Castaing $[1,2]$ electron probe microanalysis has been applied to an ever increasing variety of specimens. The energy range of $x$-rays used in this technique has also been extended considerably. In this process many difficulties arose in the quantitative determination of the specimen composition. Hence, many investigators have spent great efforts to render quantitative microprobe analysis more effective. We hope that the present NBS publication will give the reader a balanced view of the present state of art as well as of the areas in which further investigations are required.

Solutions to the present problems are sought by a concerted attack from both the theoretical and the empirical points of view. Neither of these viewpoints is at present entirely satisfactory. Theory does not as yet describe unequivocally the electron-target interaction - and the subsequent $\mathrm{x}$-ray emission - with the accuracy required for quantitative analysis. Hence, empirical adjustments are necessary to make models agree with the experiments. On the other hand, a fully empirical treatment of the problem is impractical in view of the excessive number of factors affecting the intensity of $\mathrm{x}$-ray emission from a complex target. The lack of a sufficient number of reliable standards further hampers the testing of proposed procedures. However, in view of the progress made in recent years, it can be expected that, with a sustained effort, the remaining gap between theory and experiment will be rapidly closed.

It should not go unmentioned that much valuable information can be obtained in practice through qualitative and semiquantitative analysis, in particular by applying scanning techniques. This area has been reviewed elsewhere [3] and will not be considered here. 


\section{Theory of Quantitative Analysis}

Quantitation is based upon the comparison of primary characteristic $x$-ray intensities emitted from the specimen and the standard, respectively. To predict these intensities, we must know the ratio of the $x$-ray intensities produced within the specimen and the standard, respectively, as well as the distributions with depth of the $x$-ray generation. Since the ionization cross-sections of the atomic levels producing the $\mathrm{x}$-ray photons of interest vary with the energy of the exciting electrons, a workable theory of microprobe quantitation must provide, in the first place, a quantitative description of the electron fluxes and energy distributions as a function of the depth of penetration. (See paper 10, by Murata, Shimizu and Shinoda.) Diverse aspects of this problem are considered in the communications included in this volume. It is customary to treat separately the variation of intensities generated within the specimen as a function of target composition (atomic number effect). This subject is treated in detail in the papers 3 to 10 . The knowledge of the emission distribution in depth permits, in turn, calculating of the attenuation of the emerging radiation. Therefore (see Philibert, paper 3), we submit the measured emerging intensity ratios to an "absorption correction" and to an "atomic number correction". However, as pointed out by Brown (paper 6), this approach, involving two separate corrections to the primary intensities, might not be necessary in future procedures.

Besides the primary characteristic radiation, the specimen also emits fluorescent radiation, which may have been excited by both characteristic radiation of other elements and by continuous radiation (Bremsstrahlung). The theory of correction for fluorescence excited by characteristic rays $[1,4]$ is fairly well known and generally applied although further critical experiments in this area are desirable. The excitation by continuous radiation, however, is far more complex [5]. Since the need for correcting for this effect is now becoming more apparent, its discussion by $\mathrm{J}$. Hénoc (paper 12) is a welcome contribution to this publication.

The continuous radiation falling within the spectrometer acceptance is part of the background which must be subtracted from the measured intensities. Philibert (paper 3) suggests that care must be taken in determining the appropriate background level. Critical studies in this area would also be useful. 


\section{Experimental Support}

\section{A. TEST ANALYSES}

While many investigators have published the results of analyses of presumably well characterized test specimens, Poole and Thomas $[6,7]$ were the first to critically evaluate the effectiveness of diverse proposed correction procedures, using a large number of measurements performed on such specimens by various laboratories. This is extended to an even larger number of test specimens and procedures in Poole's present contribution (paper 8). Similar error distributions are also included in Duncumb and Reed's paper (paper 9).

The effective performance of test analyses is a crucial test for any proposed analytical procedure; it is difficult, however, to locate unequivocally the sources of errors by this method. In a study of the data used by Thomas [6], Heinrich [8] showed evidence that the most serious failures are not due to the atomic number correction. There is a strong indication that significant errors in this group can be attributed to excessive absorption of x-rays, to fluorescence by characteristic lines, and to errors in the chemical analysis of some test specimens. To improve the value of such test analyses, standard reference materials of tested homogeneity and accurately determined composition will have to be prepared.

\section{B. PARTIAL TESTS}

In view of the complexity of the target events it is important to provide tests that permit the locating of sources of error in the correction scheme. To cite a few examples, an electron distribution model that fails to predict the correct values of the electron backscatter coefficients must be expected to fail to predict the correct $\mathrm{x}$-ray production as well. Therefore, the improved knowledge of the electron backscatter coefficients $[9,10]$ permits testing such models; an even more sensitive test could be established if the energy and angular distributions of backscattered electrons were also redetermined reliably.

It is interesting to note in this context that some theoretical expressions for the electron backscatter coefficient (e.g., Borovskii and Rydnik, paper 4) predict a dependence upon the ratio of atomic weight to atomic number, while other models do not contain a term involving this ratio. The backscatter coefficients determined experimentally by Heinrich [10] exhibit such a dependence, in qualitative agreement with Borowskii's prediction (Fig. 1). 


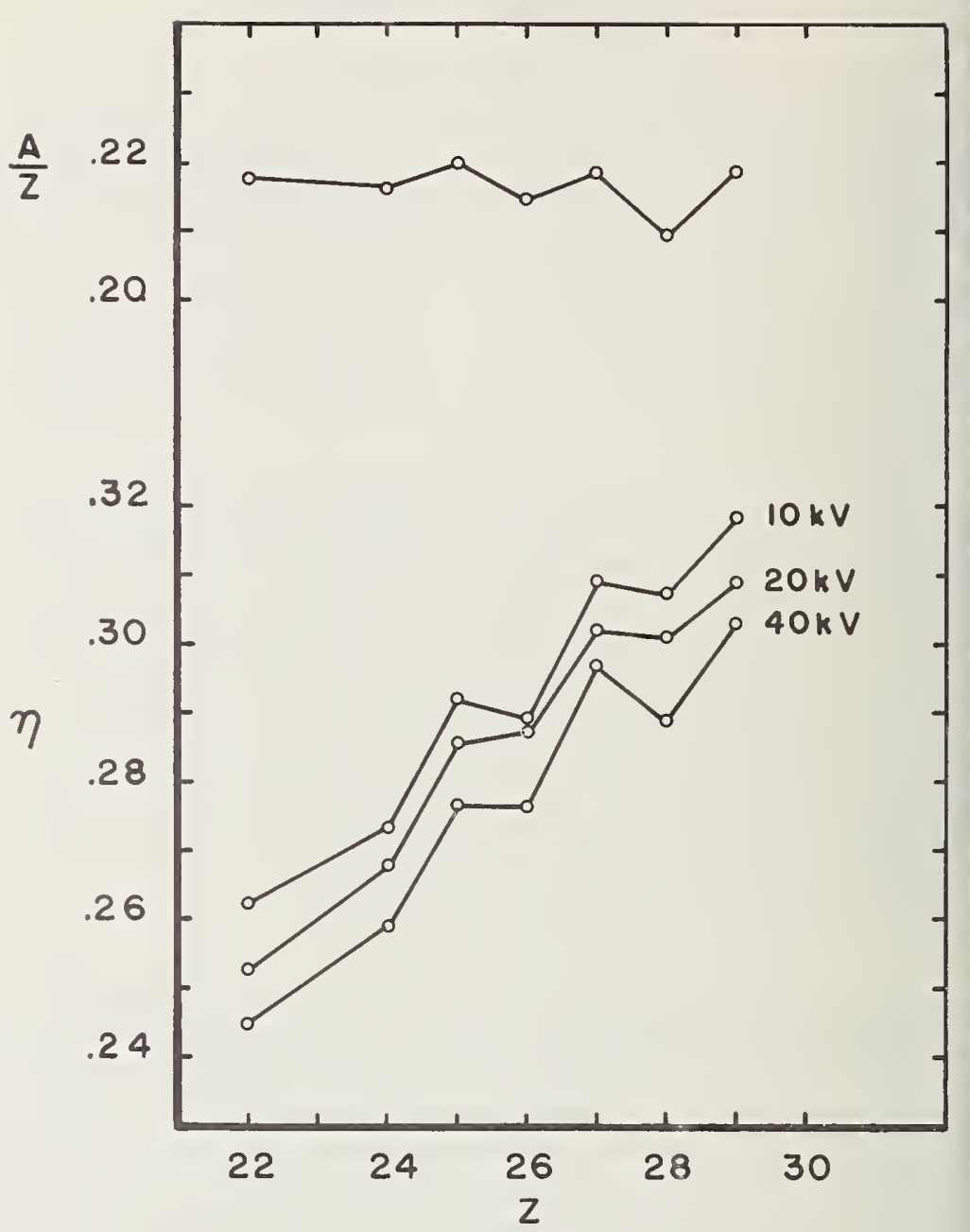

Figure 1. Electron backscatter coefficients and $A / Z$ ratios for elements 22-29.

The most important partial tests are those involving $x$-ray emission. These include the following experiments:

a. Determination of the distribution in depth of emitted x-ray intensities by the tracer method. Most work in this area concerns primary emission $[2,11]$; but $\mathbf{J}$. Brown has also studied by this technique the depth distribution of fluorescent emission [12]. 
b. Determination of absorption of emitted $\mathrm{x}$-rays as a function of the emergence angle $[1,13]$.

c. Measurement of secondary emission by the undiffused-couple method $[14,15]$.

d. Determination of secondary emission caused by the continuum, by the aluminum-layer method $[2,5]$.

e. Experimental determination of the backscatter loss [16].

f. Measurement of the variation of $x$-ray emission as a function of accelerating voltage [17].

These experiments have been indispensable for the establishment of methods for correcting electron probe measurements. It must be added, however, that the experimental evidence available at present is insufficient from both the qualitative and quantitative point of view. The results of some attenuation experiments are available only in form of graphs difficult to read with the required precision, and it is particularly difficult to separate the effects of primary emission from those of emission by the continuum. There is no experimental evidence to speak of concerning the fluorescence excitation of K-lines by L-lines and vice versa, and even the case of $\mathrm{K}-\mathrm{K}$ excitation is not sufficiently documented, so that it is impossible to test efficiently the models proposed for this case. Moreover, practically all these experiments were performed on pure elements, so that the emission from composite targets, observed in the microprobe, can only be calculated using untested assumptions. ${ }^{1}$ More, and more precisely executed and documented, experiments will have to be performed to provide more solid bases of comparison with theoretical speculation.

\section{Errors of Input Parameters}

The effects of errors in the input parameters for correction models have not been fully recognized in the past. An analysis or error propagation in the absorption and fluorescence (by characteristic lines) corrections was performed recently [18]. This work shows that uncertainties in the values of $x$-ray mass attenuation coefficients, of the emergence angle, and of the fluorescent yield can seriously impair the accuracy of analysis, even if the model itself is

${ }^{1}$ Furthermore, there is practically no information available concerning the effects of electron beams impinging upon the specimen at an angle other than normal; hence the theory of analysis with such a geometry is not well founded at the present time. 
satisfactory. ${ }^{2}$ In the case of the absorption correction, such effects can be minimized by a careful choice of the conditions of the measurement (i.e., using a high emergence angle and low excitation voltages). Similarly, a study of the validity of the assumptions frequently used in calculating electron scattering and deceleration is still needed. To mention only a few examples, the limitations of Bethe's law, particularly at low electron energies, should be investigated, as well as the effects of uncertainties in the ionization cross-sections.

A major difficulty in such investigations is that the necessary experiments as a rule require delicate and complex specimen preparation techniques, or $\mathrm{x}$-ray instrumentation other than the electron probe. The involved expenses in time and money can hardly be avoided, however, if the proper balance between theory and experiment is to be maintained.

\section{The Application of Electron Probe Microanalysis}

When microprobe analysis is applied to a variety of specimens, we find many difficulties not considered in most conventional analytical schemes. New problems arise, for instance, with specimens in which abrupt concentration changes arise over short distances, such as inclusions $[14,15]$. This problem has been discussed by Philibert (paper 3). Complications may also arise in non-conductive specimens, in the analysis of low atomic number elements, and in cases where chemical effects upon wavelength spectra (wavelength shifts, band-shape changes) become apparent. The analysis of thin films has been recently discussed by Hutchins [19] and by Colby [20].

Critical studies of the effects of specimen preparation were published recently by Picklesimer and Hallerman [21] and by Yakowitz [22]. Of great interest are also the experiments with specimen etching performed by Dörfler et al. [23]. These authors show that under carefully controlled conditions light etching of the specimen is frequently beneficial, contrary to common belief.

The analysis of soft biological tissue is a field of great potential importance. The special problems of specimen preparation, measurement, and interpretation of the results are discussed in the

${ }^{2}$ The communication of Nagel and Criss (paper 11) dealing with the uncertainty of mass attenuation coefficients in regions near the absorption edges is an important contribution in this field. 
contribution by $\mathrm{T}$. Hall (paper 14). The questions treated by this author will also be of interest in the analysis of non-biological organic specimens (plastic material, photographic emulsions, textiles, etc.). Another area in which further work is needed is the analysis of particulate material, such as mineral powders and atmospheric particles.

The contribution of Dörfler (paper 13) deals with quantitative phase analysis of heterogeneous specimens. This is an aspect of quantitative analysis which has not been given extensive and systematic attention until now. Yet, it offers unsuspected new applications of the microprobe to quantitative metallography and to analogous phase distribution studies in mineralogy.

Instrumental developments which are presently under study will eventually permit the quantitative characterization of an entire specimen area. Such techniques will combine the characteristics of quantitative microprobe analysis, of scanning electron probe analysis, and of phase analysis. To reach this goal, extensive use of digital computers will be made in the programming and evaluation of the analytical experiment. While many difficulties still stand in the way to this "total microanalysis", the possibility of such an exciting technique should stimulate the investigator in his present efforts.

\section{References}

[1] Castaing, R., Doctoral Thesis, University of Paris, 1951.

[2] Castaing, R., and Descamps, J., J. Phys. Radium 16, 304 (1955).

[3] Heinrich, K. F. J., NBS Tech. Note 278, 1967.

[4] Reed, S. J. B., Brit. J. Appl. Phys., 913 (1965).

[5] Hénoc, J., Maurice, F., Kirianenko, A., Commissariat a l'Energie Atomique, Centre d'Etudes Nucléaires de Saclay, France, Report CEA - R 2421 (1964).

[6] Thomas, P. M., Atomic Energy Research Establishment, Harwell, England, Report AERE - R 4693 (1964).

[7] Poole, D. M), and Thomas, P. M., "The Electron Microprobe", Eds. T. D. McKinley, K. F. J. Heinrich, and D. B. Wittry (J. Wiley and Sons, Inc., New York, 1966) p. 269.

[8] Heinrich, K. F. J., "Advances in X-Ray Analysis", Vol. 11, (Plenum Press, New York, 1968). To appear.

[9] Bishop, H. E., in "X-Ray Optics and Microanalysis", Eds. R. Castaing, P. Descamps, and J. Philibert, (Hermann, Paris, 1966) p. 153.

10] Heinrich, K. F. J., in "X-Ray Optics and Microanalysis", Eds. R. Castaing, P. Descamps, and J. Philibert, (Hermann, Paris, 1966) p. 159.

[11] Castaing, R., and Hénoc, J., in "X-Ray Optics and Microanalysis", Eds. R. Castaing, P. Descamps, and J. Philibert, (Hermann, Paris, 1966) p. 120. 
[12] Brown, J. D., Doctoral Thesis, University of Maryland, 1966.

[13] Green, M., in "X-Ray Optics and X-Ray Microanalysis", Eds. H. H. Pattee, V. E. Cosslett, A. Engström (Academic Press, New York, 1963) p. 361.

[14] Reed, S. J. B., and Long, J. V. P., in "X-Ray Optics and X-Ray Microanalysis", Eds. H. H. Pattee, V. E. Cosslett, A. Engström (Academii Press, New York, 1963) p. 317.

[15] Maurice, F., Seguin, R., and Hénoc, J., in "X-Ray Optics and Microanalysis", Eds. R. Castaing, P. Descamps, and J. Philibert (Hermann, Paris, 1966) p. 357.

[16] Castaing, R., and Derian, J. C., in "X-Ray Optics and Microanalysis", Eds. R. Castaing, P. Descamps, and J. Philibert (Hermann, Paris, 1966) p. 193.

[17] Green, M., in "X-Ray Optics and X-Ray Microanalysis", Eds. H. H. Pattee, V. E. Cosslett, A. Engstrōm (Academic Press, New York, 1963) p. 185.

[18] Yakowitz, H., and Heinrich, K. F. J., Microchim. Acta (1968) in press.

[19] Hutchins, G., in "The Electron Microprobe", Eds. T. D. McKinley, K. F. J. Heinrich, and D. B. Wittry (J. Wiley and Sons, Inc., New York, 1966) p. 390.

[20] Colby, J. W., in "Advances in X-Ray Analysis", Vol. 11 (Plenum Press, New York, 1968). To appear.

[21] Picklesimer, M. L., and Hallerman, G., U) S. Atomic Energy Commission, Report ORNL-TM-1591 (1966).

[22] Yakowitz, H., ASTM Tech. Publ. 430, (American Society for Testing and Materials, Philadelphia, 1963) p. 383.

[23] Dörfler, G., Blöch, R., and Plöckinger, E., Arch. f. d. Hüttenw. 37, 375 (1966). 


\title{
SOME PROBLEMS WITH QUANTITATIVE ELECTRON PROBE MICROANALYSIS
}

\author{
J. PHILIBERT and R. TIXIER \\ Research Institute of the French Ferrous Metallurgy (IRSID), \\ St. Germain-en-Laye, France
}

\begin{abstract}
The main lines of correction calculations are briefly reviewed, according to the ZAF scheme-where $\mathrm{Z}$ represents the atomic number effect, $A$ the absorption, and $F$ the fluorescence. The atomic number correction is discussed in detail and its, importance in the case of thin specimens analysis is emphasized.
\end{abstract}

\section{Introduction}

Electron probe' microanalysis has been frequently considered as an absolute method, since it gives directly the mass concentration of an element $\mathrm{A}$ in a complex target $\mathrm{ABC} \ldots$ :

$$
\mathrm{C}_{A}=\left(\mathrm{I}_{A} / \mathrm{I}(\mathrm{A})\right)
$$

with the usual notation.

This relation is of course an approximate one, and the first question which arises is as follows: what kind of intensities are to be considered in relation (1)?

A careful distinction has to be made between generated intensities and emerging intensities.

Let us call $k_{A}$ the ratio of intensities of radiations (i.e., $K \alpha$ ) generated within both targets (specimen and standard)

$$
\mathrm{k}_{A}=\left(\mathrm{I}_{A} / \mathrm{I}(\mathrm{A})\right)
$$

The theory has to derive the relation between $\mathrm{k}_{A}$ and $\mathrm{C}_{A}$.

In Castaing's "first approximation", the relationship is simply:

$$
\mathrm{k}_{A}=\mathrm{C}_{A}
$$

while the Castaing "second approximation" takes the form

$$
\mathrm{k}_{\mathrm{A}}=\frac{\alpha_{\mathrm{A}} \mathrm{C}_{\mathrm{A}}}{\sum_{\mathrm{j}} \alpha_{\mathrm{j}} \mathrm{C}_{\mathrm{j}}}
$$


A more exact approximation which may be easily derived, is:

$$
\mathrm{k}_{\mathrm{A}}=\mathrm{C}_{\mathrm{A}} \frac{(\mathrm{R} / \mathrm{S})_{\text {specimen }}}{(\mathrm{R} / \mathrm{S})_{\text {standard }}}
$$

$\mathrm{R}$ is the "backscattering factor" (loss of ionizations due to electron backscattering) and S is the "stopping power factor". Both factors depend on the electron retardation law and the ionization crosssection of $\mathrm{A}$ atoms. Moreover, $\mathrm{R}$ depends on the number and energy distribution of backscattered electrons.

The second formula to be derived relates the ratio $\mathrm{k}_{A}$ of generated intensities to the ratio $\mathrm{K}_{A}^{\theta}$ of the intensities $\mathrm{J}$ of the radiation emerging from the specimen and entering the spectrometer

$$
\mathrm{K}_{A}^{\theta}=\left(\mathbf{J}_{A} / \mathbf{J}(\mathrm{A})\right)
$$

Two effects contribute to the difference between $\mathrm{K}_{A}^{\theta}$ and $\mathrm{k}_{A}$. First of all, radiation is absorbed inside the target and secondly, fluorescent radiation is emitted with the same wavelength as the primary. Relationships (3), (4), or (5) are only valid for primary emissions.

Thus, the essential features of the calculations may be summarized as follows:

\begin{tabular}{|l|l|l|l|l|l|l|}
\hline $\begin{array}{l}\text { ratio of } \\
\text { total } \\
\text { emergent } \\
\text { intensi- } \\
\text { ties } \\
\mathrm{K}_{A}^{\theta}\end{array}$ & $\begin{array}{l}\text { fluores- } \\
\text { cence }\end{array}$ & $\begin{array}{l}\text { ratio of } \\
\text { primary } \\
\text { emergent } \\
\text { intensi- } \\
\text { ties } \\
\mathrm{k}_{A}^{\theta}\end{array}$ & $\begin{array}{l}\text { absorp- } \\
\text { tion }\end{array}$ & $\begin{array}{l}\text { ratio of } \\
\text { primary } \\
\text { generated } \\
\text { intensi- } \\
\text { ties } \\
\mathrm{k}_{A}\end{array}$ & $\begin{array}{l}\text { atomic } \\
\text { number } \\
\text { effect }\end{array}$ & $\begin{array}{l}\text { mass } \\
\text { concen- } \\
\text { tration }\end{array}$ \\
$\mathrm{C}_{A}$
\end{tabular}

The secondary emissions have to be subtracted from the total measured intensities $\left(\mathrm{K}_{A}^{\theta} \rightarrow \mathbf{k}_{A}^{\theta}\right)$. The primary emergent intensities have then to be corrected for the absorption effect $\left(\mathrm{k}_{A}^{\theta} \rightarrow \mathrm{k}_{A}\right)$ and finally the true concentration $\mathrm{C}_{A}$ is calculated from the ratio of primary generated intensities (atomic number effect).

This sequence may be expressed as the FAZ sequence. Conversely, by calculating the ratio of emergent intensities from the known (or assumed) concentrations, the ZAF sequence has to be followed. "Zafizing" is the usual method of calculation for 
establishing theoretical calibration curves, $\mathrm{K}_{A}^{\theta}$ versus $\mathrm{C}_{A}$ or $\mathrm{K}_{A}^{\theta} / \mathrm{C}_{A}$ versus $\mathrm{C}_{A}$ (or $\mathrm{K}_{A}^{\theta}$ ), or $\mathrm{K}_{A}^{\theta}-\mathrm{C}_{A}$ versus $\mathrm{K}_{A}^{\theta}$, while "Fazising" is generally employed for iterative calculations.

The importance of the order of the factors (namely FAZ or ZAF) should be noted. If the calculation stages are expressed as multiplicative factors, i.e.,

$$
\mathrm{k}_{\mathrm{A}}^{\theta}=\mathrm{K}_{\mathrm{A}}^{\theta} \cdot \mathrm{f}\left(\mathrm{C}_{\mathrm{i}}\right)
$$

and so on ..., the order of calculation is not significant. This is the case of many calculations, mainly with computers when iterative methods are used.

Nevertheless, experience seems to me to show that frequently one of the corrections is more significant than the others and its relative importance appears clearly when the right order of the sequence is respected. Generally speaking, every time the calculation cannot directly obey the general scheme, it is necessary to examine the physical significance of the processes involved, and the ZAF scheme remains the basis of such a critical investigation.

In developing general methods, homogeneous targets are assumed but in practice this assumption is often not justified. Analyses near boundaries (precipitates, diffusion, layers, ....) are very frequent, and a "blind" calculation becomes impossible. Here again a clear understanding of the physical processes involved (as shown by the ZAF scheme) is absolutely essential.

I would suggest that the case of non-homogeneous targets has not yet received all the care it deserves. The correction calculation method must be generally verified by looking at homogeneous targets, although platings of A/B type are also very useful from this point of view and can give useful data in connection with absorption or fluorescence effect: $\phi(\rho \mathrm{z})$ function, ratio of secondary to primary emission, etc. .. .). But in practice, the "microprobist" has to deal with boundaries, precipitates, and so on, so that each new measurement often appears as a "particular case" and does not enter the general scheme of calculations for which programs are available.

From this point of view, the analysis of small particles embedded in a more or less amorphous matrix has not yet received sufficient attention. Such is the case for extraction replicas in metallurgy, foreign particles in biological tissues, . . . The quantitative analysis of such specimens is very crucial in many fields of research; it has expanded widely with the developments of EMMA and similar 
instruments combining electron microscopy and microanalysis. General methods of calculation (absorption, atomic number) are lacking for such specimens and no precise tests have been made to determine whether the corrections are negligible or not.

\section{Discussion}

\section{A. ABSORPTION CORRECTION}

The analytical expression for the $f(\chi)$ functions has been widely employed for some years, in spite of the drastic assumptions used in its derivation, even with the "effective $\sigma$ " improvement suggested by Duncumb and Shields.

In the computer age however, the need for simple formulae is not as acute as during the slide rule period. It is thus to be hoped that the "collective model" on which the formula is based will be reconsidered in spite of the many difficulties likely to be encountered. The inaccuracies and the inadequacies of the physical laws involved (multiple scattering and diffusion, electron attenuation and retardation laws, etc.) have been pointed out in a series of papers by Cosslett and Thomas [11].

Notwithstanding the rather pessimistic conclusions of these authors, this description offers nevertheless several advantages, the most important being that a good derivation of the $\phi(\rho \mathrm{z})$ distribution function would enable the $\mathrm{Z}$ and $\mathrm{A}$ factors to be calculated in a single step.

\section{B. FLUORESCENCE CORRECTION}

I should like to present only two short remarks about these corrections:

(a) characteristic radiation: the well known Castaing [8] formula, with or without the improvements or the simplifications due to several authors (Birks, Reed, Wittry, . . .), applies first of all to $\mathrm{K}-\mathrm{K}$ excitation. The case of $\mathrm{L}-\mathrm{L}, \mathrm{K}-\mathrm{L}$, . . excitations have perhaps not yet been analyzed with the careful attention required. For instance, in his general paper, Reed (1965) [17] finally gives a numerical application only in the case of $K-K$ excitation. Furthermore, the absence of data on the fluorescence yield and the absorption jumps, together with the complexity of Coster-Kronig transitions, seem to frighten off fresh investigation in that field.

(b) continuous spectrum: I am afraid that people have too frequently claimed this correction to be negligible, without any 
calculations to ascertain the validity of such an assumption. In the case of $A / B$ interfaces, this fluorescence effect is known to be frequently quite important.

\section{ATOMIC NUMBER EFFECT}

With the assumption of a continuous energy loss law, the ratio of $\mathrm{E}_{X}$ quanta $\left(\mathrm{X}=\mathrm{K}, \mathrm{L}_{I I I}, \ldots\right)$ produced in the specimen and the standard can be written as follows:

$$
\frac{n_{A}}{n(A)}=C_{A} \frac{\bar{R}_{A}}{R(A)} \frac{\int_{E_{0}}^{E_{X}} \frac{\Psi_{X}^{A}(E)}{[d E / d \rho s]_{s p}} d E}{\int_{E_{o}}^{E_{X}} \frac{\Psi_{X}^{A}(E)}{[d E / d \rho s]_{s t}} d E}
$$

where $\mathrm{R}(\mathrm{A})$ is relative to pure $\mathrm{A}$ standard, $\overline{\mathrm{R}}_{A}$ to the specimen (for element A). $\mathrm{E}$ is the mean electron energy, $\Psi_{X}(\mathrm{E})$ the ionization cross section of the A atoms for the $\mathrm{X}$ level, $\mathrm{E}_{X}$ the ionization energy for this level. $\mathrm{dE} / \mathrm{d}(\rho \mathrm{s})$ is the retardation equation of the electrons in the target, $\rho$ its specific mass, $s$ the length of the path traversed by the electrons in the target. The subscripts st and $\mathrm{sp}$ are respectively related to standard and specimen. By using a well known theorem, this expression can be simplified to:

$$
\frac{\mathrm{n}_{\mathrm{A}}}{\mathrm{n}(\mathrm{A})}=\mathrm{C}_{\mathrm{A}} \frac{\overline{\mathrm{R}}_{\mathrm{A}}}{\mathrm{R}(\mathrm{A})} \frac{(\mathrm{dE} / \mathrm{d} \rho \mathrm{s})_{\mathrm{st}, \mathrm{E}},}{(\mathrm{dE} / \mathrm{d} \rho \mathrm{s})_{\mathrm{sp}, \mathrm{E},}}
$$

Since the ratio of stopping powers $\mathrm{s}=\mathrm{dE} / \mathrm{d} \rho \mathrm{s}$ is rather insensitive to the energy $\mathrm{E}$, the second factor on the righthand side may be calculated for some mean value of the energy, i.e., $E_{1}=\left(E_{0}+E_{X}\right) / 2$. Whence, with the usual notation:

$$
\left(\mathrm{n}_{A} / \mathrm{n}(\mathrm{A})\right)=\mathrm{C}_{A}\left(\overline{\mathrm{R}}_{A} / \mathrm{R}(\mathrm{A})\right)\left(\mathrm{s}(\mathrm{A}) / \overline{\mathrm{s}}_{A}\right)
$$

or

$$
\left(\mathrm{n}_{A} / \mathrm{n}(\mathrm{A})\right)=\left(\alpha_{A} \mathrm{C}_{A} / \bar{\alpha}\right)
$$

with

$$
\alpha_{A}=\mathrm{s}(\mathrm{A}) / \mathrm{R}(\mathrm{A}) \text { and } \bar{\alpha}=\overline{\mathrm{S}}_{A} / \overline{\mathrm{R}}_{A}
$$

The "second approximation" assumes that

$$
\bar{\alpha}=\Sigma \alpha_{j} \mathrm{C}_{j}
$$


This simplification can hardly be justified, but it works! Of course, the $\alpha$ 's depend on the incident energy $\mathrm{E}_{o}$. As used by Poole and Thomas, it has been very successful for the analysis of binary alloys or compounds.

For a more exact calculation, equation (7) is rewritten:

$$
\frac{n_{A}}{n(A)}=C_{A} \frac{\bar{R}_{A} / \bar{S}_{A}}{R(A) / S(A)}
$$

where

$$
S(A)=\int_{E_{0}}^{E_{X}^{A}} \frac{\Psi_{X}^{A}(E) d E}{[d E / d \rho s]_{A}}
$$

and

$$
\bar{S}_{\mathrm{A}}=\int_{\mathrm{E}_{0}}^{\mathrm{E}_{\mathrm{X}}^{\mathrm{A}}} \frac{\Psi_{\mathrm{X}}^{\mathrm{A}}(\mathrm{E}) \mathrm{dE}}{[\mathrm{dE} / \mathrm{d} \rho \mathrm{s}]_{\text {complex target }}}
$$

The $\mathrm{R}$ and $\mathrm{S}$ factors have to be calculated for the standard (pure element) and for the specimen (complex material).

As far as the $\mathrm{S}$ factor is concerned, an exact calculation can be carried out in. both cases by calculating equation (13a) and (13b) with the help of some analytical laws for $\Psi$ and $\mathrm{dE} / \mathrm{d} \rho$ s.

\section{CALCULATION OF S FACTOR ${ }^{1}$}

Let us consider a target containing elements $\mathrm{A}, \mathrm{B}, \mathrm{C}, \ldots$ of atomic masses $A_{i}, \ldots$ atomic numbers $Z_{i}, \ldots$ and mass concentrations $C_{i}, \ldots$ such that $\sum_{i} C_{i}=1$.

Let this target be bombarded with electrons of initial energy $\mathrm{E}_{o}=\mathrm{e} \cdot \mathrm{V}_{o} ; \mathrm{E}_{X}$ is the ionization energy of the $\mathrm{X}$ level of atoms $\mathrm{A}$. We shall study the element $A$.

${ }^{1}$ See Brit. J. Appl. Phys. (1968) ser. 2, vol. 1, 685. 
If $\mathrm{dn}_{X}$ is the mean number of ionizations of the $\mathrm{X}$ level of the $\mathrm{A}$ atoms produced by the electrons along the path increment ds:

$$
\begin{aligned}
& \mathrm{dn}_{\mathrm{X}}=\mathrm{C}_{\mathrm{A}} \rho \frac{\mathrm{N}}{\mathrm{A}} \Psi_{\mathrm{A}}^{\mathrm{X}}(\mathrm{E}) \mathrm{ds} \\
& \mathrm{n}_{\mathrm{X}}=\mathrm{C}_{\mathrm{A}} \cdot \frac{\mathrm{N}}{\mathrm{A}} \int_{\mathrm{E}_{0}}^{\mathrm{E}_{\mathrm{X}}} \frac{\Psi_{\mathrm{A}}^{\mathrm{X}}(\mathrm{E})}{\mathrm{dE} / \mathrm{d} \rho \mathrm{s}} \mathrm{dE}
\end{aligned}
$$

assuming that $(\mathrm{dE} / \mathrm{d} \rho \mathrm{s})$ is defined and is continuous along the path and that $\mathrm{dn}_{X}$ might be integrated. $\mathrm{N}$ is Avogadro's number. Bethe (1930) [3] and Bethe, Rose, and Smith (1938) [4] have given the following expressions for $\Psi$ and $\mathrm{dE} / \mathrm{d} \rho$ s:

a. Ionization Cross Section:

$$
\Psi_{A} \mathrm{x}(\mathrm{E})=\frac{\pi \mathrm{e}^{4}}{\mathrm{E} \cdot \mathrm{E}_{\mathrm{X}}} Z_{\mathrm{X}} \mathrm{b}_{\mathrm{X}} \log \frac{4 \mathrm{E}}{\mathrm{B}_{\mathrm{X}}}
$$

$\mathrm{Z}_{X}$ : number of electrons on $\mathrm{X}$ level

$\mathrm{b}_{X}: \mathrm{b}_{K}=0.35, \mathrm{~b}_{L}=0.25$

$\mathrm{B}_{X}=1.65 \mathrm{E}_{X}$ according to Bethe, but Worthington and Tomlin (1956) [20] gave a better form for low excitation energy as $\mathrm{B}_{X}=4 \mathrm{E}_{X}$. We shall take the value of $4 \mathrm{E}_{X}$ for electron probe microanalysis, where the electron energy is quite small $(<40 \mathrm{keV})$; we then have $\Psi \rightarrow 0$ when $\mathrm{E} \rightarrow \mathrm{E}_{X}$.

The $b_{X}$ value does not appear in the atomic number effect correction (see later).

If $\mathrm{U}=\frac{\mathrm{E}}{\mathrm{E}_{\mathrm{X}}}, \mathrm{U}_{\mathrm{o}}=\frac{\mathrm{E}_{\mathrm{o}}}{\mathrm{E}_{\mathrm{X}}}$ (over-voltage ratio)

$$
\Psi_{A}^{X}(U)=\pi e^{4} \frac{Z_{X} b_{X}}{E_{X}^{2}} \cdot \frac{1}{U} \cdot \log U
$$


b. Retardation Equation:

$$
\frac{\mathrm{dE}}{\mathrm{d} \rho \mathrm{s}}=-\frac{2 \pi \mathrm{e}^{4} \mathrm{~N}}{\mathrm{U} \cdot \mathrm{E}_{\mathrm{X}}} \sum_{\mathrm{i}} \mathrm{C}_{\mathrm{i}} \cdot \frac{\mathrm{Z}_{\mathrm{i}}}{\mathrm{A}_{\mathrm{i}}} \log \frac{1.166 \mathrm{UE} \mathrm{E}_{\mathrm{X}}^{\mathrm{A}}}{\mathrm{J}_{\mathrm{i}}}
$$

$\mathrm{J}_{i}$ : mean ionization potential for the $\mathrm{i}$ atoms.

These equations suppose that the electron energy is high enough i.e., $E>E_{X}^{A}$ in equation (15) and $U \cdot E_{X}^{A}>J_{i}$ for all the target atoms ir equation (17).

Let

$$
\sum_{i} C_{i} \frac{Z_{i}}{A_{i}}=M
$$

and

$$
]_{i}\left[\left(\frac{1.166 \mathrm{E}_{\mathrm{X}}^{\mathrm{A}}}{\mathrm{J}_{\mathrm{i}}}\right)^{\mathrm{C}_{i} z_{i} / \mathrm{A}_{i}}=\mathrm{W}^{\mathrm{M}}\right.
$$

or

$$
\begin{aligned}
\log W & =\frac{1}{M} \sum_{i} C_{i} \cdot \frac{Z_{i}}{A_{i}} \log \frac{1,166 E_{x}^{A}}{J_{i}} \\
& =\frac{1}{M} \sum_{i} C_{i} \frac{Z_{i}}{A_{i}} \log W_{i} x
\end{aligned}
$$

This leads to

$$
\frac{\mathrm{dE}}{\mathrm{d} \rho \mathrm{s}}=-\frac{2 \pi \mathrm{e}^{4} \mathrm{~N}}{\mathrm{UE} \mathrm{E}_{\mathrm{X}}} \cdot \mathrm{M} \log \mathrm{U} \cdot \mathrm{W}
$$

and from (14)

$$
n_{X}=C_{A} \frac{N}{A} \cdot \frac{Z_{X} b_{X}}{2 N} \int_{1}^{U_{0}} \frac{\log U}{M \log U \cdot W} \cdot d U
$$


Let $(1 / \mathrm{S})=\mathbf{n}_{X} \cdot(\mathrm{A} / \mathrm{N}) \cdot\left(1 / \mathrm{C}_{A}\right)$, where $(1 / \mathrm{S})$ is a function which can be used to represent the electron mass stopping effect in atomic number effect corrections.

Putting $X=U \cdot W$, we can integrate $(1 / S)$ in terms of logarithmic integral (li) function and we get:

$$
\begin{aligned}
\frac{1}{S}= & \frac{Z_{X} b_{X}}{2 N} \cdot \frac{1}{M}\left\{\left(U_{0}-1\right)-\frac{1}{W} \log W \times\right. \\
& \left.\times\left[1 i\left(U_{0} . W\right)-1 i(W)\right]\right\}
\end{aligned}
$$

The above equation allows the calculation of $(1 / \mathrm{S})$ in any target.

In a target made of a pure element, it reduces to an already known equation (Archard, Mulvey, 1962) [1].

By assumption, we must have $\mathrm{U}_{0} \cdot \mathrm{W}>1$. If $\mathrm{W} \rightarrow 1,(1 / \mathrm{S})$ remains finite as can be seen in the series expansion:

$$
\operatorname{li} x=C+\log |\log x|+\sum_{s=1}^{s=\infty} \frac{(\log x)^{8}}{\text { s. s! }} .
$$

$\mathrm{C}=$ Euler's constant. The above expression allows the li function to be easily computed.

\section{A Practical Way of Calculating (1/S) for a Target of Known Composition}

The terms $W_{i}^{\mathrm{X}}=\left(1.166 \mathrm{E}_{X}^{\mathrm{A}} / \mathrm{J}_{i}\right)$ can first be calculated. We have tabulated $W_{i}^{X}$ for all elements of $3 \leqslant Z \leqslant 92$, taking the ionization energy $\mathrm{E}_{X}$ for the $\mathrm{K}$ level and $\mathrm{L}$ level from the tables of Bearden (1964) [2] and Blochin (1957) [6] (Fig. 1). We have used $\mathbf{J}_{i=11.5 Z_{i}}$ which might not be the best form for $\mathrm{J}$ ( $\mathrm{Ex}$ and $\mathrm{Ji}$ in $\mathrm{eV}$ ).

Afterwards one can calculate M (Eq. 18) and log W (Eq. 20).

The assumptions $\mathrm{U}_{o} \cdot \mathrm{W}_{i}^{\mathrm{X}}>1$ involve a theoretical minimum value for $\mathrm{U}_{\boldsymbol{\theta}}$, while in practical cases its value will always be greater. As a matter of fact, a small value of $W_{i}^{X}$ is found for analysis of a light element in a heavy matrix, hence a low $\mathrm{E}_{X}$ and a high $\mathrm{U}_{0}$.

One can then calculate (1/S). Practically, $\left(Z_{X} \cdot b_{X} / 2 N\right)$ need not be calculated as this term vanishes in the correction coefficient. One then calculates a term $\left(1 / S^{\prime}\right)$.

We have tabulated

$$
\left(\mathrm{M} / \mathrm{S}^{\prime}\right)=\mathrm{U}_{o}-1-(1 / \mathrm{W}) \log \mathrm{W}\left(\mathrm{li} \mathrm{U}_{o} \cdot \mathrm{W}-\mathrm{li} \mathrm{W}\right)
$$




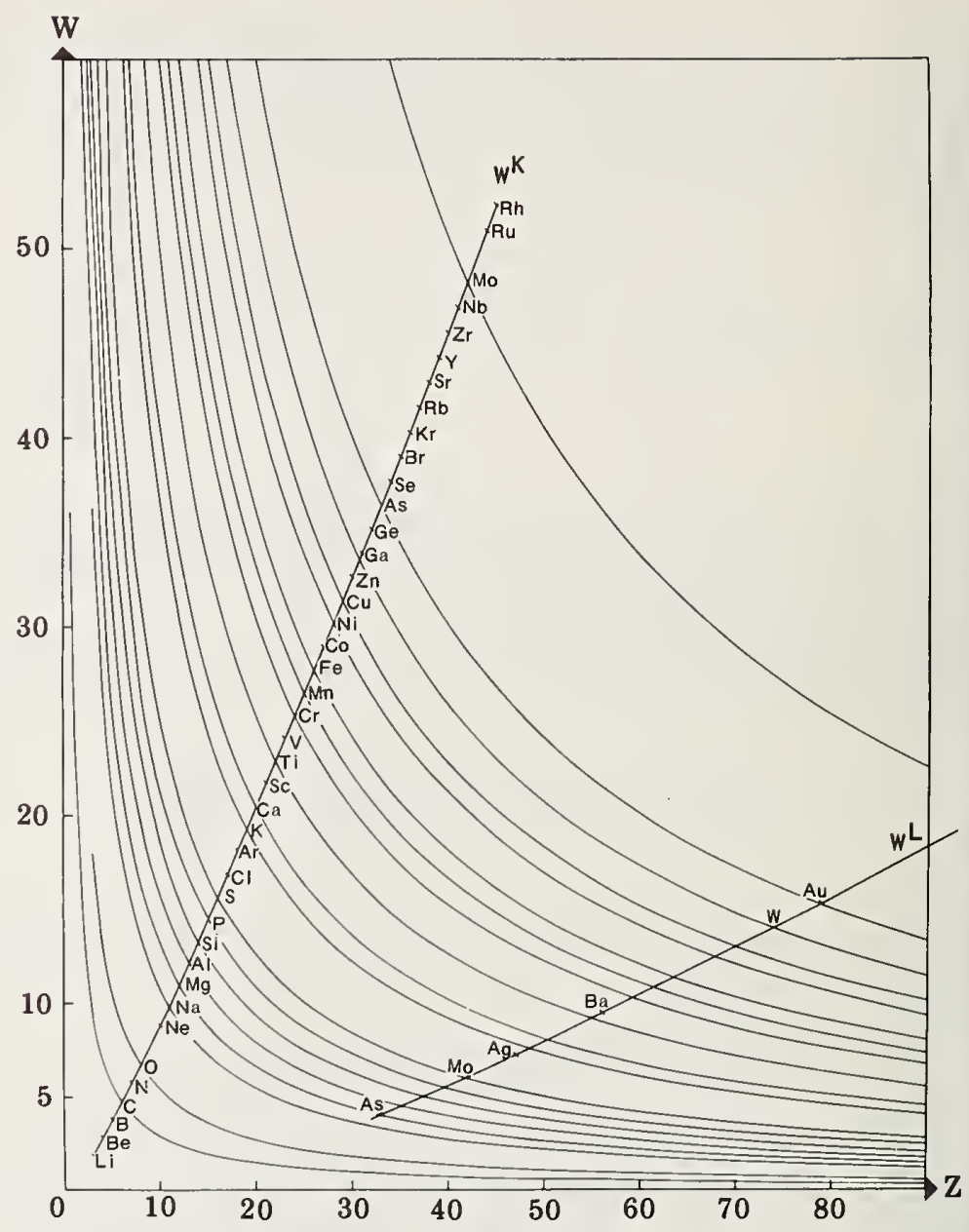

Figure 1. $\mathrm{W}_{i}^{X}=\left(1.166 \mathrm{E}_{X} / 11.5 \mathrm{Z}_{i}\right)$.

for different values of $\mathrm{W}$ and $\mathrm{U}_{o}$ (Figs. $\left.2,3,4\right)$ as the table of $\left(\mathbf{M} / \mathrm{S}^{\prime}\right)$ values can be used for any target.

\section{Applications}

The "universal" curves (Figs. $2,3,4)$ give $\left(\mathrm{M} / \mathrm{S}^{\prime}\right)$ from $\mathrm{U}_{o}$ and $\mathrm{W}$ values. W for an alloy can be calculated from $W_{A}^{\mathrm{X}}$ in pure specimens (Fig. 1), for the element analyzed, according to formula (20).

For instance, in copper-gold alloys where copper $\left(\mathbf{C u ~} \mathbf{K}_{\alpha}\right)$ is analyzed, and in copper-aluminium alloys where aluminium $\left(\mathrm{Al} \mathrm{K}_{\alpha}\right)$ is analyzed, W variations have been plotted against copper 


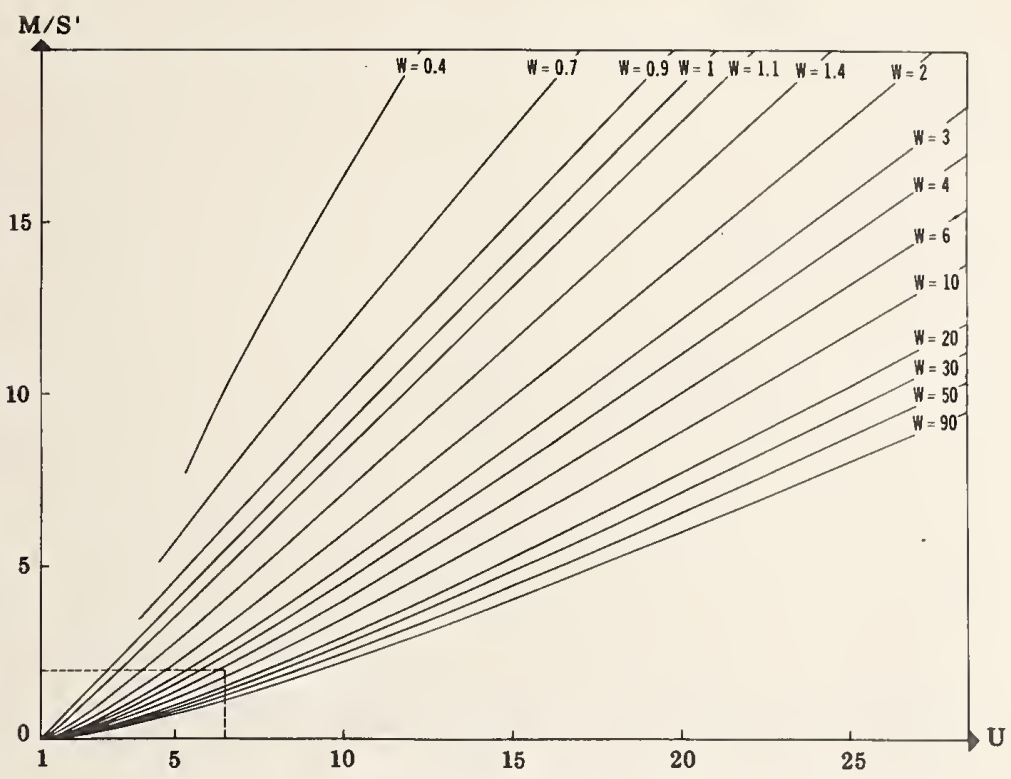

Figure 2. $M / S^{\prime}$ versus $U$ for different $W$ values, $1<U<30$.

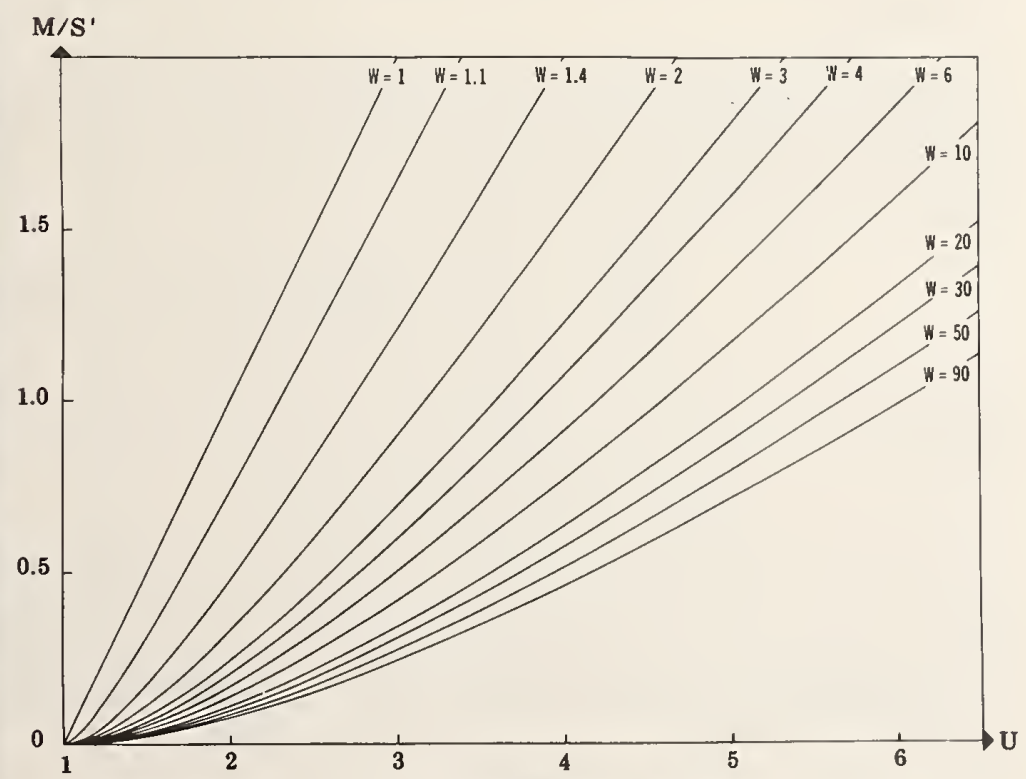

Figure 3. $M / S^{\prime}$ versus $U$ for different $W$ values, $I<U<6$. 


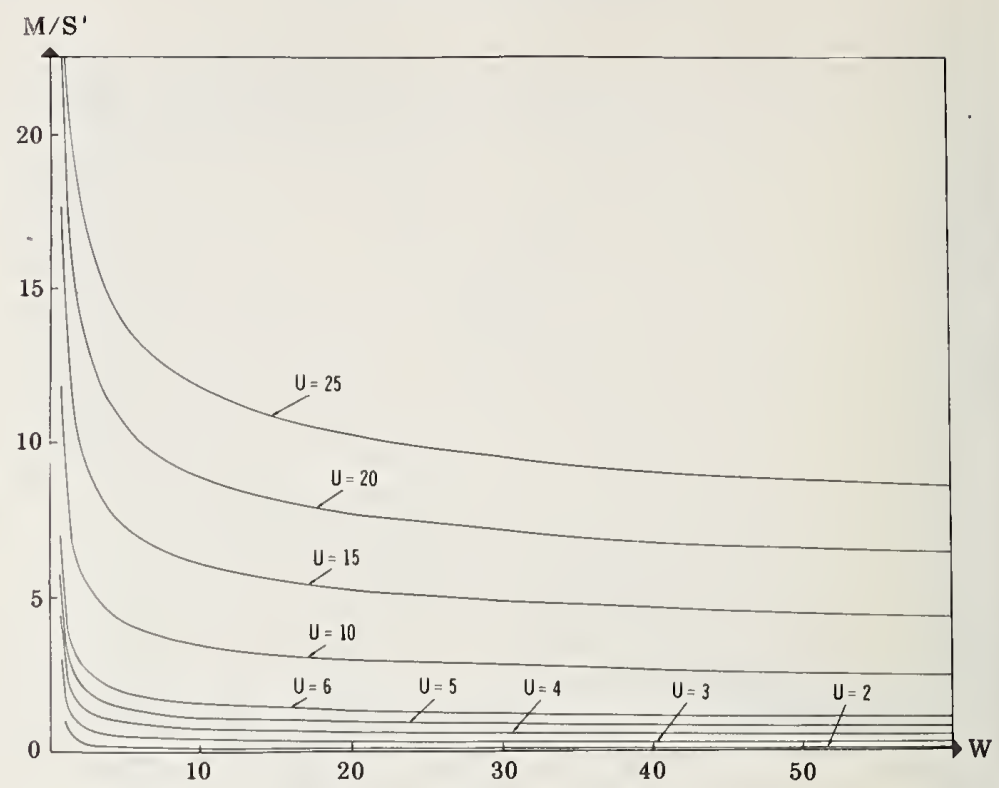

Figure 4. M/S' versus $W$ for different $U$ values.

concentration (Fig. 5). Generally, W is not a linear function of composition.

In a copper-gold alloy, using values for the backscattering coefficient R computed by Duncumb (1966) [14] from Bishop's measurements (Fig. 6) and (1/S') values from equation (23), we have calculated the correction coefficients

$$
\frac{\mathrm{k}}{\mathrm{C}}=\left[\frac{\mathrm{R}}{\mathrm{S}}\right] \text { sample } /\left[\frac{\mathrm{R}}{\mathrm{S}}\right] \text { standard }
$$

for different accelerating voltages and against concentrations (Fig. 7).

\section{Comparison with Other Results}

P. M. Thomas (1964) [18] supposes that one may substitute an average value for the stopping power $(\mathrm{dE} / \mathrm{d} \rho \mathrm{z})$ in the range $\mathrm{E}_{o}$ to $\mathrm{E}_{X}$ and takes this quantity outside the integral (14). Writing $\mathrm{s}=(\overline{\mathrm{dE} / \mathrm{d} \rho \mathrm{z}})$ (different from our $\mathrm{S}$ ) she assumes that for a specimen consisting of $n$ elements in concentrations $\mathrm{C}_{i}$

$$
\left(\frac{s}{R}\right)=\sum_{i} C_{i}\left(\frac{s}{R}\right)_{i}
$$




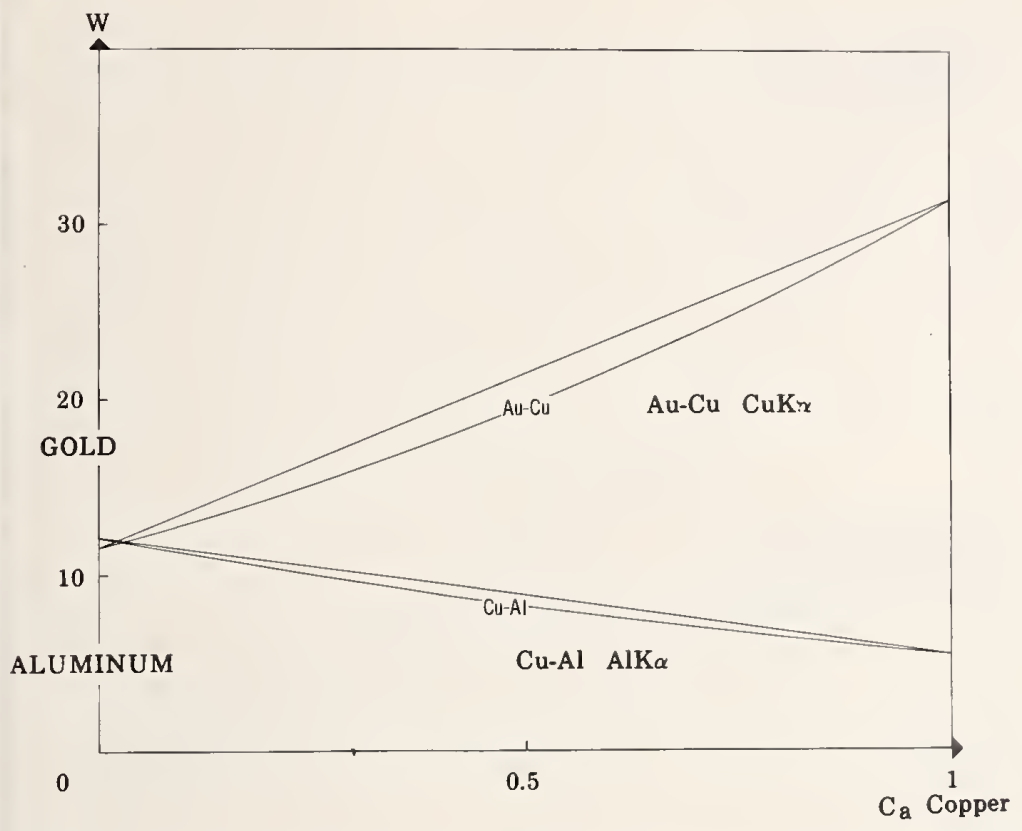

Figure 5. W versus copper concentration in copper-gold alloy (copper analyzed) and in copper-aluminium alloy (aluminium analyzed). A linear approximation has also been traced.

$\mathrm{R}$ is calculated from Brandt's data on the energy distribution of electrons backscattered from pure element targets. The values of $\mathrm{s}$ she used are taken from the tabulations by Nelms $(1956,1958)$ [16] based on the Bethe formula for electron stopping power. The value of s chosen for estimating the correction is that corresponding to an energy half way between $\mathrm{E}_{o}$ and $\mathrm{E}_{X}$.

Though Nelms' tables are limited to $10 \mathrm{keV}$ and are not very practical for use in micro-probe analysis conditions, Thomas' results are interesting to compare with ours since Nelms used for her tables an adjustment for the $\mathbf{J}$ variation law, and discussed the validity of calculations with the Bethe formulae. If we consider a 50-50 copperaluminium alloy in which aluminium is analyzed with $\mathrm{E}_{o}=10 \mathrm{keV}$, we find with our method $(1 / S)_{\text {spec. }} /(1 / S)_{\text {stand. }}=1.144$ and with Thomas's method 1.137. The discrepancy is not important in this case. Figure 7 might also be compared with the same computed by Archard and Mulvey. It should also be interesting to compare the $\mathrm{k} / \mathrm{c}$ values obtained here with some others deducted from direct 


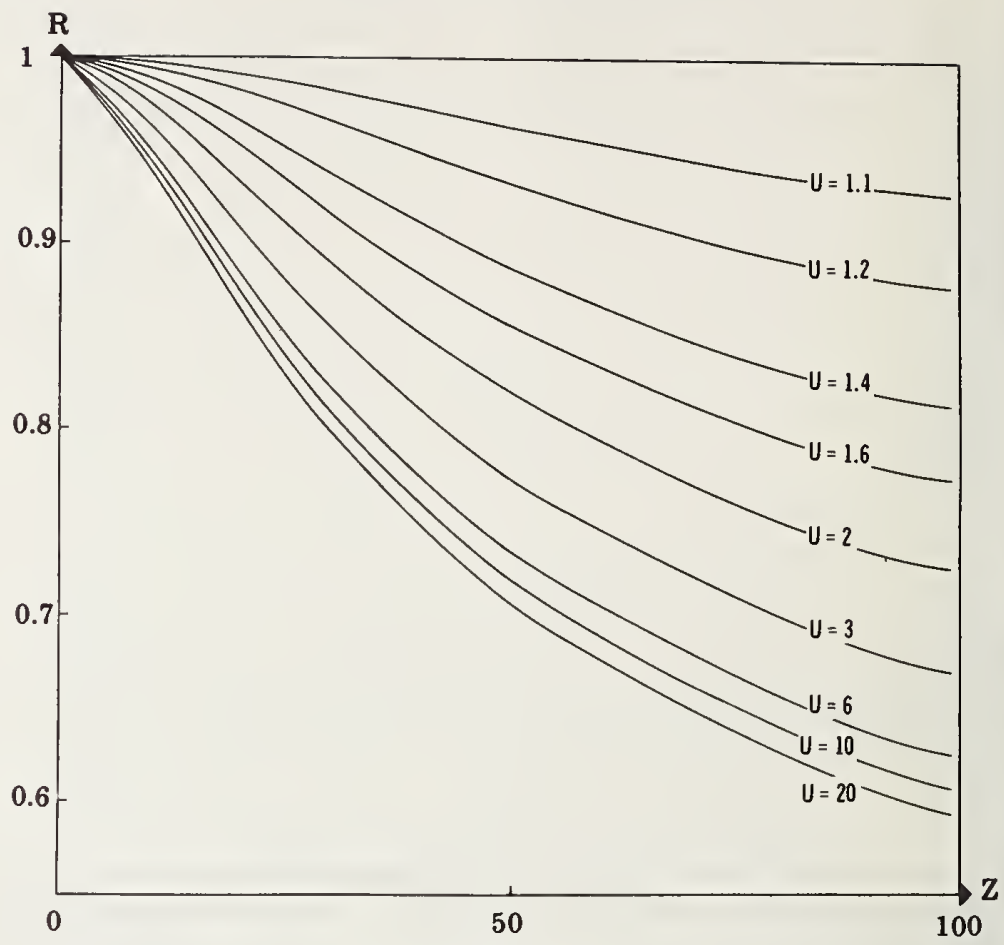

Figure 6. $\mathrm{R}$ versus $\mathrm{Z}$ for different $\mathrm{U}$ values according to Duncumb.

calculation of x-ray production by Monte Carlo methods according to Green (1962) [15] and Bishop (1965) [5].

The analytical forms show that approximation using a "mean atomic number" in order to have the same calculation with a mixed target as in pure elements is generally physically unfounded and can lead to important relative errors.

The validity of the present calculation is limited, especially in cases of small over-voltage: by approximation inherent in the Bethe formulae; by the values of the coefficients we used; and particularly because we neglect straggling by taking the mean electron energy as parameter (cf. Cosslett, 1964 [9], 1965 [10] and Brown, 1965 [7]). Since the atomic number effect is more important when the overvoltage is low the present model would need to be refined, using for instance the Walske (1952 and 1956) [19] calculations for extending the validity of Bethe's formula, or adjusting coefficient and parameter values with experimental measurement (Duncumb and da Casa). 


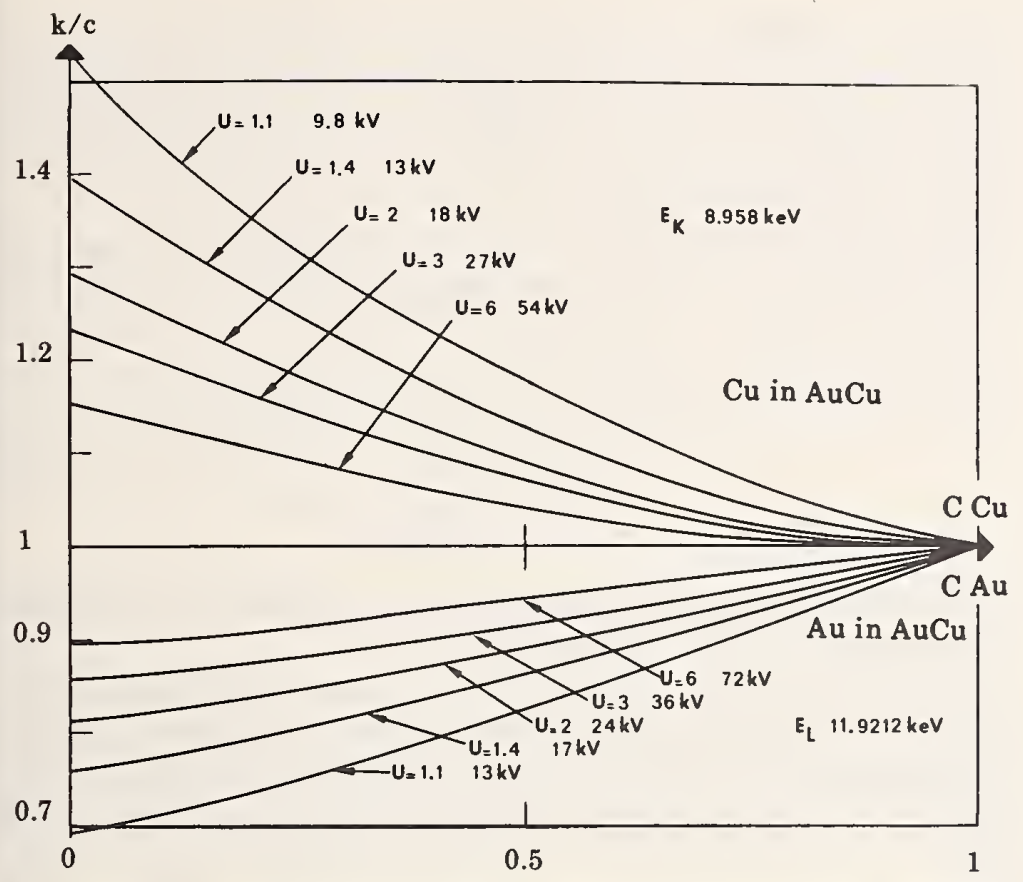

Figure 7. The atomic number effect correction factor versus concentration.

\section{E. CALCULATION OF THE R FACTORS}

The $\mathrm{R}$ factors can be computed for pure elements by using analytical expressions for $\Psi$ and $\mathrm{dE} / \mathrm{d} \rho$ s, provided that the energy distribution of electrons backscattered from these pure targets is known.

$$
1-\mathrm{R}(\mathrm{A})=\frac{\int_{\mathrm{E}_{\mathrm{o}}}^{\mathrm{E}_{\mathrm{X}}^{\mathrm{A}}} \eta(\mathrm{E}) \frac{\Psi_{\mathrm{X}}^{\mathrm{A}}(\mathrm{E})}{\mathrm{dE} / \mathrm{d} \rho \mathrm{S}} \mathrm{dE}}{\int_{\mathrm{E}_{\mathrm{o}}}^{\mathrm{E}_{\mathrm{X}}^{\mathrm{A}}} \frac{\Psi_{\mathrm{X}}^{\mathrm{A}}(\mathrm{E})}{\mathrm{dE} / \mathrm{d} \rho \mathrm{S}} \mathrm{dE}}
$$

with

$$
\eta(\mathrm{E})=\int_{\mathrm{E}}^{\mathrm{E}_{\mathrm{o}}} \frac{\mathrm{d} \eta}{\mathrm{dE}} d \mathrm{E}
$$


where the $\eta(E)$ and $d E / d \rho S$ apply to the complex or the pure target.

The analytical expressions for $\mathrm{d} \eta / \mathrm{dE}$ or $\eta(E)$ are not available. The integrations have to be carried out using either experimental data for $\eta(e)$ or calculated data (e.g., by Monte Carlo method or by transport equation method).

An alternative way would be to start from the $\phi(\rho \mathrm{z})$ functions determined either experimentally or theoretically.

$$
R=K \frac{\left[\int_{0}^{\infty} \phi(\rho \mathrm{z}) \mathrm{d} \rho \mathrm{z}\right]}{\int_{\mathrm{E}_{\mathrm{o}}}^{\mathrm{E}_{\mathrm{X}}} \frac{\Psi_{\mathrm{X}}(\mathrm{E})}{\mathrm{dE} / \mathrm{d} \rho \mathrm{S}} \mathrm{dE}}
$$

where $\mathrm{K}$ is a normalizing factor introduced to express the numerator and denominator in the same units.

Direct measurements of the $\mathrm{R}$ factor are also possible as shown by Dérian [13], who has designed a special type of target such that all the electrons lose all their energy inside the target material. The $\mathrm{R}$ factor is readily calculated by comparison with a normal plane target.

Dérian applied this method to the determination of $\mathrm{R}$ in pure targets of $\mathrm{Al}, \mathrm{Cu}$, and $\mathrm{Ag}$ for a series of over-voltage ratios $\mathrm{U}_{o}=\mathrm{E}_{o} / \mathrm{E}_{k}$. As these values are in good agreement with Duncumb's evaluations (based on equation (27) and $\eta(E)$ derived from Bishop experimental and theoretical results) one may be confident in the latter. Other calculations of the R factor (by Green, from older experimental data, by Brown from its T.E.P. calculation) seem to give figures which are too low.

We are finally faced with the calculation of $\overline{\mathrm{R}}_{A}$, i.e., the $\mathrm{R}$ factor for A element in a complex target. This seems a priori to be a formidable task, as this factor like the $\mathrm{S}$ factor, must be dependent on $\mathrm{U}_{o}$, on all the $\mathrm{Z}_{i}, \mathrm{C}_{i}$, and $\mathrm{E}_{X}^{\mathrm{A}}$, or equivalently on $\mathrm{U}_{o}$, all the $\mathrm{Z}_{i}, \mathrm{C}_{i}$, and $\mathrm{E}_{o}\left(=\mathrm{E}_{X}^{\mathrm{A}} \mathrm{U}_{o}\right)$.

Let us look again at the calculation of the $S$ factor. The case of $A$ radiation in pure $A$ is very simple ( $E_{X}^{A}$ is a $Z_{A}$ function). $S(A)$ depends only on $\mathrm{U}_{o}$ and $\mathrm{Z}_{A}$. But the case of $A$ radiation emitted from an otherwise pure B target is more complicated. Three variables have to be considered: $\mathrm{U}_{o}^{\mathrm{A}}=\mathrm{E}_{o} / \mathrm{E}_{X}^{\mathrm{A}}, \mathrm{E}_{X}^{\mathrm{A}}, \mathrm{Z}_{B}$. In other words, the $\mathrm{S}$ factor depends on $\mathrm{W}$ and $\mathrm{U}_{o}^{\mathrm{A}}=\mathrm{E}_{o} / \mathrm{E}_{X}^{\mathrm{A}}$ but $\mathrm{W}$ depends on $\mathrm{Z}_{A}$ and $\mathrm{Z}_{B}$.

For a complex target, we have to calculate a mean $\bar{W}$ depending on the $Z_{i}$ of the components, but a simple relation like $\bar{S}_{A}=\sum_{i} C_{i} S_{i}$ does not hold, and is even meaningless. 
The situation does not appear to be better for the $\mathrm{R}_{A}$ factor. In a pure $\mathrm{B}$ target, $\mathrm{R}$ must evidently depend on $\mathrm{U}_{o}^{\mathrm{A}}=\mathrm{E}_{o} / \mathrm{E}_{X}^{\mathrm{A}}, \mathrm{Z}_{A}$ and $\mathrm{Z}_{B}$.

According to Bishop (1966) [5], a valid approximation assumes that $\mathrm{R}_{A}$ depends only on $\mathrm{U}_{o}^{\mathrm{A}}=\mathrm{E}_{o} / \mathrm{E}_{X}^{\mathrm{A}}$ and $\mathrm{Z}_{B}$, i.e., the excitation ratio for the analyzed element and the atomic number of the target, regardless the $Z$ of the analyzed element, or more exactly, regardless of its excitation energy. The quantity to be considered is $\mathrm{U}_{o}^{\mathrm{A}}$, which in fact combines two parameters: primary energy $\mathrm{E}_{o}$, atomic number $Z_{A}$ of the analyzed element (or $E_{X}^{A}$ equivalently). Once this assumption is allowed, calculation for a composite target is possible, as follows:

$$
\overline{\mathrm{R}}_{A}=\sum_{i} \mathrm{C}_{i} \mathrm{R}_{i}
$$

where $\mathrm{R}_{i}$ is calculated for the excitation ratio $\mathrm{U}_{\theta}^{\mathrm{A}}=\mathrm{E}_{o} / \mathrm{E}_{X}^{\mathrm{A}}$ in the pure i targets.

The simplification has been shown to be a fairly good one by Bishop, who compared the results of such calculations with more direct ones carried out with the help of a Monte Carlo computation. But, there is, a priori, no reason for such a comparison to be more valid for the R's than for the S's.

It would be interesting to look at the possibility of $\mathrm{W}$ being a more reliable parameter, because $\mathrm{W}$ is determined by the properties of the target material and of the excited $\mathrm{A}$ atoms. We should then need tables of $\mathrm{R}_{A}$ versus $\mathrm{W}$ and $\mathrm{U}_{o}^{\mathrm{A}}=\mathrm{E}_{o} / \mathrm{E}_{X}^{\mathrm{A}}$. This suggestion seems reasonable, since $W$ depends essentially on the target properties, i.e., in the case of a pure B target (A emission in otherwise pure B) $Z_{B}, J_{B}$, and $E_{X}^{A}$ (i.e., $Z_{A}$ ), all quantities on which the backscattered electron energy and number might be dependent.

\section{F. THIN FILMS AND EXTRACTION REPLICAS}

If we want the relative concentrations of two elements, $\mathrm{A}$ and $\mathrm{B}$, in a thin target, i.e., a target whose thickness is very much less than the range in terms of beam energy (for instance an extraction replica or a thin foil for electron microscopy), the atomic number effect takes a different form.

For such an analysis, we use a probe with constant intensity and energy on the same point of the target to measure the apparent concentration $\mathrm{K}_{A}=\left(\mathrm{J}_{A} / \mathrm{J}(\mathrm{A})\right)$ of $\mathrm{A}$ and that of $\mathrm{B}$, and we generally write the result as the ratio $\mathrm{K}_{A} / \mathrm{K}_{B}$. The standards are supposed to be the massive pure elements as usual.

If we assume that the effect of backscattering in the specimen can be neglected in this case (since we are calculating a ratio) and that 
the effects of fluorescence and absorption can be neglected in the specimen and corrected in the standard, we have with the same symbols as above:

in the specimen

$$
\mathrm{dn}_{\mathrm{x}}=\mathrm{C}_{\mathrm{A}} \frac{\mathrm{N}}{\mathrm{A}} \quad \Psi_{\mathrm{A}}^{\mathrm{X}}(\mathrm{E}) \mathrm{d} \rho \mathrm{s}
$$

in the massive standard,

$$
n_{X}=\frac{N}{A} \int_{E_{0}}^{E_{X}^{A}} \frac{\Psi_{A}^{X}(E)}{d E / d \rho s} d E
$$

and with the analytical expressions for $\Psi$ and $\mathrm{dE} / \mathrm{d} \rho \mathrm{s}$ :

$$
\begin{aligned}
& \mathrm{dn}_{\mathrm{X}}=\mathrm{C}_{\mathrm{A}} \frac{\mathrm{N}}{\mathrm{A}} \cdot \frac{\pi \mathrm{e}^{4}}{\mathrm{E}_{\mathrm{o}} \mathrm{E}_{\mathrm{X}}^{\mathrm{A}}} \cdot \mathrm{Z}_{\mathrm{X}} \mathrm{b}_{\mathrm{X}} \log \mathrm{U}_{\mathrm{o}}^{\mathrm{A}} \mathrm{d} \rho \mathrm{s} \\
& \mathrm{n}_{\mathrm{X}}=\frac{\mathrm{N}}{\mathrm{A}} \cdot \frac{\mathrm{Z}_{\mathrm{X}} \mathrm{b}_{\mathrm{X}}}{2 \mathrm{~N}} \int_{1}^{\mathrm{U}_{0}^{\mathrm{A}}} \frac{\log \mathrm{U}}{\mathrm{M}_{\mathrm{A}} \log \mathrm{U} \cdot \mathrm{W}_{\mathrm{A}}^{\mathrm{X}}} \cdot \mathrm{dU}
\end{aligned}
$$

with

$$
\mathrm{U}_{\mathrm{o}}^{\mathrm{A}}=\frac{\mathrm{E}_{\mathrm{o}}}{\mathrm{E}_{\mathrm{X}}^{\mathrm{A}}}, \mathrm{M}_{\mathrm{A}}=\frac{\mathrm{Z}_{\mathrm{A}}}{\mathrm{A}_{\mathrm{A}}}, \mathrm{W}_{\mathrm{A}}^{\mathrm{X}}=\frac{1.166 \mathrm{E}_{\mathrm{X}}^{\mathrm{A}}}{\mathrm{J}_{\mathrm{A}}}
$$

$\mathbf{R}_{A}$ is the backscattering factor of standard $\mathrm{A}$ for the over-voltage ratio $\mathrm{U}_{0}^{\mathrm{A}}$, and,

$$
\frac{1}{\mathrm{~S}_{\mathrm{A}}}=\int_{1}^{\mathrm{U}_{0}^{\mathrm{A}}} \frac{\log \mathrm{U}}{\mathrm{M}_{\mathrm{A}} \cdot \log U \mathrm{~W}_{\mathrm{A}}^{\mathrm{X}}} \cdot \mathrm{dU}
$$

We can write as a first approximation:

$$
\mathrm{k}_{\mathrm{A}}=\frac{\mathrm{dn}_{\mathrm{X}}}{\mathrm{n}_{\mathrm{X}}}=\mathrm{C}_{\mathrm{A}} \cdot \frac{\pi \mathrm{e}^{4} 2 \mathrm{~N}}{\mathrm{E}_{\mathrm{o}}} \cdot \frac{\log \mathrm{U}_{\mathrm{o}}^{\mathrm{A}}}{\mathrm{E}_{\mathrm{X}}^{\mathrm{A}}} \cdot \frac{\mathrm{d} \rho \mathrm{s}}{\mathrm{R}_{\mathrm{A}} / \mathrm{S}_{\mathrm{A}}^{\prime}}
$$


If we put

$$
\begin{aligned}
& \mathrm{P}_{\mathrm{A}}=\frac{\log \mathrm{U}_{\mathrm{o}}^{\mathrm{A}}}{\mathrm{E}_{\mathrm{X}}^{\mathrm{A}} \mathrm{R}_{\mathrm{A}} / \mathrm{S}_{\mathrm{A}}^{\prime}} \\
& \frac{\mathrm{K}_{\mathrm{A}}}{\mathrm{K}_{\mathrm{B}}}=\frac{\mathrm{C}_{\mathrm{A}}}{\mathrm{C}_{\mathrm{B}}} \cdot \frac{\mathrm{P}_{\mathrm{A}}}{\mathrm{P}_{\mathrm{B}}}
\end{aligned}
$$

If $\mathrm{P}_{A}>\mathrm{P}_{B}$, the measured concentration in $\mathrm{A}$ against that of $\mathrm{B}$ is greater than the real concentration.

Example:

a. Analysis of very thin precipitates of $\mathrm{Ni}+\mathrm{Al}$ in a carbon replica $\left(P_{A l} / P_{N i}\right)=0.63$ at $30 \mathrm{keV}, 0.55$ at $20 \mathrm{keV}$.

b. Analysis of low copper concentrations in a thin foil of an aluminium-copper alloy $\left(\mathbf{P}_{A l} / \mathbf{P}_{C u}\right)=0.67$ at $30 \mathrm{keV}$.

In both cases the measured value of the aluminium concentration was less than the true value. The effect is just the opposite of what would be observed in a bulk target, and it is far greater.

If the specimen is not thin enough, or if the voltage is not sufficiently high, the effect must lie between those characteristic of a bulk target and of a thin foil; however, it seems difficult to calculate the correction or even to determine whether the factor is greater or less than one, and the absorption effect in the specimen is no more negligible.

Experimental Results:

$\mathrm{Ni}$ and $\mathrm{Al}$ containing precipitates were extracted from a semimartensitic stainless steel. Experimental results are given in the following table:

$$
\mathrm{N}_{\mathrm{i}} / \mathrm{A}_{1}\left(\exp -\mathrm{e}^{\text {al }}\right) \quad \mathrm{N}_{i} / \mathrm{A}_{1} \text { (corrected) }
$$

$\begin{array}{lll}30 \mathrm{kV} & 3.27 & 2.1\end{array}$

$\begin{array}{lll}20 \mathrm{kV} & 4.25 & 2.35\end{array}$

Without corrections, experimental results would indicate $\mathrm{Ni}_{2} \mathrm{Al}$ composition. After correction for atomic number effect, the results are in favor of $\mathrm{Ni} \mathrm{Al}$. This determination has been confirmed by electron diffraction $\left(\mathrm{Ni}_{3} \mathrm{Al}\right.$ was a possible precipitate). 
This thin film correction has also been studied by Duncumb from both theoretical and experimental viewpoints.

\section{Conclusion}

As it has been tentatively shown, the expressions used for the correction calculations depend on a lot of drastic assumptions and on the validity of some physical laws involved (ionization crosssections, continuous retardation law). The validity of the expressions used for these laws is known to be not very good, and probably limited to some more or less wide range of atomic numbers. Moreover, the validity of Bethe retardation law is generally questionable since it neglects energy straggling. This last effect probably modifies appreciably the energy loss distribution as a function of depth and, consequently, the ionization distribution with depth (the so-called $\phi(\rho z)$ function).

These limitations affect to the same extent the other approaches which would seem more accurate: Monte Carlo computation, resolution of Boltzmann transport equation. These approaches suffer basically from the same inaccuracies; moreover, they give numerical solutions and no analytical expressions for the $\mathrm{Z}$ and $\mathrm{A}$ corrections. One has perhaps to get rid of the feeling that analytical expressions are necessary and to convince oneself of the convenience of working with tables, the results of numerical methods being collected in these tables. Probably these numerical methods will give more and more accurate results through more realistic models of the electron behavior in massive targets. For everyday calculations, however, analytical expressions which would need only a limited quantity of input data seem to be desirable. It is hoped that such better expressions will be available in the future; in combination with tables they will permit corrections to be readily carried out in most of the common cases.

In conclusion, the need for more reliable data concerning the basic parameters $\left(\mu / \rho, \mathbf{W}_{K, L}, \mathrm{~J}, \ldots\right)$ as well as for the physical law involved $(\Psi, \mathrm{d} E / \mathrm{d} \rho \mathrm{s}$, multiple scattering, backscattering), must be stressed.

Experiments on composite targets (measurement of backscattered electron energy distribution, direct measurement of $\mathrm{R}$ factor, etc...) will enable a better check of the suggested computation of the $Z$ effect to be made. The case of non-metallic specimens, such as complex oxides, would perhaps deserve specially careful 
measurements. Finally, theoretical computations, based on the Monte Carlo model, the resolution of Boltzmann transport equation, the invariant imbedding method (Dashen [12]) or the older "collective" model, could perhaps be carried out with more refined basic laws. The case of thin films and extractions replica also requires careful attention.

\section{References}

[1] Archard, G. D., Mulvey, T., X-Ray Optics and X-Ray Microanalysis, Academic Press, New York, Stanford pp. 393-410 (1962).

[2] Bearden, J. A., US AEC, NYO 10586, (1964).

[3] Bethe, H. A., Ann. Phys. Lpz. 5, pp. 325-400 (1930).

[4] Bethe, H. A., Rose, M. E., Smith, L. P., Proc. Am. Phil. Soc. 78, pp. 573-585 (1938).

[5] Bishop, H. E., Proc. Phys. Soc. 18, pp. 855-866 (1965), and Thesis, Cambridge (1966).

[6] Blochin, M. A., V.E.B. Verlag Technik Berlin, pp. 422-426 (1957).

[7] Brown, D. B., Thesis, MIT (1965); see also: Ogilvie, R. E., Brown, D. B., Optique des Rayons X et Microanalyse, Hermann, Paris p. 139 (1967).

[8] Castaing, R., Advances in Electronics and Electron Physics 13, pp. 317-386 (1960).

[9] Cosslett, V. E., Brit. J. Appl. Phys. 15, pp. 107-109 (1964).

[10] Cosslett, V. E., Optique des Rayons X et Microanalyse, Orsay 1965, Hermann, Paris pp. 85-96 (1967).

[11] Cosslett, V. E., Thomas, R. N., Brit. J. Appl. Phys. 15, pp. 883-907, 12831300; 16, pp. 779-796 (1964).

[12] Dashen, R. F., Phys. Rev. 134, A 1025 -A 1032 (1964).

[13] Dérian, J. C., Rapport CEA R3052 (1966).

[14] Duncumb, P., Private Communication; see also the paper by Duncumb and Reed in this book.

[15] Green, M., Thesis, University of Cambridge (1962).

[16] Nelms, A. T., NBS, 577 (1956), Supplement (1958).

[17] Reed, S. J. B., Brit. J. Appl. Phys. 16, pp. 913-926 (1965).

[18] Thomas, P. M., AERE, R4593 (1964).

[19] Walske, M. C., Phys. Rev. 88, pp. 1283-1289 (1952) and Phys. Rev. 101, pp. 940-944 (1956).

[20] Worthington, C. R., Tomlin, S. G., Proc. Phys. Soc. 59, pp. 401-412 (1956). 



\title{
THE THEORY OF QUANTITATIVE ELECTRON PROBE MICROANALYSIS
}

\author{
I. B. BOROVSKII AND V. I. RYDNIK \\ Institute of Metallurgy, USSR Academy of Sciences, Moscow
}

\begin{abstract}
The authors propose a theoretical basis for a method to correct the results of electron probe microanalysis using an external elemental standard. The paper is divided into two parts, the first of which provides a general expression for the intensity of characteristic $\mathrm{x}$-radiation produced by the impact of electrons upon a thick anode. The following approximations were made in the quantitative calculation:
\end{abstract}

1. In order to calculate the effective range of ionization by electrons, in a given layer of anode material, the electron current through this layer is divided into two parts: the forward current (integrating over the scattering angles $0 \leqslant \phi_{\eta} \leqslant \pi / 2$ ) and the reverse current (integrating over the scattering angles $\pi / 2 \leqslant \phi_{b} \leqslant \pi$ ). Each of these currents is characterized by its energy distribution and by a mean cosine of angular distribution.

2. The energy distribution of the electrons in the anode is expressed in the form proposed by Makhov, and the values of the parameters $\underline{\mathbf{n}}$ and $\underline{\mathrm{p}}$ found experimentally by Dupouy et al.

3. The differential effective ionization cross-section of the given atomic level is taken in the form proposed by Cosslett and Green.

The expression for the ionization function by electron impact obtained under these assumptions can be evaluated by numerical integration, and it agrees satisfactorily with the experimental results of Castaing and Descamps.

However, the expression obtained for the $\mathrm{x}$-ray intensity cannot be presented in an analytic form in the general case. Therefore, the authors propose in the second part of the paper an approximation for the ionization function which allows a reduction of the numerical calculation. In this approximation, the two variable parameters, $n$ and $p$, are considered to be constant for a mean value chosen for one of them (n). The applicability of this approximation is tested by comparing with experimental results the variation of the intensity of characteristic $\mathrm{x}$-ray emission as a function of the overvoltage with 
respect to minimum excitation potential. A similar comparison was made between calculation and measurement of the $\mathrm{x}$-ray absorption as a function of the $\mathrm{x}$-ray emergence angle with respect to the target surface. Finally, we have derived expressions for the atomic number and absorption corrections. A slight modification of the fluorescence correction procedure of Castaing is also proposed. Results of comparing measured and calculated concentrations for a series of binary systems of elements are presented.

In local x-ray spectral analysis with the aid of fine electron beams, the method of internal standards is not applicable, and the use of many-element standards in the method of external standards is complicated by the difficulty in preparing highly homogeneous specimens. Therefore, in microprobe analysis one chiefly uses a method of external standards, in which the standards are prepared from pure elements.

It was shown by Castaing (1951) [1] that in the first' approximation the weight concentration $C_{i}$ of the $i^{\text {th }}$ element in the specimen being analyzed is found from the relation

$$
\mathrm{C}_{i}=\mathbf{I}_{i}{ }^{*} / \mathbf{I}_{i}
$$

where $\mathrm{I}_{i}{ }^{*}$ and $\mathrm{I}_{i}$ are the intensities of the measured characteristic $\mathrm{x}-$ rays emerging from the specimen and the standard respectively. In the second approximation, where the differences of excitation and absorption of this radiation in the specimen and the standard are taken into account, an additional factor is introduced in eq. (1), which is called a correction to the results of the analysis.

Several empirical and semiempirical methods have been proposed to determine this correction (e.g., the review of Philibert (1965) [2]). The (semiempirical) nature of these methods of calculation is basically due to the complexity of the theoretical description of the distribution of electron scattering and deceleration in solids. Experiments indicate that the accuracy of currently used methods of calculation is not always satisfactory; therefore, further procedures are required.

The intensity of characteristic x-radiation emerging from the specimen is, in general, the sum of three terms corresponding to the excitation by electron collision (e), the fluorescence excited by characteristic radiation of other atoms (f), and by the bremsstrahlung emitted by the electrons (b), at frequencies above the absorption edge of the corresponding $\mathrm{q}_{i}$ level:

$$
\mathrm{I}_{i}^{*}=\mathrm{I}_{i e}{ }^{*}\left(1+\mathrm{I}_{i f}{ }^{*} / \mathrm{I}_{i e}{ }^{*}+\mathrm{I}_{i b} * / \mathrm{I}_{i e}{ }^{*}\right)
$$


When the fluorescent radiation excited by other elements is weak, the second term in (2) can be neglected (this term will be considered at the end of this paper); experiments also show that the third term in (2) is significant only for elements with high atomic number. In this work it is assumed that this term is negligibly small.

The expression for $\mathbf{I}_{i e}$ has the following form for massive specimens:

$$
I_{i e}=N_{o} N_{i} W_{i} \int_{0}^{\infty} \psi_{i e}(x) e^{-\mu_{i} x \operatorname{cosec} \theta} d x
$$

where $\mathrm{N}_{o}$ is the electron beam intensity impinging upon the sample, $N_{i}$ is the number of atoms of the $i^{\text {th }}$ element per unit volume of the specimen, $\mathrm{W}_{i}$ is the fluorescent yield in the $\mathrm{q}_{i}$ level, $\mu_{i}$ is the (linear) absorption coefficient of the respective radiation in the specimen, $\theta$ is the x-ray emergence angle, and $\psi_{i e}(\mathrm{x})$ is the distribution of the radiation excited by the electrons of the $\mathrm{q}_{i}$ level at the depth $\mathrm{x}$ of the specimen.

In this paper we describe a method for calculating $\psi_{i e}$ based upon the use of the law of attenuation of parallel monoenergetic electron beams in matter which was introduced in the publications of Makhov (1960) [3] and Dupouy and co-workers (1965) [4].

One can write $\psi_{i e}$ in the following general form:

$$
\psi_{i e}=\mathrm{S} \int_{\mathrm{E}_{i}}^{\mathrm{E}_{o}}\left(\mathrm{~F}_{i}(\mathrm{x}, \mathrm{E}, \phi) / \cos \phi\right) \sigma\left(\mathrm{E}, \mathrm{E}_{i}\right) \mathrm{dEd} \Omega
$$

in which $F_{i}(x, E, \psi)$ is the distribution of electrons according to energies $(E)$ and angle $(\phi)$ with respect to the initial direction at the depth $\mathrm{x}$ in the specimen; $\sigma\left(\mathrm{E}, \mathrm{E}_{i}\right)$ is the differential effective crosssection of excitation of the $\mathrm{q}_{i}$ level of characteristic radiation; $\cos \phi$ takes into account the effective path of electrons in the layer of material between the depths $\mathrm{x}$ and $\mathrm{x}+\mathrm{dx}$, which, due to scattering, is longer than the thickness of this layer; $E_{o}$ is the initial energy of the electrons, $E_{i}$ is the excitation energy of the $q_{i}$ level, and $s$ is a universal factor of proportionality.

The current theory of the scattering of electrons with energies of the order of $10^{3}-10^{4}$ electron volts does not allow the satisfactory calculation of the function $F_{i}(x, E, \phi)$ for all angles of scattering in the range $0 \leqslant \phi \leqslant \pi$, and for energies of the electrons such that $\mathrm{E}_{i} \leqslant \mathrm{E} \leqslant \mathrm{E}_{\theta}$. Therefore, in most publications (e.g. Castaing (1951) [1]) the following simplification is made:

$$
\left(\mathrm{F}_{i}(\mathrm{x}, \mathrm{E}, \phi) / \cos \phi\right) \rightarrow \mathrm{F}(\mathrm{E})
$$


That is, the angular and energy distributions of the electrons are neglected; the function $F(E)$ is considered to be universal and the difference in the conditions of electron deceleration in different elements is introduced as follows. One introduces (5) and (4) into (3) and disregards the absorption $\left(\mu_{i}-0\right)$; thus; one finds

$$
I_{i} \sim \int_{0}^{x_{i}} F \mathrm{dx}=\int_{\mathrm{E}_{o}}^{\mathrm{E}_{i}} \frac{\mathrm{F}(\mathrm{E}) \mathrm{dE}}{-(\mathrm{dE} / \mathrm{dx})_{i}}
$$

where $x_{i}$ is by definition the depth where $E\left(x_{i}\right)=E_{i}$. In this way, the differences which were pointed out above are transferred to the stopping power of the (target) material, $-(\mathrm{dE} / \mathrm{dx})_{i}$. Moreover, in this form the electrons which are backscattered do not appear in the calculation, and their contribution to $\psi_{i c}$ must be taken into account through an empirical factor $\lambda_{i}$ in (6) (Castaing (1960) [5], Poole and Thomas (1961) [6]). Philibert (1962) [7] attempted to empirically account for the backscattering of electrons and to obtáin a form of $\psi_{i e}$ closer to the experimental values (Castaing and Descamps (1955) [8]).

Both these methods have the disadvantage of neglecting the energy distribution of the electrons. In particular, the existence of this distribution leads to the fact that $\psi_{i e}\left(x_{>}>x_{i}\right) \neq 0$. In addition, the quantity $E$ in (6) is actually used in the sense of a mean energy of electrons; and while there is a theoretical expression (Bethe and coworkers (1930) [9]) for $\mathrm{dE} / \mathrm{dx}$, this, however, is valid only for the path length of electrons, so that $\mathrm{x}$ does not coincide with the thickness of the material along the original direction of the electron beam. Therefore, (6) is only a quite coarse approximation, even when the backscattered electrons are empirically taken into account. On the other hand, the method of Philibert ignores the fact that at the depth of (complete) diffusion, the angular distribution of electrons becomes isotropic, and therefore $\cos \phi$ in (4) cannot be considered to change continuously at all depths of $\mathrm{x}$, as Philibert assumes.

Rejecting all the assumptions which are not well founded, we introduce the following simplification. We divide the electron beam which passes through a section of the specimen into two parts: those moving in forward direction $\left(0 \leqslant \phi_{\eta} \leqslant \pi / 2\right)$ and those moving in backward direction $\left(\pi / 2 \leqslant \phi_{\delta} \leqslant \pi\right)$, each being characterized by its energy distribution and mean angular distribution:

$$
\frac{\mathrm{F}(\mathrm{x}, \mathrm{E}, \phi)}{\cos \phi} \rightarrow \frac{\mathrm{g}_{\eta}(\mathrm{x}, \mathrm{E})}{\overline{\cos \phi_{\eta}}(\mathrm{x})}+\frac{\mathrm{g}_{\delta}(\mathrm{x}, \mathrm{E})}{\overline{\cos \phi_{\delta}}(\mathrm{x})}
$$


where the indices $\eta$ and $\delta$ correspond to the forward and backward fluxes. In what follows, in the calculation of $\psi_{i e}$, we will use for $\cos \phi \eta(x)$ the expression obtained by Blanchard and Fano (1951) [10], and for the cross-section $\sigma\left(\mathrm{E}, \mathrm{E}_{i}\right)$ the expression proposed by Green and Cosslett (1961) [11]. Then, the calculation according to the scheme which we propose for the backscattering (mainly Rutherford scattering) shows that $\cos \phi \delta(x) \simeq$ const. $=1 / 2$.

Let us now turn to the determination of $g_{\eta}$ and $g_{\delta}$. To obtain $g_{\eta}$ we will use the law of electron attenuation of Makhov and Dupouy

$$
\eta(\mathrm{x})=\left(\mathrm{N}(\mathrm{x}) / \mathrm{N}_{o}\right)=\exp \left\{-(\mathrm{x} / \mathrm{X})^{p}\right\}
$$

where

$$
\mathrm{X}=\frac{a \mathrm{E}_{\mathrm{o}}^{\mathrm{n}}}{\rho}, \mathrm{n}=2.44\left(\frac{\mathrm{Z}}{\mathrm{A}}\right)^{1 / 2}, \mathrm{p}=\frac{1.51 \mathrm{n}}{\lg _{10} \mathrm{Z}}
$$

$\rho, \mathrm{A}$, and $\mathrm{Z}$ are the density, atomic weight, and atomic number of the material, and $\alpha=3.33 \times 10^{-6}$, when $\mathrm{E}_{o}$ is expressed in $\mathrm{kV}$, and $\mathrm{x}$ in $\mathrm{cm}$.

We note that the equation (8) can be obtained starting from the consideration that the linear attenuation coefficient of electrons in substances, $\mathrm{q}(\mathrm{x})$, is determined by the electron scattering processes which depend on the energy $\mathrm{E}(\mathrm{x})$. Writing the equation for the electron flux

$$
\mathrm{N}(\chi)=\mathrm{N}_{o}-\int_{0}^{\chi} \mathrm{N}(\chi) \mathrm{q}(\chi) \mathrm{d} \chi,
$$

where $\chi=\mathrm{x} / \mathrm{X}$, and assuming for the sake of simplicity that $\mathrm{q}(\chi)=$ $\mathrm{a} \chi^{b}$ where $\mathrm{a}$ and $\mathrm{b}$ are constants greater than zero, we find from (10) for the boundary condition $\mathrm{N}(0)=\mathrm{N}_{\mathrm{o}}$ :

$$
\eta(\chi)=\exp \left\{-\frac{\mathrm{a}}{\mathrm{b}-1} \chi^{b+1}\right\}
$$

whence assuming that $a=b+1=p$ we come to (8). The value of $p$ is thus larger than 1 for all elements, and this agrees with experiment (Dupouy et al. (1965) [4]).

To find $\mathrm{g}_{\eta}$, one must also know the law of electron deceleration.

Let us consider a group of electrons of energy $\mathrm{E}_{k}$, among the electrons which traverse the thickness $\mathrm{x}$ of the material. Let us call the maximum range of this group of electrons $x_{k} \geqslant x$. To determine the relation between $\mathrm{E}_{k}$ and $\mathrm{x}_{k}$. we will use a power law of deceleration of the form proposed by Dekker (1958) [12]:

$$
\mathrm{E}^{n}(\mathrm{x})=\mathrm{E}_{\mathrm{o}}{ }^{n}-\mathrm{cx}, \mathrm{c}=\mathrm{E}_{\mathrm{o}}{ }^{n / \mathrm{x}_{\max }}
$$


When this law is applied to the group of electrons with energy $\mathrm{E}_{k}$, we obtain

$$
\frac{\mathrm{E}_{k}{ }^{\mathrm{n}}}{\mathrm{E}_{\mathrm{o}}{ }^{\mathrm{n}}} \equiv \xi_{k}^{w}=1-\frac{\mathrm{x}}{\mathrm{x}_{k}}=1-\frac{X}{\chi_{k}} .
$$

Then, according to the definition of $\chi_{k}$.

$$
\eta\left(\chi_{k}\right)=\eta(\chi)-\int_{0}^{\xi_{k}} g_{\eta}(\chi, \xi) d \xi
$$

We find therefore that

$$
\mathrm{g} \eta=-\frac{\mathrm{d} \eta\left(\chi_{k}\right)}{\mathrm{d} \xi_{k}}=-\frac{\mathrm{d} \eta\left(\chi_{k}\right)}{\mathrm{d} \chi_{k}} \cdot \frac{\mathrm{d} \chi_{k}}{\mathrm{~d} \xi_{k}} .
$$

Using (8) and (12), and eliminating the index $\mathrm{k}$ due to its arbitrariness, for $\left(0 \leqslant \xi_{k} \leqslant 1\right)$, we find

$$
\begin{gathered}
g_{\eta}(\chi, \xi)=\operatorname{pn}_{\chi} \frac{\xi^{n-1}}{\left(1-\xi^{n}\right)^{p+1}} \exp \left\{-\left(\frac{\chi}{1-\xi^{n}}\right)^{p}\right\}, \\
\int_{0}^{1} g_{\eta}(\chi, \xi) \mathrm{d} \xi=\eta(\chi)
\end{gathered}
$$

To determine $\mathrm{g}_{\delta}$, let us consider the following calculations. A layer of material at depth from $\chi$ to $\chi+\mathrm{d} \chi$ attenuates the electron flux by $\mathrm{dN}(\chi)$. Calculations show that the probability of double backscattering at such depths, for $\mathrm{E} \sim 10^{3}-10^{4}$ is negligibly small, compared to the single backscattering in the layer between 0 and $\chi$. Because of this the backscattered fraction from the layer at depths $\chi, \chi+d \chi$ moving towards the surface of the specimen $(\chi=0)$ is mainly absorbed. Let $\mathrm{dN}_{\delta}(\chi)$ designate that fraction of the electrons which emerges through the surface of the specimen, and let a thickness $s \chi$ of the specimen $(s>1)$ be such that the attenuation of the beam $N(\chi)$ will there be the same (in relative units) as the attenuation of the (upward) beam of $d N(\chi)$. Then we may write

$$
\left(\mathrm{dN}_{\delta}(\chi) / \mathrm{dN}(\chi)\right)=(\mathrm{N}(\mathrm{s} \chi) / \mathrm{N}(\chi)) \text {. }
$$

Using for $N(\chi)$ the expression (8) and assuming for simplicity that $s$ is constant or that it is a slowly varying function of $\chi$, and also taking into account that the backscattering coefficient $\delta_{\infty}$ (for a thick 
specimen) tends to 0.5 with increasing $\mathrm{Z}(\mathrm{p} \rightarrow 1)$, we find that $\mathrm{s}=2$, and that

$$
\delta_{\infty}(\mathrm{Z})=0.5^{p(Z)}
$$

A comparison of $\delta_{\infty}(Z)$ from equation (17) with the results of the calculations of Everhart (1960) [13] and Archard (1960) [14], and with experimental determinations (Philibert $(1962,1964)$ [15]) is given in Figure 1. It is seen there that equation (17) gives a satisfactory agreement with the experiment for all values of $Z$.

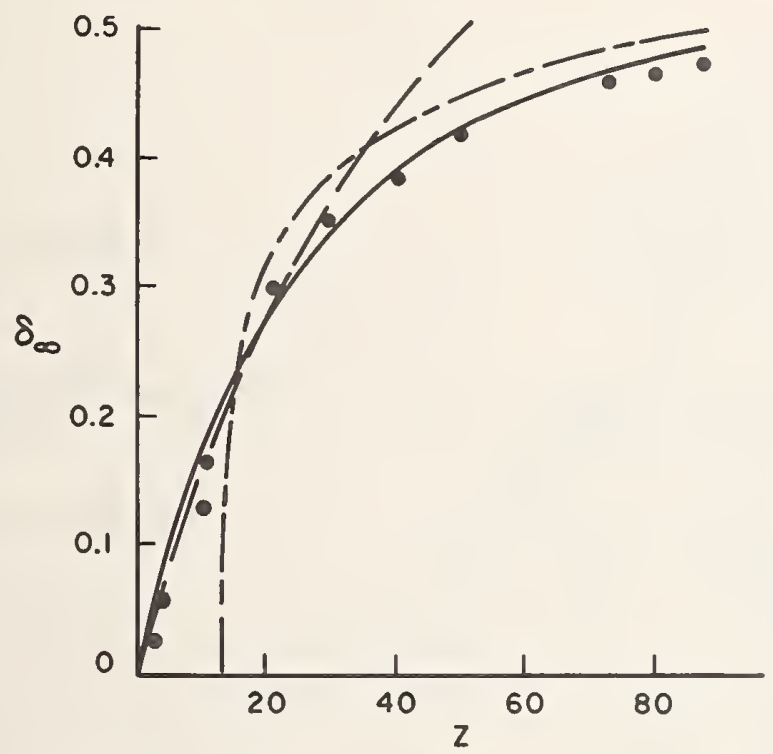

Figure 1. Dependence of the coefficient of backscattering of electrons, $\delta_{\infty}$, on the atomic number $\mathrm{z}$. : equation (17); -....-: Archard (1961) [14]; _ - -: Everhart (1960) [13].

Figure 2 shows the fraction of electrons $\Delta_{D}=1-\eta_{D}-\delta_{D}$, which is absorbed in the film of thickness $\mathrm{D}$, according to equations (8) and (16), and the corresponding experimental results obtained by Cosslett and Thomas (1964) [16]. These calculations allow us to conclude that the main contribution for the backscattering is due to the processes of elastic single scattering (Rutherford scattering) of electrons at angles $\phi>\pi / 2$. Hence, using the expression which was found with the aid of the equation (16) (when $s=2$ ) for the total 


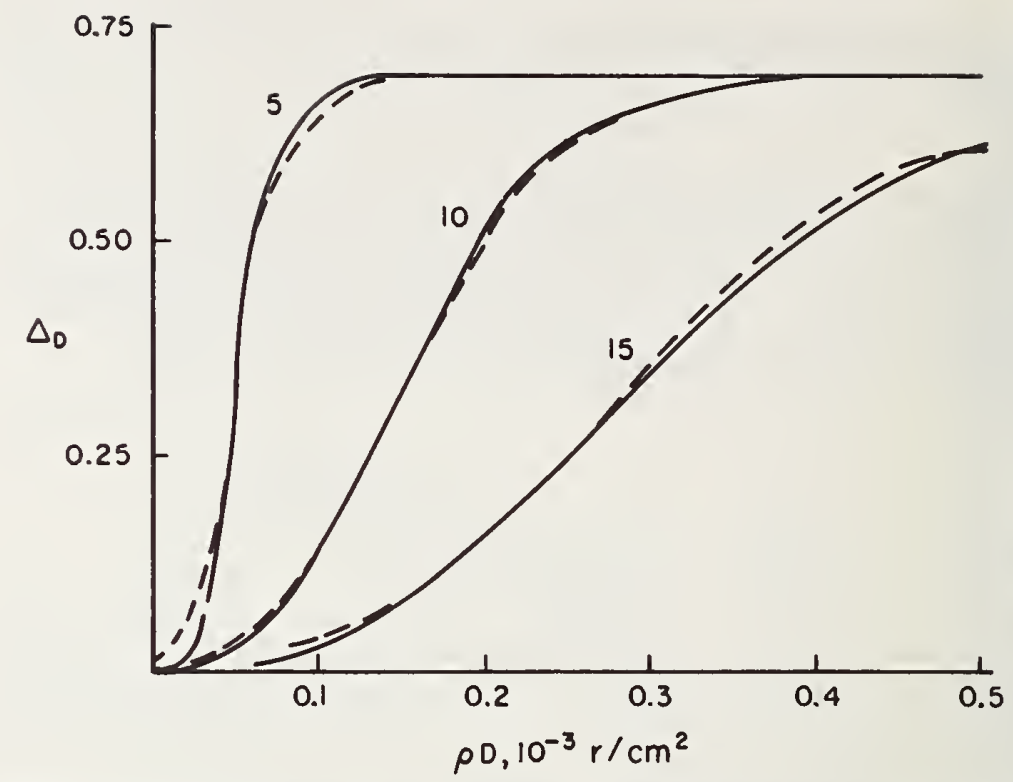

Figure 2. Fraction of electrons $\Delta_{l}=1-\eta_{D}-\delta_{D}$, which is absorbed in a copper film of thickness $D$, for $E_{o}=5,10,15 \mathrm{keV}$. (Thomas and Cosslett (1964) [16]).

number of backscattered electrons which pass through the section $\chi$ of the specimen, we write

$\delta(\chi)=\int_{\eta(\chi)}^{0} \frac{\eta(2 y-\chi)}{\eta(y)} d \eta(y)=p \int_{\chi}^{\infty} y^{p-1} \exp \left\{-(2 y-\chi)^{p}\right\} d y$

Using the energy distribution $g_{\eta}(15)$, we find the energy distribution of the backscattered electrons, $\mathrm{g}_{\delta}(\chi, \xi)$

$g \delta(\chi, \xi)=\int_{\eta(\chi)}^{0} \frac{d \delta(y)}{d \eta(y)} g(2 y-\chi, \xi) d \eta(y)=$

$=2 \mathrm{pn}^{2} \frac{\xi^{\mathrm{n}-1}}{\left(1-\xi^{\mathrm{n}}\right)^{\mathrm{p}+1}} \int_{\chi}^{\infty} \mathrm{y}^{\mathrm{p}-1}(2 y-\chi)^{\mathrm{p}} \exp \left\{-\left[1+\left(1-\xi^{\mathrm{n}}\right)^{\mathrm{p}}\right](2 y-\chi)^{\mathrm{p}}\right\} \mathrm{dy}$ 
In particular, for backscattered electrons which emerge from the surface of the specimen, we have

$\mathrm{g} \delta(\mathrm{O}, \xi)=\frac{\mathrm{pn}}{2^{\mathrm{p}-1}} \frac{\xi^{\mathrm{n}-1}\left(1-\xi^{\mathrm{n}}\right)^{\mathrm{p}-1}}{\left[1+\left(1-\xi^{\mathrm{n}}\right)^{\mathrm{p}}\right]^{2}}, \int_{0}^{1} \mathrm{~g}_{\delta}(\mathrm{O}, \xi) \mathrm{d} \xi=\delta_{\infty}$

A comparison of (20) with the experimental results for the determination of $\mathbf{g}_{\delta}$ (Brandt (1951) [17]) is given in Figure 3. We see that the agreement is quite satisfactory.

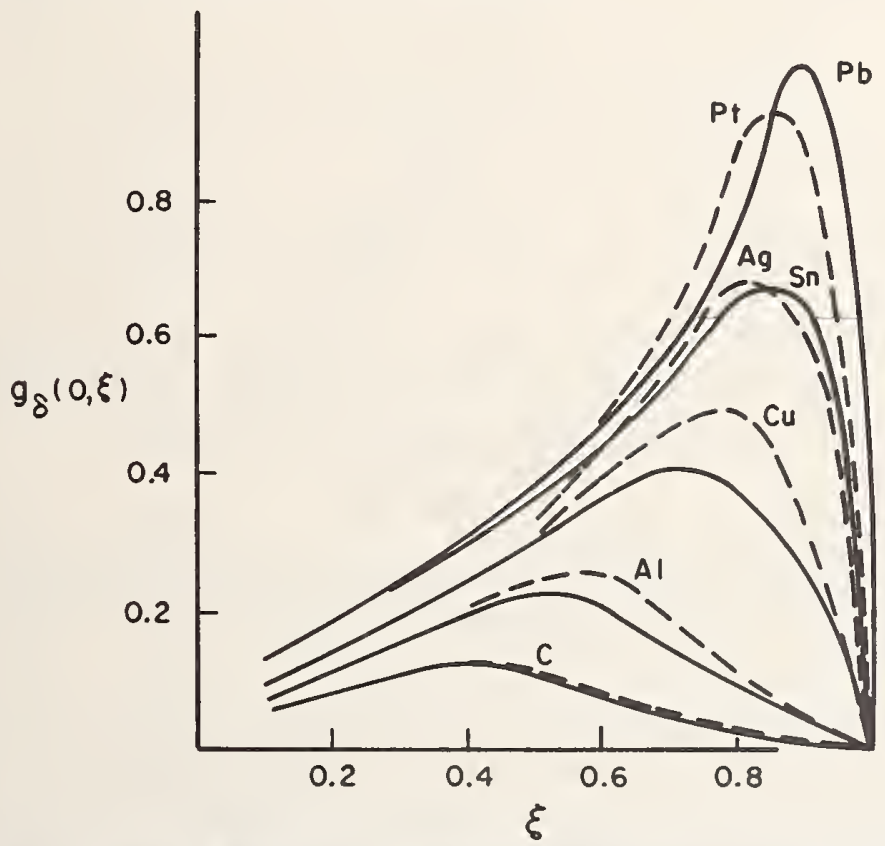

Figure 3. Energy distribution of backscattered electrons, $g(0, \xi)$ for various elements. : equation (20), -: experiment (Brand (1951) [17]).

We have calculated $g_{\eta}(15)$ and $g_{\delta}(19)$ for $p=2$ and inserted the results into (7) and then into (4). The choice $p=2$ is conditioned by the possibility of analytical calculation of the integral in (19) and also by the fact that this value of $p$ corresponds to $Z \approx 20$; in this region of $\mathrm{Z}$ we have experimental results available (Castaing and Descamps (1955) [8]) which allow us to carry out a comparison. This comparison is shown in Figure 4, where the upper curve (for $\mathrm{Cu}$ ) corresponds to $\mathrm{p}=1.7$, and the lower curve (for $\mathrm{Al}$ ) corresponds to 


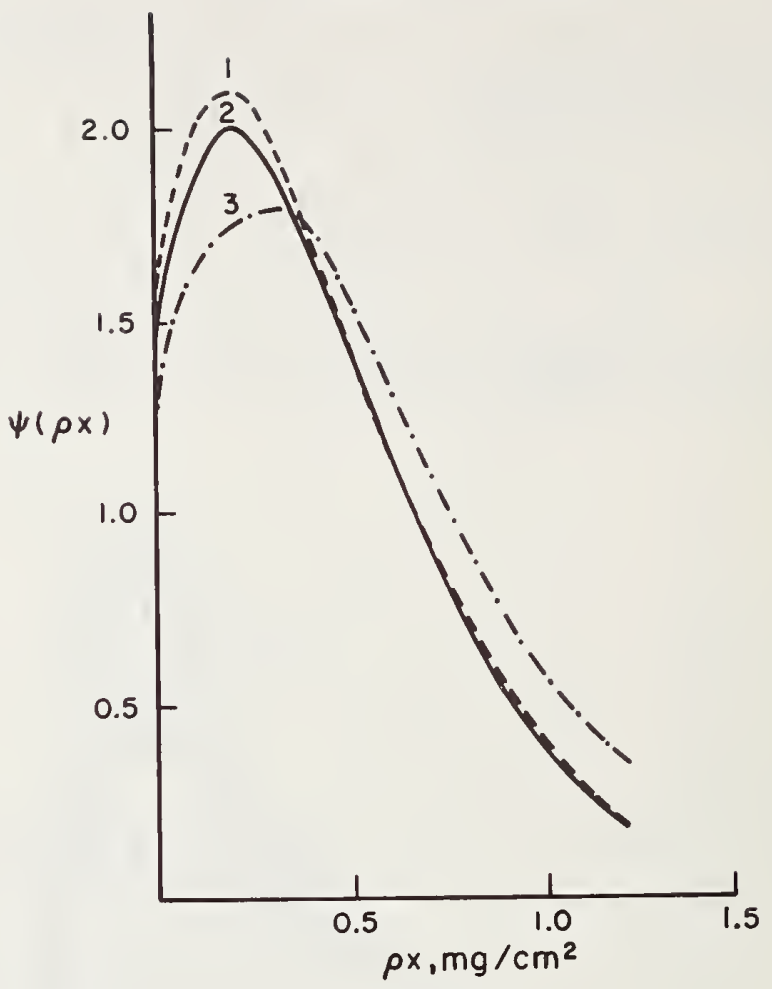

Figure 4. Distribution of the intensity of characteristic x-rays in a massive anode. 1,3: experiment (Castaing and Descamps (1955) [8]) for $\mathrm{Cu}$ and $\mathrm{Al} ; 2$ : theory, with $\mathrm{p}=2$.

$\mathrm{p}=2.3$. Figure 4 indicates a quite satisfactory agreement of the proposed calculation of $\psi_{i e}(\mathrm{x})$ with the experimental results.

However, for non-integer values of $p$ (equation 9) these calculations require the numerical integration of (4) and another numerical integration for the determination of $I_{i e}$ from (3). Therefore, there arises a need for an accurate and simple approximation to $\psi_{i e}$, which would allow us to carry out the calculation of $I_{i e}$ in an analytical form. The examination of experimental results for $\psi_{i e}$ allows us to conclude that their dependence on $\chi$ is quite similar over a wide range of $\mathrm{Z}$, and therefore of $p$, even including the case when $p$ tends to 1 . Moreover, $\psi_{i e}$ appears only under the integral sign; and this permits us to introduce a simple function which compensates for the hump of $\psi_{i e}$ 
at small values of $\chi$ and for the rapid $\left(\sim \exp \left(-\chi^{p}\right)\right)$ decrease of $\psi_{i e}$ at large values of $\chi$.

Starting from these considerations, we will choose a tentative approximation for $\psi_{i e}$ in the following form:

$$
\psi_{i e} \sim \psi_{i e}(0) \exp \left\{-\frac{x}{1-\xi_{i}^{n}}\right\}
$$

where $\xi_{i}=\mathrm{E}_{i} / \mathrm{E}_{o}$ and $\chi$ and $\mathrm{n}$ are calculated from (8) and (9). The definition of $\psi_{i e}(0)$ according to (4), (7), (15), and (20) yields the following:

$$
\begin{aligned}
& \psi_{\text {ie }}(0)=\frac{\ln \beta}{\beta}+2 \int_{1 / \beta}^{1} \mathrm{~g}_{\delta}(0, \xi) \sigma\left(\xi, \xi_{i}\right) \mathrm{d} \xi \approx \\
& \approx \frac{\ln \beta}{\beta}\left\{1+2 \delta_{\infty}\left(1-\beta^{-n)^{p}}\right\}\right.
\end{aligned}
$$

where $\beta=1 / \xi_{i}$. A comparison of the results of calculating $\psi_{i e}(0)$ according to (22) with the experiment is given in Table I.

\section{TABLE 1}

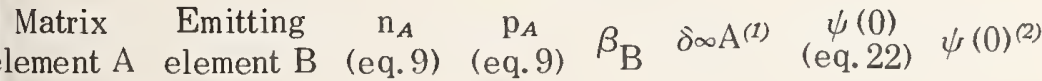

$\begin{array}{llllllll}\mathrm{Au} & \mathrm{Bi} & 1.55 & 1.22 & 2.18 & 0.44 & 1.57 & 1.61 \\ \mathrm{Cu} & \mathrm{Zn} & 1.65 & 1.70 & 3.01 & .30 & 1.46 & 1.475 \\ \mathrm{Al} & \mathrm{Cu} & 1.70 & 2.30 & 3.21 & .135 & 1.19 & 1.16\end{array}$

Philibert $(1962,1964)[15]$.

${ }^{2}$ Castaing and Descamps (1955) [8].

Substituting (21) and (22) into (3), we can obtain the dependence of the intensity of the characteristic radiation of a pure element $\mathbf{I}_{i e}$ on the overvoltage $\beta=\mathrm{E}_{o} / \mathrm{E}_{i}$, the (mass) absorption coefficient $\tau_{i}=$ $\mu_{i} / \rho_{i}$, and the emergence angle of this radiation with respect to the specimen surface:

$$
\mathrm{I}(\beta, \tau, \theta) \sim\left[1+2 \delta_{\infty}\left(1-\beta^{-\mathrm{n}) \mathrm{p}}\right] \frac{\beta^{\mathrm{n}}-1}{1+\mathrm{Q}\left(\beta^{\mathrm{n}}-1\right)} \cdot \frac{\ln \beta}{\beta} .\right.
$$


In this equation, $\mathrm{Q}=\alpha \mathrm{E}_{i}{ }^{n} \tau_{i} \operatorname{cosec} \theta$. When $\beta \gg 1$, the relation (23) is reduced to the following form:

$$
\mathrm{I}(\beta) \sim\left(\ln \beta \cdot \beta^{n-1}\right) /\left(1+\mathrm{Q} \beta^{n}\right)
$$

and it is characterized by a maximum at $\beta_{m}$, which is determined by the equation

$$
\ln B_{-}=\left(1-\frac{\mathrm{n}}{1+\mathrm{Q} \beta_{m}^{\mathrm{n}}}\right)^{-1}>0
$$

The dependence of $\mathbf{I}(\tau, \theta)$ for $\beta=$ const. has the following form:

$$
\mathrm{I}(\tau, \theta) \sim \frac{1}{1+\mathrm{Q}\left(\beta^{\mathrm{n}}-1\right)}
$$

We checked the expressions (24) and (25) over a wide range of $\beta$ and $\mathrm{Z}$. The comparison of (24) with the experiment (Campbell (1963) [18]) for $\mathrm{Be}, \mathrm{B}$, and $\mathrm{C}$ (in these cases $\mathrm{p}>3$ ) is shown in Figure 5 , and the comparison of (25) with the determination of $\mathrm{I}(\tau, \theta)$

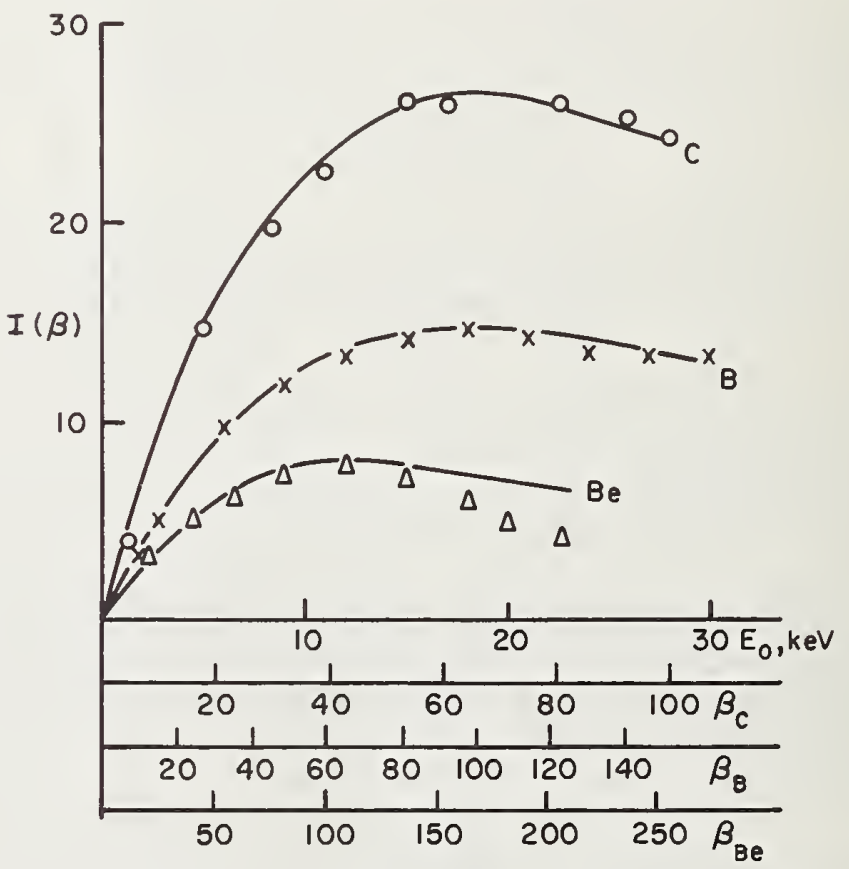

Figure 5. Dependence of $I(\beta)$ for $K$ radiation of light elements. Solid curves represent equation (24); points represent the experiment of Campbell (1963) [18]. 
for aluminum, for different values of $\beta$ (Green (1964) [19]) in Figure 6. In all these cases a quite satisfactory agreement of theory and experiment is observed.

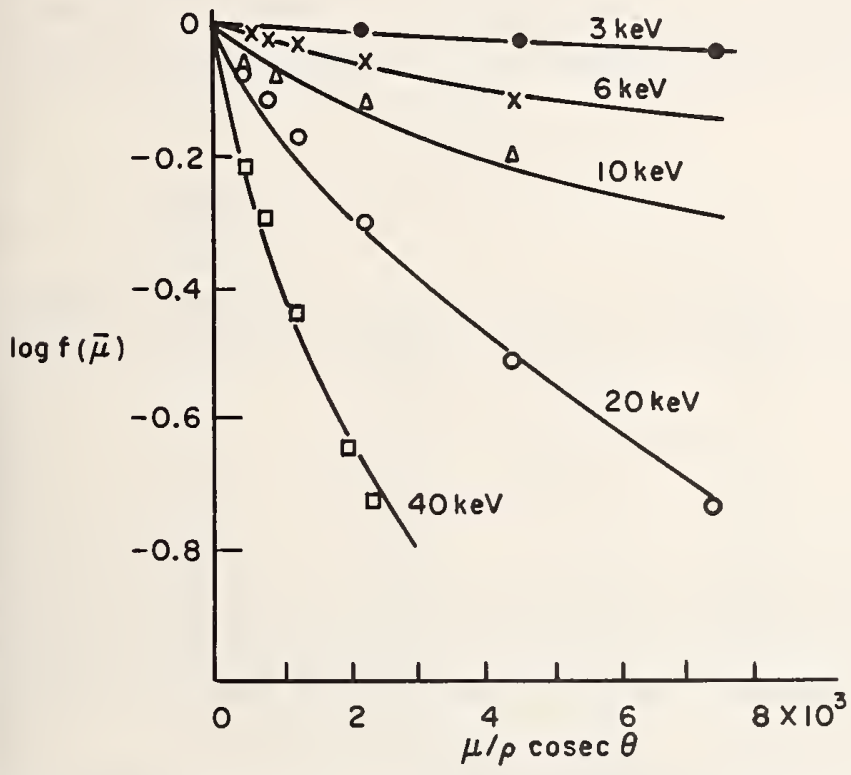

rigure 6. The dependence of $\mathrm{I}(\mu, \theta)$ for $\mathrm{Al} \mathrm{K} \alpha$ radiation, for various values of $\mathrm{E}_{o}$ : equation (25), points represent the experiment (Green (1963) [19]).

Equation (23) can be useu to introduce corrections which take into account the atomic number effect and the absorption (of $\mathrm{x}$-rays) in the calculation of the results of microprobe analyses using pure elements as external standards. In this case, we have

$$
\mathrm{k}_{i}=\mathrm{I}_{i}{ }^{*} / \mathrm{I}_{i}=\mathrm{c}_{i} \mathrm{R}_{i}^{(1)} \mathrm{R}_{i}^{(2)},
$$

where

$$
\begin{aligned}
& \mathrm{R}_{i}^{(1)}=\frac{1+2 \delta_{\infty}^{*}\left(1-\beta^{-\mathrm{n}}\right)}{1+2 \delta_{\infty}\left(1-\beta^{-\mathrm{n}}\right)}, \\
& \mathrm{R}_{i}^{(2)}=\frac{1+\alpha \mathrm{E}_{o}^{\mathrm{n}} \tau_{i i}\left(1-\beta^{-\mathrm{n}}\right) \operatorname{cosec} \theta}{1+\alpha \mathrm{E}_{o}^{\mathrm{n}} \tau_{i}^{*}\left(1-\beta^{-\mathrm{n}}\right) \operatorname{cosec} \theta}
\end{aligned}
$$

where $\delta_{\infty}^{*}=\sum_{i} \mathrm{c}_{i} \delta_{\infty_{i}}$ (Castaing (1960) [5]), $\tau_{i}^{*}=\sum_{j} \mathrm{c}_{j} \tau_{i j}$, 
$\tau_{i j}$ being the absorption coefficient for absorption of $\mathrm{q}_{\mathrm{i}}$-radiation of element $\mathrm{i}$ by element $\mathrm{j}$. In accordance with the approximation (21) we chose for $n$ a value equal to 1.62 (Dupouy et al. (1965) [4]).

For elements of relatively close atomic ntmber $\mathrm{Z}$, the factor $\mathrm{R}_{i}{ }^{(1)}$ $=1$, within limits of experimental error. Thus, (in absence of fluorescent excitation)(26) reduces to a linear system of equations:

$$
\mathrm{k}_{i}=\frac{\mathrm{c}_{i}\left(1+\mathrm{q}_{i} \tau_{i j}\right)}{\sum_{j} \mathrm{G}_{j}\left(1+\mathrm{q}_{j} \tau_{i j}\right)}
$$

in which $\mathrm{q}_{i}=\alpha\left(\mathrm{E}_{o}{ }^{n}-\mathrm{E}_{i}{ }^{n}\right) \operatorname{cosec} \theta$. This system can be solved without difficulties. In particular, for binary systems of elements, we have

$$
\mathrm{C}_{i}=\frac{\mathrm{k}_{i}\left(1+\mathrm{q}_{i} \tau_{i j}\right)}{\mathrm{q}_{i}\left[\mathrm{k}_{i} \tau_{i j}+\left(1-\mathrm{k}_{i}\right) \tau_{i j}\right)}
$$

We shall give here the results of comparing equation (26) with experiment, including limiting cases in which either $R_{i}^{(1)}$ or $R_{i}^{(2)}$ are, for all practical purposes, equal to one. A comparison of the data given by Poole and Thomas (1961) [6], and Ziebold and Ogilvie (1963) [22] with our own results is given in Table 2.

A comparison of results of the determination of $\mathrm{C}_{\mathrm{r} e}$ in the system $\mathrm{Fe}-\mathrm{Cr}$, according to equation (28), with the data of Colby and Conley (1965) [20] is shown in Figure 7, where we also present the results of the calculation according to Duncumb and Shields (1965) [2I], and to Ziebold and Ogilvie (1963) [22]. It is seen that the precision of equation (28) is compatible to that of the method of Duncumb and Shields when $\mathrm{C}_{\mathrm{Fe}}$ is small, and somewhat better when $\mathrm{C}_{\mathrm{Fe}}$ is large. The fact that these relations show the same type of divergence from the experimental values when different assumptions are being used indicates that there is probably some systematic error in the measurements of $\mathrm{k}_{r^{\prime} e}$ by Colby and Conley.

In the case of many-element specimens in which there is considerable (fluorescent) excitation of the radiation of the level $\mathrm{q}$, by the characteristic radiation of other elements with $\nu_{j}>\mathrm{E}_{i} / \mathrm{h}$, the second term in (2) must be taken into account. We use an expression obtained by Castaing (1951) [1] which is the most accurate of all those proposed (see Duncumb and Shields (1963) [23]); but we will slightly change several terms. Instead of the energy factor $\mathrm{E}_{i} / \mathrm{E}_{j}$ we shall use the excitation cross-section in the form proposed. 
by Green and Cosslett (1961) [11]; thus we obtain

$$
\frac{\mathrm{E}_{i}}{\mathrm{E}_{j}} \cdot \frac{\ln \left(\mathrm{E}_{o} / \mathrm{E}_{j}\right)}{\ln \left(\mathrm{E}_{o} / \mathrm{E}_{j}\right)}
$$

TABLE 2

\begin{tabular}{|c|c|c|c|c|c|}
\hline System & $\begin{array}{l}\text { Element } \\
\text { being } \\
\text { analyzed }\end{array}$ & $\mathrm{C}_{\text {chem. }}$. & $\mathrm{k}$ & $\begin{array}{c}\mathrm{C} \\
\text { (eq. 26) }\end{array}$ & $\Delta \mathrm{C} / \mathrm{C} \%$ \\
\hline $\mathrm{Nb} / \mathrm{Be}$ & $\mathrm{Nb}$ & 0.462 & 0.454 & 0.460 & -0.4 \\
\hline riO & $\mathrm{Ti}$ & .750 & .722 & .745 & -.7 \\
\hline $\mathrm{Au} / \mathrm{Mn}$ & $\mathrm{Au}$ & .765 & .683 & .735 & -4.0 \\
\hline $\mathrm{UO}_{2}$ & $\mathrm{U}$ & .881 & .837 & .895 & +1.6 \\
\hline $\mathrm{ZrSi}_{2}$ & $\mathrm{Zr}$ & .620 & .544 & .578 & -6.8 \\
\hline $\mathrm{Lu} / \mathrm{Au}$ & $\mathrm{Au}$ & .238 & .174 & .235 & -1.2 \\
\hline $\mathrm{Cu} / \mathrm{Al}$ & $\mathrm{Cu}$ & .536 & .496 & .539 & +.6 \\
\hline $\mathrm{Zr} / \mathrm{O}$ & $\mathrm{Zr}$ & .945 & .920 & .935 & -1.1 \\
\hline $4 u / G a$ & $\mathrm{Au}$ & .739 & .718 & .725 & -1.9 \\
\hline $\mathrm{Fe}_{2} \mathrm{O}_{3}$ & $\mathrm{Fe}$ & .700 & .653 & .704 & -.6 \\
\hline \multirow[t]{4}{*}{$\mathrm{Ni} / \mathrm{Pt}$} & $\mathrm{Ni}$ & .551 & .560 & .555 & +.73 \\
\hline & & .297 & .305 & .296 & -.34 \\
\hline & & .164 & .166 & .162 & -1.22 \\
\hline & & .065 & .067 & .066 & +1.5 \\
\hline \multirow[t]{4}{*}{$\mathrm{Au} / \mathrm{Cu}$} & $\mathrm{Au}$ & .206 & .152 & .198 & -3.88 \\
\hline & & .402 & .324 & .403 & +.25 \\
\hline & & .601 & .505 & .602 & +.16 \\
\hline & & .799 & .730 & .799 & 0 \\
\hline \multirow[t]{7}{*}{$\Gamma \mathrm{a} / \mathrm{Ru}$} & $\mathrm{Ta}$ & .165 & .160 & .175 & +6.1 \\
\hline & & .309 & .289 & .311 & +.65 \\
\hline & & .434 & .414 & .439 & +1.14 \\
\hline & & .544 & .532 & .558 & +2.57 \\
\hline & & .642 & .635 & .662 & +3.12 \\
\hline & & .807 & .800 & .812 & +.62 \\
\hline & & .941 & .960 & .963 & +2.34 \\
\hline
\end{tabular}

f This, in accordance with the experiment, slightly decreases the f correction for fluorescence. The improvement obtained by inserting $\sigma^{1}=\left[\alpha\left(\mathbf{E}_{0}{ }^{n}-\mathbf{E}_{j}{ }^{n}\right)\right]^{-1}$ instead of the constant of Lenard $(\sigma)$ in the log term $\mathrm{v}=\sigma / \rho \tau_{j}^{*}$, which follows from the law of attenuation in lequation (8), is less important. Finally, the possible difference between the $q_{j}$ level of the spectral series which causes the 

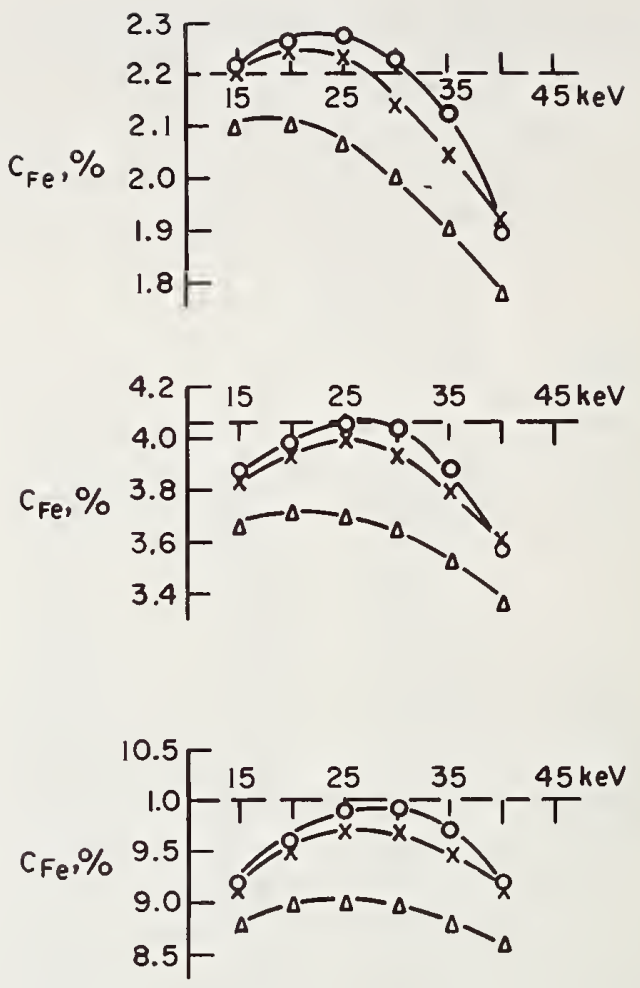

Figure 7. Concentration $\mathrm{C}_{\mathrm{Fe}}$ in the $\mathrm{Fe}-\mathrm{Cr}$ system as calculated from the values of $\mathrm{k}_{\text {re }}$ measured by Colby and Conley (1965) [20]. o: equation (26), $\mathrm{x}$ : Duncumb and Shields (1965) [21], $\Delta$ : Ziebold and Ogilvie (1963) [22].

excitation and the $q_{i}$ level which is excited can be taken into account following the procedure of Reed (1965) [24]. Figure 8 shows the improvement of the correction for fluorescence in $\mathrm{C}_{{ }^{\prime} r}$ in the system $\mathrm{Fe}-\mathrm{Cr}$ which is obtained when these modifications are introduced, using the data of Colby and Conley (1965) [20] of $\mathrm{k}_{\mathrm{cr}}$. For comparison, we also give there the results of calculations by the unmodified equation of Castaing and by the equation of Ziebold and Ogilvie (1963) [22] and Wittry (1961) [25].

In general, the equations (2) constitute a substantially non-linear system which can be solved only by successive approximations. Due to the large volume, such calculations can be carried out only on an electronic computer. 

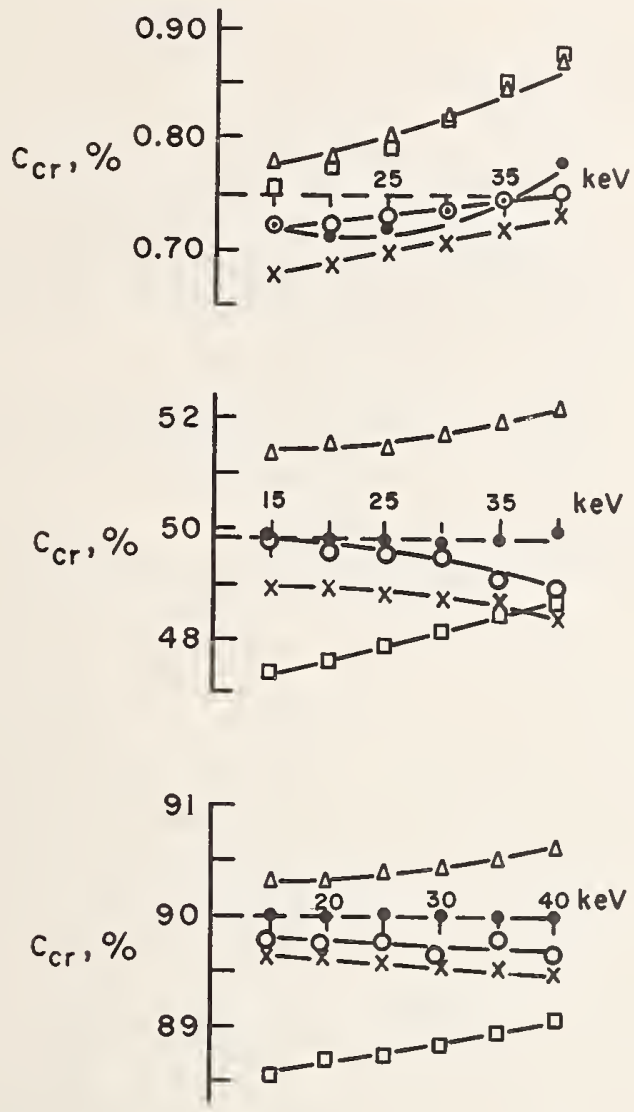

Figure 8. Concentrations $\mathrm{C}_{(r}$ in the system $\mathrm{Fe}-\mathrm{Cr}$, calculated from the values of $\mathrm{k}_{(r}$ measured by Colby and Conley (1965) [20]. o: theory, $\bullet$ : Castaing (1951) [1]. with improvement according to (29), x: Castaing (1951) [1], $\Delta$ : Ziebold and Ogilvie (1963) [22], 口: Wittry (1961) [25].

\section{REFERENCES}

1] Castaing, R., Thesis, Paris (1951).

2] Philibert, J., Publ. IRSID, Ser. B, No. 51 (1965).

3] Makhov, A., Fiz. Tverd. Tela 2,2161 (1960).

4] Dupouy, G., et al., Compt. Rend. 260,6055 (1965).

5] Castaing, R., Adv. Electron. Phys. 13, 317 (1960).

6] Poole, D., Thomas, A., Rep. AERE R-3815 (1961).

7] Philibert, J., X-ray Optics and X-ray Microanalysis p. 361 (1963).

8] Castaing, R., Descamps, J., J. Phys. Rad. 16, 304 (1955).

9] Bethe, H., Ann. der Physik 5, 325 (1930). 
[10] Blanchard, C., Fano, U., Phys. Rev. 82, 767 (1951).

[11] Green, M., Cosslett, V., Proc. Phys. Soc. 78, 1206 (1961).

[12] Dekker, A., Solid State Physics 6, 251 (1958).

[13] Everhart, T., J. Appl. Phys. 31, 1483 (1960).

[14] Archard, G., J. of Appl. Phys. 32, 1505 (1961).

[15] Philibert, J., Weinryb, E., C. R. 255, 1757 (1962), C. R. 258, 4535 (1964).

[16] Cosslett, V., Thomas, R., Brit. J. Appl. Phys. 15, 883 (1964).

[17] Brand, A., Proc. 12th Ann. Conf. Phys. Electronics, p. 74 (1952).

[18] Campbell, A., Proc. Roy. Soc. A274, 319 (1963).

[19] Green, M., Proc. Phys. Soc. 83, 435 (1964).

[20] Colby, J., Conley, D., NLCO Rep. No. 960 (1965).

[21] Duncumb, P., Shields, P., Tube Invest. Res. Lab. Rep. No. 181 (1965).

[22] Ziebold, T., Ogilvie, R., Anal. Chem. 35, 621 (1963).

[23] Duncumb, P., Shields, P., X-ray Optics and X-ray Microanalysis, New Yorl Acad. Press, p. 329 (1963).

[24] Reed, S., Brit. J. Appl. Phys. 16,913 (1965).

[25] Wittry, D., J. Appl. Phys. 29, 1543 (1958). 


\section{ON THE STRUCTURE OF FORMULAS FOR QUANTITATIVE ANALYSIS}

\section{JOHN CRISS}

Naval Research Laboratory, Washington, D.C.

\section{Abstract}

Two mathematically equivalent forms for expressing total haracteristic $\mathrm{x}$-ray emission are discussed. It is shown that the ommon practice of representing the various physical effects as a equence of correction factors has led to approximations and ssociated errors. The less common formulation, which expresses tal x-ray emission as a sum of several contributions, would be qually convenient for computation, if the approximations were not hade. A new formula is proposed, to express fluorescence by haracteristic lines in terms of $\mathrm{f}(\chi)$. As an example, Philibert's $\mathrm{f}(\chi)$ vas used to compare this formula with the mathematical form btained by Castaing and others. Using the same values for uorescence yield, absorption coefficients, and all other undamental parameters, the two expressions can differ by more han $6 \%$ in their predictions of the fluorescence contribution.

\section{Introduction}

This discussion is essentially an essay, in that it represents a ersonal point of view and makes no attempt at completeness. The rst half includes an interpretation of the basic problem of uantitative analysis and some general comments on approaches to he problem of developing convenient formulas. The second half ompares an equation written in terms of correction factors with one hat expresses total $\mathrm{x}$-ray intensity as a sum of primary and econdary contributions. A new formula is proposed, to express luorescence by characteristic lines in terms of $\mathrm{f}(\chi)$. This formulation voids the previous necessity of approximating $\phi(\rho z)$ and provides convenient means of improving the fluorescence correction as etter expressions for $\mathrm{f}(\chi)$ become available. 


\section{Discussion}

\section{A. PROBLEM}

The fundamental premise of quantitative electron probe analysi is that, given a set of operating conditions and a measure characteristic $x$-ray emission spectrum, there can be only on composition for the specimen.

The basic problem is to discover this functional relationship, an to express it in a form that permits calculation of specime composition. Ideally, one would have an explicit equation -

Mass concentration of a particular element $=$ some function of: relevant properties of all elements in specimen, measured characteristic $x$-ray intensities, properties of the apparatus, operating conditions, and other environmental factors.

Failing the discovery of such an explicit expression, one would hav to use an implicit formulation, from which specimen compositio might be determined by a number of techniques.

The problem involves, first of all, identifying those features tha affect the experimental results, and that also vary within the scop of the desired formulation. The second part of the problem involve expressing our understanding of the effects of all relevant variables.

The essential requirement is to express the effects of changes $i$ various parameters, and not necessarily to express all the physic interactions through which these effects arise. In other words, theris nothing wrong with an empirical method that work Unfortunately, the empirical methods suggested to date generall require the analysis of several standards and usually are of limite applicability.

\section{B. APPROACHES}

It is the failure of empirical approaches, together with our desi to understand and formulate the physics involved, that requires us express the effects of different parameters in terms of o knowledge of more fundamental physical processes. A "bru force" approach that, in principle, would solve the problem wou be a complete Monte Carlo treatment. A large number of electrol would be followed through every interaction until they either hi escaped the specimen or had lost sufficient energy to be no longer interest. All the consequences of these interactions wou themselves be described, and the final effects of interest would 1 
alculated. The disadvantages of such a treatment include the normous number of calculations needed to insure statistically good esuits and, more seriously, the huge quantity of fundamental nformation required to describe all the individual interactions. ufficiently accurate data simply do not exist.

It is true that if we really understood all the fundamental physical nteractions quantitatively, then we could calculate composition rom relative $x$-ray intensity. However, it does not follow that to chieve any specified finite accuracy we need to understand all the hysical interactions. Such complete understanding is an ultimate oal, but our present analysis techniques must be based on whatever formation is available. The degree to which we analyze physical rocesses, and the kinds of interactions on which our calculations re based, should depend upon whether the ultimate interactions are ny better understood than their grosser effects, and whether the ombined effects of these ultimate interactions really can be xpressed and calculated.

When suitable simplifying assumptions are made, then Monte farlo calculations become much less formidable and numerical blutions of a transport equation become feasible. In addition, fewer hput data are required for such treatments. The accuracy of the esults depends on the accuracy of the input data, the aptness of the athematical model, and the precision of the computational chnique.

The results of interest should be checked with experiment. If the greement is not satisfactory, then the mathematical model might be rodified and more accurate input data might be sought. If the esults of our most elaborate calculations, using the best input data vailable, still do not match our observations within the xperimental error, then we probably will try to adjust our alculations in a manner that would insure agreement. Thus we ould make our method partly empirical.

If we are interested primarily in the physics of $x$-ray production, en an empirical adjustment would be unappealing; we would refer to let experiment determine fundamental parameters (such as arious cross-sections), rather than affect our results more directly.

However, if our main concern is to calculate the composition of ectron probe specimens, then we would welcome any method, to hatever extent empirical, that would be widely applicable and that ould give good answers. Of course, we also want calculations that re simple. 


\section{DEVELOPMENT OF FORMULAS}

One very great advantage of the fundamental approaches is that they can lead to convenient formulas. Suppose a method of calculation, perhaps very complicated, has been tested experimentally for several widely different situations. This method could be used to generate values of compositions and corresponding $x$-ray intensities for a greater variety of situations than experiment alone could feasibly provide. Such information could be used to check the results of more approximate treatments. It might alsc happen that simpler theoretical approaches could provide : mathematical frame into which could be substituted parameters calculated solely to make the simpler formula agree with results of the more elaborate calculations.

As suggested earlier, the real test of a formula is whether the results of direct interest in quantitative analysis agree witl experience. To check less essential implications of a method migh be too severe a test. An example is provided by Philibert's

$$
f(\chi)=\frac{1+h}{\left(1+\chi^{/ \sigma}\right)\left[1+h\left(1+\chi^{/ \sigma}\right)\right]}
$$

This representation corresponds to an electron excitation functio of the form

$$
\phi(\rho z)=(\text { const. }) \times\left\{\exp [-\sigma \rho \mathrm{z}]-\exp \left[-\frac{1+\mathrm{h}}{\mathrm{h}} \quad \sigma \rho \mathrm{z}\right]\right\},
$$

which may be verified by calculating

$$
F(\chi)=\int_{0}^{\infty} \phi(\rho z) \exp [-\chi \rho z] d \rho z
$$

and

$$
f(\chi)=F(\chi) / F(0)
$$

An interesting feature is that

$$
\phi(0)=0
$$

regardless of the value of the constant. Such obvious disagreeme with measurements of $\phi(\rho z)$ (Fig. 1) should not in itself be criticism of the formula for $f(\chi)$. Philibert's equation (especially wi the $\sigma$ as modified by Duncumb and Shields, and others) has enjoys wide applicability and reasonable accuracy. 


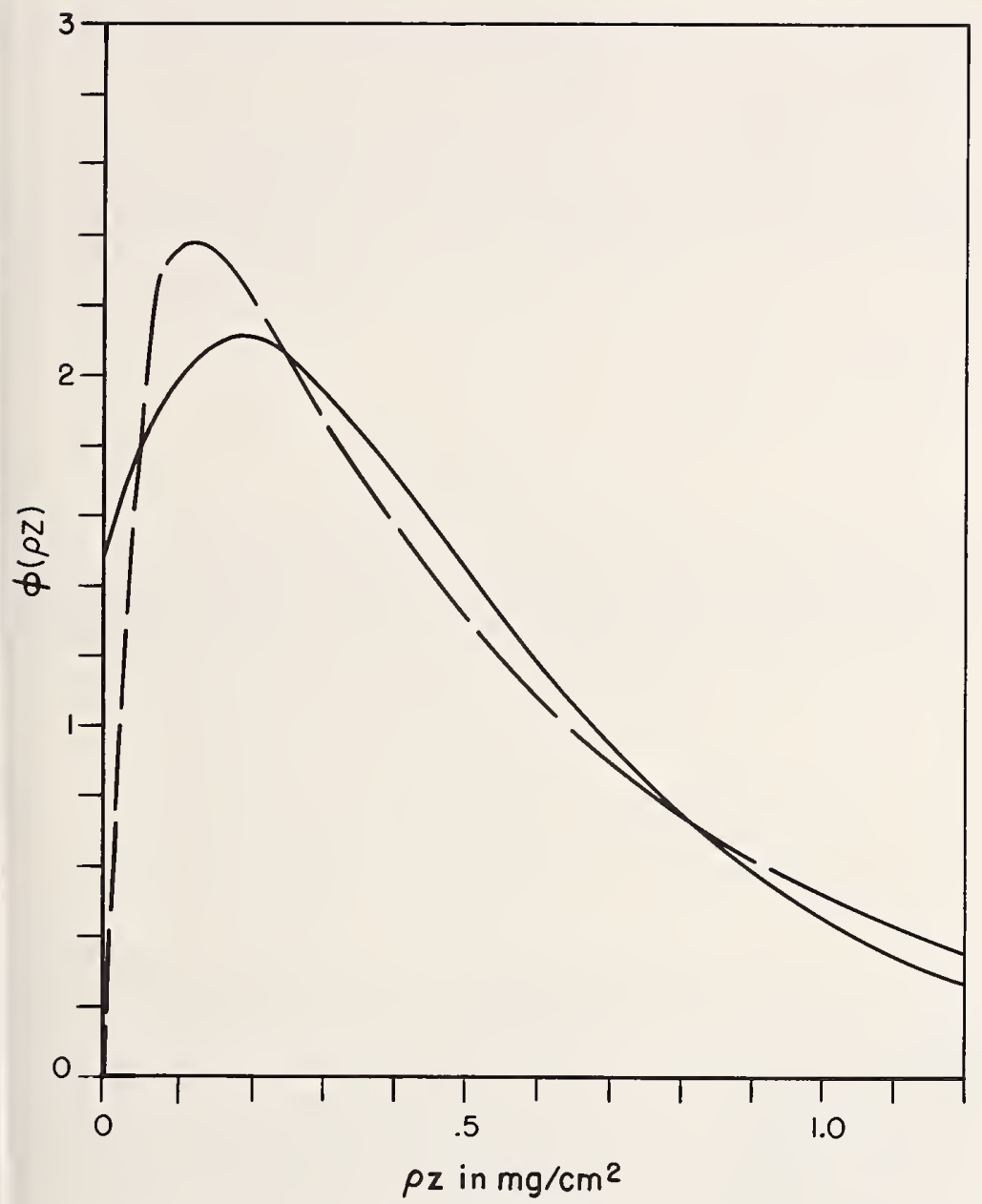

Figure 1. Comparison of two $\phi(\rho \mathrm{z})$ curves for copper at $29 \mathrm{keV}$. The solid line is the measurement by Castaing and Descamps. The dashed line corresponds to Philibert's $f(\chi)$ with the Duncumb-Shields $\sigma$. The curves are normalized to give equal values for $F(0)$.

\section{STRUCTURE OF FORMULAS}

Whether one used elaborate calculations or a partially empirical technique, there would seem to be an advantage in organizing the available information in a way that treated individually those contributions that could be found separately. 
It has been customary to consider separately the following three sources of characteristic radiation:

(1) ionization by the incident electrons,

(2) ionization by other characteristic radiation, and

(3) ionization by the Bremsstrahlung.

If these components are labeled $I_{1}, I_{2}$, and $I_{3}$, then the total intensity is

$$
\mathrm{I}=\mathrm{I}_{1}+\mathrm{I}_{2}+\mathrm{I}_{3}
$$

and relative intensity is

$$
\frac{\mathrm{I}}{\mathrm{I}^{*}}=\frac{\mathrm{I}_{1}+\mathrm{I}_{2}+\mathrm{I}_{3}}{\mathrm{I}_{1}^{*}+\mathrm{I}_{2}^{*}+\mathrm{I}_{3}^{*}}
$$

where the asterisk refers to the comparison standard.

In spite of the simplicity of this last equation, the relation often is expressed in terms of factors:

$$
\frac{\mathrm{I}}{\mathrm{I}^{*}}=\left(\frac{\mathrm{I}_{1}}{\mathrm{I}_{1}^{*}}\right) \frac{\left(1+\frac{\mathrm{I}_{2}}{\mathrm{I}_{1}}\right)\left(1+\frac{\mathrm{I}_{3}}{\mathrm{I}_{1}+\mathrm{I}_{2}}\right)}{\left(1+\frac{\mathrm{I}_{2}^{*}}{\mathrm{I}_{1}^{*}}\right)\left(1+\frac{\mathrm{I}_{3}^{*}}{\mathrm{I}_{1}^{*}+\mathrm{I}_{2}^{*}}\right)}
$$

These two forms are mathematically equivalent, and the choice between them could be a matter of personal preference. The firs factor in equation (8) usually has been written as the product of ar "atomic number factor" and an "absorption correction factor". The remaining factors are "fluorescence correction factors" fo" excitation by other characteristic lines and by the continuum.

Each correction factor supposedly accounts for the effect of different type of physical interaction. The atomic number factor is fashioned so that it would equal unity if the rate of characteristic $x$ ray production caused directly by electron ionization of the targe atoms were proportional to mass concentration. The absorptiol correction factor is so contrived that if certain combined effects o electron scattering and $\mathrm{x}$-ray absorption were the same for unknow! and standard then this factor also would be unity. Similarly, th greater the contribution of fluorescence by characteristic lines, the the further the fluorescence correction factor departs from unity Fluorescence by the continuum is considered by means of anothe factor with similar interpretation. 
The arrangement of these contributions into factors has three disadvantages. First of all, these factors do not contain only those effects that they presume to describe. Each factor includes other contributions. Secondly, if $I_{1}, I_{2}$, and $I_{3}$ are calculated individually, then the factorization creates a mathematical redundancy that increases computational labor. Thirdly, this redundancy tempts us to make approximations in order to simplify the formula. One approximation that suggests itself immediately is to take

$$
\left(1+\frac{I_{3}}{I_{1}+I_{2}}\right) \approx\left(1+\frac{I_{3}}{I_{1}}\right)
$$

This introduces very little error, for $I_{2}$ and $I_{3}$ are generally small, compared to $I_{1}$. Nevertheless, this approximation is not necessary if we use $I=I_{1}+I_{2}+I_{3}$.

Approximations have been made in several derivations of the factor for fluorescence by characteristic lines,

$$
\left(1+\frac{\mathrm{I}_{2}}{\mathrm{I}_{1}}\right)
$$

To illustrate this, it will be necessary to express $I_{1}$ and $I_{2}$ in some detail.

The primary radiation for element A can be written as

$$
\mathrm{I}_{1}=\mathrm{G}_{A} \mathrm{f}_{A}\left(\chi_{A}\right)
$$

where the absorption function $\mathrm{f}(\chi)$ applies to element-A radiation. The factor $G_{A}$ represents the total radiation of element $A$ that is generated throughout the specimen, before any absorption occurs.

It has been shown [1] that the element- $A$ radiation fluoresced by element-B characteristic radiation can be expressed in terms of $f_{B}(\chi)$ as follows

$$
\mathrm{I}_{2}=\mathrm{f}_{B}\left(\chi_{A}\right) \cdot(1 / 2) \mathrm{G}_{B} \mathrm{C}_{A} \omega_{A}(1-1 / \mathrm{J})_{A} \mu_{A B}
$$

$\left\{\left(1 / \chi_{A}\right) \cdot \ln \left(1+\chi_{A} / \mu_{M B}\right)+\int_{\mu_{M B}}^{\infty} \frac{1-\mathrm{f}_{B}(\mathrm{t}) / \mathrm{f}_{B}\left(\chi_{A}\right)}{\mathrm{t}\left(\mathrm{t}-\chi_{A}\right)} \mathrm{dt}\right\}$ 
Simplification of the integral above will be described a little later.

At the moment, consider what happens when the effect of fluorescence is expressed as a factor:

$$
\left(1+\frac{\mathrm{I}_{2}}{\mathrm{I}_{1}}\right)=1+\frac{\mathrm{f}_{B}\left(\chi_{A}\right)}{\mathrm{f}_{A}\left(\chi_{A}\right)} \cdot \frac{1}{2} \frac{\mathrm{G}_{B}}{\mathrm{G}_{A}} \mathrm{C}_{A} \omega_{A}\left(1-\frac{1}{\mathrm{~J}}\right)_{A} \mu_{A B}\{\cdots\}
$$

A natural temptation is to simplify the expression by making the approximation

$$
\frac{f_{B}\left(\chi_{A}\right)}{f_{A}\left(\chi_{A}\right)} \approx 1
$$

In fact, this is precisely one of the approximations made by Castaing in his derivation [2], and made also by several others $[3,4]$ who have developed or modified formulas. It is not obvious whether this approximation always is a safe one to make. Using the Philibert expression for $\mathrm{f}(\chi)$, as modified by Duncumb and Shields [5], one finds that for a $10 \% \mathrm{Cr}-90 \% \mathrm{Ni}$ specimen, analyzed with a probe voltage of $20 \mathrm{kV}$ and a take-off angle of $10^{\circ}$,

$$
\frac{\mathrm{f}_{N i}\left(\chi_{C_{r}}\right)}{\mathrm{f}_{C r}\left(\chi_{C_{r}}\right)}=1.03
$$

Fortunately, though, this $3 \%$ error in the fluorescence intensity contributes only a .3\% error to the total intensity, for this particular case. Perhaps for some other specimen the errors would be greater. At any rate, these errors can be avoided simply by not making the approximation.

The integral that occurs in the complete formula (Eq. 11) can be eliminated easily whenever $\mathrm{f}(\mathrm{t})$ is represented by a rational function (a quotient of two polynomials). For example, one of the approximations of Castaing was to use

$$
\phi(\rho \mathrm{z}) \propto \mathrm{e}^{-\sigma \rho \mathrm{z}}
$$

in part of his derivation [2]. From the definition of $f(\chi)$ (Eqs. 3 and 4), one may calculate

$$
\mathrm{f}(\mathrm{t}) \approx \frac{\sigma}{\mathrm{t}+\sigma}
$$


which permits integration in closed form, leading to

$$
\{\cdots\} \approx \frac{1}{\chi_{A}} \ln \left(1+\frac{\chi_{A}}{\mu_{M B}}\right)+\frac{1}{\sigma} \ln \left(1+\frac{\sigma}{\mu_{M B}}\right)
$$

This result is the same as Castaing's, which originally was obtained through a different mathematical formulation.

Although Castaing's formula is a good compromise between accuracy and mathematical complexity, it would seem reasonable to seek further improvement by taking advantage of the general formula (Eq. 11), and using whatever additional information we have concerning $f(\chi)$. At present, a useful expression is Philibert's [6]

$$
\mathrm{f}_{B}(\chi)=\frac{1+\mathrm{h}}{\left(1+\chi / \sigma_{B}\right)\left[1+\mathrm{h}\left(1+\chi / \sigma_{B}\right)\right]}
$$

This, of course, is a rational function, and permits reduction of the integral in equation (11), leading to

$$
\begin{aligned}
& \{\cdots\}=\frac{1}{\chi_{A}} \ln \left(1+\frac{\chi_{A}}{\mu_{M B}}\right) \\
& +\frac{\mathrm{b}_{2}+\chi_{A}}{\mathrm{~b}_{1}\left(\mathrm{~b}_{2}-\mathrm{b}_{1}\right)} \ln 1+\frac{\mathrm{b}_{1}}{\mu_{M B}}+\frac{\mathrm{b}_{1}+\chi_{A}}{\mathrm{~b}_{2}\left(\mathrm{~b}_{1}-\mathrm{b}_{2}\right)} \ln \left(1+\frac{\mathrm{b}_{2}}{\mu_{M B}}\right)
\end{aligned}
$$

where

$$
\mathrm{b}_{1}=\sigma_{B} \text { and } \mathrm{b}_{2}=\frac{1+\mathrm{h}}{\mathrm{h}} \sigma_{B}
$$

The result is slightly more complicated than Castaing's formula, but would be practically no more time-consuming in computer calculations.

This expression was compared with Castaing's (Eq. 17) for a $10 \% \mathrm{Ti}-90 \% \mathrm{Cr}$ binary analyzed at $30 \mathrm{keV}$ with a take-off angle of $15^{\circ}$. The Philibert $f(\chi)$, as modified by Duncumb and Shields [5] was used. If the same parameters are substituted into equations 17 and 19 , the values differ by $4.1 \%$. A more appropriate comparison is to use the Philibert $\sigma$ [6], as recommended by Reed [4], 
when evaluating equation 17. If the Duncumb-Shields $\sigma$ is used, as before, in equation 19 , then these two expressions differ by $7 \%$. This produces more than $1 \%$ error in the total calculated intensity. Errors of this size might not be particularly serious. The point is that here is one more source of error that now can be avoided.

\section{Conclusion}

The effect of fluorescence by characteristic lines can be expressed in terms of $f(\chi)$, without any need for $\phi(\rho \mathrm{z})$ per se. When $\mathrm{f}(\chi)$ is represented by a rational function, the resulting formula has a form that is convenient for calculations. In particular, Philibert's $f(\chi)$ is well suited, and produces a formula only slightly more complicated than Castaing's. As better expressions for $f(\chi)$ are developed, the formula for fluorescence by characteristic lines can be improved correspondingly.

\section{References}

[1] Criss, John W., "Secondary Fluorescence in Terms of $f(\chi)$ ", Paper No. 9, Second National Conference on Electron Microprobe Analysis, Boston, Mass., June 1967.

[2] Castaing, R., Thesis, University of Paris, 1951; translation by Duwez, P., and Wittry, D. B., WA L 142/59-7, California Institute of Technology, 1955.

[3] Wittry, D. B., Thesis, California Institute of Technology, 1957.

[4] Reed, S. J. B., British Journal of Applied Physics, Vol. 16, 1965, pp. 913-926.

[5] Duncumb, P., and Shields, P. K., The Electron Microprobe, McKinley, T. D. Heinrich, K. F. J., and Wittry, D. B., eds., Wiley, New York, 1966, pp. 284295.

[6] Philibert, J., X-Ray Optics and X-Ray Microanalysis, Pattee, H. H., Cosslett, V. E., and Engstrom, A., eds., Academic Press, New York, 1963, pp. 379. 392. 


\title{
COMPUTATIONAL METHODS FOR X-RAY EMISSION FROM TARGETS EXCITED BY ELECTRONS
}

\author{
D. B. BROWN
}

Electrical Engineering Department, University of Southern California*, Los Angeles, California 90007

\begin{abstract}
This review treats the question, what methods are at our disposal for the theoretical calculation of the production of electron excited $x$-radiation. Three approaches to the problem are described: numerical solution of a Boltzmann equation for electron transport, analytical solution of a Boltzmann equation in terms of moments of the electron distribution, and Monte Carlo calculation of the travel of a representative group of electrons. The differences and relative advantages of the three methods are stressed. Limitations of the theory and of the necessary input parameters to the theory are commented on. Some significant predictions of the theory are compared with experimental work.
\end{abstract}

\section{Introduction}

The aim of this paper is a brief critical survey of the presently available techniques for the calculation of the distribution in depth of primary (electron excited) x-ray production. The approach will be personal and selective rather than objective and exhaustive. First, we analyze three quite different computational approaches which have been tried with some success. Secondly, we consider the common body of physical theory on scattering, energy loss, and ionization phenomena which is used by each of the computational methods. Then we will compare the predictions of theory with various experimental data and attempt a judgment of the status of the theoretical (and experimental) research program. Finally, a few comments on several courses of action which might or should be followed up.

* Presently at Naval Research Laboratory, Washington, D.C. 


\section{Theoretical Discussion}

\section{A. THREE COMPUTATIONAL TECHNIQUES}

We shall be interested in the bombardment of a homogeneous semi-infinite solid target with electrons of perhaps $30 \mathrm{keV}$. Before beginning let us introduce three fairly reasonable simplifying assumptions. First, the impinging electrons will be assumed to lose energy only through collisions with atomic electrons. Second, they will be assumed to be deflected only by two-particle elastic scattering interactions with the field of the atomic nuclei. Third, after an electron has lost a certain energy to the target, the path length it has traveled in the target will be represented by the mean path traveled by electrons while losing this much energy. Each of these simplifications may be somewhat relaxed, but it is the fact that these are a reasonable schematization which gives this problem such simplicity as it possesses. Two additional properties of this physical process can often be used to simplify analysis. They are the facts that scattering is predominantly into small angles, and that after the electrons have been frequently scattered their travel becomes a diffusion process.

The Transport Equation:

The travel of high-energy electrons in solids may be handled with a Boltzmann transport equation. The essence of this treatment is that one may treat the particles not individually but rather with a "distribution function"

$$
\mathrm{f}(\overline{\mathrm{r}}, \overline{\mathrm{v}}, \mathrm{t})
$$

which is the probability of a particle at time $t$ lying within $\mathrm{d}^{3} \mathrm{r}$ around $\bar{r}$ and having a velocity vector lying within $d^{3} v$ around $\bar{v}$. If we assume a one-to-one relation between path traveled, $s$, and velocity, $v$, then the same information will be given by

$$
\mathrm{f}(\overline{\mathrm{r}}, \overline{\mathrm{u}}, \mathrm{s})
$$

where $\bar{u}$ is a unit vector in the direction of motion. The time and the magnitude of the velocity are both specified by the distance the electron has traveled, s. Now, the equation of motion for the distribution function, the transport equation, is

$$
\begin{aligned}
& (\partial / \partial \mathrm{s}) \mathrm{f}(\overline{\mathrm{r}}, \overline{\mathrm{u}}, \mathrm{s})=-\overline{\mathrm{u}} \cdot \nabla_{r} \mathrm{f}(\overline{\mathrm{r}}, \overline{\mathrm{u}}, \mathrm{s})+ \\
& \mathrm{N} \int[\sigma(\alpha, \mathrm{v}) \sin \alpha \mathrm{d} \alpha \mathrm{d} \beta][\mathrm{f}(\overline{\mathrm{r}}, \overline{\mathrm{u}}, \mathrm{s})-\mathrm{f}(\overline{\mathrm{r}}, \overline{\mathrm{u}}, \mathrm{s})]
\end{aligned}
$$


( $\mathrm{N}$ is the number of scatterers (atoms) per $\mathrm{cm}^{3}: \sigma(\alpha, v)$ is the cross section for scattering of an electron of velocity $\mathrm{v}$ through angle $\alpha$; $\sin \alpha \operatorname{d} \alpha \mathrm{d} \beta$ is the solid angle lying between $\alpha$ and $\alpha$ plus $\mathrm{d} \alpha, \beta$ and $\beta$ plus $\mathrm{d} \beta$ where $\beta$ is the azimuthal angle in spherical coordinates; $\overline{\mathrm{u}}^{\prime}$ is some other direction of motion making an angle $\alpha$ with $\overline{\mathrm{u}}$ ). In this equation the first term on the right hand side represents convection or streaming, the first term in the integral represents scattering into the solid angle at $\bar{u}$ and the second term represents the scattering out of it. This very complicated differential-integral equation can be much simplfied by introducing the "small angle approximation". The scattering terms are given a series expansion and, recalling that scattering is predominantly through small angles, we retain only

$$
(\partial / \partial \mathrm{s}) \mathrm{f}(\overline{\mathrm{r}}, \overline{\mathrm{u}}, \mathrm{s})=-\overline{\mathrm{u}} \cdot \nabla_{r} \mathrm{f}(\overline{\mathrm{r}}, \overline{\mathrm{u}}, \mathrm{s})+(1 / \lambda) \nabla_{u}^{2} \mathrm{f}(\overline{\mathrm{r}}, \overline{\mathrm{u}}, \mathrm{s})
$$

where $\lambda$ is the momentum mean free path

$$
(1 / \lambda)=\pi \mathrm{N} \int \sin \alpha \mathrm{d} \alpha \sigma(\alpha, \mathrm{v})(1-\cos \alpha)
$$

Furthermore, we shall consider only the distribution in depth below the specimen surface. This reduces the number of independent variables from seven to only three, viz

$$
\begin{aligned}
& (\partial / \partial \mathrm{s}) \mathrm{f}(\mathrm{x}, \theta, \mathrm{s})=-\cos \theta(\partial / \partial \mathrm{x}) \mathrm{f}(\mathrm{x}, \theta, \mathrm{s})+ \\
& (1 / \lambda)(1 / \sin \theta)(\partial / \partial \theta)[\sin \theta(\partial / \partial \theta) \mathrm{f}(\mathrm{x}, \theta, \mathrm{s})]
\end{aligned}
$$

where $\theta$ is the angle between the direction of motion and the internal normal to the specimen surface. This equation appears much less formidable and can in fact be solved numerically given $\lambda$ as a function of $v$, and $v$ as a function of $s[1,2]$. Knowing $f(x, \theta, s)$ it is not difficult to calculate the distribution in depth of primary $x$-ray production given the probabilities for ionization of the relevant atomic shell. The numerical solution also yields very interesting information on the distribution in energy and angle of electrons backscattered from the specimen.

It is useful to know that after a large number of collisions the transport equation may be well approximated by a diffusion equation. We define the total electron density at a given position

$$
\mathrm{F}(\mathrm{x}, \mathrm{s})=\int_{-\pi}^{+\pi} \mathrm{f}(\mathrm{x}, \theta . \mathrm{s})[2 \pi \sin \theta \mathrm{d} \theta]
$$

and the current density

$$
\mathrm{J}(\mathrm{x}, \mathrm{s})=\int_{-\pi}^{+\pi}[\cos \theta \mathrm{f}(\mathrm{x}, \theta, \mathrm{s})][2 \pi \sin \theta \dot{\mathrm{d}} \theta]
$$


Then when

$$
(1 / J)(\partial J / \partial s)<(1 / \lambda)
$$

it may be shown [3] that

$$
(\partial \mathrm{F} / \partial \mathrm{s})=(\lambda / 6)\left(\partial^{2} \mathrm{~F} / \partial \mathrm{x}^{2}\right)
$$

where $\lambda / 6$ is of course a diffusion coefficient. Note that in this transformation we have eliminated one more independent variable and have obtained a much easier equation to work with.

Moment Methods:

Spencer [4], elaborating on the methods of Lewis [5], has solved the Boltzmann equation in terms of the moments of the electron distribution without using the small angle approximation. Unfortunately this solution is for "scattering media which are homogeneous and without boundaries." Here the transport equation is attacked by an expansion of $\mathrm{f}(\mathrm{x}, \theta, \mathrm{s})$ and $\sigma(\alpha, \mathrm{v})$ in spherical harmonics. These expansions are substituted into the transport equation leading to a linked system of differential equations in terms of the expansion coefficients. These coefficients are

$$
\begin{gathered}
\mathrm{f}_{l}(\mathrm{x}, \mathrm{S})=2 \pi \int_{-1}^{+1} \mathrm{~d}(\cos \theta) \mathrm{P}_{l}(\cos \theta) \mathrm{f}(\mathrm{x}, \theta, \mathrm{s}) \\
\sigma_{l}(\mathrm{v})=2 \pi \int_{-1}^{+1} \mathrm{~d}(\cos \alpha)\left\{1-\mathrm{P}_{l}(\cos \alpha)\right\} \sigma(\alpha, \mathrm{v})
\end{gathered}
$$

where the $\mathrm{P}_{n}(\mathrm{z})$ are Legendre polynomials. This series of equations has not been solved for the $\mathrm{f}_{l}(\mathrm{x}, \mathrm{s})$ but it has been solved for the spatial moments of the expansion coefficients

$$
\mathrm{f}_{l n}(\mathrm{~s})=\int_{-\infty}^{\infty} \mathrm{dx} \mathrm{x^{n }} \mathrm{f}_{/}(\mathrm{x}, \mathrm{s})
$$

It is easy to see, for example, that the mean depth for a given value of $\mathrm{s}$ is

$$
\langle\mathrm{x}(\mathrm{s})\rangle=\mathrm{f}_{01}(\mathrm{~s})
$$

since $\mathrm{P}_{o}(\mathrm{z})=1$. Metchnik and Tomlin [6] have treated $\mathrm{x}$-ray production using a simplified version of Lewis' theory. They use only his mean depth, which may be written

$$
\langle\mathrm{x}(\mathrm{s})\rangle=\mathrm{f}_{01}(\mathrm{~s})=\int_{0}^{\mathrm{s}} \mathrm{ds} \exp \left[-\int_{0}^{s}(2 / \lambda) \mathrm{ds}\right]
$$


where $\lambda$ is again the momentum mean free path (a function of $v$ and therefore of s). As the Metchnik and Tomlin treatment is rather overly simple we shall not consider it further. The Spencer treatment may be useful for our problem in cases where the effect of the boundary at $\mathrm{x}=\mathrm{O}$ can be neglected. Its principal advantage appears to be its avoidance of the small angle approximation. From the point of view of computational difficulty it appears to be at least as troublesome as the other techniques that we shall discuss.

Monte Carlo Methods:

The Monte Carlo technique has a quite different structure from the two just described. It does not deal with a distribution function but rather makes a random sampling of calculated paths of individual electrons. The simulated electron trajectories are generated by using random numbers to determine the new direction and energy of an electron after each scattering event by sampling from the appropriate scattering distribution, and also to determine the distance the electron travels between events. The technique as just sketched is often prohibitively expensive in computer time, and in order to reduce the required amount of computation it is common to abandon the complete description of particle histories. In this approximation the availability of analytical solutions for certain aspects of the multiple scattering problem is utilized. A "condensed" history is sampled by letting the particle carry out a random walk in which each step takes into account the effect of many collisions [7].

For instance, in the treatment of Bishop [8] of our problem the electron trajectory is divided into 25 steps. The electron is assumed to scatter at some random fraction of the step length through an angle selected from a scattering distribution given by GoudsmitSaunderson multiple scattering theory [9]. The latter gives the angular distribution of electrons having traveled a distance $s$ in terms of a series in Legendre polynomials. (We shall give a bit more detail on this theory below.) The physical assumptions used by Bishop in his Monte Carlo calculation [10] are very similar to those used in the numerical solution of the transport equation $[1,2]$. The only clear difference is that the Goudsmit-Saunderson theory does not use the small angle scattering approximation. Differences resulting from this fact would be expected to show up most clearly in the energy distribution of backscattered electrons. This is due to the 
fact that the small angle approximation is expected to underestimate the large angle scattering (5). These differences have not been observed, as will be discussed below. As used in the above examples, the transport equation solution appears to be slightly more efficient than the Monte Carlo solution. This is no doubt partly due to the ability to shift to a diffusion approximation to the transport equation when sufficient scattering has taken place. Unfortunately a comparison of the efficiencies of the two techniques is a bit speculative because of the difficulties of comparing work by different programmers on different computing machines.

\section{B. SINGLE AND MULTIPLE SCATTERING THEORY}

Let us look briefly at the theory of electron scattering available to be introduced into the computational schemes outlined above. A quantum mechanical treatment using the Born approximation (a first order perturbation theory) gives the curious result that the cross section for scattering of an incident electron by the nucleus is given correctly by the classical Rutherford cross section. The Born approximation is better as one goes to high energy electrons, to low atomic number nuclei, and to small scattering angles. Comparison with the more accurate Mott theory [11] indicates that the Rutherford scattering cross section for a scattering angle of $180^{\circ}$ and an electron energy of $50 \mathrm{keV}$ is $20 \%$ high for $\mathrm{Al}$ and $225 \%$ low for $\mathrm{Pb}$. The discrepancies are very much smaller for the predominant small angle scattering. A further problem is that the Rutherford cross section blows up as the scattering angle goes to zero (as the impact parameter goes to infinity). This difficulty can be overcome by taking into account the fact that the Coulomb field of the nucleus is shielded by the atomic electrons at large scattering parameters. This introduces the problem of choosing a reasonable representation of $\mathrm{V}(\mathrm{r})$, the atomic field. For atoms which are not too light one may use the Thomas-Fermi statistical atomic model where

$$
\begin{aligned}
\mathrm{V}(\mathrm{r}) & =-\left(\mathrm{Ze}^{2} / \mathrm{r}\right) \phi(\mathrm{r} / \mu) \\
\mu & =0.885 \mathrm{a}_{0} \mathrm{Z}^{-1 / 3} \\
\mathrm{a}_{0} & =\text { first Bohr radius of } \mathrm{H} \\
& =0.529 \AA
\end{aligned}
$$

The function $\phi(r / \mu)$ has been tabulated. Unfortunately it is known to be inaccurate for large values of $r / \mu$ [12]. This will show up in the small angle (large impact parameter) scattering. The Hartree-Fock field is better for low $\mathrm{Z}$ and large $\mathrm{r}$ but its application can be a 
lengthy procedure. Moreover, neither of these theories take into account the modification in the potential due to solid binding. This too will affect the small angle-scattering. Massey [13] suggests that the following approach may be "as accurate as the neglect of modifications due to the solid justifies, except for the lighter atoms." We approximate the Thomas-Fermi field by

$$
\phi(r / \mu)=\mathrm{e}^{-c r / \mu}
$$

where $\mathrm{c}$ is an empirical constant of order unity. Using the Born approximation this yields (in non-relativistic form)

$$
\begin{gathered}
\sigma(\alpha)=\left(\mathrm{Z}^{2} \mathrm{e}^{4} / 4 \mathrm{~m}^{2} \mathrm{v}^{4}\right)\left[\sin ^{2}(\alpha / 2)+\beta^{2}\right]^{-2} \\
\beta=0.565 \mathrm{Z}^{1 / 3} \mathrm{c}\left(2 \pi \mathrm{e}^{2} / \mathrm{hv}\right)
\end{gathered}
$$

Note that $\beta^{2}$ is a damping constant which prevents $\sigma(\alpha)$ from blowing up as $\alpha$ goes to zero. Note also that this is a conveniently simple form for $\sigma(\alpha)$. In particular it draws from Thomas-Fermi theory the property that one equation applies to all atomic numbers (if they are not too small).

In any treatment of our problem account must be taken of the probability that an electron may scatter more than once. In Eq. 8, containing the small angle approximation, this process is covered by the Laplacian term

$$
(1 / \lambda) \nabla_{u}^{2} \mathrm{f}(\overline{\mathrm{r}}, \overline{\mathrm{u}}, \mathrm{s})
$$

where the cross section for single scattering is contained within $\lambda$. Substitution of Eq. 14 into Eq. 3 yields, neglecting small terms

$$
(1 / \lambda)=\left(\pi \mathrm{NZ}^{2} \mathrm{e}^{4} / \mathrm{m}^{2} \mathrm{v}^{4}\right)\left[\ln \left(1 / \beta^{2}\right)-1\right]
$$

Note that the damping constant is contained in a logarithm. This somewhat reduces the sensitivity of $\lambda$ to a proper choice of the empirical constant $\mathrm{c}$ which appears in $\beta$. One good way of evaluating $c$ is by comparison with a numerical determination of $\lambda$ using a Hartree-Fock potential.

In the Goudsmit-Saunderson multiple scattering theory used by Bishop [9,8] a property of Legendre polynomials is exploited. If $\theta_{1}$ is the deflection after one impact and $\theta$ is the total deflection after $\mathbf{n}$ impacts then

$$
\left\langle\mathrm{P}_{l}(\cos \theta)\right\rangle=\left\langle\mathrm{P}_{l}\left(\cos \theta_{1}\right)\right\rangle^{n}
$$

That is, the average value of any polynomial after $\boldsymbol{n}$ impacts is equal to the $\mathrm{n}$ th power of the average value of that polynomial after one 
impact (providing that the scattering law is cylindrically symmetrical). The Goudsmit-Saunderson work is a predecessor of the Lewis work and is closely related. It concerns itself only with the angular distribution, yielding

$$
\begin{aligned}
\mathrm{F}(\theta, \mathrm{s}) & =\int \mathrm{f}(\overline{\mathrm{r}}, \overline{\mathrm{u}}, \mathrm{s}) \mathrm{dr}=(1 / 2 \pi) \sum_{l=0}^{\infty}(l+1 / 2) \mathrm{P}_{l}(\cos \theta) \\
& \times \exp \left[-\mathrm{N} \int_{0}^{\mathrm{s}} \sigma_{l}(\mathrm{v}) \mathrm{ds}\right]
\end{aligned}
$$

Note again that $\sigma_{l}(\mathrm{v})$ is a generalization of $1 / \lambda$ (compare Eqs. 3 and 9, and Eqs. 11 and 16). Once again this solution is for a medium with no boundaries, and $\theta=0$ corresponds to the initial direction of the electrons. Note finally that the Goudsmit-Saunderson theory retains the details of any chosen single scattering cross section in the various $\sigma_{l}(\mathrm{v})$.

One of the virtues of a numerical solution of a problem is the ease with which one may make a judicious combination of techniques, using each in that portion of the problem for which it is best suited. For example, the following approach has been found useful. First the electrons are allowed to penetrate a very short distance into the target and to assume an angular distribution given by the cross section for single scattering. This avoids the small angle approximation in a region where it is most important. Then the electrons are allowed to proceed as predicted by Eq. 4, the transport equation with small angle approximation. Finally, after considerable scattering has occurred the electrons proceed as predicted by Eq. 7 , the diffusion equation. This scheme seems to strike a nice compromise between rigor and computational efficiency.

\section{ENERGY LOSS AND STRAGGLING}

The assumption of a one-to-one relationship between the energy of an electron and the distance it has traveled in the target is almost always made. In fact it can be shown that electrons must have a distribution of energies which is approximately Gaussian with a half width at half maximum equal to about

$$
\Delta \mathrm{V}=0.126 \sqrt{\mathrm{Z}\left(\mathrm{V}_{o^{-}} \mathrm{V}\right)}
$$

where $\mathrm{V}$ is the mean energy (in $\mathrm{keV}$ ) of the electrons after havine traveled a certain distance and $V_{o}$ is the initial energy $(s=0)$. The effect of energy straggling is difficult to assess. It may be reasonec that straggling is most pronounced after the angular distribution has become nearly isotropic and thus the effect of a distribution of values of $\lambda$ is minimized. The effect of straggling must be mosi 
pronounced in the distribution of electrons at considerable depths below the surface. This is clearly so since electrons which have lost less energy than average can travel farther. This is probably not too important for our problem since $\mathrm{x}$-rays produced at great depths are heavily attenuated. Some rough correction for straggling can be attempted by allowing some fraction of the electrons to travel a bit farther than normal and some fraction to travel a bit less far than normal, but the effect of such a correction on the distribution in depth of $x$-ray production is small.

The usual range-energy relation is

$$
\mathrm{s}=\int_{E}^{E_{0}}(\mathrm{dE} / \mathrm{ds})^{-1} \mathrm{dE}
$$

where the mean rate of energy loss $\mathrm{dE} / \mathrm{ds}$ is taken from Bethe's theory, one simple non-relativistic form of which is

$$
-(\mathrm{dE} / \mathrm{ds})=\left(2 \pi \mathrm{e}^{4} \mathrm{~N} / \mathrm{mv}^{2}\right) \mathrm{Z} \ln \left(\mathrm{m}^{2} \mathrm{v}^{4} / \mathrm{I}^{2}\right)
$$

where I is an empirically determined average ionization energy. Two brief comments on Eq. 19. First, the values to be used for I are still in contention [14]. Moreover, the values for I are determined for energies much greater than the $\mathrm{K}$ shell ionization energies. What is to be done for energies lower than this is not completely clear. This is of interest for the calculation of production of $\mathrm{L}$ lines, but fortunately this is usually of interest for heavy elements where the fraction of $\mathrm{K}$ shell electrons is small. Secondly, experimental checks of the Bethe law $[15,16,17]$ give no clear idea of how good it is. The work of Bichsel [18] implies that the expected accuracy should lie between $1 \%$ and $5 \%$. Now, a comment on Eq. 18 which contains the implicit assumption that

$$
(\mathrm{ds} / \mathrm{dE})=(\mathrm{dE} / \mathrm{ds})^{-1}
$$

Since energy loss is not a continuous process this is not strictly true but an approximation. Spencer and Fano [19] have treated this problem in some detail. A first approximation solution of SpencerFano theory to the same non-relativistic approximation as Eq. 19 is

$$
(\mathrm{ds} / \mathrm{dE})=-\left[\left(2 \pi \mathrm{Ne}^{4} / \mathrm{mv}^{2}\right)\right]^{-1}\left[\ln \left(4 \mathrm{~V}^{2} / \mathrm{I}^{2}\right)+\mathrm{l}-\ln \left[\mathrm{V} /\left(\mathrm{V}_{0}-\mathrm{V}\right)\right]\right.
$$

Note that this equation tends to blow up as $\mathrm{V}$ approaches $\mathrm{V}_{0}$. This correctly indicates a reduction in the rate of energy loss in the vicinity of $\mathrm{V}_{o}$. This results from the fact that energy losses greater than $\mathrm{V}_{0}-\mathrm{V}$ are not possible. A mean range determined from a version 
of Spencer-Fano theory has been applied to our problem [2]. The effects on the distribution in depth of x-ray production are not striking. The maximum in the energy distribution of back scattered electrons does shift to higher energies, improving agreement with experiment.

D. IONIZATION CROSS SECTIONS, COSTER-KRONIG, ETC.

To calculate the distribution of $\mathrm{x}$-ray production from the electron distribution function of course requires information on ionization probabilities. The fact that in microprobe work one is almost always using ratios of intensities serves to reduce the sensitivity of the problem to the details of these probabilities. This is fortunate, since both the experimental and theoretical work in this area are in an unsatisfactory state. A combination of theoretical and experimental considerations suggest that the cross section for $\mathrm{K}$ shell ionization may be roughly represented by the form

$$
\mathrm{Q}_{K} \text { varies as }\left(1 / \mathrm{V}_{K^{2}}\right)\left(\mathrm{V}_{K} / \mathrm{V}\right)^{n} \ln \left(\mathrm{V} / \mathrm{V}_{K}\right)
$$

where $\mathrm{n}$ is a constant of the order of one. The constant of proportionality is not necessary for the determination of a ratio of intensities. The situation for $\mathrm{L}$ lines is less satisfactory. For lack of a better solution it is usually assumed that $\mathrm{Q}_{L}$, has the same form as $\mathrm{Q}_{\kappa}$. Furthermore, in the case, for instance, of $\mathrm{L}_{I \prime \prime}$ ionization one really should account for the probability that an $\mathrm{L}_{l}$ ionization may make a non-radiative transition to an $\mathrm{L}_{I I}$ ionization. To do this one needs the Coster-Kronig coefficients giving the transition probabilities (not well known) and also the ratio $\mathrm{Q}_{L, I H} / \mathrm{Q}_{L_{L} I}$ (not well known). Once again, the effect of these uncertainties is somewhat reduced by taking intensity ratios. Finally, if one wishes to calculate the absolute intensity of $x$-ray production one needs to know the constant of proportionality for Eq. 21 and to know the fluorescence yield factors. These latter are not well known for low atomic number.

\section{E. IONIZATION DUE TO FLUORESCENCE}

Secondary ionization may occur as a result of fluorescence by the continuum or by sufficiently energetic characteristic lines. The latter is usually of importance only when the exciting line is close to the ionization energy of the excited line. This effect has been treated well enough for our purposes by Reed [20]. Fluorescence due to the continuum can also be treated approximately. In this case the 
mathematics may become very tedious, especially when there are many absorption edges between the ionization energy and the short wavelength limit. Nevertheless, the key physical assumptions involved in this treatment may be stated concisely. All of the exciting radiation is assumed to be produced at the surface and to travel in all directions with equal probability. Thus half of the intensity is immediately lost from the target. The number of continuous quanta per electron located between $\lambda$ and $\lambda$ plus $d \lambda$ is assumed to follow a law like

$$
\mathrm{dN}_{c}(\lambda)=34.2 \times 10^{-6} \mathrm{Z} \frac{\lambda-\lambda_{S W L}}{\lambda^{2} \lambda_{S W L}} \mathrm{~d} \lambda
$$

where $\lambda_{S W_{L}}$ is the short wavelength limit of the continuous spectrum.

\section{Experimental Justification}

We have discussed some of the possibilities and problems basic to the calculation of the distribution in depth of $x$-ray production. Now let us see if we can make a judgment as to the success of such calculations by comparing predictions with experimental results.

In Figure 1, the predictions of the transport equation program (TEP) [2] are compared with the results of Castaing and Descamps' tracer experiment [21]. The agreement is encouraging. The differences for $\mathrm{Au}$ ( $\mathrm{Bi}$ tracer) at low mass-depths are thought to result from the use of a somewhat too thick tracer layer in the experiment. It is not really clear why the calculated $\mathrm{x}$-ray production lies as far as it does above the measured in the case of $\mathrm{Cu}$ ( $\mathrm{Zn}$ tracer) at large mass-depth. Figure 2 compares the tracer experiments of Castaing and Hénoc with the predictions of the TEP and of Bishop's Monte Carlo program [22]. Here also the agreement is sufficiently good that it is difficult to decide in what ways the theory may be superior or inferior to the experimental data. In Figure 3, we look at the energy distribution of backscattered electrons. Here the TEP and Monte Carlo predictions are compared with the measurements of Kulenkampff and Spyra and of Bishop [23,8]. The Kulenkampff and Spyra data have been normalized so that the total backscattered fraction agrees with that measured by Bishop [8]. It is interesting that the TEP and Monte Carlo results are very similar. This would seem to indicate that the introduction of the smail angle approximation 


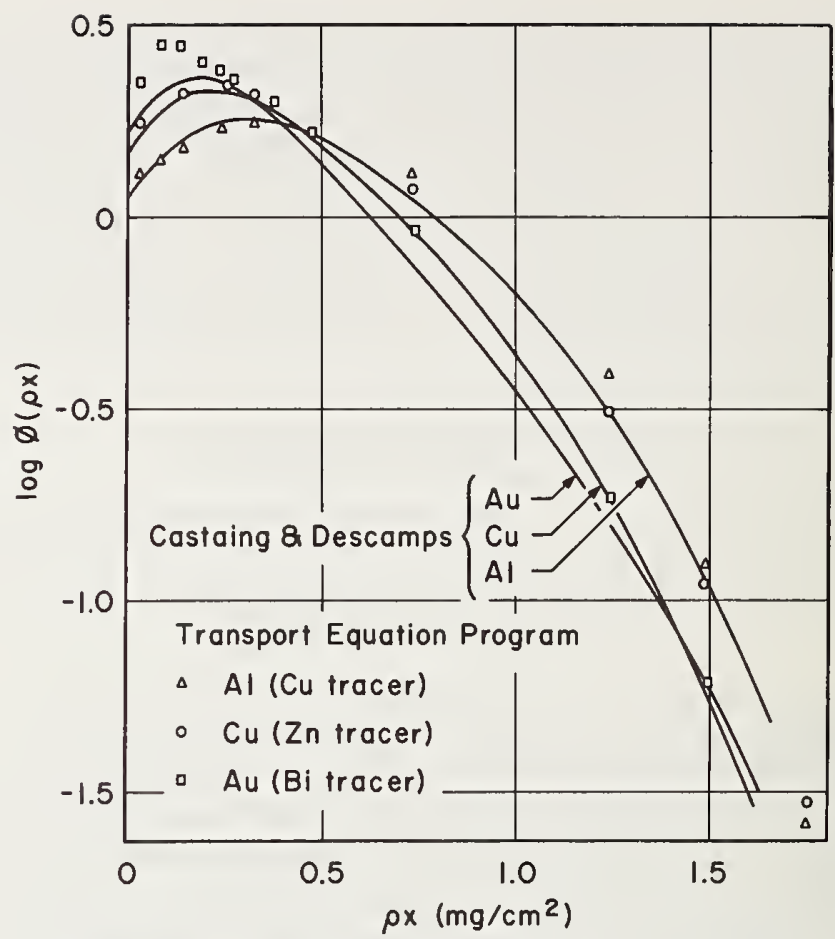

Figure 1. Distribution in depth of primary $x$-ray production. Initial electron energy, $29 \mathrm{keV}$. Normal incidence.

into the transport equation is not serious. Figure 4 compares the predictions of the TEP of the absolute intensity of emerging $\mathrm{x}$-radiation from $\mathrm{Al}$ at a takeoff angle of $15.5^{\circ}$. The experimental data have been previously reported [24] but the voltage has been recalibrated and the Heinrich absorption coefficient [25] has been used. The agreement is surprisingly good since the fluorescence yield factor for $\mathrm{Al}$ is uncertain (we used .0267). In Figure 5, we show the calibration curve ( $\mathbf{I}_{\text {alloy }} / \mathbf{I}_{\text {pure }}$ versus weight fraction) for $\mathrm{Ni} \mathrm{K}_{\alpha}$ radiation in the $\mathrm{Fe}-\mathrm{Ni}$ system. Here the predictions of the TEP are compared with the measurements of Goldstein [26] and with calculations using the absorption corrections of Green [27] and Castaing and Descamps [21]. The surprising thing here is the degree of disagreement between Greer: and Castaing and Descamps. The takeoff angle was $15.5^{\circ}$. Figure $\epsilon$ makes a similar comparison with Ziebold's measurements for the 


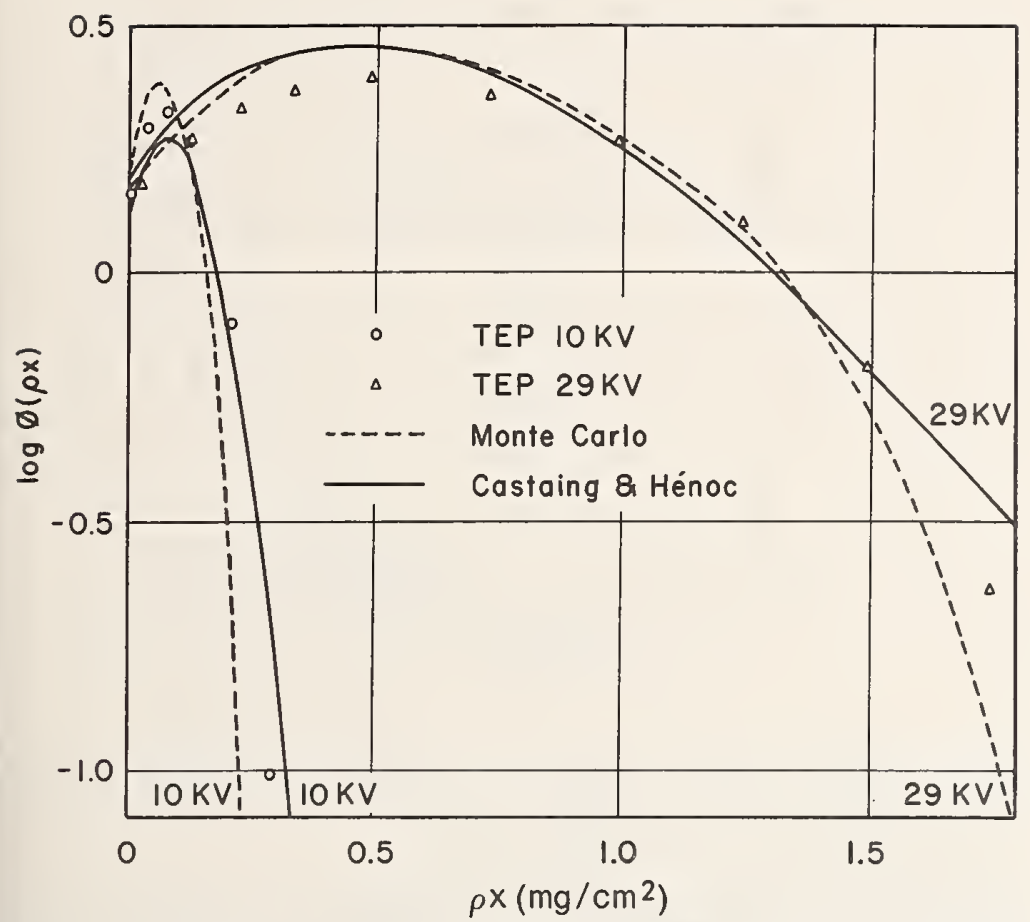

Figure 2. Distribution in depth of primary $\mathrm{x}$-ray production for $\mathrm{Al}$ with a tracer of $\mathrm{Mg}$. Normal incidence.

$\mathrm{Au} \mathrm{L}_{\alpha}$ line in $\mathrm{Cu}-\mathrm{Au}$ alloys [28]. Here we found it necessary to make a correction for secondary ionization due to the continuum. It is calculated that at this takeoff angle $\left(52.5^{\circ}\right) 15.3 \%$ of the measured radiation is due to secondary ionization in the case of pure $\mathrm{Au}$.

The previous comments have indicated the need for more precise experimental work. From the point of view of judgment and improvement of the theoretical work, we are most in need of better data on the energy distribution of backscattered electrons and on the distribution in depth of $\mathrm{x}$-ray production. Also, as has been indicated, the data on ionization probabilities are definitely in need of work.

\section{Conclusions}

\section{A. ANALYSIS OF THE THEORY}

In our judgment the major weakness of the theory at present lies in somewhat questionable schematization of the scattering process. 


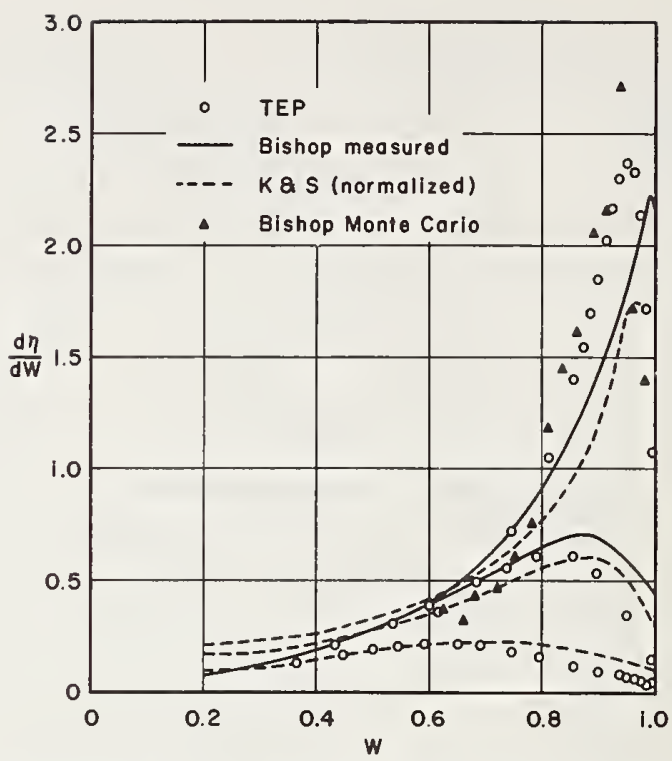

Figure 3. Energy distribution of backscattered electrons. $W$ is $V / V_{o}$. The curves are, in ascending order, for $\mathrm{Al}, \mathrm{Cu}$, and $\mathrm{Au}$ (with the exception of the Kulenkampff and Spyra data where the upper curve is for $\mathrm{Pt}$ ).

Some improvement might be expected from the introduction of a more realistic atomic potential and from correction for the Born approximation and the small angle approximation. These modifications do not appear to be easy. Two possibilities come to mind. First, one can imagine using a Thomas-Fermi-like potential which took better cognizance of the presence of the atom in a solid. Secondly, it might be possible to introduce the Spencer moment method during the early stages of electron travel where the limitation of infinite scattering media is of less importance. This would allow a more exact use of the single scattering cross section during a critical region. A generalization of the theory which would be most interesting would be to determine the three dimensiona distribution rather than just the distribution in depth. This greatly complicates the problem. In the case of the transport equation on goes from three to seven independent variables. Further, it would be interesting to remove the restriction to homogeneous scatterin media. No doubt this could be handled, but the computational effor appears excessive at the moment. 


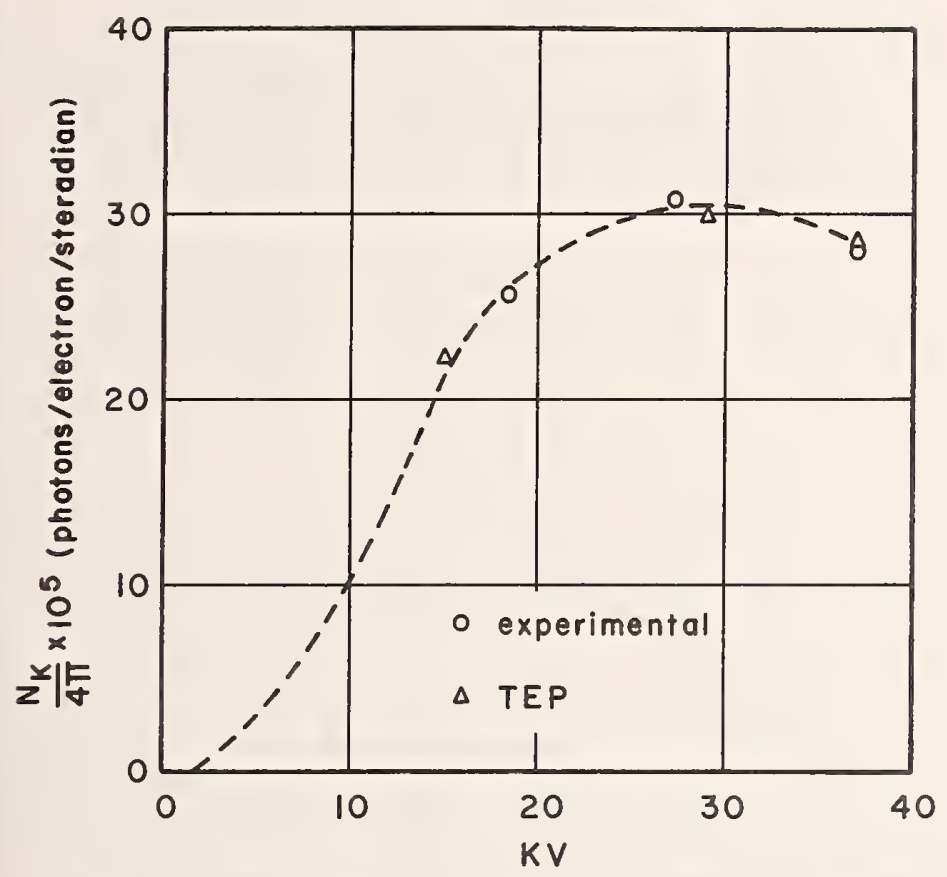

Figure 4. Variation with voltage of $\mathrm{x}$-ray intensity emerging from an Al target at a takeoff angle of $15.5^{\circ}$. Absorption coefficient from Heinrich. Fluorescence yield factor, 0.0267 .

\section{B. COMMENT ON APPLICATION OF THE THEORY}

The art of making corrections for quantitative analysis with the microprobe (empirical calibrations aside) has become polarized around the absorption, fluorescence, and "atomic number" corrections. This division has proven useful, but it is perhaps well to remind ourselves again that it is somewhat artificial. For instance, in the calculations leading to the TEP results in Figures 5 and 6 , the atomic number and absorption correction factors did not enter explicitly. The calculation proceeded directly from an integration over the distribution in depth of primary radiation. Ideally, the easy availability of reliable computer calculations of the x-ray distribution will render these concepts excess baggage. It is not yet clear that we have reached this state. 


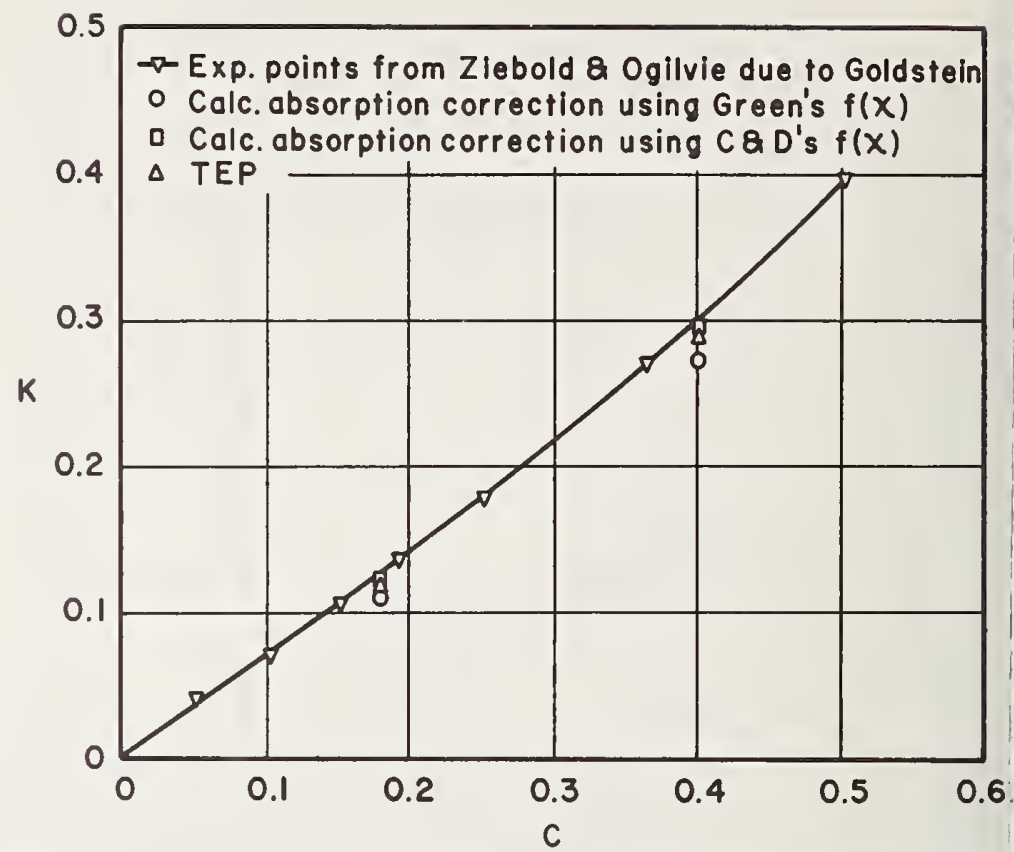

Figure 5. Calibration curve for $\mathrm{Ni}_{\alpha}$ radiation in $\mathrm{Fe}-\mathrm{Ni}$ alloys. $\mathrm{C}$ is the weight fraction of $\mathrm{Ni} . \mathrm{K}$ is the intensity ratio $\mathrm{I}_{\text {at }} / \mathrm{I}_{\mathrm{y}} / \mathrm{I}_{\text {pure }}$. Incident electron energy, $30 \mathrm{keV}$. Takeoff angle, $15.5^{\circ}$.

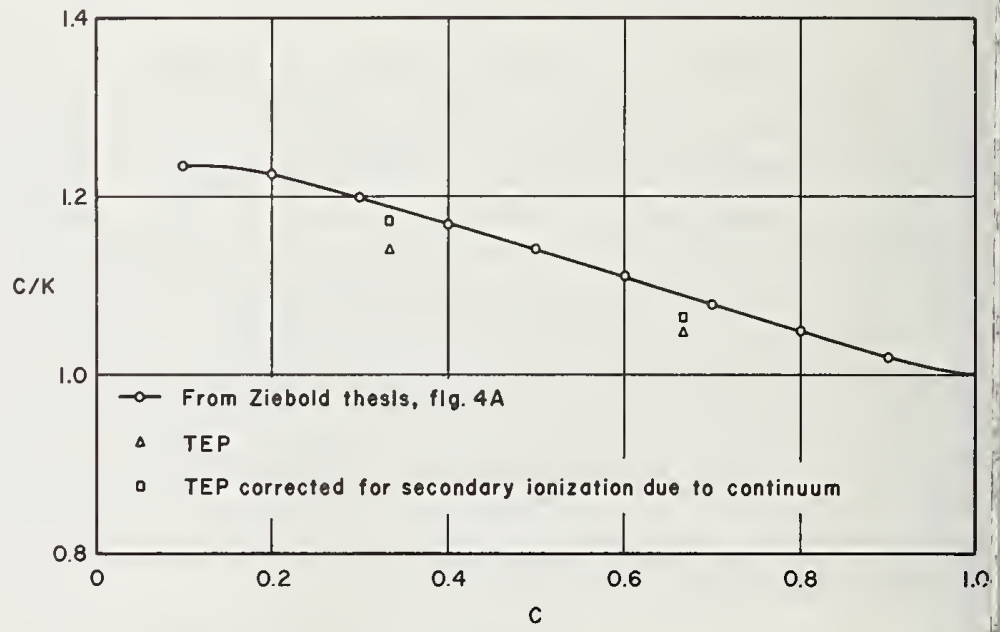

Figure 6. Calibration curve for $\mathrm{Au} \mathrm{L}_{\alpha}$ radiation in $\mathrm{Cu}-\mathrm{Au}$ alloys. $\mathrm{C}$ is the weigh fraction of $\mathrm{Au} . \mathrm{K}$ is the intensity ratio $\mathrm{I}_{\text {alloy }} / \mathrm{I}_{\text {pure }}$. Incident electron energy, $30 \mathrm{keV}$ Takeoff angle, $52.5^{\circ}$. 


\section{References}

[1] Brown, D. B., and Ogilvie, R. E., J. Appl. Phys. 37, 4429 (1966).

[2] Brown, D. B, and Wittry, D. B., presented at the Second National Conference on Electron Microprobe Analysis, Boston, 1967.

[3] Bethe, H. A., Rose, M. E., and Smith, L. P., Proc. Am. Phil. Soc. 78. 573 (1938).

[4] Spencer, L. V., Phys. Rev. 98. 1597 (1955).

[5] Lewis, H. W., Phys. Rev. 78. 526 (1950).

[6] Metchnik, V., and Tomlin, S. G., Proc. Phys. Soc. 81, 956 (1963).

[7] Berger, M. J., Methods in Computational Physics. Vol. 1 (Academic Press, New York, 1963), p. 135.

[8] Bishop, H. E., X-Ray Optics and Microanalysis, (Hermann, Paris, 1966) p. 112.

[9] Goudsmit, S., and Saunderson, J. L., Phys. Rev. 57, 24 (1940).

10] For a detailed treatment of this see H. E. Bishop, Ph.D. Thesis, University of Cambridge, 1966. See also Ref. 8.

11] Reviewed by R. D. Birkhoff, Encyclopedia of Physics Vol. XXXIV (SpringerVerlag, Berlin, 1958), p. 53.

[12] Mott, N. F., and Massey, H. S. W., The Theory of Atomic Collisions, 3rd ed. (Oxford U. Press, London, 1965), p. 462.

[13] Massey, H. S. W., Advances in Electronics, Vol. IV (Academic Press, New York, 1952), p. 1.

[14] For a recent discussion of this see the paper by J. E. Turner, Studies in Penetration of Charged Particles in Matter, Nuclear Science Series Report Number 39, NAS-NRC Publication 1133 (available from the National Academy of Sciences), p. 99.

[i5] Feldman, C., Phys. Rev. 117, 455 (1960).

[16] Holliday, J. E., and Sternglass, E. J., J. Appl. Phys. 30. 1428 (1959).

[17] Cosslett, V. E., and Thomas, R. N., Brit. J. Appl. Phys. 15. 1283 (1964).

[18] Bichsel, H., NSS Report 39 (see Ref. 14), p. 17. The reader is cautioned that this work refers to heavy particles of about the same velocity, but therefore of much higher energy than the electrons of interest to this paper.

[19] Spencer, L. V., and Fano, U., Phys. Rev. 93. 1172 (1954).

[20] Reed, S. J. B., Brit. J. Appl. Phys. 16.913 (1965).

[21] Castaing, R., and Descamps, J., J. Phys. Radium 16. 304 (1955): and Castaing, R., Advances in Electronics and Electron Physics, Vol. XIII (Academic Press, New York, 1960), p. 317.

[22] Castaing. R. and Hénoc, J., X-Ray Optics and Microanalysis, (Hermann, Paris, 1966) p. 120.

[23] Kulenkampff, H., and Spyra, W., Z. Phys. 137,416 (1954).

[24] Brown, D. B., and Ogilvie, R. E., J. Appl. Phys. 35, 309 (1964).

[25] Heinrich, K. F. J., The Electron Microprobe (Wiley, New York, 1966), p. 296.

[26] This data due to J. I. Goldstein was taken from T. O. Ziebold and R. E. Ogilvie, Anal. Chem. 35, 621 (1963).

[27] Green, M., X-Ray Optics and X-Ray Microanalysis (Academic Press, New York, 1963), p. 361.

[28] Ziebold, T. O., Ph.D. Thesis, Massachusetts Institute of Technology, 1965. 



\title{
THE CHOICE OF MODELS FOR ELECTRON SCATTERING AND DECELERATION FOR ELECTRON PROBE MICROANALYSIS
}

\author{
T. MULVEY
}

Department of Physics, The University of Aston in Birmingham, England

\section{Abstract}

The general physical picture of the interaction of the electron beam with the specimen in the x-ray microanalyzer is clear. Nevertheless, many of the numerical results yielded by even the most successful theoretical calculations fall outside the range of experimental error. An improvement in this position can be attained in principle but only at the expense of long and tedious calculations. However, if one is prepared to limit the field of application of a model on an empirical basis or introduce plausible assumptions, surprisingly accurate quantitative analysis can be performed in practice. The present paper reviews various models of this type, and makes some suggestions for further work.

\section{Introduction}

Electron probe microanalysis is now a commonly accepted tool in quantitative microanalysis. Many difficult technical and instrumental problems have been overcome in recent years, so that a remarkable range of specimens can now be examined. For many purposes an approximate idea of the concentration of an unknown element is adequate, but eventually all electron probe users are faced with the problem of providing a quantitative answer, to a relative accuracy of perhaps one or two percent. It is at this level that the real difficulties begin. There are many reasons for this in practice.

\section{Discussion}

\section{A. INSTRUMENTAL ERRORS}

Among these difficulties may be mentioned instrumental shortcomings such as electrical and mechanical drifts and instabilities. Field tests on groups of instruments made by the Midlands probe users group suggest that errors from these causes of 
up to $5 \%$ are not uncommon in day-to-day working, but can be overcome with time and care. Other errors can arise from errors in the data needed for applying various corrections. An obvious example is that of mass absorption coefficients in the soft $x$-ray region. Errors of $100 \%$ in this region have been suspected at times when corrections are being applied to the measured carbon content of uranium carbide, for example.

\section{B. GENERAL CONSIDERATIONS ABOUT MODELS}

The present contribution is not concerned with errors of this type as they contribute only incidentally to error in quantitative analysis. However, there are certain experimental errors which are more insidious and which may influence profoundly the performance of a particular model which forms the basis of the correction procedure For example, the fraction $\eta$ of primary electrons backscattered from a pure element is an important parameter in any empirical method o quantitative correction. In some models, experimentally determined values of backscattering coefficient are built into the method. The accurate measurement of this simple ratio is surprisingly difficult ts carry out in practice. The two most recent and carefu determinations by Philibert and Weinryb (1962) [1] and by Bishor (1966) [2] lead to coefficients that differ by more than 10 percen for heavy elements. A similar situation arises in the more comple) experimental measurement of the distribution as a function o energy of the backscattered electrons. For example, the widely user experimental results of Kulenkampff and Spyra (1954) [3] canno be reconciled quantitatively with those of Bishop (1966) [2] Nevertheless, both sets of data have been successfully put to use i various correction procedures.

\section{IDEAL REQUIREMENTS OF A MODEL}

Ideally this state of affairs should not arise. A genuine theory $c$ electron probe analysis should be based on more fundaments considerations such as the electron scattering and x-ray emittin properties of atoms in metals. Unfortunately it must be admitte that we are not yet in possession of a comprehensive theory : electron interaction with metals. A quotation from Professor S. F Edwards (1967) [4] in a recent article entitled "How do electror really behave in a metal?" runs as follows: "In conclusion I woul say, if I were a pessimist, that metal theory has passed into the sam state of knowledge that classical liquid theory entered many year: 
ago. The picture is there but the numbers are not. If an optimist, I would say that our knowledge is so good that it is really up to theorists to show why it all works (notwithstanding the comments above) as well as it does."

In many ways this statement could serve as a summary of the closely related subject of the present state of the theory of electron probe analysis. It is still possible to take on logical grounds either an optimistic or a pessimistic view of the possibility of deriving all the corrections for quantitative analysis entirely from basic principles. In the meantime samples for analysis are accumulating around the instruments, and quantitative results must be declared with confidence.

\section{TWO APPROACHES TO CORRECTION PROCEDURES}

It is not surprising therefore that two approaches to the subject may be discerned which will certainly influence the choice of model and which are inspired by (1) the need to obtain at all costs the correct result in the quickest and most certain manner possible in the knowledge that considerable liberties have been taken in the process and (2) the ultimate necessity of understanding in detail the underlying physical principles of electron probe analysis so that new situations can be tackled with confidence. In the limit the first approach calls for the production of a close range of comparison samples thereby eliminating the need for any corrections. It is of course difficult to make the range of standards. In the limit the second approach substitutes for the close standard merely the name of the pure standard element. Most correction methods at present in use rely more or less heavily on calibration procedures inasmuch as they contain adjustable constants whose value is determined from experimental measurements on test alloys. For this reason the fact that a particular model or correction procedure leads to the correct analytical result, although extremely consoling, does not necessarily constitute a fundamental advance and may even be invalidated by the arrival of more accurate data. It is therefore useful to analyze empirical methods, successful or otherwise, with a view to discovering their useful area of application.

\section{E. PHYSICAL PICTURES AND QUANTITATIVE THEORIES}

In discussing theories of electron probe analysis it should be recognized that the real difficulty is to obtain a detailed numerical theory to account for the strong interaction of the primary electrons 
with the atomic nucleus and its associated strongly bound electrons of comparable energy in the inner shells. It is not surprising that the greatest progress in the quantitative theory has been in the area of weak interaction such as occurs in secondary fluorescence of the specimen by characteristic or continuous x-rays. In his original derivation of the theory Castaing (1950) [5] preferred to deduce an approximate theory using the experimentally inspired laws of electron retardation of Williams and of Webster. These have the great advantage of leading to the correct answer (to a first approximation), as revealed by subsequent experiment, whilst avoiding several awkward physical problems such as the precise nature of the energy loss process and scattering of electrons in the target.

The physical basis of electron probe analysis put forward by Castaing and Descamps (1955) [6] was more solidly based relying as it did on the experimentally determined distributions of generated $x$-ray intensity with depth by the tracer method. The generalizatior of these results led to a satisfying physical picture of the physica processes that are at work. Nevertheless it should be emphasizec that a correct physical picture is not synonymous with a detailec quantitative theory. For example, a knowledge of the electror density distribution on pure metals does not make it immediately obvious how to analyze an alloy. In fact the labor of calculating accurate electron distributions in pure elements alone is considerable.

Thus the early controversies about the existence or not of ar "atomic number effect" could not be resolved simply by inspecting these experimental distributions. It is always possible of course tha refinements of technique may make it feasible to obtain an adequate number of electron distributions in alloys but at the moment thi approach does not seem to be yielding sufficient data to eliminate the need for accurate calculations.

Another approach might be to measure the intensity o characteristic radiation from a sample as a function of take-off angle Knowing the mass absorption coefficient of the material the required distribution could be found. Because of purely mechanica difficulties and lack of precision in mass absorption data thi experiment cannot be performed to the necessary accuracy. In fact by means of such experiments one can quickly realize that unde typical operating conditions $\mathrm{f}(\chi)$ curves $(\chi=(\mu / \rho) \operatorname{cosec} \theta)$ are no 
very sensitive to the precise form of the electron distribution with depth. Several authors have of course taken full advantage of this fact when calculating $\mathrm{f}(\chi)$ curves from comparatively crude electron models. None of the present models, including the most Monte Carlo methods, is equally accurate for all values of $\chi$ or for all elements. This suggests that, as more accurate $(\mu / \rho)$ values become available it may be possible to refine the experimental technique to the point where it might provide useful information about the electron distribution with depth especially for soft radiations.

\section{F. THE NEED FOR A VARIETY OF MODELS}

Since it has not yet proved possible to consider all corrections simultaneously either experimentally or theoretically, it is necessary to consider them piecemeal. Consider the intensity ratio $\mathbb{I}^{A} / \mathbb{I}[\mathrm{A}]$ measured from sample which contains a weight fraction $C_{A}$ of element A.

$$
\frac{I^{A}}{I[A]}=\frac{C_{A} f_{s}(X) R_{s}}{f_{A}(X) R_{A}} \frac{\int_{E_{o}}^{E_{K}} \frac{Q_{K}(E)}{(d E / d s)_{s}} d E}{\int_{E_{o}}^{E_{K} \frac{Q_{K}(E)}{(d E / d s)_{A}} d E}}
$$

Here $\mathrm{f}(\chi)_{s}$ refers to the sample and $\mathrm{f}(\chi)_{A}$ to the pure standard. $\mathrm{Q}_{h}(\mathrm{E})$ is the ionization cross-section for $\mathrm{K}$ (or $\mathrm{L}, \mathrm{M}$, $\mathrm{N}$, etc.) radiation $(\mathrm{dE} / \mathrm{ds})$ is the electron "stopping power", $\mathbf{R}_{s}$ is a backscattering factor that takes care of the fact that some electrons leave the material with an energy greater than the critical excitation potential $E_{k}$. $E_{o}$ is the initial potential of the electron. Here s refers to the mass/unit area (density $\mathrm{x}$ distance) traversed by the electron along the trajectory.

It should be noted that it is not necessary or even desirable to use the same model for each factor. For example, if the value of $f(\chi)$ is greater than 0.2 the quickest procedure for adequate accuracy is usually the Philibert (1962) [7] expression.

$$
\frac{1}{\mathrm{f}(\chi)}=\left(1+\frac{\chi}{\sigma}\right)\left(1+\frac{\mathrm{h}}{(1+\mathrm{h})} \cdot \frac{\chi}{\sigma}\right)
$$


with $\sigma$ given by Duncumb and Shields (1964) [8] as

$$
\sigma=\frac{2.39 \times 10^{5}}{\mathrm{E}_{\mathrm{o}}{ }^{1.5}-\mathrm{E}_{\mathrm{c}}{ }^{1.5}} \text { and } \mathrm{h}=\frac{1.2 \mathrm{~A}}{\mathrm{Z}^{2}}
$$

It should be mentioned that this expression is based on a mode that assumes that the ionization cross section $\mathrm{Q}_{K}$ does not vary wit] kilovoltage and is therefore likely to be inaccurate where the over voltage ratio $\mathrm{E} / \mathrm{E}_{\kappa}$ and mass absorption coefficients are high e.g. i) light element analysis. In such a case a model which employs a mor exact value for $\mathrm{Q}_{k}$ but is perhaps less satisfactory in other respects is likely to be more accurate.

In many practical cases however, equation (1) can be written

$$
\frac{I^{A}}{I[A]}=C_{A} \frac{f_{S}(X)}{f_{A}(X)} \cdot \frac{R_{S}}{R_{A}} \frac{\bar{S}_{A}}{\bar{S}_{S}}
$$

where $\bar{S}$ represents a suitably averaged stopping power in the targe or pure element. Poole and Thomas (1964) [9] have taken fu advantage of this simplification in their method, which in its vali range of application does not require a knowledge of $\mathrm{Q}_{k}$. It may $\mathrm{b}$ remarked here that the $R$ values assumed by these authors do no agree with those recently determined by Dérian (1966) [10] so tha some readjustment of the other parameters may be needed to tak this into account. The general result is that the atomic number effec tends to be overestimated by Poole and Thomas. The chief factor to be considered in quantitative analysis then are the stopping powe $\mathrm{dE} / \mathrm{ds}$ and the backscattering coefficient $\mathrm{R}$. These factors vary wit atomic number in such a way as to produce an enhancement of th $\mathrm{x}$-ray intensity from the light element in a heavy matrix and depletion of that from a heavy element. The effect is noticeable fo elements of widely different atomic number but can be observe (with some difficulty) in the copper-nickel system. Accurat measurements in this system might well throw some light on th atomic number effect. Experimentally it is difficult to isolate thi effect since even a small uncertainty $( \pm 5 \%)$ in the relevant mas absorption coefficients is often sufficient to blur it out. For thi reason many workers in the field have expressed profoun scepticism even as to its existence. There is little doubt, howevel that the high intensity ratios observed from low concentrations 0 light elements in heavy matrices are principally due to this cause. $f$ 
calculation carried out in 1963 by Archard and the author (unpublished) showed that useful enhancements of several hundred percent for the carbon content of uranium carbide could be expected at accelerating voltages of $5 \mathrm{kV}$ and take-off angles in the $30-45^{\circ}$ region.

\section{G. THE RELATION BETWEEN STOPPING POWER AND BACKSCATTERING}

The backscattering of electrons is closely connected with stopping power. In fact, if the trajectory of the electron is known a separate calculation of backscattering does not arise since the integration of equation (1) can be stopped at the point where the electron leaves the target. This method is ideal but places great reliance on knowing the trajectory. In the target primary electrons are scattered elastically by the (screened) nucleus and inelastically (by the atomic electrons). Clearly these processes are complicated and the loss of energy at each inelastic collision is difficult to calculate. It is however, possible to calculate a mean free path between elastic and inelastic collisions, using methods developed for electron microscopy and discussed by Halliday (1962) [11]. Elastic differential cross-sections can be calculated on the basis of Wentzel's (1927) [12] model which takes some account of the screening effects of the atomic electrons in the nucleus. Inelastic ross-screening effects can be calculated by the method of Lenz 1954) $[13]$.

To give an idea of orders to magnitude: at $30 \mathrm{kV}$ the total mean ree path (inelastic and elastic events) in gold is about $30 \AA$ with a nost probable angle of $5 \times 10^{-2}$ radian for elastic scattering and $\times 10^{-4}$ radian for inelastic scattering. In carbon the corresponding igures would be $80 \AA$ for the mean free path, $3 \times 10^{-2}$ radian for lastic and $1.6 \times 10^{-4}$ radian for inelastic scattering. In gold, elastic cattering would be about two and a quarter times as frequent as pelastic scattering, whereas in carbon, inelastic scattering would e over four times as frequent as elastic scattering.

A model of this general type should be capable, in principle, of ielding valuable information in both electron microscopy and probe halysis. A difficulty arises, however, in the lack of detailed formation about the relation between inelastic scattering and hergy losses. In practice it has normally proved necessary to resort the Bethe (1930) [14] formula which replaces the actual discrete sses by a continuous mean loss. 
As a matter of interest, a somewhat simplified Monte Carlo programme by Lauria (unpublished) based on the foregoing treatment has indicated that the above approach can give usefu results but not of such a degree of accuracy as to justify the prohibitive computing time required.

H. THE BETHE CONTINUOUS ENERGY LOSS RELATION

According to Bethe (1930) [14] the mean energy loss may be written

$$
\frac{d(e \vec{V})}{d(\rho x)}=\frac{d(e \bar{V})}{d s}=-2 \pi \frac{N_{A} Z^{4}}{e V} \ln \frac{2 e \bar{V}}{J}
$$

where $N_{A}$ is Avogadro's number, $Z$ the atomic number and $\bar{\nabla}$ th energy of the electron, and $\mathbf{J}$ is the mean ionization energy of th atom. Although there are many approximate versions of this las which are simpler for hand calculations, most of these achiev simplicity by obscuring the more fundamental constants. It $\mathrm{i}$ therefore worthwhile to examine Bethe's Law in more detail.

An alternative treatment (Bethe 1933) [15] leads to a slightl different logarithmic term in equation (5) namely

$$
\ln \left(\frac{\varepsilon}{2}\right)^{1 / 2}\left(\frac{\mathrm{eV}}{\mathrm{J}}\right) \text { where } \varepsilon=2.718,
$$

the base of natural logarithms. For an excellent discussion of thes and other related topics see Bishop (1966) [2]. Probably the mo difficult term to calculate in equation (5) is the mean ionizatid energy $\mathbf{J}$. Unfortunately in electron probe analysis the range energies of the electrons is large and the energies comparable wi the binding energy of the atomic electrons themselves; this mak calculation difficult. Until recently it was generally assumed calculation in electron microscopy and probe analysis that expression of the form $\mathrm{J} \Omega 11.5 \mathrm{Z}$ electron volts adequate described the situation, especially as the term occurred as logarithm.

\section{REVISED VALUES OF $\mathbf{J}$ ?}

Recently this view has been challenged by Duncumb and da $\mathrm{Ca}$ (1967) [16] following a systematic analysis of many w authenticated analyses of microprobe specimens of knoy composition. By a process of elimination of various errors 
correction procedure, these authors have found it necessary to postulate a very different relation between ionization energy and atomic number from that given above. This suggests that more intensive study should be made into this question.

Another aspect of the Bethe equation that has been emphasized by Bishop (1966) [2] in his Monte Carlo calculations, is that the statistical straggling of electrons in the specimen is ignored in the Bethe equation and that this could cause errors. This effect is also thought to be a principal source of error in Green's method using experimental scattering data from thin films to build up a Monte Carlo calculation of a solid target, using the Bethe equation to calculate the energy loss in each film.

It therefore seems feasible that in the not-too-distant future greatly improved Monte Carlo methods could be available which would lead to a better understanding of the basic processes in electron probe analysis and also serve as a working basis for more empirical but simpler methods to be applied in everyday use.

\section{Conclusions}

One of the most important problems outstanding in quantitative electron probe analysis is the determination of the statistically varying energy losses in metals. In the meantime, the advantages of a continuous energy loss model are considerable. The Bethe expression possibly in slightly modified form appears to be the most suitable for the purpose but serious attention must be given to the determination by theory and experiment of the excitation energy J. The most promising way of achieving a soundly based theory of the microprobe is probably by a Monte Carlo method with improved scattering cross sections and a modified stopping power relation as described above. In the meantime, less fundamental methods may be expected to profit from recent improvement in data and give quick and reliable results, provided that new ground is not being broken. In these empirical methods it is desirable, but not essential, that the basic theory and experimental data be the best available. The following preferred data are put forward as a basis for discussion.

a. Experimental data on the distribution of backscattered electrons by Bishop (1966) [2].

b. Backscattered fraction $\eta$ of electrons according to experiments by Bishop (this is supported by experiments by Mulvey and Page put not by Philibert and Weinryb). 
The combination of $a$ and $b$ leads to $R$ values that agree well with those determined experimentally by Castaing and Derian but not with those proposed by Poole and Thomas.

c. $f(\chi)$ values greater than 0.1 or 0.2 as determined by the Philibert/Duncumb-Shields model. For the smaller values than this $\mathrm{f}(\chi)$ values to be determined by models in which the ionization cross-section $Q_{k}$ is evaluated as a function of electron energy. Those determined by the Monte Carlo method of Bishop would be appropriate here, especially for light element analysis (cf. Duncumb and Melford 1965).

d. Scattering data that have proved useful in electron microscopy and electron diffraction should be used with caution in Monte Carlo calculations.

e. Nearly all the models suggested up to now have their place but it is important to define the limits of applicability.

f. Remarkable agreement between corrected and chemically determined composition is not necessarily proof that a model is soundly based.

g. As auxiliary data such as mass absorption coefficients are refined, the need for a sound theoretical model will become more rather then less urgent.

\section{References}

[1] Philibert, J. and Weinryb, E., Proc. Third International Symposium, X-Ray Optics and Microanalysis, Stanford, 1962, New York, Academic Press (1963) p. 451; see also, Weinryb, E., Thesis, University of Paris (1965).

[2] Bishop, H., Thesis, University of Cambridge (1966).

[3] Kulenkampff, H. and Spyra, W., Zeitschrift für Physik 137, 416 (1954); see also, Kulenkampff, H. and Ruttiger, K., Zeitschrift für Physik, 137, 426 (1954).

[4] Edwards, S. F., Bulletin of the Institute of Physics, London, April (1967).

[5] Castaing, R., Thesis, University of Paris (1950).

[6] Castaing, R. and Descamps, J., The Physical Basis of Electron Probe Microanalysis, J. Phys. Rad. 16, 304 (1955).

[7] Philibert, J., Proc. Third International Symposium, X-Ray Optics and Microanalysis, Stanford, 1962, New York, Academic Press (1963) p. 379

[8] Duncumb, P. and Shields, P., in "The Electron Microprobe", Effect of Critical Excitation Potential on the Absorption Correction, New york, John Wiley and Sons (1964) p. 284.

[9] Poole, D. M. and Thomas, P. M., in "The Electron Microprobe", Correction of Atomic Number Effects in Microprobe Analysis, New York, John Wiley and Sons (1964) p. 269.

[10] Derian, J. C., Détermination expérimentale du facteur de rétrodiffusion en microanalyse par émission X, Centre D'Etudes Nucleaires de Saclay, CEA Rept. R3052 (1966). 
[11] Halliday, J. S., Meeting on Quantitative Microprobe Analysis at the Institute of Physics (abstract only) (1962).

12] Wentzel, G., Zeitschr. Phys. 40, 590 (1927).

13] Lenz, F., Zeitschr. Naturforsch. 99, 185 (1954).

[14] Bethe, H., Annalen der Phys. 5, 325 (1930).

[15] Bethe, H., Handbuch der Physik 24, Berlin, Springer (1933) p. 273

[16] Duncumb, P. and da Casa, K., Institute of Physics Meeting, Recent Advances in Electron Probe Microanalysis, London, 1967 (abstract only).

[17] Duncumb, P. and Melford, D. A., Quantitative Application of Ultra-Soft X-Ray Microanalysis in Metallurgical Problems, International Congress, X-Ray Optics and X-Ray Microanalysis, Paris, September 1965. 



\title{
PROGRESS IN THE CORRECTION FOR THE ATOMIC NUMBER EFFECT
}

\author{
D. M. POOLE
}

Metallurgy Division, Atomic Energy Research Establishment, Harwell, Didcot, Berkshire, England

\section{Abstract}

The atomic number effect in electron-probe microanalysis results in a non-linear relation between generated $x$-ray intensity and composition; the paper describes those procedures for correcting microanalysis data which take into account this effect and compares their effectiveness by applying them to experimental data from various laboratories. Four procedures are found to be the best; namely those of Thomas (1963) [1] and Duncumb (1967) [25] for general use, and those of Belk (1964) [3] and of Ziebold (1964) [4] for use when absorption effects are not large.

\section{Introduction}

Mean atomic-number differences between alloy and standard in electron-probe microanalysis give rise to two factors which must be taken into account when converting intensity ratios into concentrations. These effects are (i) a non-linearity in the relation between primary generated $\mathrm{x}$-ray intensity and concentration, and (ii) a difference in distribution of the generated $\mathrm{x}$-rays between alloy and standard which gives rise to a difference in the absorption effect even if the absorption coefficients in the two materials are identical. This paper is concerned with the attempts which have been made over the years to deal with the first effect-i.e., the one which is by usage entitled the "Atomic Number Effect". The evolution of usable correction procedures will be traced, their principles outlined, and their relative merits assessed as far as possible by their application to experimental data obtained in various laboratories. These data consist of two batches, the first of 150 analyses of alloys of known composition and the second of an additional 79 analyses; histograms have been prepared of errors resulting from the use of various correction procedures on either the 150 cases, or, where further evidence was required, on the full 229 cases. (See Appendix 
for notes on the method of applying the corrections and on the preparation of the histograms.)

This discussion is restricted to work of a theoretical or practical nature which has led to the statement of a procedure which the practicing microanalyst can apply to his own data; it does not, therefore, make any attempt to review related work not leading to a working correction method.

\section{Discussions}

\section{A. THE ATOMIC NUMBER EFFECT}

For simplicity in the discussions which follow, let us consider the analysis of element $\mathrm{A}$ in a binary system $\mathrm{AB}$, the analysis being with reference to a pure standard of $A$; the data being obtained as the ratio, IA/I(A), (or K), of the intensity of the characteristic $x$-ray emission from the alloy to that from the pure standard, A. More complex situations involving multi-component systems, compound standards, etc., which arise in practice, can generally be dealt with by a simple extension of the binary method and are not discussed.

The physical situation can be visualized by reference to Figure 1 where the behavior of electrons incident on the alloy and standard is shown schematically; the alloy is supposed to be of a low mean atomic number $(\bar{Z})$ and the standard of a high $\mathrm{Z}$. A smaller fraction of electrons are backscattered from the alloy leading to a relative enhancement of the x-ray intensity from the alloy; on the other hand, the electrons lose energy more rapidly in the alloy, have less opportunity to produce ionizations, and thus generate a relatively reduced $x$-ray intensity. The balance of these two factors is such, that, in general, a low $\bar{Z}$ alloy referred to a high $\mathrm{Z}$ standard will have a generated intensity ratio less than the mass concentration of the analyzed element-i.e., the deceleration terms just predominate.

The generated $\mathrm{x}$-rays will be distributed in depth $(\rho z)$ somewhat as shown by the function $\phi(\rho z)$ in Figure 1; as noted above, the integrated intensity under the alloy curve is not directly proportional to concentration - - this being the atomic number effect as defined previously. The problem is to derive some means by which this overall enhancement or reduction effect can be estimated to enable the intensity ratios to be converted to concentration.

Furthermore, the distributions in alloy and standard are not of the same shape, thus giving rise to the atomic number contribution tc the absorption correction through the change in absorption patr lengths. 
respect there must be overlap between this paper and those dealing with the absorption itself.

Possible characteristic fluorescence effects, which can be quite important, have been disregarded in the discussion and the analyses used to test the methods are of systems where any such effects will be non-existent or negligible.

\section{B. PROPOSED METHODS OF CORRECTION}

The inter-relation between the many methods which have been developed over the last 12 years can conveniently be described by reference to the evolutionary plot given in Figure 2.

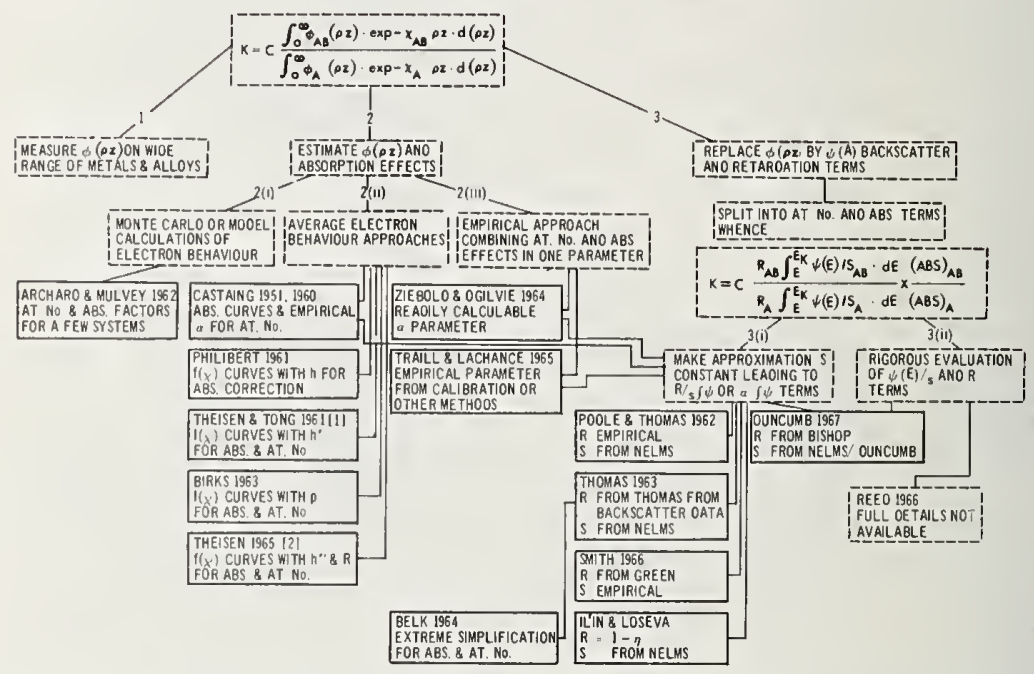

Figure 2. Evolutionary plot of methods for correction of the atomic number effect in electron probe microanalysis.

The overall objective of a correction procedure is to estimate the factor relating the intensity ratio to concentration, $\mathrm{C}^{A},{ }^{1}$ in the expression

$$
\mathrm{K}^{\mathrm{A}}=\mathrm{C}^{\mathrm{A}} \frac{\int_{0}^{\infty} \phi_{A B}(\rho \mathrm{z}) \cdot \exp -\chi_{A B} \rho \mathrm{zd}(\rho \mathrm{z})}{\int_{0}^{\infty} \phi_{A}(\rho \mathrm{z}) \cdot \exp -\chi_{A} \rho \mathrm{zd}(\rho \mathrm{z})}
$$

${ }^{1}$ Superscripts refer to the element or radiation concerned (normally A and omittec for clarity). Subscripts refer to the pure standard, A, or alloy, AB. 
This expression is exact and includes the two main corrections since each $\mathrm{d}(\rho \mathrm{z})$ element of generation (through $\phi)$ is linked to the appropriate absorption path (through $\chi$ ).

Three main routes can be proposed:

Firstly, (Route 1) measurements can be made by tracer experiments, of the type employed by Castaing (1960) [5], of the function $\phi(\rho \mathrm{z})$ for alloys and standards of interest so that equation (1) could be evaluated. The amount of effort involved would be impossibly large for general purposes and no such work has been attempted. However, it might well be worth while carrying out an examination of selected alloy systems as a direct check on some of the estimation methods proposed.

Secondly, (Route 2) there is the estimation, by various means, of the function $\phi(\rho z)$ and the associated absorption effect; this route has been extensively used and can be regarded as having three subroutes -2(i), 2(ii), and 2(iii) - covering calculations using models of individual electron behavior, descriptions of an average electron behavior by means of scattering parameters, etc., and empirical approaches which combine the whole correction into a single parameter.

Thirdly, (Route 3 ) there is the approach whereby the x-ray intensity and distribution is considered as dependent on electron backscatter and retardation terms and on the ionization cross section $\psi(\mathrm{E})$; the absorption is evaluated separately. Using these concepts, there is then a choice as to the degree of precision with which the factors are evaluated-roughly speaking, two branches have developed: 3(i) in which approximate solutions have been sought and 3(ii) in which a more rigorous approach has been adopted.

We may now consider these routes in turn and the procedures which have been allocated to them.

Route 2(i). Estimation of $\phi(\rho \mathrm{z})$ from a consideration of individual electron behavior can be carried out in various ways. For example, a large number of electrons can be followed through a large number of interactions with the target material by a Monte Carlo type calculation, or simplifications can be introduced to make the calculation more manageable. An example of the latter approach is that of Archard and Mulvey (1962) [6] in which a relatively simple model of the behavior of a selection of individual electrons was used; the model is one which has already been shown (Archard 
(1961) [7]) to represent the backscatter process adequately. A simple extension enabled $\mathrm{x}$-ray yield and absorption effects of equation (1) to be calculated for selected metal and alloy targets under various excitation conditions from which curves were obtained of the variation with composition of both the atomic number and absorption effects in a few alloy systems.

Strictly, the correction factor curves can only be used for those systems to which they refer and the method cannot be generally used as it stands unless the user is prepared to perform the calcu. lations for his own particular cases. However, an attempt has beer made to approximate and interpolate between the published curves in order to perform some sort of correction to all 150 cases: (the error distribution diagram of the uncorrected data is shown in Fig ure 3 ) the resulting histogram is shown in Figure 4 but should no have much reliance placed upon it in view of the interpolation: employed to produce it. Figure 5, however, shows those case where little or no departure from the published curves was involver and considerable residual errors are still obvious here; in the absence of further calculations of the corrections for actual experi mental situations, it is not possible to judge the method fairly. $I^{\dagger}$ is to be hoped that the authors will find some means of carrying through these additional calculations.

Route 2(ii). This route contains those methods which havi evolved from consideration of the form of the $\phi(\rho z)$ distribution function; however, the curious point emerges that all the workabl procedures involve the introduction of factors into the absorptiol terms in order to correct for the atomic number effect, even thougl the latter is really one of generation, i.e., the correction for $\phi(\rho z$ shape is being modified to attempt to correct for area differences.

The pioneer work of Castaing (1951) [8] and Castaing an Descamps (1955) [9], measuring $\phi(\rho z)$ and laying the theoretice? basis for further work, must be mentioned here although it did nc lead directly to a usable procedure. In his thesis, Castaing (1951 [8] introduced an empirical $\alpha$ term describing the properties of eac element such that the generated intensity ratio, $\mathrm{K}^{\prime}$, was given by:

$$
\mathrm{K}^{\prime}=\left(\mathrm{C}^{A} \alpha_{A} / \mathrm{C}^{A} \alpha_{A}+\mathrm{C}^{B} \alpha_{B}\right)
$$

This expression involving the assumption, among others, that

$$
\left(\int_{o}^{\infty} \phi_{A}(\rho \mathrm{z}) \mathrm{d}(\rho \mathrm{z}) / \int_{0}^{\infty} \phi_{B}(\rho \mathrm{z}) \mathrm{d}(\rho \mathrm{z})\right)=\left(\alpha_{B} / \alpha_{A}\right)
$$


the various $\phi_{A}, \phi_{B}$ here describing radiation $\mathrm{A}$ in pure $\mathrm{A}$, or the same radiation in effectively pure $B$. In his later publication, Castaing (1960) [5] suggests that $\alpha$ is the product of two Z-dependent factors: $\lambda$, a coefficient describing the effect of backscatter, and a deceleration term taken at that time as Z/A following Webster (1928) [10]. Here, and in equation (2), we have the forerunner of the methods later discussed under Route 3(i) and Castaing's work might equally be mentioned under that heading.

Absorption terms, $\mathrm{F}(\chi)$ or $\mathrm{f}(\chi)$ according to assumptions made, ${ }^{2}$ can then be introduced such that the emitted intensity ratio, $\mathrm{K}$, is related to the generated intensity by:

$$
\mathrm{K}=\mathrm{K}^{\prime}\left(\mathrm{F}(\chi)_{A B} / \mathrm{F}(\chi)_{A}\right) \quad .
$$

Philibert (1961 on) [11] derived an expression for $F(\chi)$ from an electron distribution model which enabled the x-ray distribution function to be estimated in various target materials. His expression

$$
1 / \mathrm{F}(\chi)=(1+\chi / \sigma)[1+\mathrm{h}(1+\chi / \sigma)]
$$

lintroduced the parameter $h\left(=1.2 \mathrm{~A} / \mathrm{Z}^{2}\right)$ which enabled the function to be calculated for any pure metal target ( $\sigma$ is a Lenard coefficient dependent on incident voltage); by introducing an alloy $h$, calculated from the mean $\mathrm{A} / \mathrm{Z}^{2}$ value for the alloy, the absorption function for the alloy target could also be calculated. The parameter $\mathrm{h}$ takes into account the atomic number part of the absorption correction but does not, however, constitute a correction for the atomic number effect as such, although at the time this was not universally appreciated.

About the same time, Theisen (1961) [12] and Tong (1961) [13] produced expressions for $F(\chi)$, exactly similar to those of Philibert, which were claimed to perform the whole of the necessary forrection from intensity ratio to concentration when used in the quation

$$
\mathrm{K}=\mathrm{C}\left(\mathrm{F}(\chi)_{A B} / \mathrm{F}(\chi)_{A}\right)
$$

he parameter $\mathrm{h}$ (called $\xi$ ) in Theisen's expression was evaluated rom

$$
\mathrm{h}=3.5 \mathrm{~A} / \mathrm{Z}^{2}
$$

$F(\chi)$ and $f(\chi)$ are related by the the expression $f(\chi)=F(\chi) / F(0)$ where $F(0)$ refers to $\mathrm{F}(\chi)$ when $\chi=0$. 
The results quoted by Theisen in support of his expression were good but despite this the histogram for 150 cases (Fig. 6) shows quite large errors. Heavy-in-light element combinations constitute $87 \%$ of the 150 cases and the atomic number effect, if uncorrected, would bias the error distribution to the negative side; some evidence of this bias remains in this histogram suggesting that the effect has not been fully compensated for.

In his book, Birks (1963) [14] put forward a simplified method of absorption correction into which he introduced a factor $\mathrm{p}$, dependent on $\mathrm{Z}$, which was used to adjust the value of $\chi$ before reading $F(\chi)$ from the tables provided; the $F(\chi)$ values were then used in a simple expression identical to equation (6). This procedure was ". . . to make a correction for the variation of $\phi(\rho \mathrm{z})$ " and was claimed to perform the atomic number correction. Using this method on the 150 cases produces the histogram in Figure 7 from which the errors are seen to be large and biased heavily to negative values; the bias indicates that the atomic number effect is not being corrected for.

Two years later, Theisen (1965) [15] produced his book in which his 1961 correction formula had been considerably modified to:

$$
\mathrm{K}=\mathrm{C}\left(\mathrm{R}_{A B} / \mathrm{R}_{A}\right)\left(\mathrm{f}(\chi)_{A B} / \mathrm{f}\left(\chi_{A}\right)\right.
$$

where $\mathrm{R}$ is an effective current factor (after Thomas (1963) [1]) tc allow for backscatter loss and $f(\chi)$ is used (instead of $F(\chi)$ ) and is calculated from

$$
1 / \mathrm{f}(\chi)=\left(1+\chi / \sigma_{E}\right)\left[1+\left(\frac{\mathrm{h}}{1+\mathrm{h}}\right) \chi / \sigma_{E}\right]
$$

where $\sigma_{E}$ is a modified Lenard coefficient taking into accoun overvoltage:

$$
\sigma_{E}=\left(8.9 \times 10^{5} /\left(\mathrm{V}-\mathrm{V}_{c}\right)^{2}\right)
$$

and $\mathrm{h}$ now combined atomic number and voltage terms in th expression

$$
\mathrm{h}=1.72 \times 10^{-6} \mathrm{~V}^{2} \mathrm{~A} / \mathrm{Z}^{2}
$$

Application of the method to 40 of the available cases suggesf that the method is less satisfactory than the earlie version-disregarding sign, the mean value of the apparent error $i$ now $16 \%$ compared to $8 \%$ for the same cases treated by Theise 
12], and $4 \%$ by the Thomas method. This is hardly surprising as ve are introducing now only one of the two factors (backscatter ind retardation) which to a large extent counterbalance each other $\mathrm{n}$ theory and in practice. The result of this is that the reduction in ipparent concentration of heavy element analyses by the deceleraion effect is not corrected for and large negative errors appear in hese cases; similarly, analyses of the lighter elements of the binary combinations show large positive errors. This bias in the sign of he resulting errors can be seen from the following table:

Total no. Negative errors Positive errors

Heavy in light

Light in heavy
26

13
25

0
1

12

In view of the obvious inadequacies of this method, it has not een thought worthwhile to apply it to the full 150 cases and no istogram has therefore been prepared.

Route 2(iii). Ziebold and Ogilvie (1964) [16] proposed a method y which both the atomic number and the absorption effects were ombined into a single parameter, $\alpha$. This was employed in the elation

$$
(1-\mathrm{K}) / \mathrm{K}=a(1-\mathrm{C}) / \mathrm{C}
$$

he derivation of the expression by which $\alpha$ may be readily alculated has its origins in Route 3 -i.e., it takes into consideration ackscatter and retardation terms - but its method of combining the tomic number and absorption effects into a single parameter leads $p$ its discussion under the present head. The expression for $\alpha$ is:

$$
\alpha=0.95\left(\frac{\sigma+\chi_{B}}{\sigma+\chi_{A}}\right)\left(\frac{Z_{A}}{Z_{B}}\right)^{0.28} .
$$

The histogram of errors for the 150 cases is in Figure 8 and hows a generally satisfactory distribution in view of accepted hcertainties in some of the data; virtually no skewness remains hd the atomic number effect would seem to be well accounted for. The method was further applied to the additional 79 cases and the 9-case histogram is given in Figure 13.

A second method relying on a single parameter to combine both ajor corrections is that of Traill and Lachance $(1965)[17,18]$ who 
discuss the general area of spectrochemical analysis of multicomponent systems; they demonstrate that the expression

$$
\mathrm{K}=\mathrm{C}^{\mathrm{A}} /\left(1+\mathrm{C}^{\mathrm{B}} \alpha_{A B}\right)
$$

adequately represents observed intensity ratios in selected analytical examples when $\alpha_{A B}$ is chosen to suit the particular system investigated. The evaluation of $\alpha$ can either be by calibration or by a combination of absorption, atomic number and fluorescence corrections calculated by some such approach as those in Route 3; to this extent the method would not seem to have any great advantage except perhaps for work involving a calibration procedure. However, a special value may lie in the claim that multi-component systems can readily be dealt with by deriving a combined $\alpha$ parameter from the $\alpha$ 's for the constituent binary systems; this capability is also a feature of Ziebold and Ogilvie's method.

The general similarity to Ziebold and Ogilvie's approach is such that the development of a separate histogram for this method was not thought to be necessary.

Route 3(i). Methods which are considered as belonging to this route involve approximating to the initial intensity ratio by an expression involving atomic number and absorption terms separately, viz:

$$
\begin{aligned}
& \mathrm{K}=\mathrm{C} \frac{\mathrm{R}_{A B} \int_{\mathrm{E}}^{\mathrm{E}_{K} \psi_{A B}(\mathrm{E}) / \mathrm{S}_{A B}(\mathrm{E}) \mathrm{dE}}}{\mathrm{R}_{A} \int_{\mathrm{E}}^{\mathrm{E}_{K}} \psi_{A}(\mathrm{E}) / \mathrm{S}_{A}(\mathrm{E}) \mathrm{dE}} \times \frac{\text { (Absorption Factor) })_{A B}}{\text { (Absorption Factor) })_{A}} \\
& \text { Atomic number } \\
& \text { Absorption . (15 }
\end{aligned}
$$

Here $\mathrm{R}$ is the effective current factor, already introduced, whic takes account of the loss of ionization due to backscatter, $\psi(\mathrm{E})$ is th ionization cross section for the radiation concerned and $S(E)$ is th stopping power of the target for electrons of energy $E$ (define as $-(1 / \rho)(\mathrm{dE} / \mathrm{dx}))$. Approximations and empirical factors are the introduced to enable the atomic number part of expression 15 to $b$ evaluated.

In their first method, Poole and Thomas (1962) [19] followed th lead offered by Castaing's early work and by assuming that $S$ we constant (and so placeable outside the integral) and that

$$
\int \psi_{A B}(\mathrm{E}) / \int \psi_{A}(\mathrm{E})=1
$$


they reduced equation (15) to the form

$$
\mathrm{K}=\mathrm{C} \quad \frac{\mathrm{R}_{A B}}{\mathrm{~S}_{A B}} \frac{\mathrm{S}_{A}}{\mathrm{R}_{A}} \frac{\text { (Absorption function) })_{A B}}{\text { (Absorption function) }}
$$

or $\quad \mathrm{K}=\mathrm{C} \quad \frac{a_{A}}{\mathrm{C}^{\mathrm{A}} a_{A}+\mathrm{C}^{\mathrm{B}} a_{B}} \frac{\mathrm{F}(\chi)_{A B}}{\mathrm{~F}\left(\chi_{A}\right.}$

which is identical to the combination of Castaing's equations 2 and 4 and which assumes that

$$
\left(\mathrm{S}_{A B} / \mathrm{R}_{A B}\right)=\alpha_{A B}=\mathrm{C}^{A} \alpha_{A}+\mathrm{C}^{B} \alpha_{B}
$$

with $\quad \mathrm{S}_{A} / \mathrm{R}_{A}=\alpha_{A}$ and $\mathrm{S}_{B} / \mathrm{R}_{B}=\alpha_{B}$

$S$ was supposed equal to $S$ from Nelms' tabulation [20] taken at $30 \mathrm{kV}$, and $\mathrm{R}$ was taken from an empirical curve of the variation with $\mathrm{Z}$ which had been estimated from the results of a few analyses. At this stage the proposed correction for atomic number effect takes no account of excitation conditions.

This method was quite promising but a histogram is not given here as it was soon succeeded by the revised method of Thomas (1963) [1] which takes account of the excitation conditions. In this version, equation (17) was still used but $R$ was taken from curves of $\mathrm{R}$ versus $\mathrm{Z}$ and overvoltage $\left(\mathrm{E}_{o} / \mathrm{E}_{c}\right)$ which had been estimated py a rough approximation from published backscatter data; $S$ was still obtained from Nelms' tables but was taken at $E=\left(E_{o}+E_{c}\right) / 2$. The procedure also involved a special way of attempting to allow jor the atomic number part of the absorption correction-namely he use of the same value of $h\left(=h_{A}\right)$ in Philibert's expression for $(\chi)$ for both alloy and standard, coupled with a modification of $A B$ to the form

$$
\chi_{A B}^{\prime}=\frac{\chi_{A B}}{C^{A}+C^{B}\left(\alpha_{B} / \alpha_{A}\right)}
$$

his procedure has not been universally accepted as reasonable; evertheless, the method works well as the histogram of 150 results $n$ Figure 9 shows.

Extension to the full 229 cases produces the histogram of igure 14 . 


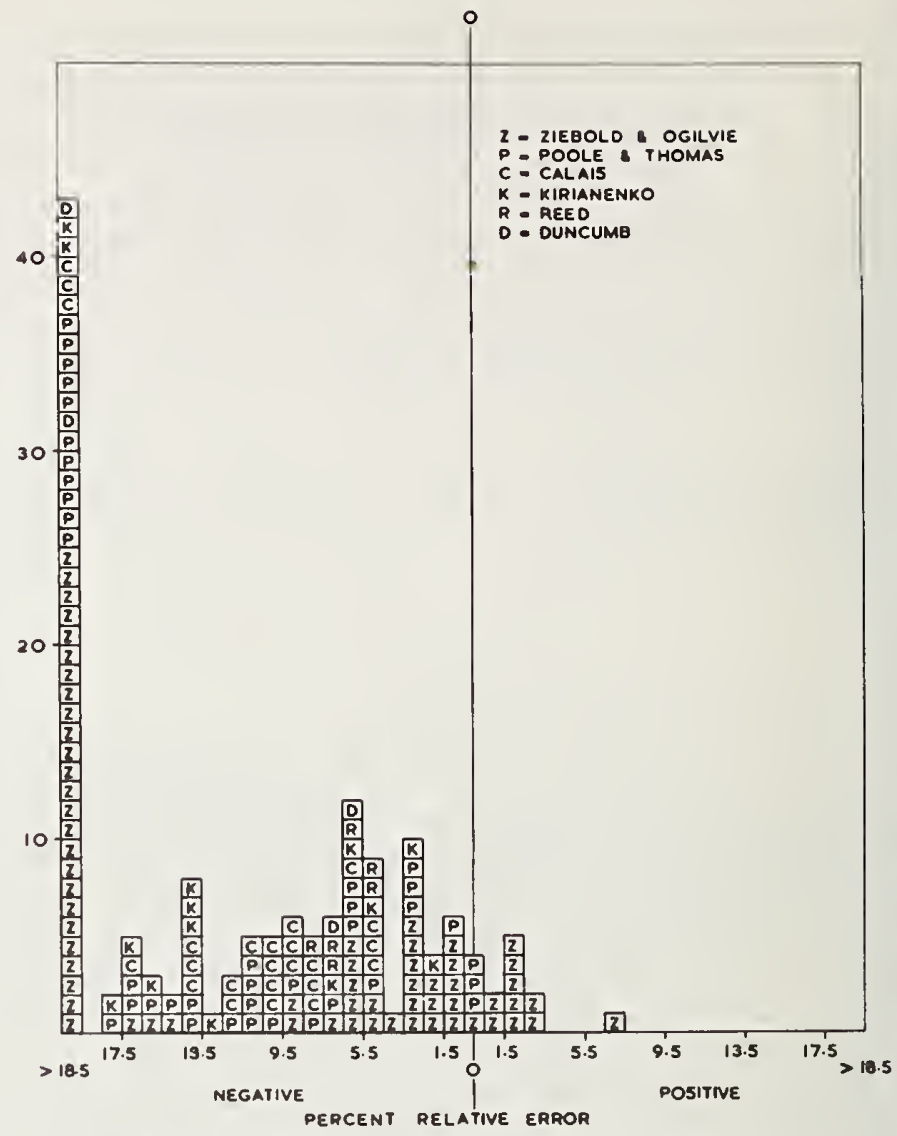

Figure 3. Error Histogram - 150 cases, uncorrected data.

Working from the voltage-independent atomic number correction of the type proposed by Poole and Thomas, and from Philibert' absorption correction procedure, Belk (1964) [3] produced a gros simplification enabling the complete correction to be reduced to

$$
\mathrm{C}=\mathrm{K} \frac{\left[1+\frac{\mathrm{Z}_{A}-\overline{\mathrm{Z}}_{A B}}{100}\right]}{\text { Atomic Number }} \frac{\left[1+\frac{\mu_{A B}-\mu_{A}}{\mathrm{P}+\mu_{A}}\right]}{\text { Absorption }}
$$

where

$$
\mathrm{P}=\sigma \sin \theta[(1+\mathrm{h}) /(1+2 \mathrm{~h})]
$$

and if the atomic number $\mathrm{Z}$ of any component exceeds 40 , it shoulc 


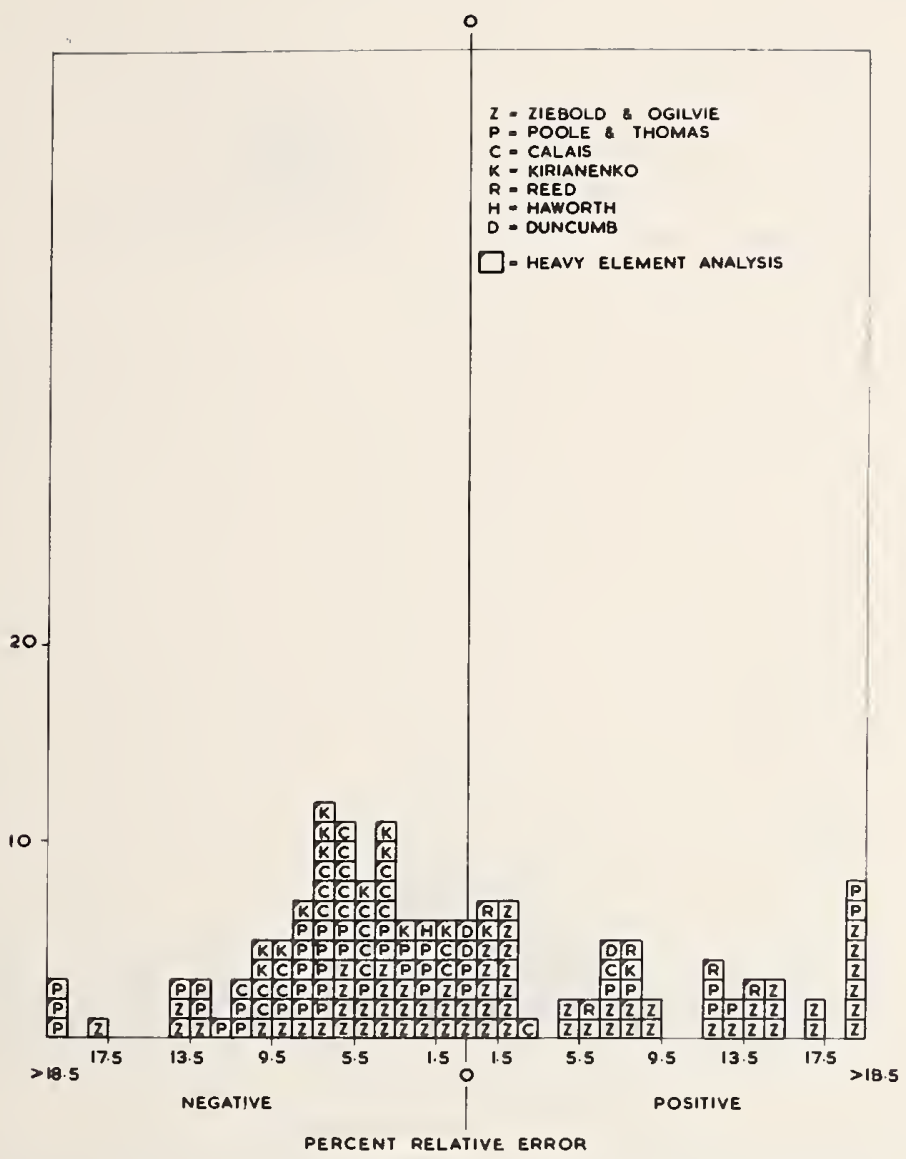

Figure 4. Error Histogram-150 cases, Archard and Mulvey method.

be represented by a $Z^{\prime}=40+0.3(Z-40)$. The method will not work well when the absorption correction is large and Belk has set limits outside which errors greater than $2 \%$ can be expected. Of the 150 cases, 128 are such as to be inside these limits and Belk has obtained for these cases the histogram which is shown in Figure 10. Points outside Belk's $2 \%$ limits have been added in distinguishing shading. Despite its extreme simplicity, the method clearly works very well and compares favorably with the more complicated methods.

Application of the method to all 229 cases gives the histogram of Figure 15; again, points corresponding to cases which are outside Belk's limits are marked.

Il'in and Loseva (1966) [21] have evolved an exactly similar expression to equation (17). In this instance, however, $\mathrm{R}$ is set equal to $(1-\eta)$ where $\eta$ is the backscatter coefficient and $S$ is 


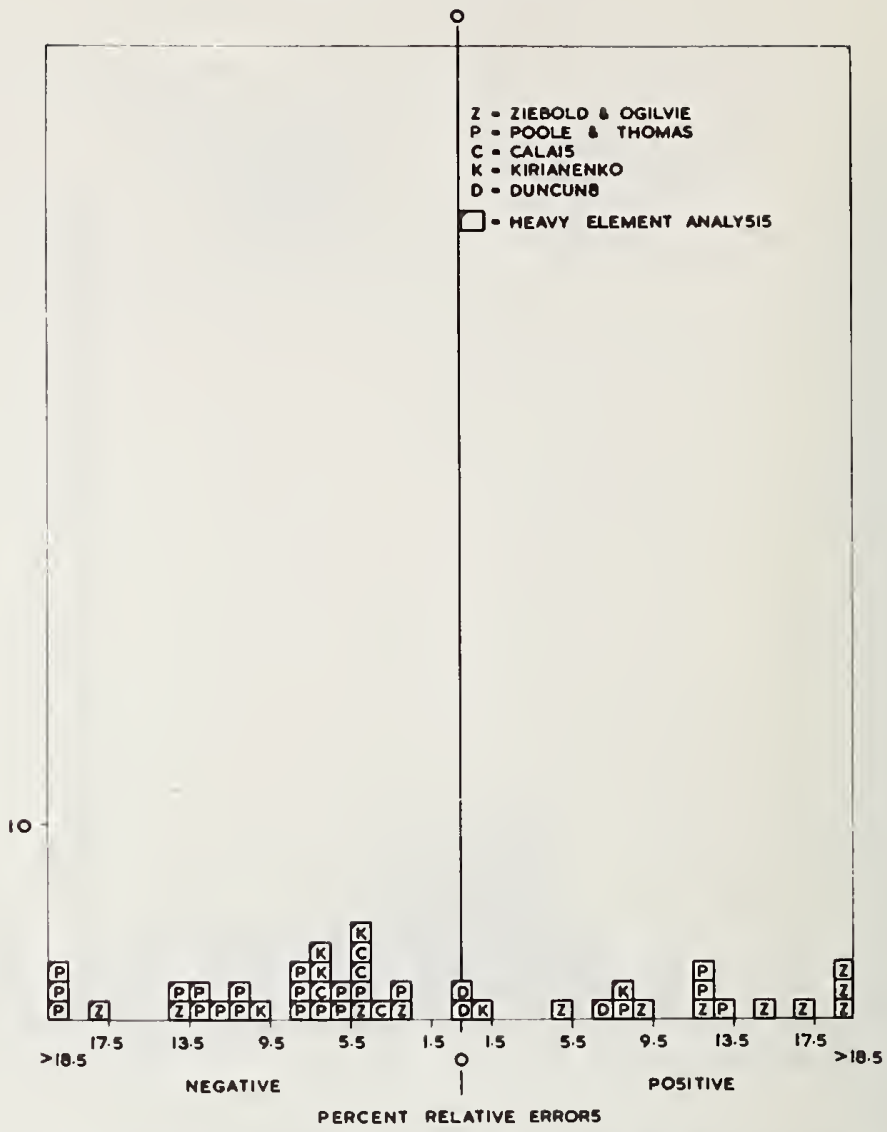

Figure 5. Error Histogram-150 cases, Archard and Mulvey method, selected cases.

again taken from Nelms' tables. Mean values of $\mathrm{S}$ and $\mathrm{R}$ are calculated separately for the alloy by weight fraction. The early work of Poole and Thomas has already shown that experimental observations indicate a value of $\xi$ in the equation $\mathrm{R}=(1-\xi \eta)$ which is far from unity and hence, it seems certain that Il'in's method will not give results of a good accuracy. On theoretical grounds, too, it is clear that the backscatter ionization loss $(1-\mathrm{R})$ will not directly equal $\eta$, as Il'in assumes, since many of the backscattered electrons will already have contributed to the The absorption factors are computed from a simple expression involving only the absorption coefficients of alloy and standard and the concept of a mean depth of emission-this depth is either estimated from $\mathrm{E}$ and $\mathrm{E}_{o}$ or obtained by calibration. The method has not yet been applied to the test cases. 


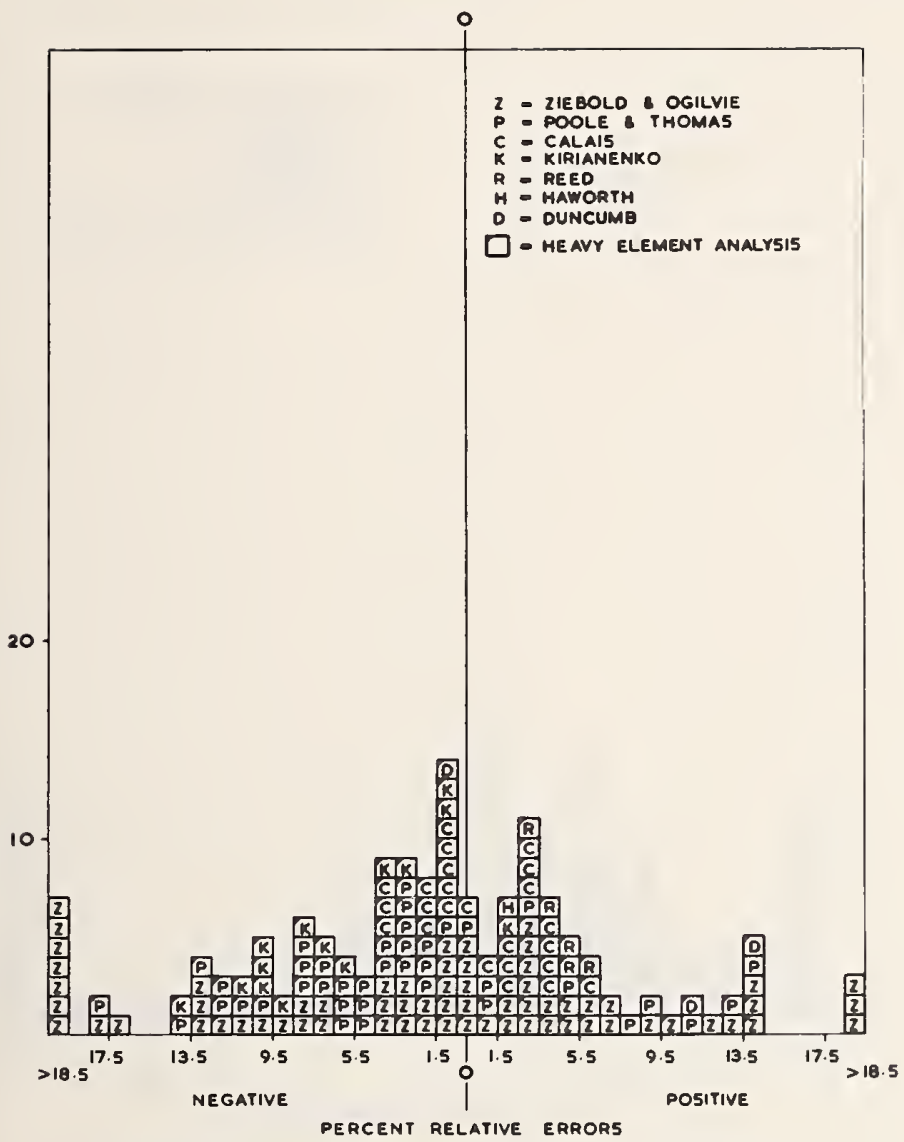

Figure 6. Error Histogram - 150 cases, Theisen method.

Smith (1965) [22] has also evolved a method for evaluating the terms S/R in the correction equation (17); he has utilized the more rigorously determined $R$ curves of Green (1963) [23] and an empirical expression for $\mathrm{S}$ (based on one first proposed by Long) namely

$$
\left.\mathrm{S}=\left[4.7+0.9 \ln \left(\mathrm{E}_{o}-\mathrm{E}_{c}\right)-\ln \langle\mathrm{Z}\rangle\right]<\frac{\mathrm{Z}}{\mathrm{A}}\right\rangle
$$

where $\langle>$ denotes a mean value appropriate to alloy or standard. These terms are put forward for use with a set of mass absorption coefficients which have been specially derived to fit a large number of microanalysis results from mineralogical samples. The tabulation of coefficients are not extensive enough to enable Smith's method to be rigidly applied to all our test cases; however, the departure of the 


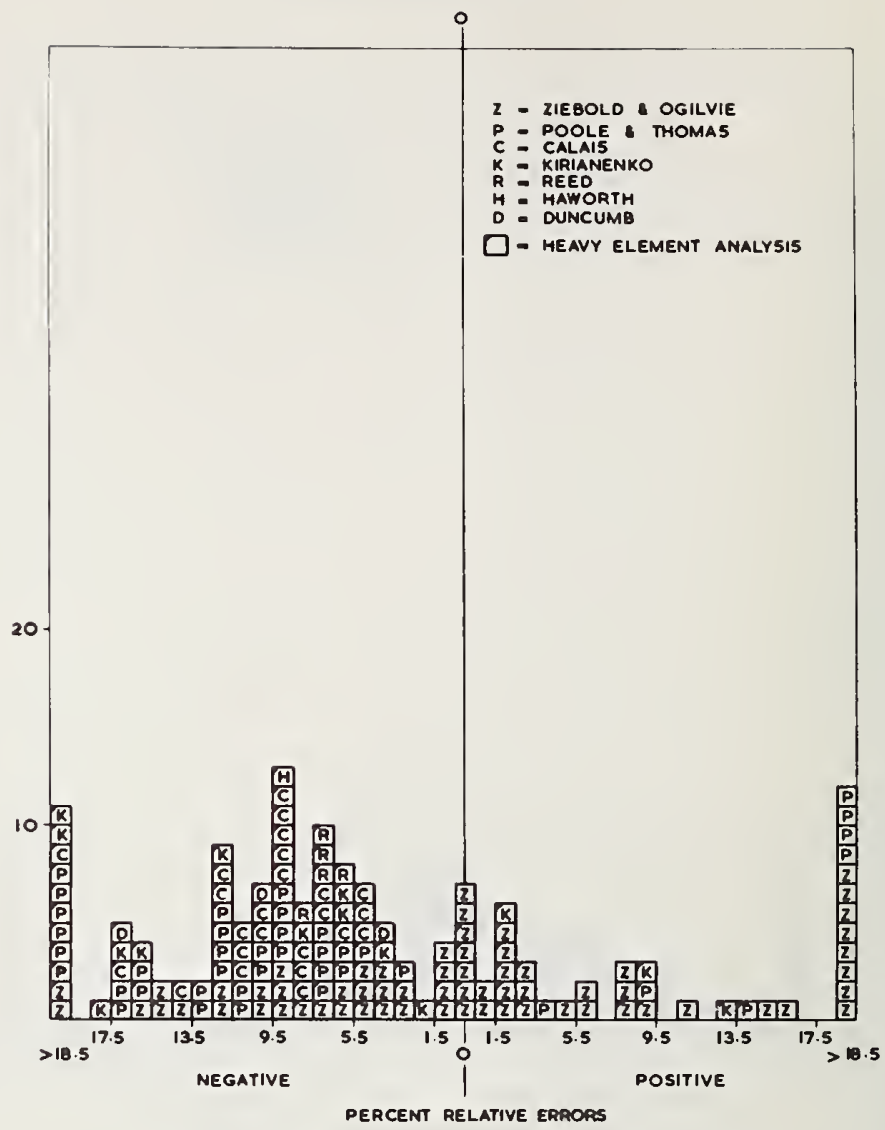

Figure 7. Error Histogram-150 cases, Birks method.

values from those given by Heinrich [24] are in general not significant except for the soft radiations and Heinrich's values have been used in evaluating Smith's method as shown in Figure 11 for the 150 cases.

Extension of the method to the 229 cases is shown in Figure 16.

Route 3(ii). A more rigorous approach has been adopted by Duncumb [2] who has carried through accurate evaluations of the expression $\int \psi(\mathrm{E}) / \mathrm{S}(\mathrm{E}) \mathrm{dE}$ and of the backscatter loss. By this means, he has been able to estimate the errors involved ir approximations and simplifications which have been proposed to enable the correction to be simply carried out; for example, he ha: shown that the assumption of S constant, which is implicit in all the Route 3(i) methods, is one which in general introduces only a smal 


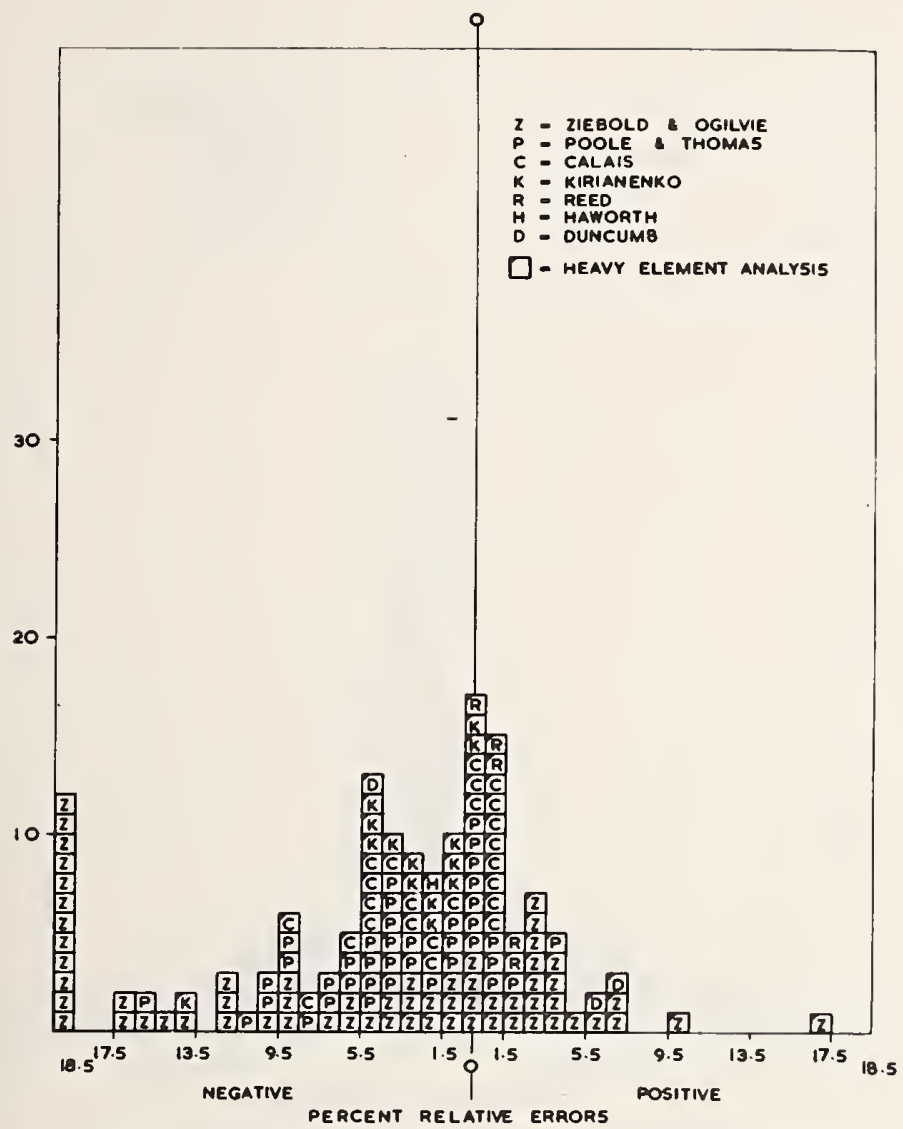

Figure 8. Error Histogram - 150 cases, Ziebold method.

error. As well as looking into the finer details, this work has also produced a readily used method-Duncumb (1967) [25]. In this, the familiar $\mathrm{S}$ and $\mathrm{R}$ terms are employed in an equation identical to (17); $\mathrm{R}$ is taken from the curves recently produced by Bishop (1966) [26] and $S$ is evaluated from the expression

$$
\mathrm{S}=\text { const. }(\overline{\mathrm{Z} / \mathrm{A}})(1 / \overline{\mathrm{E}}) \ln (1.166 \overline{\mathrm{E}} / \mathrm{J})
$$

where $\mathbf{J}$ is the mean ionization potential for the target material and is evaluated from an empirical relation:

$$
\mathrm{J} / \mathrm{Z}=15.05\left[1-\mathrm{e}^{-0.072 \mathrm{Z}}\right]+\left(42 / \mathrm{Z}^{Z / 10}\right)-(\mathrm{Z} / 400)
$$

Equation (26) was evolved to give values of $\mathbf{J}$ which resulted in the best possible fit between corrected analytical results and known concentration for selected alloys - i.e., microanalytical results have 


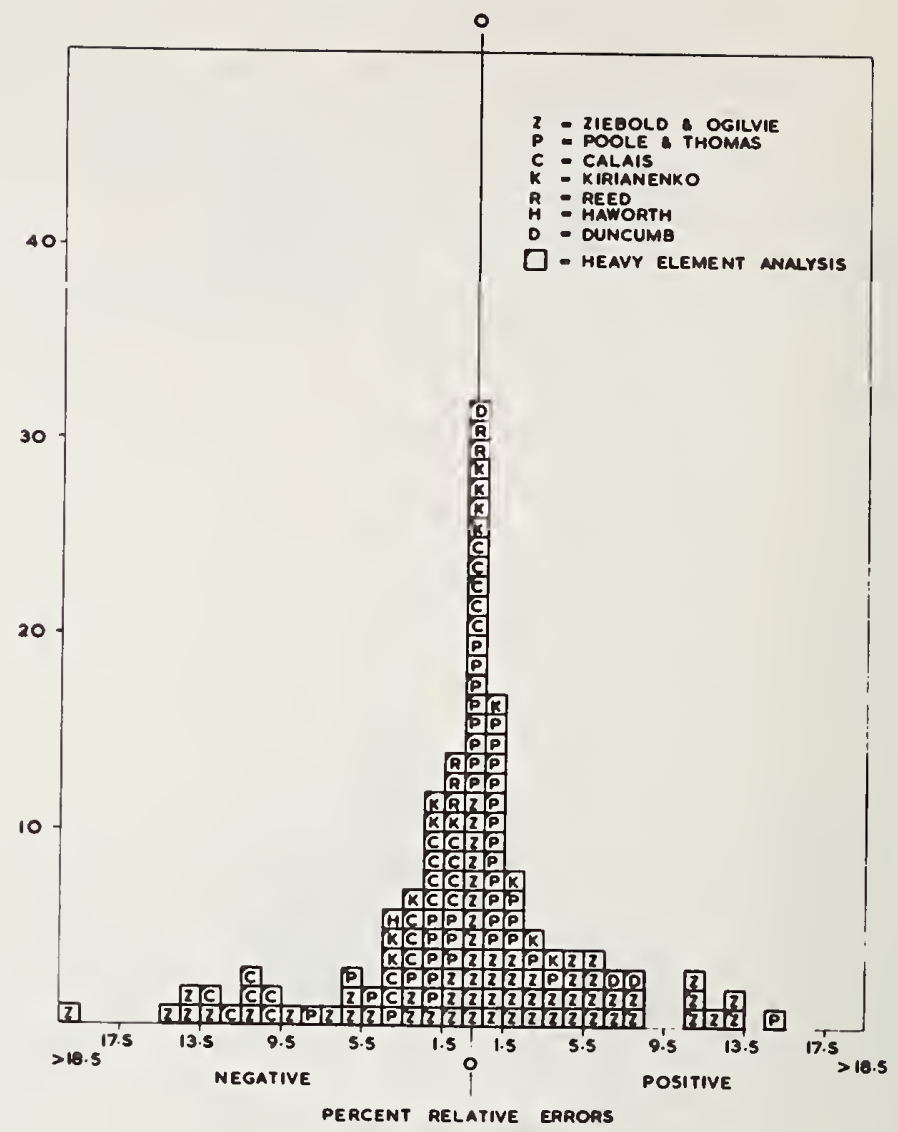

Figure 9. Error Histogram - 150 cases, Thomas method.

been used to determine the apparent variation of the physical parameter, J. The absorption correction is carried out using Philibert's $\mathrm{f}(\chi)$ formula as modified by Duncumb to take account of overvoltage.

Application of the method to the 150 cases produces the histogram in Figure 12; in Figure 17, the extended histogram for the 229 cases is shown. It is clear that the method is among the more successful.

\section{Conclusions}

The general progress achieved by the various methods developec from 1961 onwards can be assessed by reference to the histograms for the 150 cases in Figures 4-12; the differences in the success 0 the various methods are emphasized by the tabulation (Table 1) o 


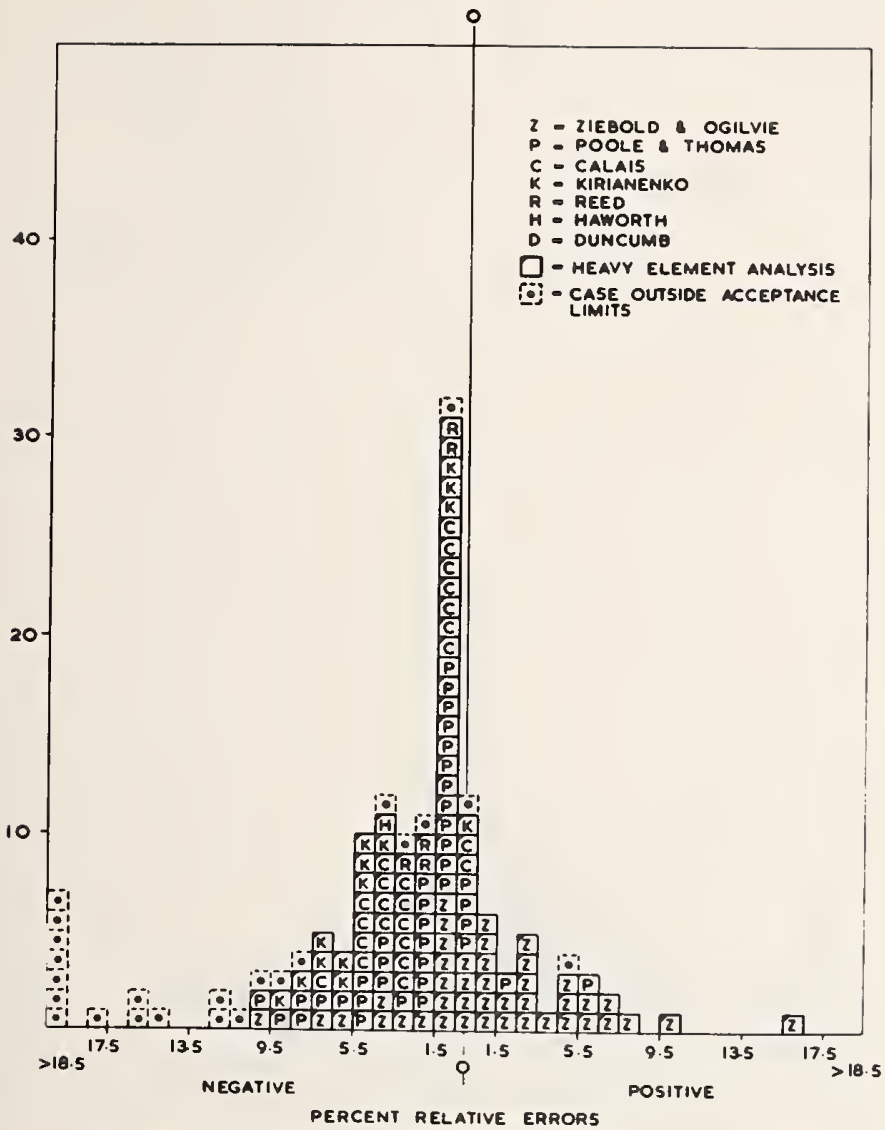

Figure 10. Error Histogram-150 cases, Belk method.

errors up to $10 \%$ are common, and only $27 \%$ of cases fall with $\pm 2 \frac{1}{2} \%$.

The first comprehensive method of Theisen (1961) [12] can be seen to have achieved some measure of success in that the residual errors do not show any of the bias which would arise from failure to correct for a particular factor; however, the histogram is broad, the percentage of cases for each method which fall with the limits $\pm 2 \frac{1}{1} \%$.

The basically more complex method of Archard and Mulvey [6] introduced in 1962 does not show any better results on the present issessment with only $22 \%$ of cases falling within $\pm 21 / 2 \%$; in the absence of further evidence to the contrary, this cannot be regarded as a successful approach as far as general application is concerned.

In 1963, Birks [14] published his method which reduced the correction to very simple steps suitable for hand calculation. The 


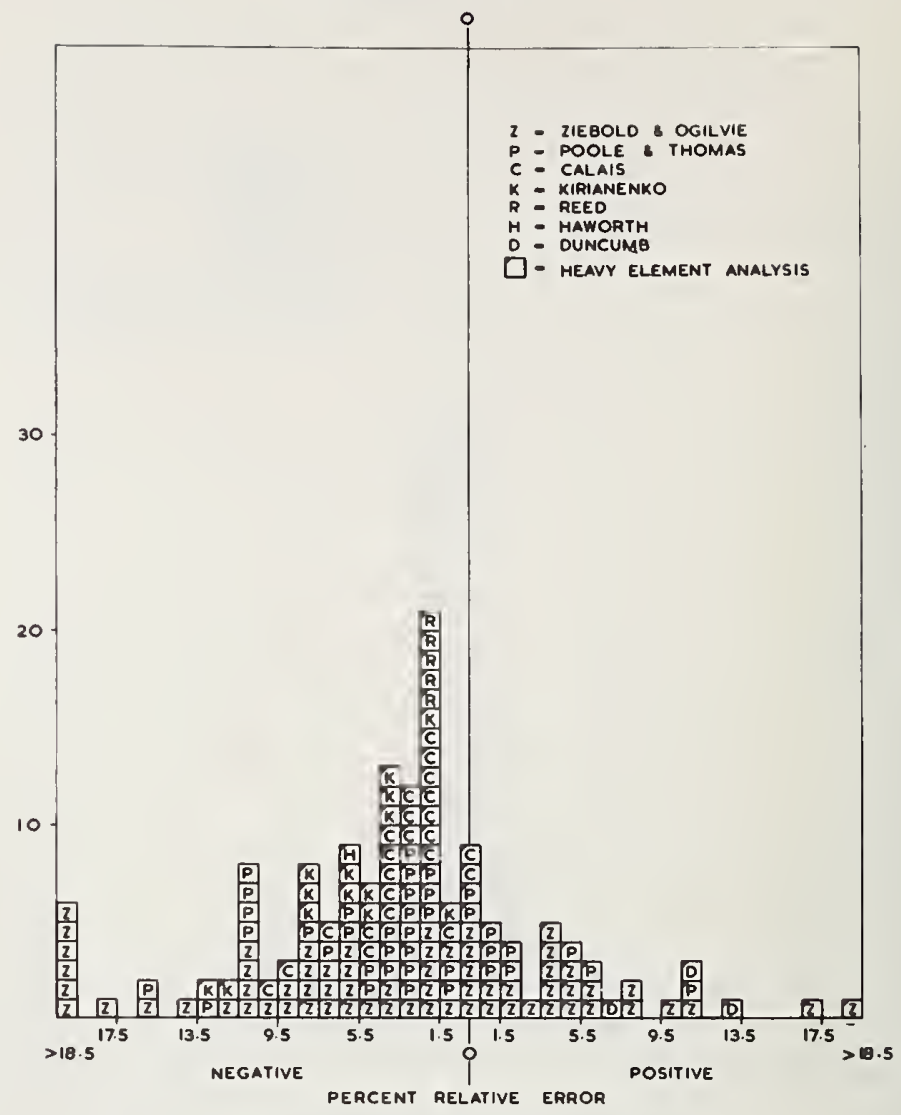

Figure 11. Error Histogram - 150 cases, Smith method.

histogram shows that the atomic number effect was not successfully dealt with and the advantage of simplicity is outweighed by the large errors resulting; only $13 \%$ of analyses are corrected to within the $2 \frac{1}{2} \%$ limits.

The method of Thomas [1] was also published in 1963 and shows a big step forward in effectiveness; the atomic number factors are well corrected for and $55 \%$ of the cases fall within $\pm 21 / 2 \%$. The procedure can be carried through by hand but can be readily computerized if required.

The extremely simple method introduced by Belk [3] in 1964 car be seen from the histogram to be remarkably successful; some of the cases outside the prescribed absorption correction limits are reasonably accurate and even including all such cases in the coun $42 \%$ of the results fall within $\pm 2 \frac{1}{2} \%$. Where absorption effects cal 


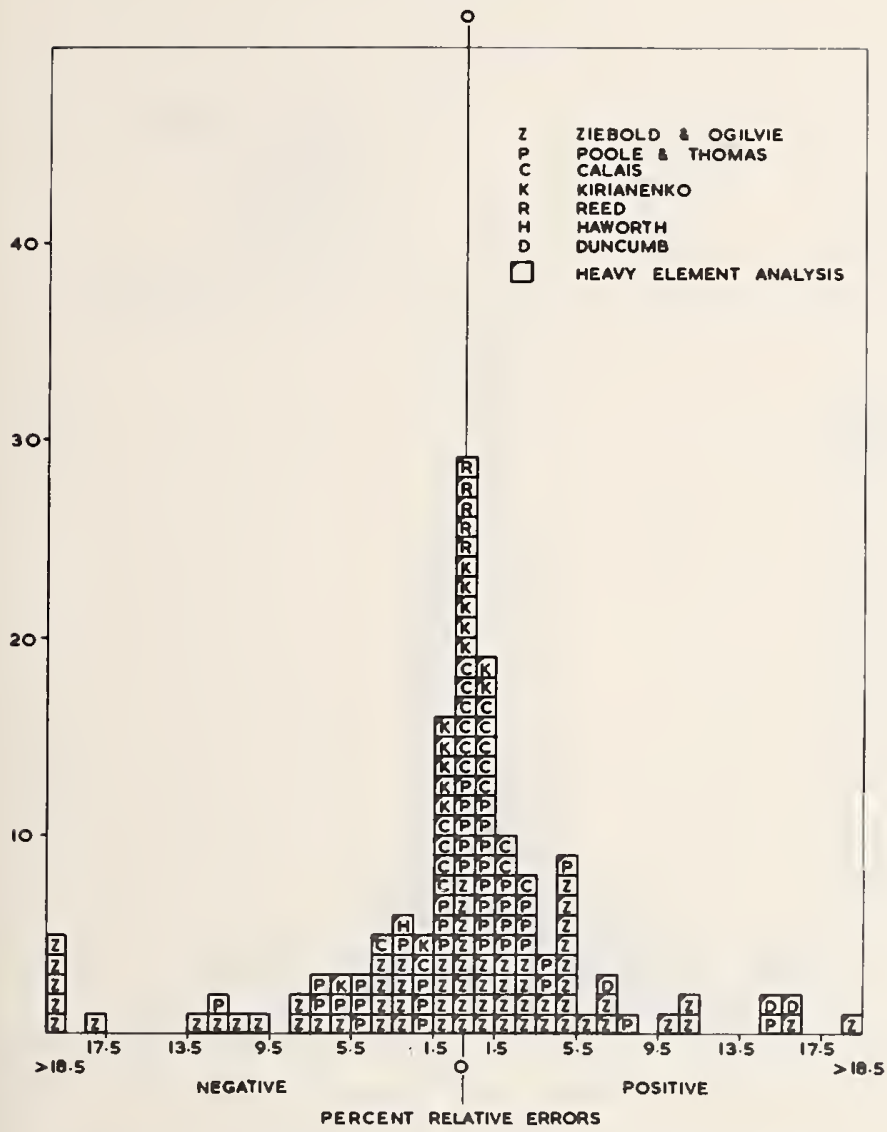

Figure 12. Error Histogram-150 cases, Duncumb method.

be kept small, by suitable choice of voltage for example, and where a quick, easy correction is required then this is obviously a suitable method.

Ziebold and Ogilvie's method [4] introduced in 1964 also provides a simply used method which seems, however, to be slightly less effective than Belk's with $37 \%$ of cases better than $2 \frac{1}{2} \%$.

The methods of Traill and Lachance [17,18] and of Theisen [15], introduced in 1965, did not improve on the existing array of procedures, nor did that of Il'in and Loseva [21] introduced in 1966.

Duncumb [25] had been working on his method for some time before its nominal date of 1966 and it can be seen from the histogram that it is very successful-some $53 \%$ of cases fall within $\pm 2 \quad 1 / 2 \%$. 


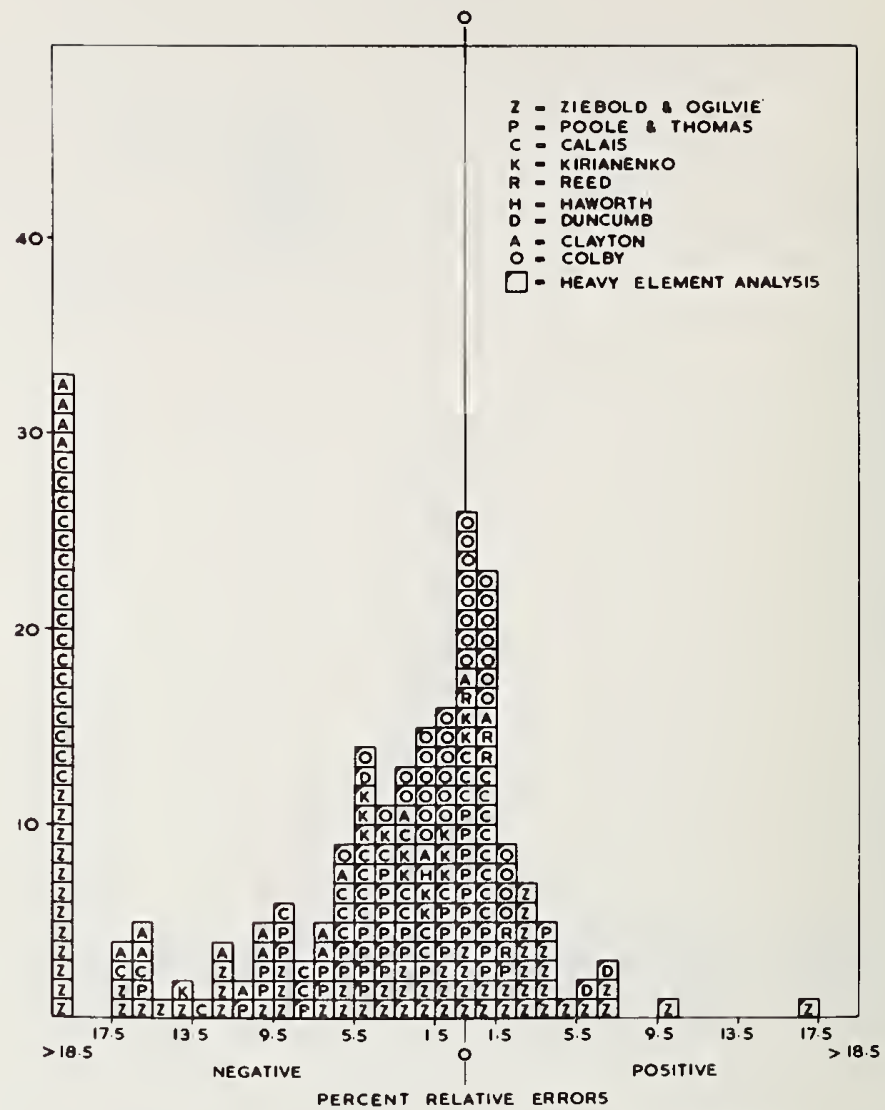

Figure 13. Error Histogram-229 cases, Ziebold method.

The method of Smith [22] though dated 1965 has had little publicity and only recently became widely known. The 150 -case histogram in Figure 11 is of a reasonable shape but shows a strong bias towards negative errors which apparently arises from the method of computing the atomic number correction. Figure 18 compares atomic-number-effect-only calibration curves for the copper-gold system calculated by Smith's method and by the Thomas method via $\alpha$-values, from which it emerges that for both copper and gold analyses the Smith method will give lower values of the corrected concentration; it seems possible that there may be a change with composition from negative to positive errors in certain systems. Thus, it appears that this method is a promising one but 


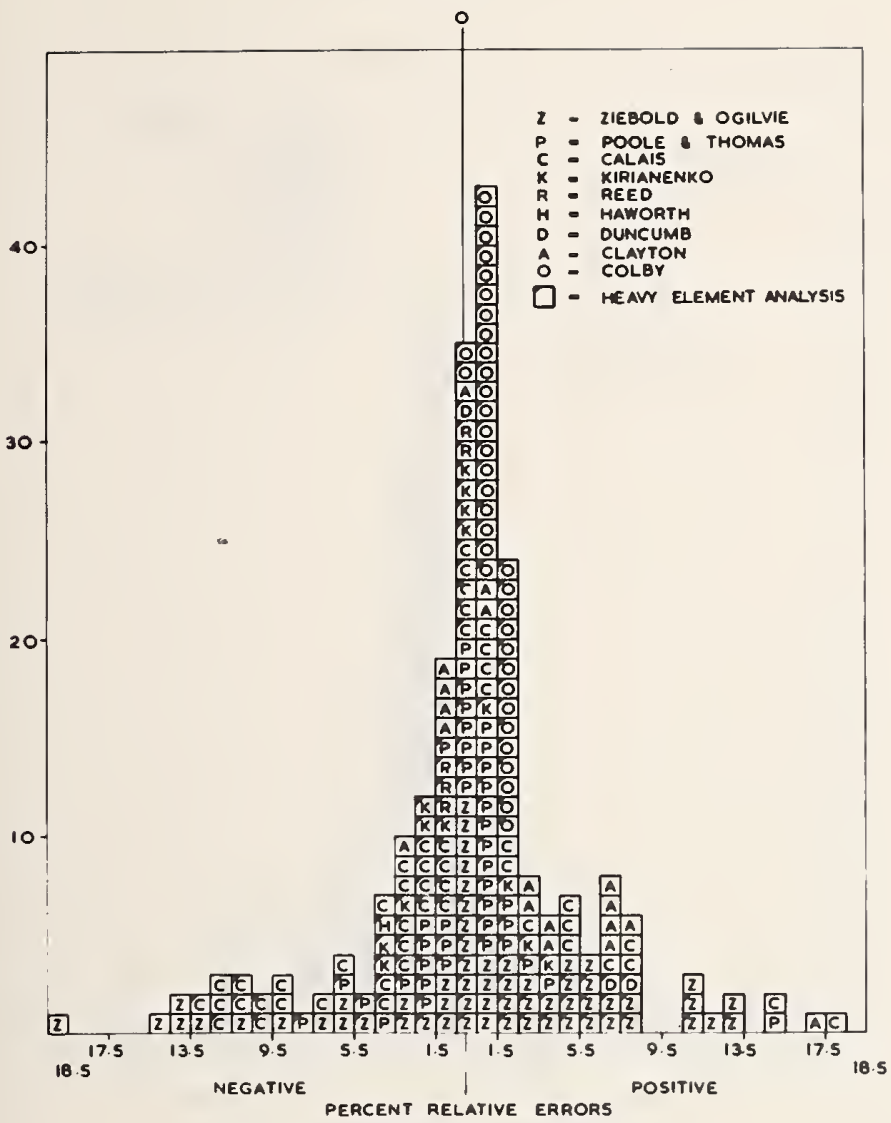

Figure 14. Error Histogram-229 cases, Thomas method.

that in its present form is likely to give rise to a generally low analytical result; of the 150 cases tested here only $30 \%$ fall within the $\pm 2 \frac{1}{2} \%$.

From the foregoing, it is clear that five methods Thomas, Belk, Ziebold, Duncumb, and Smith are the most satisfactory and between them offer either simplicity or accuracy or both. These methods when applied to the 229 cases produced the histograms in Figures 13 to 17 from which emerges a similar pattern of success to that apparent from the 150-case histograms-see also Table 1. The details of the results making up these histograms are given in Appendix II and examination of these and the histograms leads to the following comments. 


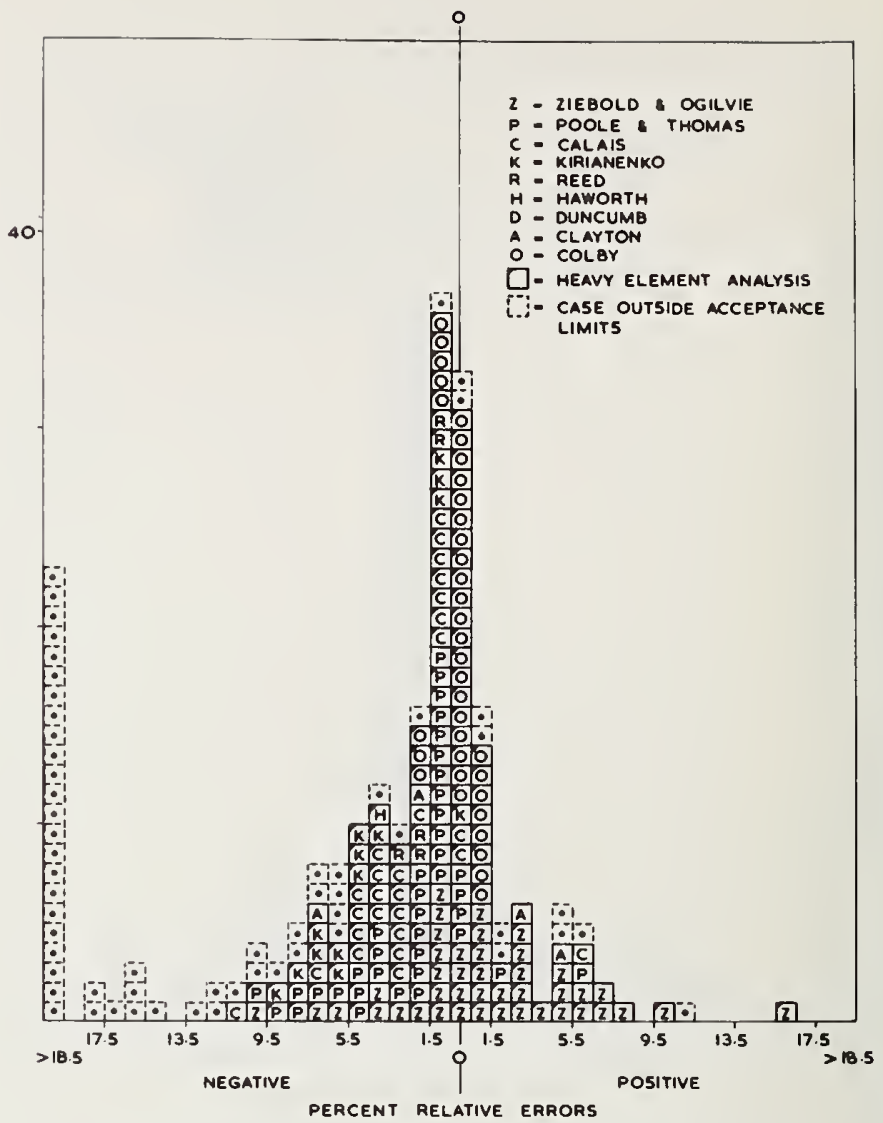

Figure 15. Error Histogram-229 cases, Belk method.

Firstly, the methods of Belk and of Ziebold are quite good when the absorption effects are not large; both methods fail badly on the light element analyses in the data of Ziebold and Ogilvie (e.g., $\mathrm{Ru}-\mathrm{L} \alpha$ in W) and of Calais (e.g., Al- $\mathrm{K} \alpha$ in Ni) especially when the overvoltage is high and the effect of large absorption coefficients most marked. Both methods deal well with results obtained through a wide range of overvoltage when the absorption effects are small, as shown by the measurements of U-L in UC, UN, etc., made by Colby. Both methods are very simple and involve calculations which are quick and easy to do by hand; on the present evidence Belk's method seems slightly more successful. It should be emphasized that neither method is satisfactory when absorption effects are large and reference should be made to Belk's paper for the limits within which he recommends operating. 


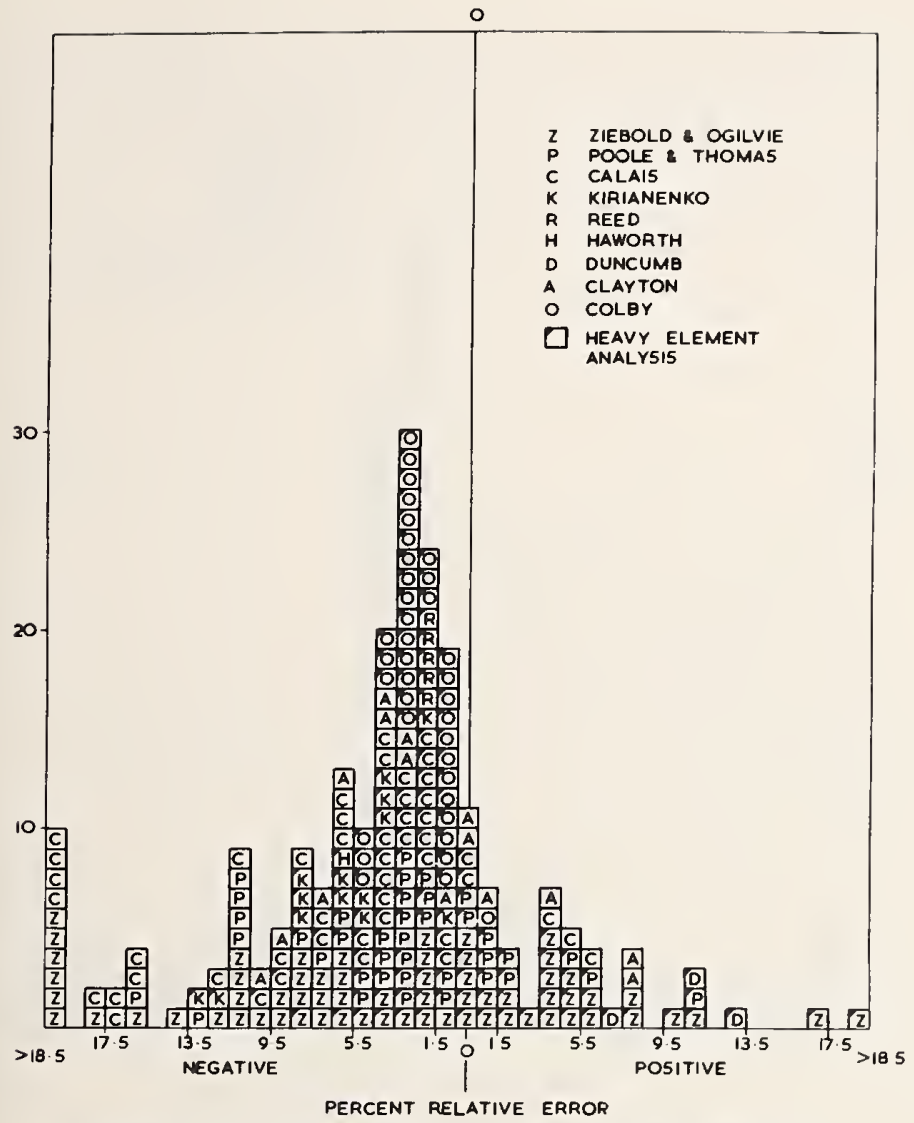

Figure 16. Error Histogram-229 cases, Smith method.

Secondly, the methods of Thomas and Duncumb which are basically similar, give results which are equally satisfactory. Both of them deal tolerably well with the high absorption cases and with results obtained over a wide range of overvoltages. Bearing in mind that there is considerable doubt over the accuracy of the basic data in many cases it does not seem possible to draw any firm conclusions as to the finer shades of merit between these two methods either of which seems to provide a method of general applicability; that of Thomas is a little simpler to carry out by hand but both can readily be computerized.

Thirdly, it now emerges more strongly than before that the Smith method leads to a tendency towards negative errors; the shape of the histogram is quite good but it is centered on about $-3 \%$ relative error and the percentage of results within $\pm 2 \frac{1}{2} \%$ is only $29 \%$. The 


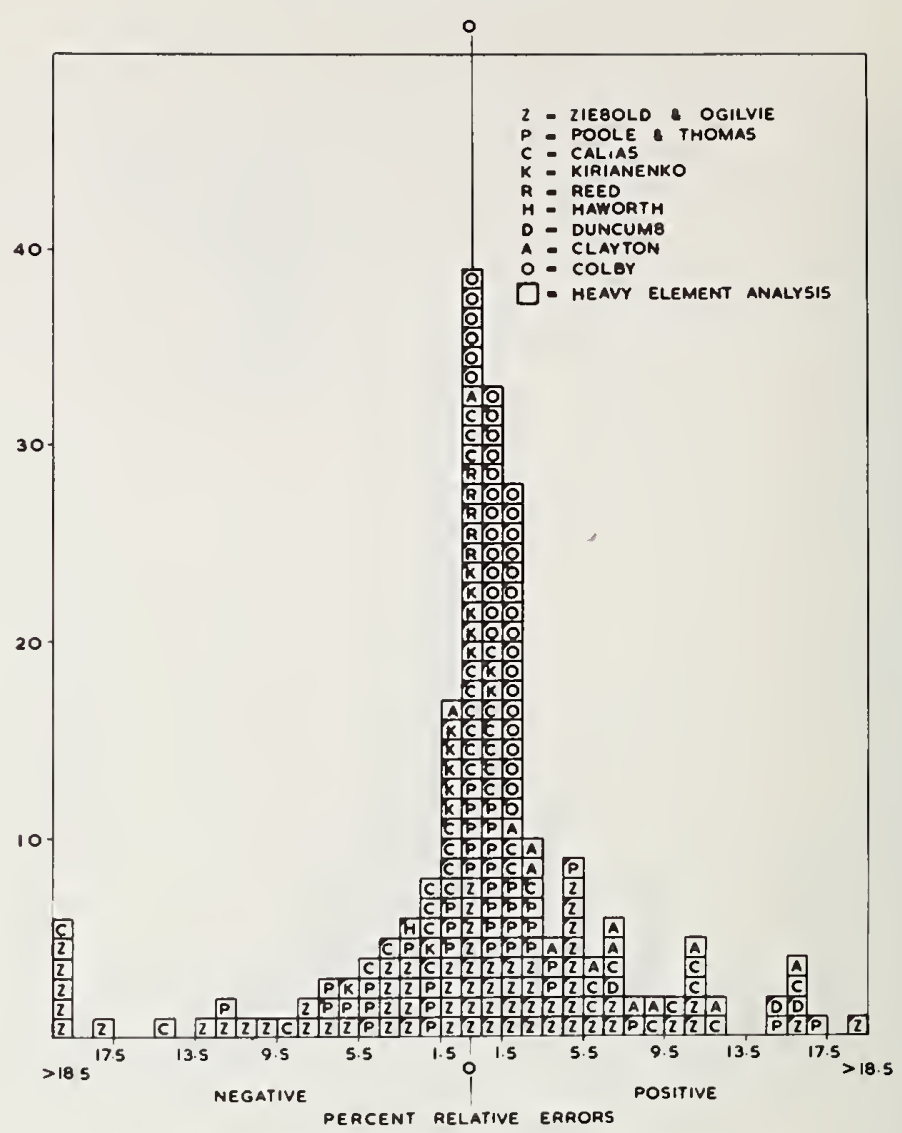

Figure 17. Error Histogram-229 cases, Duncumb method.

appendix notes details of the variation between the absorption coefficients used and those recommended by Smith and it is clear that the overall negative bias in the corrected results does not arise from this factor.

The data employed in this assessment of the various methods fulfill the conditions that the alloy composition should be (supposedly) known, that characteristic fluorescence effects should be negligible, and that atomic number effects should be significant. No other selection has been carried out and the data includes cases where the analyzed element is present in very small amounts, where the "known" compositions may be unreliable, and where very large absorption corrections are involved; these factors no doubt superimpose a considerable spread of errors on top of those arising 
from the correction for the atomic number effect but it is clear that the relative merits of the various procedures can be assessed by a consideration of the overall error histograms. What can not be seen from this treatment, however, is the magnitude of the errors arising solely from the atomic number effect and it is clear that more reliable data are required before the various methods can be more closely compared or refined.

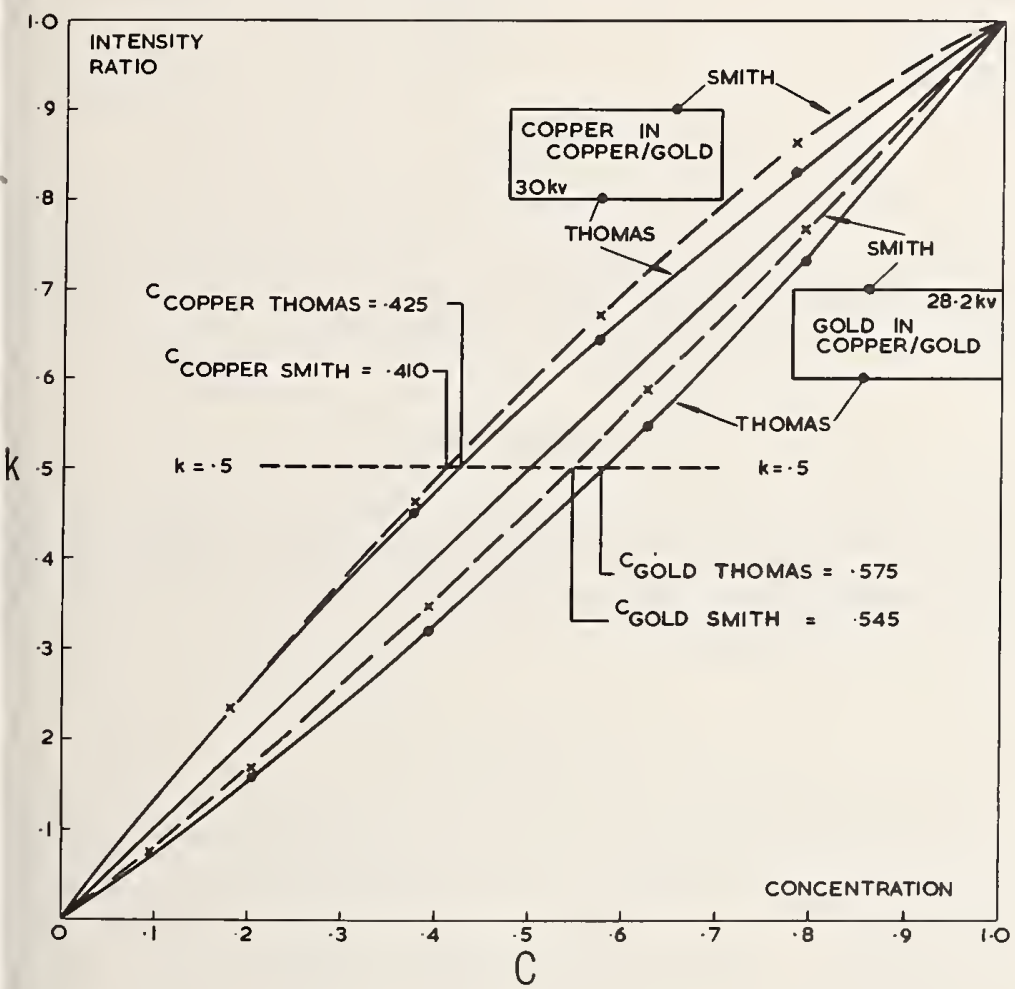

Figure 18. Comparison of Thomas and Smith atomic number correction curves for the copper-gold system (adsorption and fluorescence neglected). 


\section{Table 1}

Method

Archard and Mulvey

Theisen [12]

Theisen [15]

Birks

Ziebold and Ogilvie

Thomas

Belk

Duncumb

Smith
Percentage of results falling within $\pm 21 / 2 \%$ error limits

\section{0 cases}

229 cases

$b(22)$
$b(27)$
$a<27$
$b(13)$
$b 37(44)$
$b 55(53)$
42
53
30

-
-
-
39
61
47
55
29

all 150 cases not computed-performance inferred from smaller sample randomly selected.

' Figures in brackets were obtained when the data was processed by the difference method (see appendix) using early values of absorption coefficients: the remaining figures were obtained by the normalizing method using Heinrich's tabulation of absorption coefficients.

\section{Acknowledgments}

The author wishes to thank most sincerely J. L. Shaw for his patient and extended work in writing the computer programs for the various methods; his contribution formed an essential part of the preparation of this paper.

\section{Note Added in Proof}

Heinrich has recently presented ${ }^{3}$ a discussion of the sources of error in microprobe analysis. In particular, he examines in detail the data used in the present paper and suggests the exclusion of measurements which show inconsistencies, involve low concentrations, or high absorption effects: when this is done the error distribution is greatly improved and Heinrich concludes that the serious errors in the original distributions were due, not to the failure of the atomic number correction, but to poor characterization of samples, high absorption corrections, and fluorescence effects which were not corrected for.

"Common Sources of Error in Electron Probe Microanalysis", K. F. J. Heinrich. Advances in X-Ray Analysis, Vol. 11, Plenum Press, New York, 1967. 


\section{References}

[1] Thomas (1963), B. J. Appl. Phys. 14, p. 397 and AERE-R4593.

[2] Duncumb (1964), 1st European Colloquium on Electron Probe Microanalysis.

[3] Belk (1964), Birmingham C.A.T. Tech. Note MET/26/1964.

[4] Ziebold and Ogilvie (1963), Anal. Chem. 35, p. 621.

[5] Castaing (1960), Advances in Electronics and Electron Physics 13, p. 317.

[6] Archard and Mulvey (1962), Proc. 3rd International Symposium on X-Ray Microanalysis; (1963), B. J. Appl. Phys. 14, p. 626.

[7] Archard (1961), J. Appl. Phys. 32, p. 1505.

[8] Castaing (1951), Thesis to University of Paris and WAL 142/59-7.

[9] Castaing and Descamps (1955), J. Phys. Radium 16, p. 304.

[10] Webster (1931), Phys. Rev. 37, p. 115.

[11] Philibert (1961), J. Inst. Metals 90, p. 241.

[12] Theisen (1961), Euratom Report - I-1.

[13] Tong (1961), unpublished work.

[14] Birks (1963), Electron Probe Microanalysis, Interscience.

[15] Theisen (1965), Quantitative Electron Microprobe Analysis, Springer-Verlag.

[16] Ziebold and Ogilvie (1964), Anal. Chem. 36, p. 322.

[17] Traill and Lachance (1965), Geological Survey of Canada Paper 64-57.

[18] Traill and Lachance (1966), Canadian Spectroscopy 11. Nos. 2 and 3.

[19] Poole and Thomas (1962), J. Inst. Metals 90, p. 241.

[20] Nelms (1958), Supplement, NBS Circular 577.

[21] Il'in and Loseva (1966), Industrial Laboratory 32, (5), p. 664.

[22] Smith (1965), J. of Geology, p. 830.

[23] Green (1962), Proc. 3rd International Symposium on X-Ray Microanalysis, Stanford (Academic Press).

[24] Heinrich (1964), The Electron Microprobe, Wiley and Sons, 1966, p. 296.

[25] Duncumb (1967), private communication.

[26] Bishop (1967), Institute of Physics Conference, London, Feb. 1967.

[27] Poole and Thomas (1964), The Electron Microprobe, Wiley and Sons, 1966, p. 269 and AERE-R4796.

[28] Shaw (1967), Institute of Physics Conference, London, Feb. 1967 and AERER5597. 


\section{Appendix I. Execution of the Methods and Construction of the Histograms}

Two main variations are possible when the correction methods are being used which have an effect on the details of the results; however, it has been established by appropriate comparisons that these effects are only very minor ones and do not significantly influence the overall picture of the value of the various methods. These variations are:

(a) Choice of absorption coefficients. The tabulation by Heinrich (1964) [24] has been preferred in this work but certain of the histograms are based on earlier values (see Poole and Thomas 1964) [27]; the Thomas method has been carried through using both the early and Heinrich values and no great difference was observed.

(b) Treatment of binary alloys. Where only one component has been measured the other has to be estimated by difference in order to calculate alloy absorption coefficients and other parameters. Where both components have been measured then each can be dealt with entirely separately, as in the former circumstance (the difference approach; alternatively, best values for both components can be normalized and used for estimating alloy absorption coefficients etc., at each stage of iterative procedures (the normalizing approach). Hand calculation using the difference approach was employed on the methods of Archard and Mulvey, Theisen and Birks; computer calculation ${ }^{1}$ using the normalizing approach was employed on the iterative methods of Thomas, Belk, Smith, and Duncumb; computer calculation was also used on Ziebold and Ogilvie although this does not involve estimating composition dependent parameters or iterations.

Relative errors were calculated for each case by comparing the corrected data to the "known" concentrations and used in the construction of the histograms; a low result is recorded as a negative error.

1 The computer programs were written by Shaw and were based on his universal microanalysis correction program (Shaw 1967) [28] which can deal with multicomponent systems. 


\begin{tabular}{|c|c|c|}
\hline \multirow{6}{*}{ 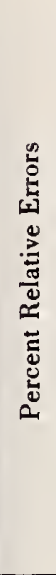 } & $\rightarrow$ & ーーーー \\
\hline & 吾 & 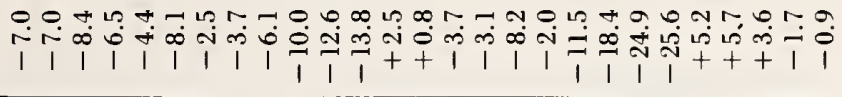 \\
\hline & 㤩茝 & $\begin{array}{l}\infty \\
0 \\
0\end{array}$ \\
\hline & 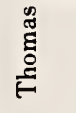 & 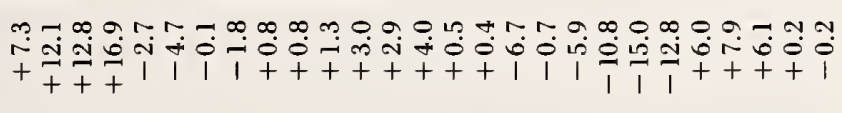 \\
\hline & 竞 & 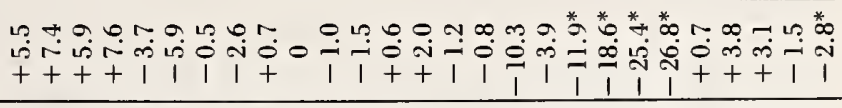 \\
\hline & $\begin{array}{l}0 \\
\infty \\
N\end{array}$ & 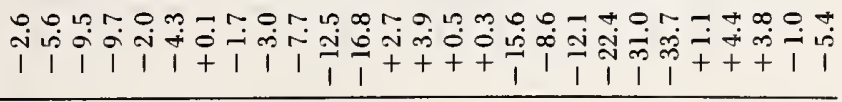 \\
\hline \multicolumn{2}{|c|}{10} & 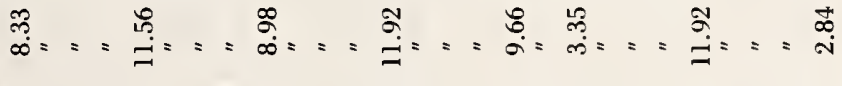 \\
\hline \multicolumn{2}{|c|}{$\overbrace{1}^{\infty}$} & 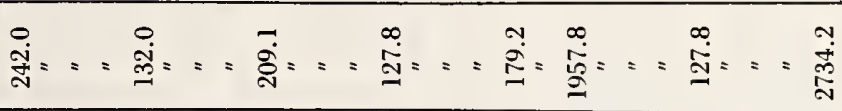 \\
\hline \multicolumn{2}{|c|}{$\overbrace{1}^{4}$} & 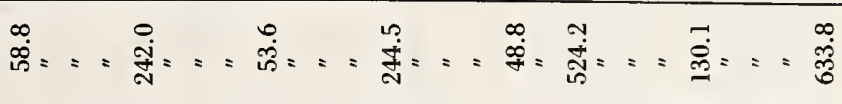 \\
\hline \multicolumn{2}{|c|}{ 递苛官 } & 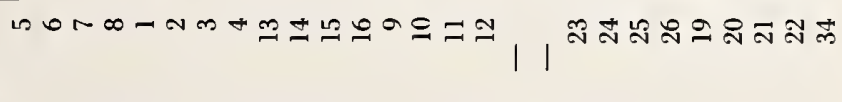 \\
\hline \multicolumn{2}{|c|}{ 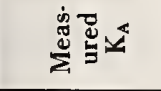 } & 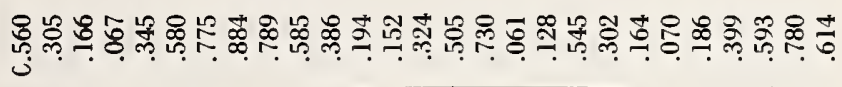 \\
\hline \multicolumn{2}{|c|}{$\begin{array}{l}0 \\
0 \\
\dot{0} \\
\dot{D} \\
0\end{array}$} & 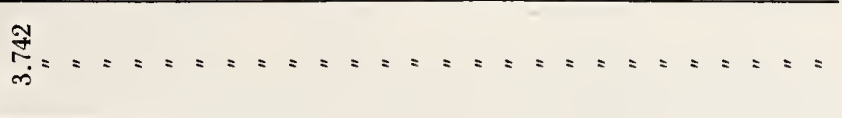 \\
\hline \multicolumn{2}{|c|}{$\geq$} & ㅇ: = = = = = = = = = = = = = = = = = = = = = = \\
\hline \multicolumn{2}{|c|}{ 蛋草 } & 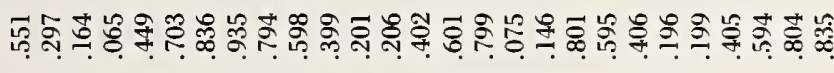 \\
\hline \multicolumn{2}{|c|}{ 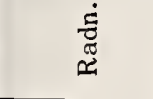 } & 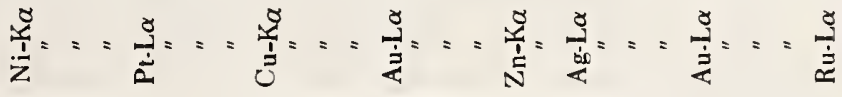 \\
\hline \multicolumn{2}{|c|}{ 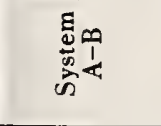 } & 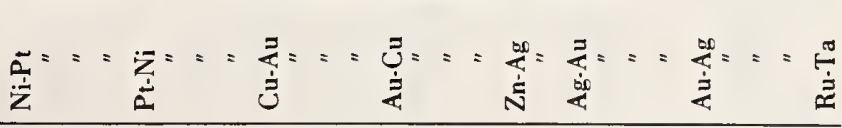 \\
\hline \multicolumn{2}{|c|}{ 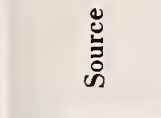 } & 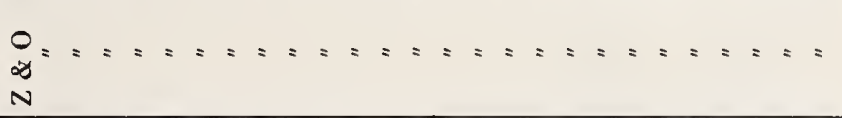 \\
\hline \multicolumn{2}{|c|}{$\dot{\dot{z}}$} & 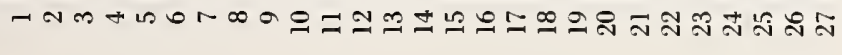 \\
\hline
\end{tabular}




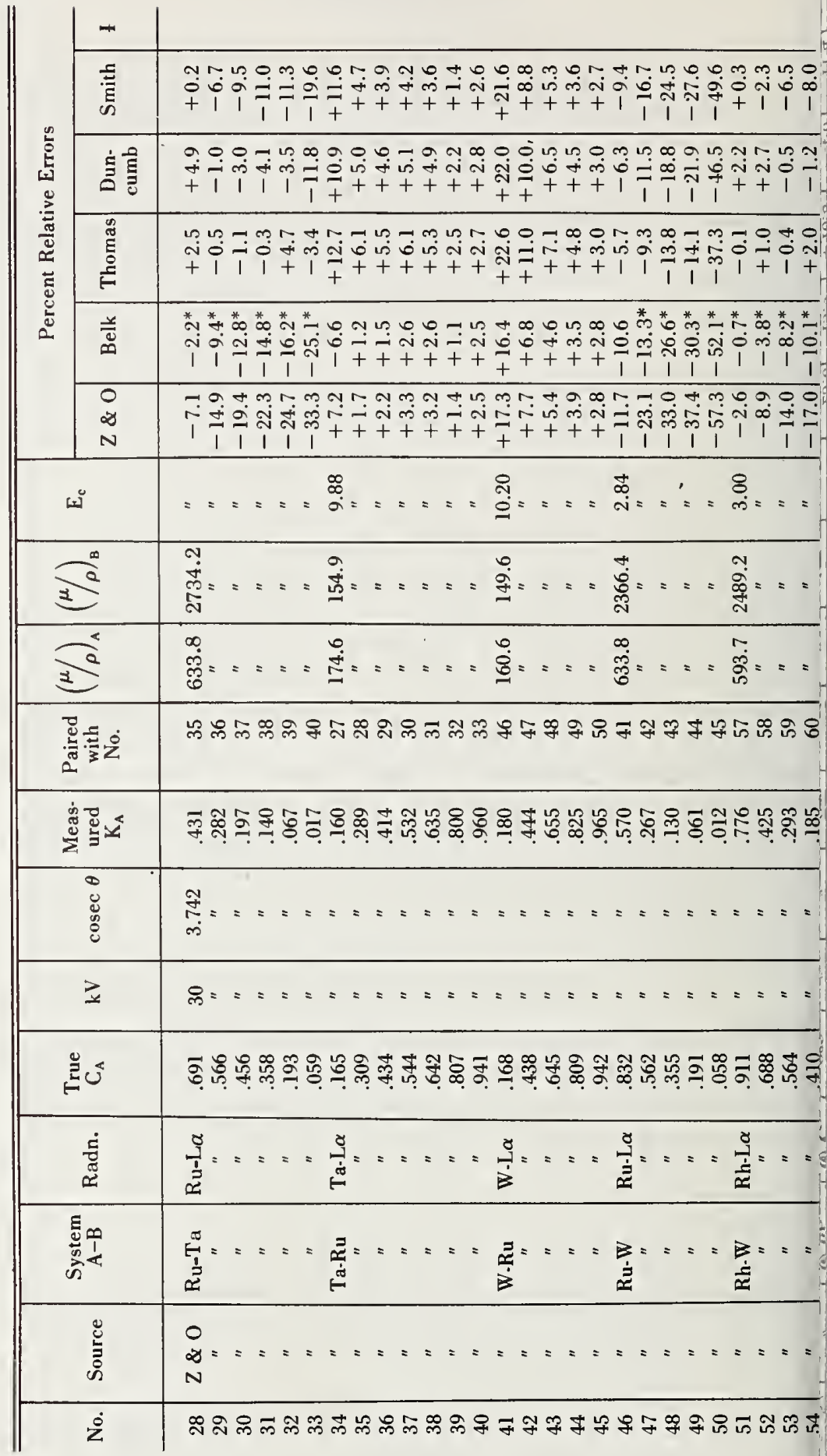




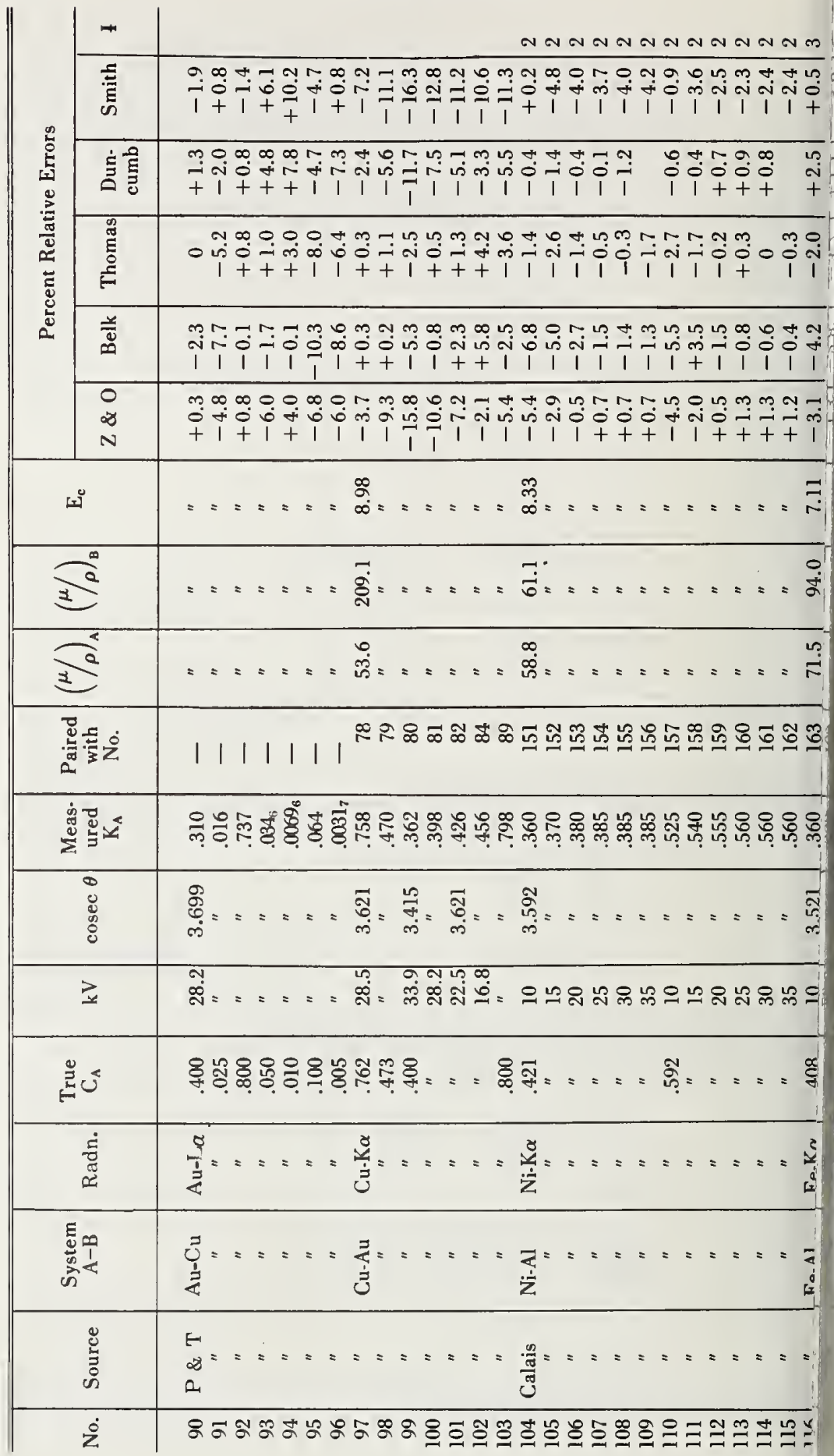




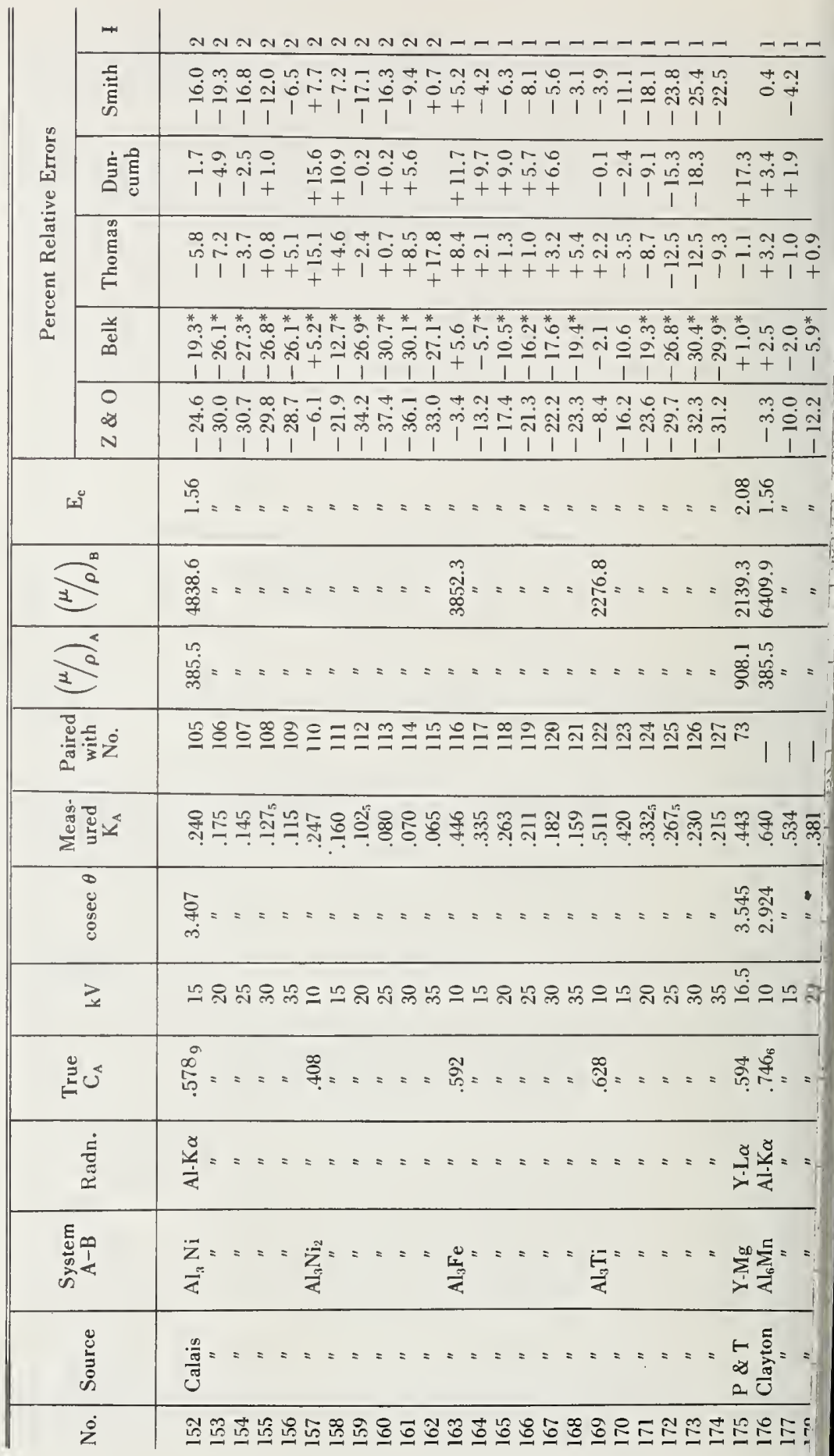




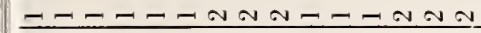

0

\begin{tabular}{|c|c|c|}
\hline 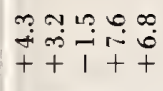 & 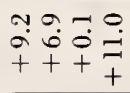 & 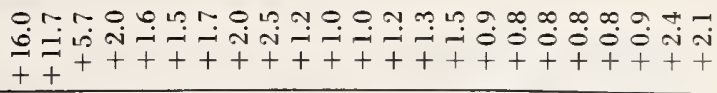 \\
\hline
\end{tabular}

ดกทกด ๓ं

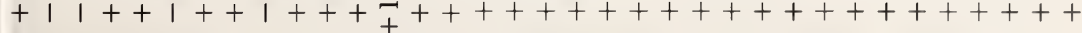

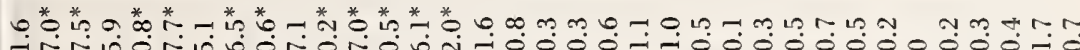

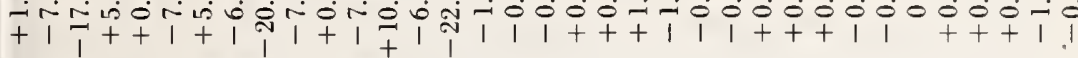

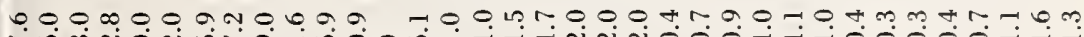


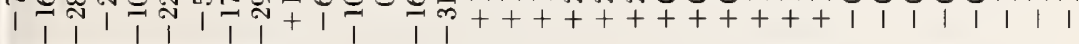

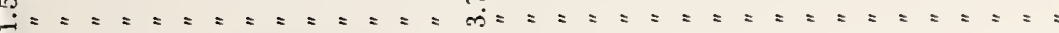

\begin{tabular}{|c|c|c|c|c|c|c|c|c|c|c|c|c|c|c|c|c|c|c|c|c|c|c|c|}
\hline$\infty_{\infty}^{10}=$ & $=\frac{m}{\infty}=$ & $=$ & $\begin{array}{l}0 \\
\infty \\
\underset{\infty}{\infty} \\
\underset{+}{\infty}\end{array}=$ & $=$ & $\begin{array}{l}0 \\
\frac{0}{2} \\
\frac{3}{6}\end{array}$ & $=$ & $\begin{array}{l}\tilde{n} \\
\dot{m} \\
\dot{m}\end{array}$ & $=$ & $\ddot{\circ}=$ & $=$ & $=$ & $=$ & $=$ & $\stackrel{\leftrightarrow}{\circ}=$ & $=$ & $=$ & $=$ & $=$ & $\ddot{\ddot{g}}=$ & $=$ & $=$ & $=$ & $=\frac{\varphi}{\dot{0}}=$ \\
\hline
\end{tabular}

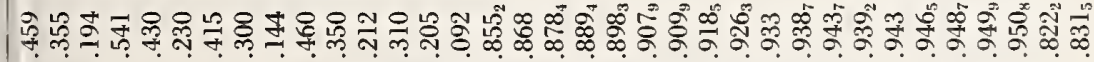

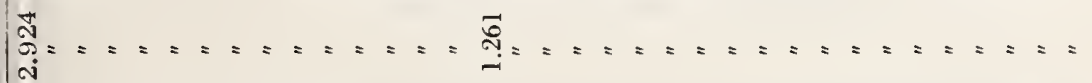

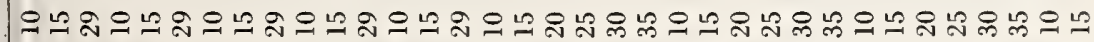

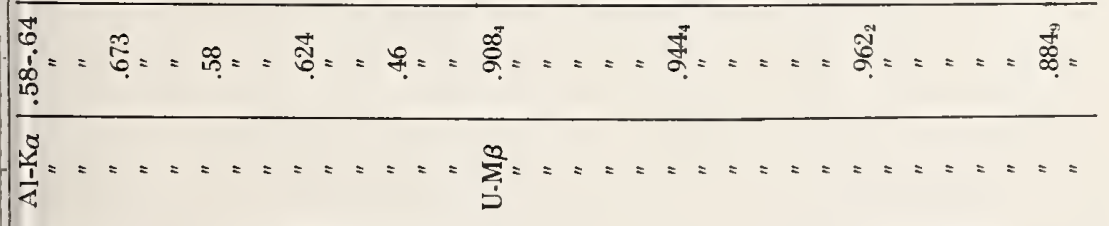

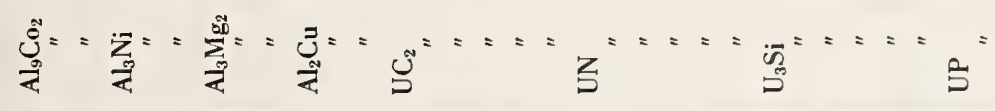

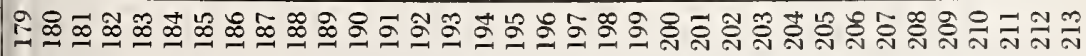




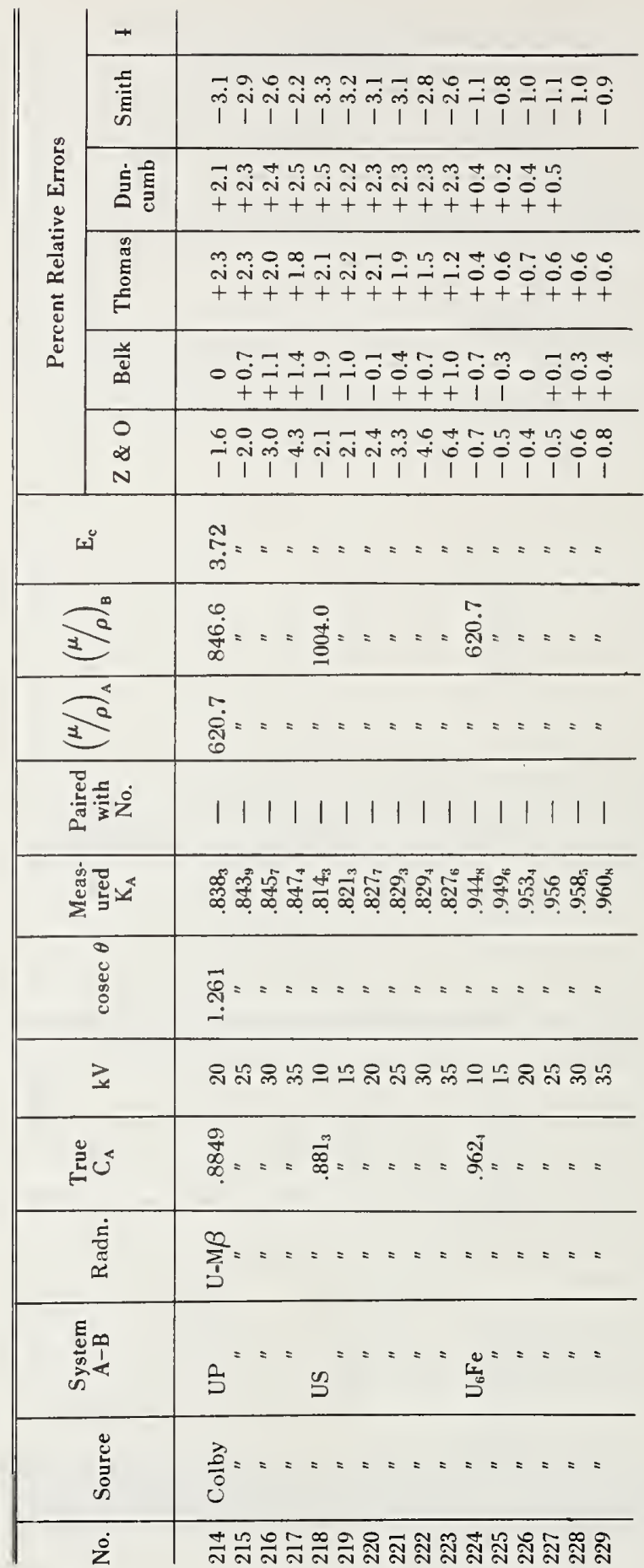


Key:

* Case outside Belk's 2\% accuracy absorption limits.

I Numbers in this column indicate the variation between the absorption coefficients used in compiling the table of errors for Smith's method and the coefficients recommended by Smith; the code used is as follows:

No entry-coefficients the same

1 - either or both different from Smith's values by up to $2 \%$

2 - either or both different from Smith's values by $2 \%-5 \%$

3 - either or both different from Smith's values by $5 \%-10 \%$

4 - either or both different from Smith's values by greater than $10 \%$

Prime-magnitude of coefficients very small

$\neq$ Kirianenko 



\section{THE CALCULATION OF STOPPING POWER AND BACKSCATTER EFFECTS IN ELECTRON PROBE MICROANALYSIS}

\section{P. DUNCUMB}

Tube Investments Research Laboratories, Hinxton Hall, Cambridge, England

and

\section{S. J. B. REED}

Mineralogy Department, British Museum (Natural History), Cromwell Road, London, S.W.7

\section{Abstract}

The problem of calculating backscatter factors and stopping power for atomic number corrections in quantitative microprobe analysis is discussed. It is concluded that the stopping power $\mathrm{S}$ is best calculated from Bethe's formula, using values of the mean ionization potential $\mathbf{J}$ determined empirically from analyses of known compounds. A table of $\mathbf{J}$ values for practical calculations is given. Values of the backscatter factor $\mathrm{R}$ are also given, from calculations using Bishop's experimental measurements of electron backscattering coefficients and energy distributions. Simplifications to the theory are discussed, together with methods of averaging $\mathrm{S}$ and $\mathrm{R}$ for compounds. The accuracy and range of application of the atomic number correction are considered, and areas where further experimental work might lead to useful improvements are indicated.

\section{Introduction}

It was demonstrated by Castaing [1] that the relationship between the mass concentration $\mathrm{C}_{A}$ of an element $\mathrm{A}$ in a specimen analyzed in the microprobe, and the ratio $\mathrm{k}_{A}$ of the intensity of the characteristic $\mathrm{x}$-ray line (usually $\mathrm{K} \alpha$ ) from the specimen to that from a standard consisting of the pure element $\mathrm{A}$, is, to a first approximation, simply

$$
\mathrm{k}_{A}=\mathrm{C}_{A}
$$

It is assumed that instrumental corrections, such as those for deadtime and background are carried out before calculating $\mathrm{k}_{A}$, and that the intensities from specimen and standard are measured under 
identical experimental conditions. Departures from the simple law of proportionality expressed in equation (1) can be large, and if accurate quantitative analysis is to be attempted with the microprobe, it is essential to apply a correction factor

$$
\mathrm{k}_{A}=\mathrm{C}_{A} \times \text { (correction factor) }
$$

The correction factor is placed on the right hand side of the equation because it is itself a function of $C_{A}$, and of the concentrations of the other elements present.

Following Castaing, most authors have divided the total correction factor into three separate factors: absorption, fluorescence, and atomic number. Methods of calculating correction factors for the absorption of the characteristic x-rays in emerging from the specimen, and for the additional intensity contributed by fluorescence excited by other $\mathrm{x}$-rays in the spectrum, were proposed by Castaing and have been developed further by many authors. The third correction, the so-called "atomic number correction", takes into account the non-linearity in the relationship between $\mathrm{k}_{A}$ and $\mathrm{C}_{A}$ which remains after the other two corrections have been applied. This might more appropriately be called the "generation correction" since it is governed by the relationship between the intensity of characteristic $x$-rays generated in a specimen and its composition. The term atomic number correction has come to be used because the properties of an element with respect to x-ray generation are largely determined by its atomic number.

In the microprobe, electrons accelerated to an energy in the range $5-40 \mathrm{keV}$ are focused into a probe, usually of the order of $1 \mu \mathrm{m}$, or rather less in diameter, carrying a current generally between $10^{-6} \mathrm{~A}$ and $10^{-9} \mathrm{~A}$, which is made to strike the specimen at the point to be analyzed. The incident electrons are subject to interactions with the atoms in the specimen. These are broadly classified into elastic scattering in which there is a considerable change of direction, but negligible loss of energy, and inelastic scattering characterized by significant energy loss. Much of the high-angle elastic scattering is the result of interactions with atomic nuclei, whereas inelastic scattering is due to interactions with atomic electrons. The spatial distribution of incident electrons in the specimen is largely governed by elastic scattering, while inelastic scattering determines their deceleration and how far they travel before coming to rest. One form of inelastic interaction is ionization of an inner electron shell, such 
as the $\mathrm{K}$ or $\mathrm{L}$ shell, which is followed in a certain proportion of cases by the production of a $\mathrm{K}$ or $\mathrm{L}$ characteristic x-ray quantum. However, an inner shell ionization is a relatively rare event, and the main contribution to the stopping of incident electrons is from large numbers of inelastic interactions with outer atomic electrons. Each electron suffers multiple scattering before either finally coming to rest in the specimen or leaving the specimen altogether, as shown in Figure 1. The physical picture is thus one of considerable complexity, which makes the atomic number correction the most difficult of the three major corrections to reduce to a general and reasonably simple form.

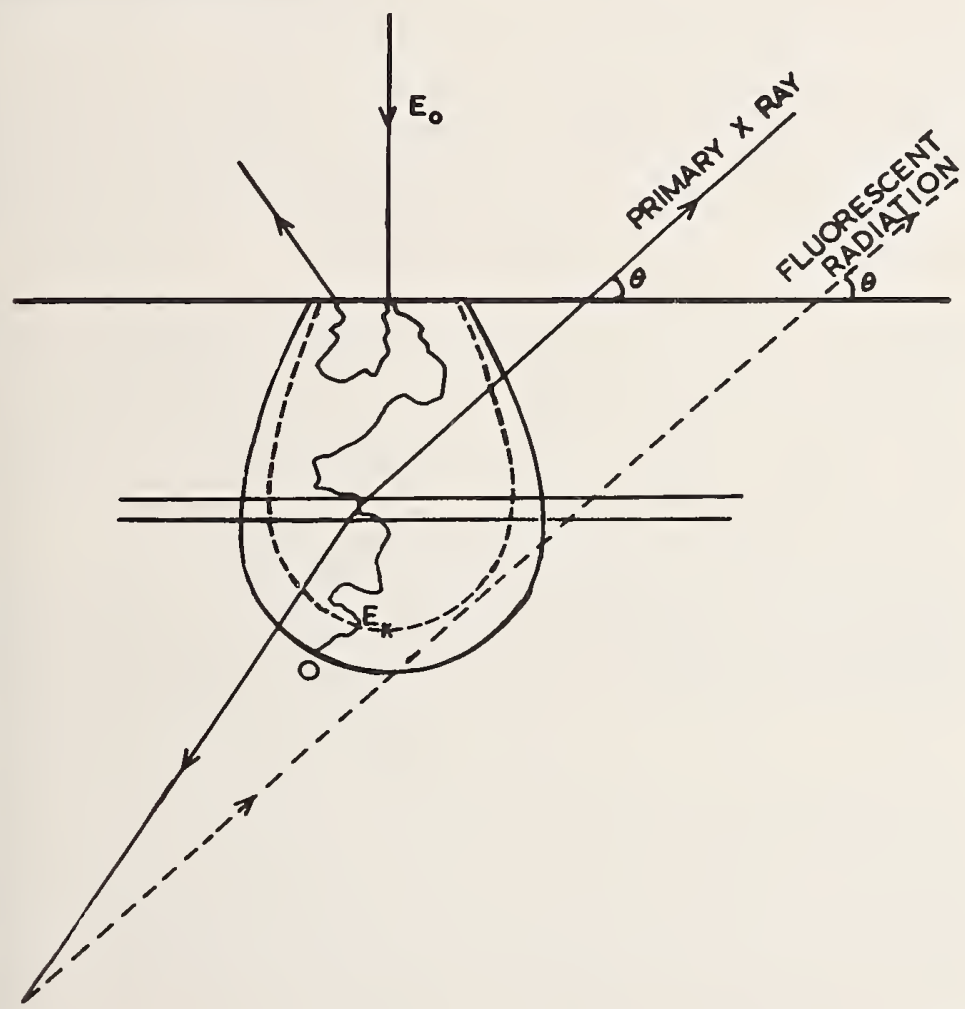

Figure 1. Schematic representation of the volume irradiated by electrons incident on the target. Characteristic emission may be generated anywhere along the track of an electron, up to the point where its energy has fallen to the critical excitation potential $\mathbf{E}_{k}$. Some electrons are backscattered from the surface and cause reduction in the mean ionization per electron. 
The ionization cross-section, $\mathrm{Q}$, is defined as the probability per unit path length of an electron with a given energy causing ionization of a particular inner electron shell $(\mathrm{K}, \mathrm{L}$, or $\mathrm{M})$ of an atom in the specimen. There is enough experimental information to establish with reasonable accuracy the dependence of $\mathrm{Q}$ on electron energy, E. It is therefore possible to calculate characteristic $x$-ray intensities, given the number and energy distribution of electrons in the specimen. For this purpose it is necessary to have a model of the physical situation which is amenable to mathematical treatment. All such models involve a degree of simplification which makes it essential to have experimental confirmation of results. The relatively simple diffusion model of Archard [2] is not sufficiently close to physical reality to be entirely reliable as a basis for calculating atomic number corrections. More sophisticated calculations by Brown [3], based on a Boltzmann transport equation adapted to electrons, give $\mathrm{x}$-ray absorption factors which agree quite well with experimental data, but fail to reproduce accurately the observed energy distribution of backscattered electrons. The most physically satisfactory approach is the use of basic electron scattering data in a computer program which synthesises electron trajectories by a Monte Carlo procedure. Green [4] and Bishop [5] have used this method to compute various quantities, such as electron backscattering factors and x-ray depth distributions, some of which can be measured experimentally. The agreement with experiment obtained still falls short of that required for atomic number corrections, owing partly to inadequate scattering data, but also because, even with the largest contemporary computers, it is not possible to simulate physical reality with sufficient accuracy. In any case it would not be practicable to carry out a Monte Carlo calculation for every analysis to be corrected. Even in the Monte Carlo method, it has proved necessary to make use of a continuous mathematical function to represent the loss of energy by an electron as it travels through a specimen, whereas energy is in fact lost in discrete amounts, and the energy lost by electrons travelling a given distance is variable.

Given a suitable equation for energy loss per unit distance together with an expression for $\mathrm{Q}$, the intensity of $\mathrm{x}$-rays which would be generated if all the incident electrons stayed in the specimen can readily be calculated, independently of any model or other assumption. This procedure ignores the statistical nature of energy loss by inelastic scattering, but this is equally true of the models discussed above, which are chiefly of value for the 
information they give about backscattering. There is in addition a considerable amount of experimental data on electron backscattering, and it is preferable in practice to make use of this rather than data obtained from theoretical models, although the latter are useful, for instance, in suggesting how to deal with backscattering from compounds.

The ability of a given material to slow down incident electrons is usually given in terms of stopping power $\mathrm{S}$ defined by

$$
\mathrm{S}=-(1 / \rho) \cdot(\mathrm{dE} / \mathrm{dx})
$$

where $\rho$ is the density of the material, $\mathrm{x}$ is distance measured along the path, and the incremental energy change $\mathrm{dE}$ refers to the mean change of energy in travelling a distance $\mathrm{dx}$. Stopping power defined in this way is approximately constant for a particular chemical element regardless of its physical or chemical state. It follows from the additive nature of energy losses that the stopping power for a compound specimen $\mathrm{AB}$ is given by

$$
\mathrm{S}_{A B}=\Sigma_{i} \mathrm{C}_{i} \mathrm{~S}_{i}
$$

where $C_{i}$ is the mass concentration of the $i^{\text {th }}$ element, the stopping power of which is $S_{i}$.

The most suitable formula for stopping power is that given by Bethe [6],

$$
\mathrm{S}=\text { const. }(\mathrm{Z} / \mathrm{A}) \cdot(1 / \mathrm{E}) \ln (1.166 \mathrm{E} / \mathrm{J})
$$

where $\mathrm{Z}$ and $\mathrm{A}$ are respectively the atomic number and atomic weight of the element concerned. The constant 1.166 is correct in this case, rather than the value 2 often encountered in the literature, which is appropriate when the incident particles are protons or other positive ions, (Bethe and Ashkin [7]).

Bethe's formula was originally derived for the hydrogen atom, and the extension to heavier atoms necessitated the introduction of the mean ionization potential, $\mathbf{J}$, which must be expressed in the same units as the electron energy $E$ in equation (5). Bloch [8] deduced from the Thomas-Fermi statistical model of the atom that $\mathbf{J}$ should be directly proportional to $\mathrm{Z}$ making $\mathrm{J} / \mathrm{Z}$ constant, with a value of around $13.5 \mathrm{eV}$. Several experimental investigations have confirmed that this is at least approximately true, though the energies at which the experiments were carried out were generally much higher than those encountered in the microprobe, and agreement between different experimenters is not very good. Wilson [9] obtained a value $\mathrm{J} / \mathrm{Z}=11.5 \mathrm{eV}$ for aluminium which has been 
widely used in the literature, and may reasonably be substituted in equation (5), if it is to be assumed that $J / Z$ is constant. For some purposes this assumption does not lead to any significant error, but we conclude in a later section that it is necessary to use a somewhat different relation between $\mathbf{J}$ and $\mathbf{Z}$.

From the definition of the ionization cross-section, Q, it follows that the average number $\mathrm{n}$ of $\mathrm{K}$ ionizations per electron incident with energy $\mathrm{E}_{o}$ is given by

$$
\mathrm{n}=\left(\mathrm{N}_{\circ} \rho \mathrm{C}_{A} / \mathrm{A}\right) \int_{\mathrm{E}_{\circ}}^{\mathrm{E}_{K}} \frac{\mathrm{Q}}{\mathrm{dE} / \mathrm{dx}} \cdot \mathrm{dE}
$$

where $\mathrm{N}_{o}$ is Avogadro's number, $\mathrm{C}_{A}$ is the mass concentration of element $A$, and $E_{K}$ is its critical $K$ excitation potential. The same formula applies to lines other than $\mathrm{K}$, provided the appropriate $\mathrm{Q}$ function and excitation potential are substituted. Making use of equation (3), equation (6) becomes

$$
\mathrm{n}=\left(\mathrm{N}_{o} \cdot \mathrm{C}_{A} / \mathrm{A}\right) \int_{\mathrm{E}_{K}}^{\mathrm{E}_{\mathrm{o}}}(\mathrm{Q} / \mathrm{S}) \mathrm{dE}
$$

The number $\mathrm{n}$ of $\mathrm{K}$ ionizations is directly proportional to the intensity of the $\mathrm{K} \alpha$ line which is measured in the analysis, but in calculating this intensity, backscattering must be taken into account. This is achieved by introducing a factor $\mathbf{R}$, which is equal to the ratio of the intensity actually generated to that which would be generated if all the incident electrons stayed in the specimen, and is always less than unity. We thus arrive at a formula for the intensity of characteristic $\mathrm{x}$-rays generated

$$
\mathrm{I}_{A}=\text { const. } \mathrm{C}_{A} \cdot \mathrm{R} \int_{\mathrm{E}_{K}}^{\mathrm{E}_{\mathrm{o}}}(\mathrm{Q} / \mathrm{S}) \mathrm{dE}
$$

\section{Evaluation of the Backscatter Coefficient $\mathbf{R}$}

The backscatter coefficient $\mathrm{R}$ is related to the electron backscatter coefficient $\eta$ which is the fraction of the incident electrons which are backscattered. Figure 2 shows values of $\eta$ measured by Bishop [10] for an electron beam of energy $E_{o}=20 \mathrm{keV}$ incident normally on the specimen. Similar results have been obtained by other workers [11], and it is well established that $\eta$ is essentially a smoothly varying function of $Z$, and is almost independent of $E_{o}$. in the range with which we are concerned. If all backscattered electrons retained their original energy $E_{o}$, then $1-R$ would be equal to 


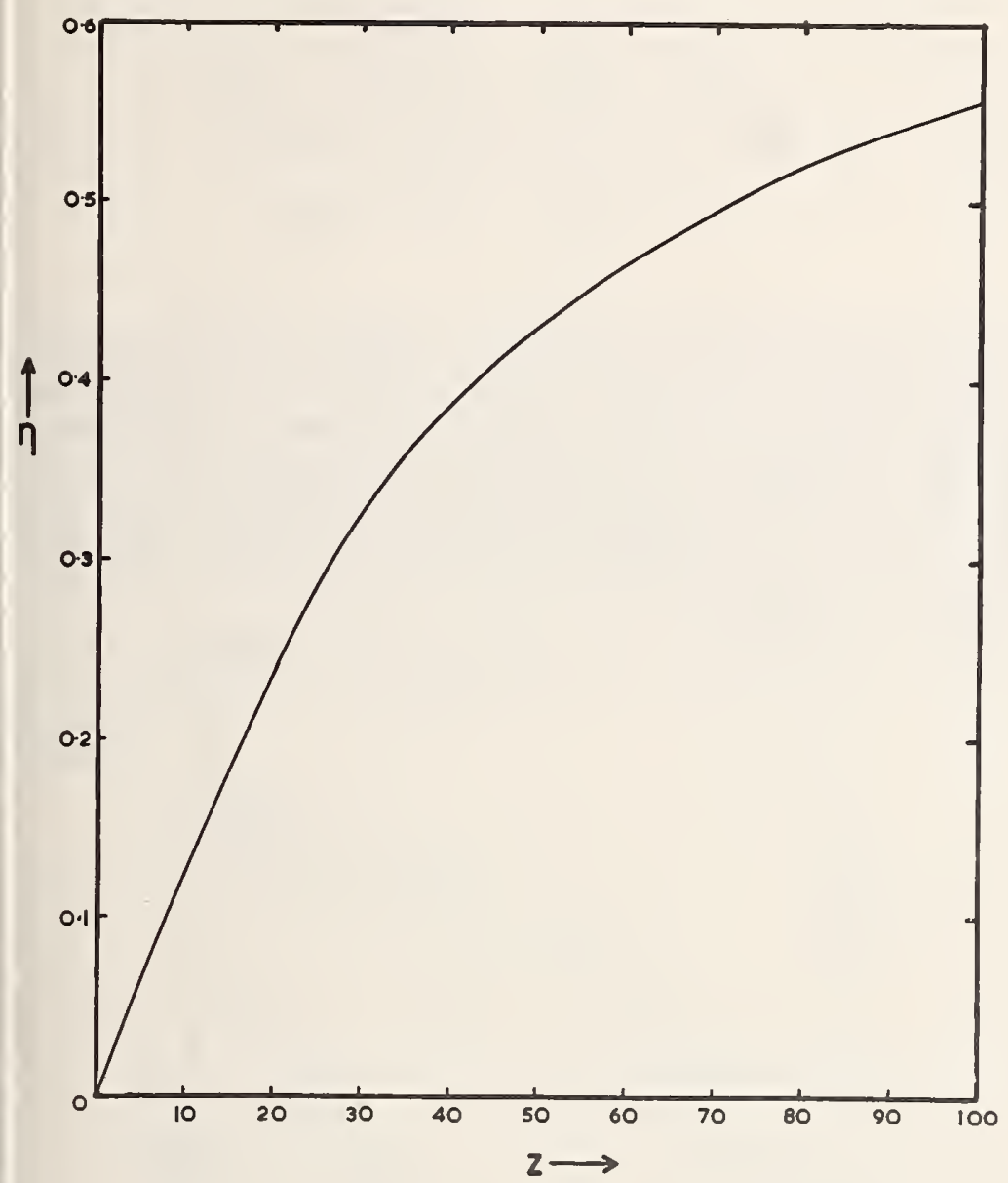

Figure 2. Fraction of electrons backscattered (with incident beam normal to surface) as a function of target atomic number (Bishop [10]).

$\eta$. In fact they possess a range of energies, from $\mathrm{E}_{o}$ down, which makes $1-R$ less than $\eta$ by an amount which depends on the energy distribution of the backscattered electrons.

Webster [12] showed that R may be calculated from the energy distribution of backscattered electrons, given expressions for $\mathrm{Q}$ and S. Alternative methods of calculation proposed by Thomas [13] and Tomlin [14] are not completely rigorous. Following Webster, therefore, $\mathrm{R}$ is calculated from the formula 


$$
1-R=\frac{\int_{W_{K}}^{1} \frac{d \eta}{d W} \int_{E_{K}}^{W E_{o}} \frac{Q}{S} d E_{\cdot} d W}{\int_{E_{K}}^{E_{o}} \frac{Q}{S} d E}
$$

where the new variable $\mathrm{W}$ is equal to $\mathrm{E} / \mathrm{E}_{0}$. The backscattered electron energy distribution is represented by the differential $\mathrm{d} \eta / \mathrm{dW}$. Experimental curves of $\mathrm{d} \eta / \mathrm{dW}$ for carbon, copper, silver, and gold, due to Bishop [10], are shown in Figure 3(a). It is found experimentally that, not only is $\eta$ insensitive to beam energy $\mathrm{E}_{0}$, but also the shape of the distribution expressed as a function of $W$ is also largely independent of $\mathrm{E}_{o}$.

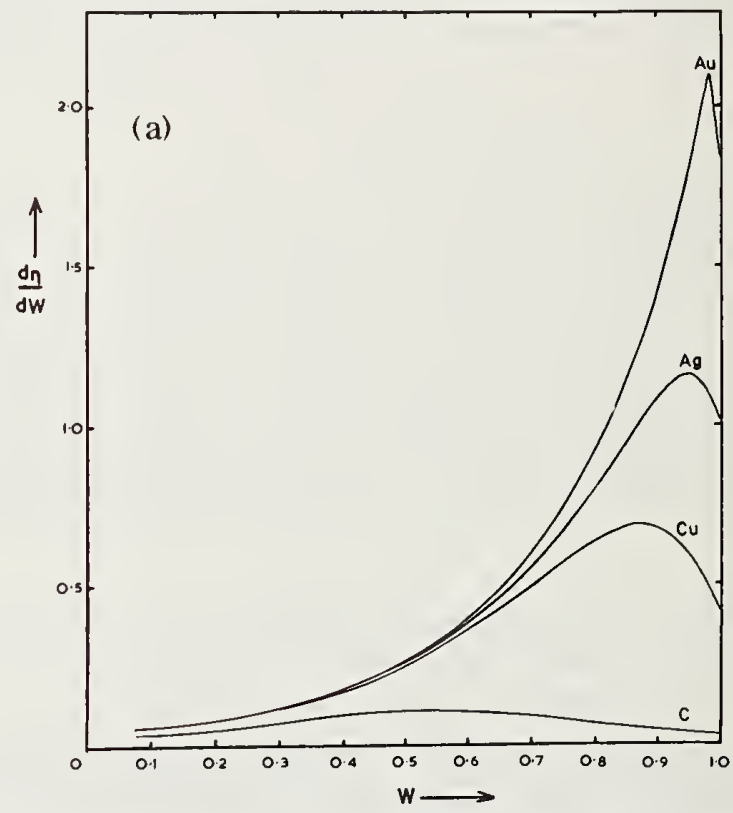

Figure 3(a). Energy distribution of backscattered electrons (normal incidence), measured by Bishop [10], for carbon, copper, silver, and gold.

The backscattered electron energy distribution may alternatively be expressed in integral form as $\eta(W)$, the number of backscattered electrons with energy greater than $\mathrm{WE}_{0}$, which results in a rather simpler formula for $\mathbf{R}$ 


$$
1-\mathrm{R}=\frac{\int_{\mathrm{W}_{\mathrm{K}}}^{1} \eta(\mathrm{W}) \frac{\mathrm{Q}}{\mathrm{S}} \mathrm{dW}}{\int_{\mathrm{E}_{\mathrm{K}}}^{\mathrm{E}_{\mathrm{o}}} \frac{\mathrm{Q}}{\mathrm{S}} \mathrm{dE}}
$$

$\mathrm{R}$ has been calculated from equation (10) using Bishop's data for $\eta(\mathrm{W})$ normalized to values of $\eta$ from Figure 2 and interpolated smoothly for elements between those for which the distribution was experimentally determined (see Figure 3(b)). Equation (5) was used for $\mathrm{S}$, assuming $\mathrm{J} / \mathrm{Z}=11.5 \mathrm{eV}$, with the formula for $\mathrm{Q}$ given by Webster et al. [12]. Variation of $\mathrm{J} / \mathrm{Z}$ within the limits of uncertainty which exist for this quantity has a negligible effect on R. Similarly the dependence of $\mathrm{R}$ on $\mathrm{E}_{\boldsymbol{o}}$ arising from the variation of $\mathrm{S}$ with $\mathrm{E}$ is very slight and may be neglected, so that $R$ may be expressed as a function of $\mathrm{Z}$ and overvoltage ratio $\mathrm{U}=\mathrm{E}_{o} / \mathrm{E}_{K}$ only, as shown in Figure 4.

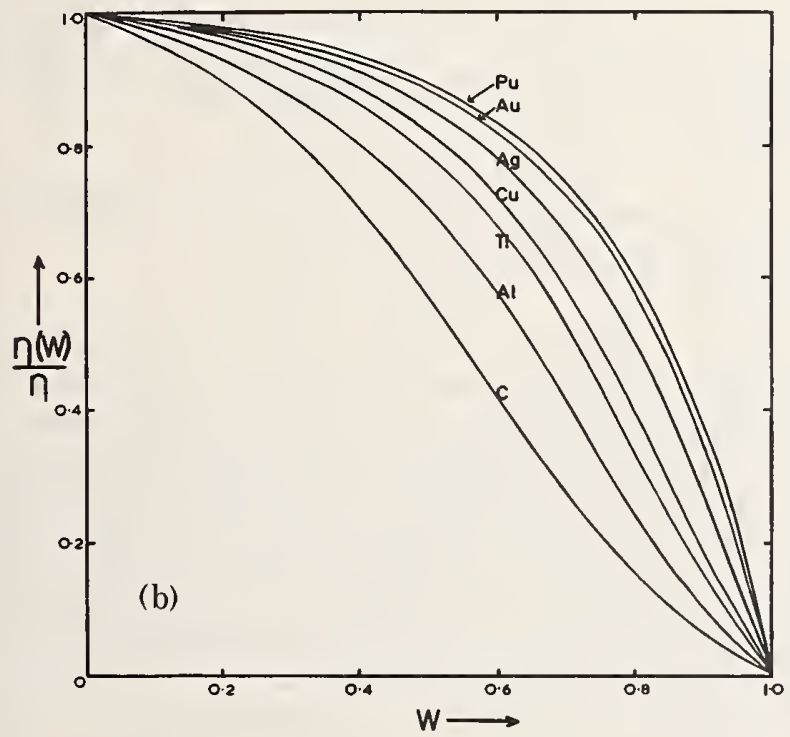

Figure 3(b). Integral form of energy distributions, with interpolated curves for aluminium, titanium, and plutonium. 


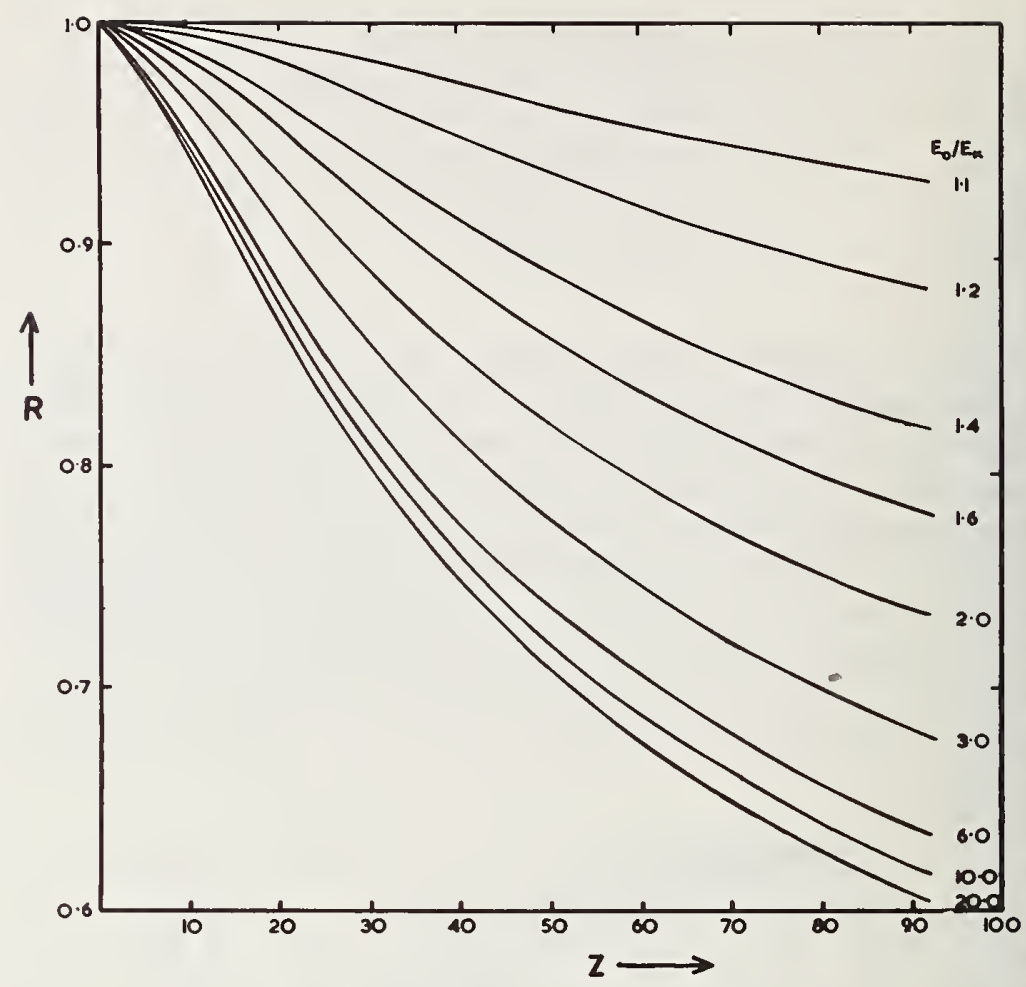

Figure 4. Fraction of ionization $\mathrm{R}$ remaining in target after loss due to backscatter, calculated from Bishop's data given in Figures 2 and 3(b).

The $\mathrm{R}$ curves in Figure 4 are fairly close to those obtained by Green [15] using a similar method with earlier backscattering data and agree well with some direct experimental measurements by Derian [16]. The curves published by Thomas [17] are, however, significantly different. The difference can be attributed firstly to the inexact method of integration used by Thomas, secondly to electron backscattering data of doubtful accuracy and thirdly to the fact that the lowest atomic number for which Thomas calculated $\mathrm{R}$ was 29 , the curves being extrapolated to $R=1$ at $Z=0$ for all $U$. Figure 4 shows an inflexion in the curves in this region, implicit in the general way the backscattered electron energy distribution varies with atomic number. A linear extrapolation is therefore inaccurate, and introduces significant errors in the area where the commonest elements occur. 
In carrying out the atomic number correction, the problem arises of how to calculate $\mathbf{R}$ for compounds. Experimental measurements of $\eta$ show that the mass concentration average

$$
\eta=\Sigma_{\mathbf{i}} \mathrm{C}_{\mathbf{i}} \eta_{\mathbf{i}}
$$

gives quite accurate results for compounds. It is therefore reasonable to assume that the same applies to $R$, so that for a compound $\mathrm{AB}$

$$
\mathrm{R}_{A B}=\Sigma_{i} \mathrm{C}_{i} \mathrm{R}_{i}
$$

Theoretical calculations by Bishop [18] and Brown [3] are in fairly good agreement with this assumption, although Bishop has suggested that it may be preferable to average the shape of the backscattered electron energy distribution independently of $\eta$. This is a question which requires further investigation; meanwhile it is reasonable to use equation (12) for practical calculations.

It is advantageous to express $R$ as a function of $1 / \mathrm{U}$ rather than $U$, which has been used previously; as noted by Bishop [18] the variation of $\mathrm{R}$ for a given $\mathrm{Z}$ is nearly linear in $1 / \mathrm{U}$, which greatly facilitates interpolation. For practical calculations, it is convenient to have $\mathbf{R}$ in tabular form. The $\mathrm{R}$ curves in Figure 4 have therefore been converted into Table 1 , from which $R$ can be obtained by linear interpolation with respect to $\mathrm{Z}$ and $1 / \mathrm{U}$, with an interpolation error of less than $0.2 \%$. This table can readily be incorporated in a computer program for microprobe corrections.

Table 1. Values of backscatter coefficient $R$ as a function of $1 / U$ and $\mathrm{Z}$.

\begin{tabular}{|cccccccccccc}
$\mathrm{Z}^{\mathrm{I} / \mathrm{U}}$ & 0.01 & 0.10 & 0.20 & 0.30 & 0.40 & 0.50 & 0.60 & 0.70 & 0.80 & 0.90 & 1.00 \\
& & & & & & & & & & & \\
0. & 1.000 & 1.000 & 1.000 & 1.000 & 1.000 & 1.000 & 1.000 & 1.000 & 1.000 & 1.000 & 1.000 \\
10. & 0.934 & 0.944 & 0.953 & 0.961 & 0.968 & 0.975 & 0.981 & 0.988 & 0.993 & 0.997 & 1.000 \\
20. & 0.856 & 0.873 & 0.888 & 0.903 & 0.917 & 0.933 & 0.948 & 0.963 & 0.977 & 0.990 & 1.000 \\
30. & 0.786 & 0.808 & 0.828 & 0.847 & 0.867 & 0.888 & 0.911 & 0.935 & 0.959 & 0.981 & 1.000 \\
40. & 0.735 & 0.760 & 0.782 & 0.804 & 0.827 & 0.851 & 0.878 & 0.907 & 0.938 & 0.970 & 1.000 \\
50. & 0.693 & 0.718 & 0.741 & 0.764 & 0.789 & 0.817 & 0.847 & 0.881 & 0.919 & 0.959 & 1.000 \\
60. & 0.662 & 0.688 & 0.713 & 0.737 & 0.764 & 0.793 & 0.825 & 0.862 & 0.904 & 0.950 & 1.000 \\
70. & 0.635 & 0.663 & 0.687 & 0.713 & 0.740 & 0.770 & 0.805 & 0.844 & 0.889 & 0.941 & 1.000 \\
80. & 0.611 & 0.639 & 0.665 & 0.691 & 0.718 & 0.750 & 0.785 & 0.826 & 0.874 & 0.932 & 1.000 \\
90. & 0.592 & 0.613 & 0.639 & 0.665 & 0.695 & 0.730 & 0.767 & 0.811 & 0.862 & 0.924 & 1.000 \\
99. & 0.578 & 0.606 & 0.634 & 0.661 & 0.691 & 0.725 & 0.763 & 0.806 & 0.858 & 0.921 & 1.000
\end{tabular}

\section{Evaluation of Stopping Power S}

From equation (8), it follows that the ratio $\mathrm{k}_{A}$ of the characteristic $x$-ray intensity from the specimen to that from the standard, neglecting, for the moment, absorption and fluorescence, is given by 


$$
k_{A}=C_{A} \cdot \frac{R_{A B}}{R_{A}} \cdot \frac{\int_{E_{K}} E_{o} Q / S_{A B} \cdot d E}{\int_{E_{K}} E_{o} Q / S_{A} \cdot d E}
$$

where suffixes $A B$ and $A$ refer respectively to the specimen and the standard. Equation (13) can be simplified with very little loss of accuracy by virtue of the fact that $\mathrm{S}_{A} / \mathrm{S}_{A B}$ varies only slowly with $\mathrm{E}$. This ratio can therefore be evaluated at an energy halfway between $\mathrm{E}_{o}$ and $\mathrm{E}_{\kappa}(17)$, and taken outside the integrals, which then cancel. Thus equation (13) may be written

$$
\mathrm{k}_{A}=\mathrm{C}_{A} \cdot\left(\mathrm{R}_{A B} / \mathrm{R}_{A}\right) \cdot\left(\overline{\mathrm{S}}_{A} / \overline{\mathrm{S}}_{A B}\right)
$$

where $\overline{\mathrm{S}}$ is the mean stopping power obtained by substituting for $\mathrm{E}$ in equation (5), a value $\overline{\mathrm{E}}$ given by

$$
\overline{\mathrm{E}}=(1 / 2)\left(\mathrm{E}_{\mathrm{o}}+\mathrm{E}_{K}\right)
$$

The atomic number correction thus reduces to two separate factors, one taking account of backscattering and the other of differences in mean stopping power between specimen and standard. It is not necessary to use the full formula for $\overline{\mathrm{S}}$ in equation (14), since the $1 / \mathrm{E}$ term cancels out. For practical correction calculations, $1 / \mathrm{E}$ may therefore be dropped from equation (5).

In order to check the error introduced by eliminating the integrals in equation (13) in favor of the $\mathrm{S}$ approximation, the relative difference between the resulting correction factors has been evaluated for a large number of extreme cases. Although it is not possible to state simply the conditions within which the approximation is valid, the following points emerge:

a. The relative error is worst when analyzing a trace of one element in another, and in general increases with difference of atomic number.

b. The relative error is worst at a $U$ value of $1.5-2$, presumably because the ionization cross-section $\mathrm{Q}$ is changing most rapidly in this region.

c. At $\mathrm{U}=2$, the relative error, defined as $[\mathrm{k} / \mathrm{C}$ (integrated)$\mathrm{k} / \mathrm{C}(\overline{\mathrm{S}})] / \mathrm{k} / \mathrm{C}$ (integrated), is negative for light elements in a heavy matrix, and positive for the reverse situation.

To illustrate the magnitude of the error, we take trace amounts of aluminium, copper and uranium in matrices of each of the other two elements, making six systems in all. For $\mathrm{Cu} \mathrm{K} \alpha$ and $\mathrm{UL} \alpha$, the error 
is evaluated in the worst excitation condition of $U=2$, but since this corresponds to only $3 \mathrm{kV}$ for $\mathrm{Al} \mathrm{K} \alpha$, a beam energy of $10 \mathrm{kV}$ is used in this case. The results are shown in Table 2. Even in these extreme conditions the error is seen to be less than $1 \%$ in all systems except for $\mathrm{Al}$ in $\mathrm{U}$, where the severe absorption correction would in any case prevent an analysis to this order of accuracy. For the $\mathrm{Al}-\mathrm{Cu}$ and $\mathrm{Cu}-\mathrm{Al}$ systems the error is very small indeed. We conclude, therefore, that in the vast majority of analyses involving elements in the atomic number range of about 13-92, the error introduced by use of the $\overline{\mathrm{S}}$ approximation is negligible. This also applies to the analysis of the heavier component of most carbides and oxides, but not to the lighter, where the absorption correction is in any case very high. .

Table 2. Error introduced in correction factor by using the $\overline{\mathrm{S}}$ approximation in a number of extreme cases.

Rel. error in

System

Excitation conditions

$\begin{aligned} \mathrm{U} & =2 \\ \mathrm{U} & =2 \\ \mathrm{U} & =2 \\ \mathrm{U} & =2 \\ \mathrm{E}_{o} & =10 \mathrm{kV} \\ \mathrm{E}_{0} & =10 \mathrm{kV}\end{aligned}$

$0.57 \%$

$0.86 \%$

$-0.81 \%$

$0.38 \%$

$2.31 \%$

$0.04 \%$

Thomas [17], having suggested the $\overline{\mathrm{S}}$ simplification described above, introduced a further assumption first put forward by Castaing, by which both parts of the atomic number correction are combined in the $\alpha$ coefficient. In this method, which is also used by Ziebold and Ogilvie [19], equation (14) becomes

$$
\mathrm{k}_{A}=\mathrm{C}_{A}\left(\alpha_{A} / \Sigma_{i} \mathrm{C}_{i} \alpha_{i}\right)
$$

where $\alpha=\mathrm{S}_{i} / \mathbf{R}_{i}$. This gives somewhat different results from separate averaging of $\mathrm{S}$ and $\mathrm{R}$ (equations 4 and 12), which we have already found to be the preferred method on theoretical grounds, and in many cases the error introduced is found to be as high as $1-4 \%$. Furthermore, in contrast with the $\overline{\mathrm{S}}$ approximation, this error is worst for concentrations in the region of $50 \%$ which are often the ones that have to be determined with greatest accuracy. It would seem, therefore, that the slight advantage of the $\alpha$ method in requiring only one averaging calculation is outweighed by the significant error introduced. 
The only remaining aspect of the calculation of the atomic number correction which requires consideration is the evaluation of $\mathbf{J}$ in the formula for $\mathbf{S}$. The available information about $\mathbf{J}$ is inadequate to supply exact numerical values, although experimental data suggest that $\mathrm{J} / \mathrm{Z}$ generally lies between 10 and $15 \mathrm{eV}$. It is therefore necessary to use values of $\mathbf{J}$ determined empirically from microprobe analysis of compounds of known composition. This procedure has the advantage that the overall effect of small errors in other aspects of the atomic number correction is automatically corrected, but is obviously dependent on the quality of the microprobe data used.

For the empirical determination of $\mathbf{J}$, we have used analyses by several different laboratories of a number of known binary compounds, collected by Poole and Thomas [13], to which have been added some analyses by the present authors totalling 48 in all. Only cases in which the absorption and fluorescence corrections are small $(<2 \%)$ have been used, to ensure that errors in these other corrections cannot influence the results. A consequence of this selection is that all the analyses used are of a heavy element in a light matrix, but this should not affect the generality of the results.

Table 3. Alloys Used in the Determination of $\mathbf{J}$

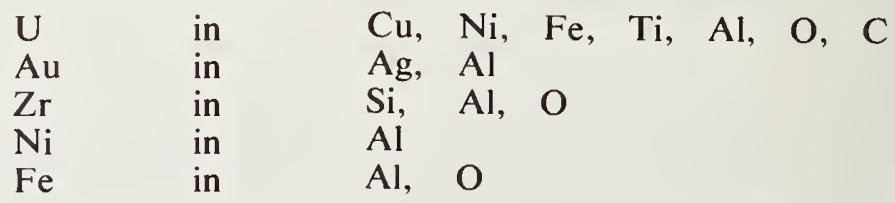

Range of concentration

Range of kilovoltage

Total number of measurements :

20 to $95 \% \mathrm{~A}$ in $\mathrm{B}$ 10 to $35 \mathrm{kV}$ 48

The elements analyzed are shown in Table 3 and give a good coverage of the periodic table from uranium to carbon. The curve of $\mathrm{J} / \mathrm{Z}$ against $\mathrm{Z}$ which gave the best overall results for these analyses is shown in Figure 5, and was obtained in the following way. The value of $\mathrm{J}$ for copper is probably the best known existing figure, and is given by $(\mathrm{J} / \mathrm{Z})_{C u}=13.0 \mathrm{eV}$. Starting with this value, a U-Cu alloy gave an initial value for uranium, setting $(\mathrm{J} / \mathrm{Z})_{U}=13.7 \mathrm{eV}$ to give the best agreement between true concentration of uranium and the value calculated from experiment. Analyses of U-Al, U-Ti, U-Fe and U$\mathrm{Ni}$ alloys then gave points from which a curve from uranium to 


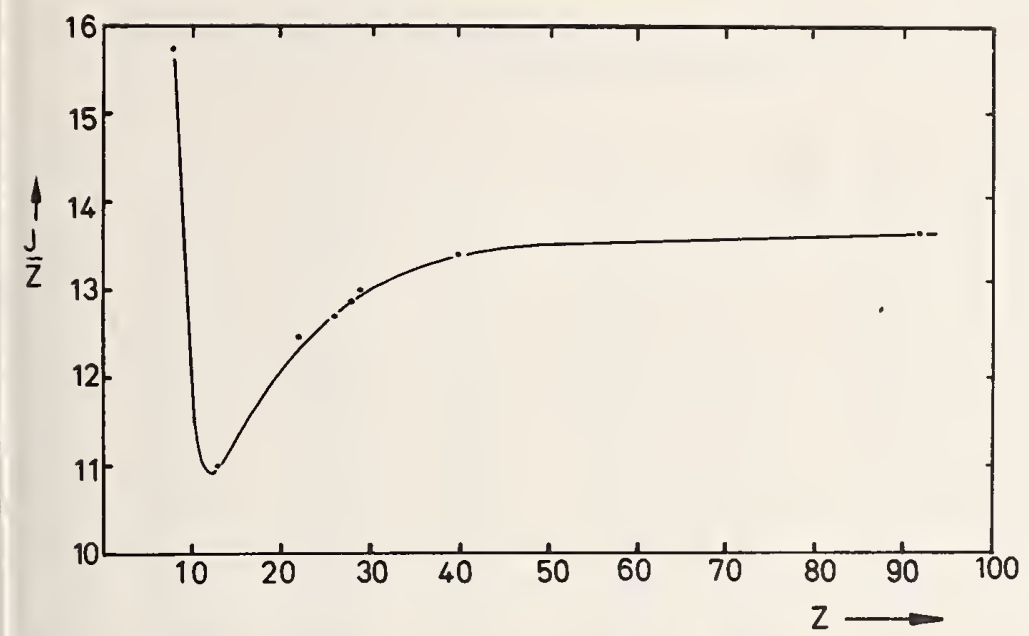

Figure 5. Mean ionization potential $\mathbf{J}$ plotted as $\mathrm{J} / \mathrm{Z}$ against $\mathrm{Z}$ determined empirically from a series of microprobe analyses of known alloys.

aluminium $(Z=13)$ could be constructed, taking into account analyses of $\mathrm{Fe}-\mathrm{Al}, \mathrm{Ni}-\mathrm{Al}, \mathrm{Zr}-\mathrm{Al}, \mathrm{Zr}-\mathrm{Si}, \mathrm{Au}-\mathrm{Ag}$ and $\mathrm{Au}-\mathrm{Al}$ alloys. $V$ alues of $\mathbf{J} / Z$ for carbon and oxygen calculated from analyses of $U$ $0, \mathrm{U}-\mathrm{C}, \mathrm{Zr}-0$ and $\mathrm{Fe}-0$ compounds gave a sharp rise in $\mathrm{J} / \mathrm{Z}$ at low atomic numbers, but apart from this rise, the variation of $J / Z$ with $Z$ is rather slow. Although some scatter was evident where $J / Z$ was determined from several analyses, this was generally less than $\pm 0.25 \mathrm{eV}$. It therefore appears to be valid to assign a unique $\mathrm{J}$ value to each element at least in the range of accelerating voltages covered (10-35 kV) and for the types of compound represented in this set of data.

No obvious physical reason can be advanced to explain this sudden rise in $\mathrm{J} / \mathrm{Z}$ at low atomic number, which has also been observed by Caldwell $[20] . \mathrm{J} / \mathrm{Z}$ calculated in this manner is influenced to a certain extent by the $R$ values used and the averaging method employed but this does not account for the rise, as need for it is found to be common to all methods. Neither is it explained by the error due to fluorescence from the continuous spectrum, which has been checked in a number of cases by the method due to Hénoc [21].

An analytical expression has been fitted to the $\mathbf{J} / \mathrm{Z}$ curve in Figure 5 and this formula has been used to calculate $\mathrm{J}$ for all $\mathrm{Z}$ between 6 and 92. These values are given in Table 4. The behavior of $J / Z$ 
Table 4. Mean ionization potential J determined empirically as a function of atomic number $Z$.

\begin{tabular}{rrrrrrrr|}
$\mathrm{Z}$ & $\mathrm{J}(\mathrm{eV})$ & $\mathrm{Z}$ & $\mathrm{J}(\mathrm{eV})$ & $\mathrm{Z}$ & $\mathrm{J}(\mathrm{eV})$ & $\mathrm{Z}$ & $\mathrm{J}(\mathrm{eV})$ \\
6 & 146 & 29 & 377 & 52 & 706 & 75 & 1017 \\
7 & 135 & 30 & 392 & 53 & 720 & 76 & 1031 \\
8 & 127 & 31 & 407 & 54 & 734 & 77 & 1044 \\
9 & 123 & 32 & 422 & 55 & 747 & 78 & 1057 \\
10 & 123 & 33 & 437 & 56 & 761 & 79 & 1071 \\
11 & 126 & 34 & 451 & 57 & 775 & 80 & 1084 \\
12 & 133 & 35 & 466 & 58 & 788 & 81 & 1097 \\
13 & 142 & 36 & 481 & 59 & 802 & 82 & 1111 \\
14 & 154 & 37 & 495 & 60 & 815 & 83 & 1124 \\
15 & 166 & 38 & 510 & 61 & 829 & 84 & 1137 \\
16 & 180 & 39 & 524 & 62 & 843 & 85 & 1151 \\
17 & 194 & 40 & 538 & 63 & 856 & 86 & 1164 \\
18 & 209 & 41 & 553 & 64 & 870 & 87 & 1177 \\
19 & 224 & 42 & 567 & 65 & 883 & 88 & 1191 \\
20 & 239 & 43 & 581 & 66 & 897 & 89 & 1204 \\
21 & 255 & 44 & 595 & 67 & 910 & 90 & 1217 \\
22 & 270 & 45 & 609 & 68 & 923 & 91 & 1231 \\
23 & 286 & 46 & 623 & 69 & 937 & 92 & 1244 \\
24 & 301 & 47 & 637 & 70 & 950 & 93 & 1257 \\
25 & 316 & 48 & 651 & 71 & 964 & 94 & $127 \mathrm{C}$ \\
26 & 332 & 49 & 665 & 72 & 977 & & \\
27 & 347 & 50 & 679 & 73 & 991 & & \\
28 & 362 & 51 & 692 & 74 & 1004 & &
\end{tabular}

below carbon $(\mathrm{Z}=6)$ is uncertain and the best procedure for lighter elements is probably to assume $\mathrm{J} / \mathrm{Z}$ is constant and equal to the value for carbon.

Using these values for $\mathbf{J}$, the relative error in the correction factor. defined as

$$
\text { Relative error }=\frac{\left(\frac{\mathrm{k}_{\mathrm{A}}}{\mathrm{C}_{\mathrm{A}}}\right) \exp ^{-}\left(\frac{\mathrm{k}_{\mathrm{A}}}{\mathrm{C}_{\mathrm{A}}}\right) \text { theory }}{\left(\frac{\mathrm{k}_{\mathrm{A}}}{\mathrm{C}_{\mathrm{A}}}\right) \text { theory }}
$$


has been calculated for the 48 binary compounds. The results are presented in the form of a histogram in Figure 6(a). Of the 48 alloys, 24 show a relative error of better than $\pm 0.5 \%$, and all but 2 are within $\pm 2.5 \%$. In Figure 6 (b) the results are recalculated, setting $\mathrm{J} / \mathrm{Z}=11.5 \mathrm{eV}$, as has been widely used in the past; the increased errors found in comparison with Figure 6(a) are readily apparent, only 6 analyses giving an error less than $\pm 0.5 \%$. Finally in Figure 6(c) the uncorrected $\mathrm{k}_{A}$ values are plotted in a similar way, to emphasize the need for making the atomic number correction. Thus errors extending up to $22 \%$ are reduced by nearly an order of magnitude if the present procedure is followed. Taking into account the undoubted existence of errors in the analyses, this is as good as can reasonably be expected, though as more and more accurate results become available, it may be necessary to modify the $\mathrm{J} / \mathrm{Z}$ function still further.

\section{Accuracy of the Atomic Number Correction in Practice}

It is important to know the accuracy of quantitative microprobe analysis. Reproducibility is governed by instrumental factors and $\mathrm{x}-$ ray counting statistics, whereas absolute accuracy depends on the accuracy of the correction theory. Considering first the backscattering factor in the atomic number correction, we see from equation (10), that a given relative error in $\eta$ gives rise to an equal relative error in $1-\mathrm{R}$. Therefore there will be very little error in $\mathrm{R}$ for elements of low atomic number for which $\mathrm{R}$ is fairly close to unity. We estimate that $R$ for elements with $Z<30$, which includes most of the common elements, is accurate to $\pm 1 \%$.

The position is less satisfactory for heavy elements, especially for $Z>50$ where both $\eta$ and the energy distribution of backscattered electrons are appreciably dependent on $\mathrm{E}_{o}$. For instance, Bishop [10] found that $\eta$ for uranium decreases from 0.534 at $30 \mathrm{keV}$ to 0.495 at $5 \mathrm{keV}$, corresponding to a $5 \%$ increase in $\mathrm{R}$. This variation in $\mathbf{R}$ is further accentuated by the change in shape of the energy distribution with $\mathrm{E}_{\boldsymbol{o}}$. The values of $\mathrm{R}$ in Table 1 were calculated for $20 \mathrm{keV}$, so appreciable errors may arise if different accelerating voltages are used, particularly if they are below about $10 \mathrm{kV}$, when the tabulated values of $\mathbf{R}$ may be several percent too low. It is desirable that in due course values of $\mathbf{R}$ for heavy elements, which allow for the dependence on $\mathrm{E}_{o}$, should be calculated, and this is an area which would benefit from further experimental investigations of backscattering. 


\section{FITTED J VALUES}
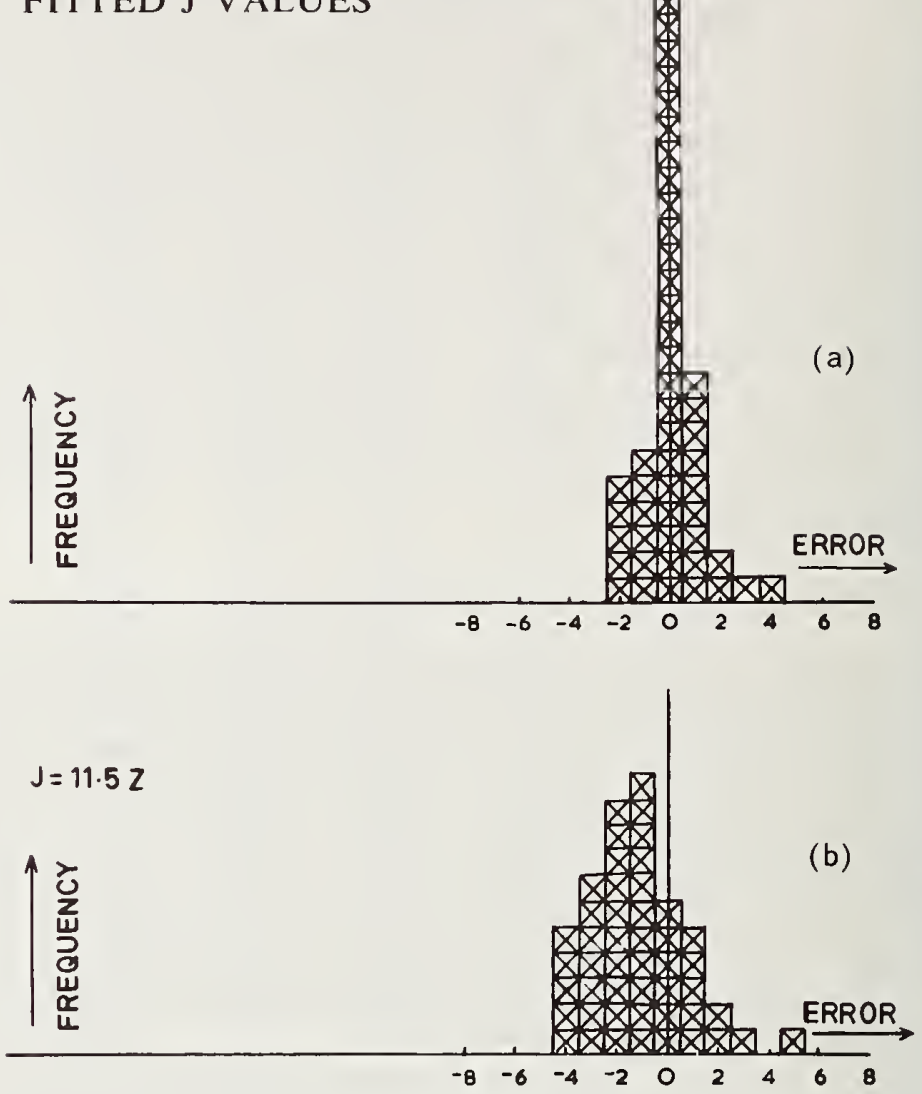

UNCORRECTED RESULTS

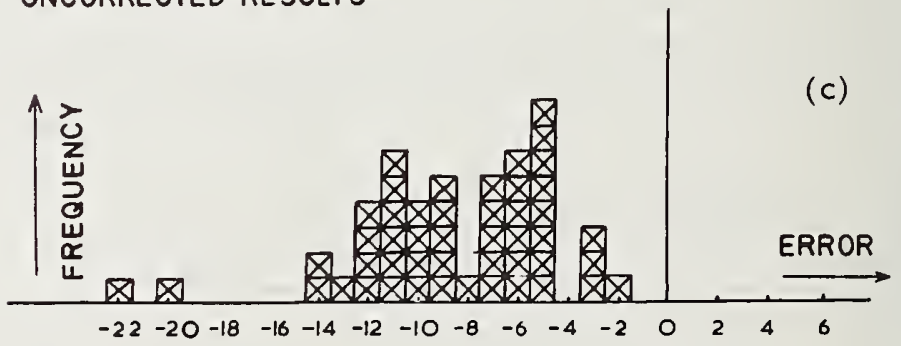

Figure 6. Histograms showing relative error between measured and calculated intensity ratios for 48 known alloys (a) corrected using empirical $\mathrm{J} / \mathrm{Z}$ function of Figure 5 (b) setting $\mathrm{J} / \mathrm{Z}=11.5 \mathrm{eV}$ (c) with no atomic number correction at all. 
The mass concentration averaging of $\mathrm{R}$ for compounds, expressed in equation (12), may not be precisely correct, but theoretical calculations (18) of $\mathrm{R}$ for compounds suggest that the error is not much more than $1 \%$ in bad cases, and it will often be much less in practice. Experimental investigations of electron backscattering from compounds would be most helpful in deciding whether a more refined method of averaging would be more accurate. Furthermore, if this and other aspects of backscattering could be studied with nonnormal angles of incidence of the electron beam, it would make it possible to calculate values of $\mathbf{R}$ suitable for those instruments in which the probe is inclined to the perpendicular from the specimen surface. Present indications are, however, that the effect of inclining the electron beam on the atomic number correction is small.

According to equation (5), the stopping power becomes negative when $1.166 \mathrm{E}<\mathrm{J}$. This is entirely due to the mathematical form of the equation, and is obviously not physically correct, but only presents a problem for very light elements beyond the range of this discussion. A part from this breakdown, there is a progressive failure of the Bethe formula as $\mathrm{E}$ approaches $\mathrm{J}$, due to $\mathrm{J}$ being constant, while in fact the effective value of $\mathbf{J}$ decreases with decreasing $\mathbf{E}$. Livingston and Bethe [22] have shown how the formula may be adapted to give the contributions to $\mathrm{S}$ of the $\mathrm{K}$ electrons and of all the other electron shells separately. In a heavy element the $\mathrm{K}$ ionization potential is considerably higher than the highest accelerating voltage used in microprobe analysis, so that the contribution of the $\mathrm{K}$ electrons to $\mathrm{S}$ can be neglected. For $\mathrm{E}=15$ $\mathrm{keV}$, the Livingston-Bethe formula indicates that the true stopping power of uranium is $1.4 \%$ higher than that given by equation (5), while if the estimated L-shell effect is taken into account, the difference becomes about 3\%. A similar calculation for copper suggests a difference of about $2 \%$ in the same direction. $\mathrm{K}$-shell effects in light elements, for which $\overline{\mathrm{E}} \gg \mathrm{E}_{K}$, are very small. Therefore if the true mean ionization potential wère used in equation (5) for the atomic number correction, $\mathrm{S}$ would be underestimated by an amount which increases with $\mathbf{Z}$. However, the use of empirical values of $\mathbf{J}$ automatically tends to take account of K- and L-shell effects, and will reduce errors due to this cause.

The possible effect of the chemical state of the atoms in the specimen has been neglected so far. Brandt [23] has calculated $\mathbf{J}$ for free and bound atoms of various elements. Appreciable differences were found; for instance a value of $109 \mathrm{eV}$ was obtained for aluminium as free atoms, compared with $149 \mathrm{eV}$ in the metallic 
state. The corresponding figures for iron were $211 \mathrm{eV}$ and $241 \mathrm{eV}$. No doubt variations in $\mathbf{J}$ for a given element in different solid specimens are much less than this, but if Brandt's calculations are correct, chemical differences may affect stopping power by up to $2 \%$. This makes it desirable for the analyzed element to be in a similar chemical state in both standard and specimen, which also minimizes chemical wavelength shifts.

In the error histogram in Figure 6(a), $81 \%$ of the analyses in which the absorption correction is small lie within $\pm 1.5 \%$ of the zero error line, which gives some indication of the general accuracy of the atomic number correction. However, the specimens vary widely in the atomic numbers of the constituent elements and the size of the correction. In the range $12<\mathrm{Z}<30,10 \mathrm{keV}<\mathrm{E}_{\theta}<30 \mathrm{keV}$, within which a large proportion of practical cases lie, the atomic number correction factor is generally between 0.75 and 1.35 , and an accuracy of $5 \%$ to $10 \%$ of the difference from unity is a realistic estimate. Thus an error of $3 \%$ of the concentration is possible in the worst cases. It is obviously desirable to use as a standard something which approximates, even if only roughly, to the composition of the specimen. If the correction factor is kept within the limits 0.90 to 1.10 the error in the corrected concentration should be less than $\pm 1 \%$.

For heavy elements accuracy decreases, mainly due to increasing uncertainty in $\mathrm{R}$. The error in the correction factor is least for accelerating voltages around $20 \mathrm{kV}$, but may rise to $\pm 20 \%$ of the difference from unity for the heaviest elements in combination with light elements, increasing still further as the voltage is lowered. It is preferable to analyze such elements at not less than $20 \mathrm{kV}$, though this often results in a large absorption correction, so that the overall accuracy may in any case be limited.

With regard to specimens containing elements less than 12 in atomic number, the above procedure may be used for the analysis of the heavy component, but not of the light. Not only does the $\overline{\mathrm{S}}$ approximation fail, but backscattering data are unreliable for the low voltages used for excitation of the light elements. In view of the large absorption correction to be expected in this region, some other procedure, such as the surface layer method reported by Duncumb and Melford [24], would be more appropriate.

So far the absorption and fluorescence corrections have been ignored. It is outside the scope of this paper to describe how to calculate them, but we will briefly consider their combination with the atomic number correction. Some confusion has arisen in the past 
in attempting to make the atomic number correction an implicit part of the absorption correction. In principle this is possible as discussed by Duncumb and Shields [25] but in practice it is not possible to represent the distribution of ionization with depth to sufficient accuracy, to give a good measure of the total intensity generated as a function of atomic number. For this reason, we recommend that the atomic number correction, which, as noted earlier, applies to the generation of $\mathrm{x}$-rays, is treated as a separate factor. The absorption correction is then merely a ratio of $f(\chi)$ for the specimen to that of the standard, where $f(\chi)$ is the fractional transmission of the target for $\mathrm{x}$-rays which emerge in the direction of the spectrometer. It is true that $f(\chi)$ is dependent on atomic number, but this is not to be confused with the "atomic number correction". A method of calculating $f(\chi)$, based on that of Philibert, has been put forward by Duncumb and Shields [26]. The third part of the correction, that for fluorescence, is simply the relative enhancement of the measured intensity due to fluorescence, and may be calculated by the method described by Reed [27].

Referring back to equation (2), we are thus able to calculate the overall correction factor for any system of known composition. In the practical case where the composition is not known, it is necessary to start with an assumed composition which is successively improved by an iterative procedure until the measured intensity ratios are consistent with the calculated correction factors. This is carried out most easily with the aid of a computer, though this is by no means essential. More important is the need to develop a sound correction theory and establish its range of validity.

\section{Conclusion}

A practical method has been put forward for making the atomic number correction in the analysis of elements of atomic number 12 and upwards. This is based upon Bishop's experimental data for calculating $R$ values to allow for electron backscatter, and uses an empirical relation between $\mathrm{J} / \mathrm{Z}$ and $\mathrm{Z}$ for calculating stopping power. Although more accurate analyses of known alloys may lead to a modification of the $\mathrm{J} / \mathrm{Z}$ function, the measurements used in the present study were selected from those in which the atomic number correction was dominant, and establish the general form of the function with some certainty. The theory has been simplified as far as its fundamental limitations will permit, and is straightforward to apply with or without a computer. 


\section{Acknowledgments}

The authors gratefully acknowledge the assistance of Miss C. da Casa and Mrs. P. K. Shields Mason in the work, and are indebted to the Chairman of Tube Investments for permission to publish this paper.

\section{References}

[1] Castaing, R., Thesis, University of Paris (1951).

[2] Archard, G. D., J. Appl. Phys. 32, 1505 (1961).

[3] Brown, D. B., Thesis, M.I.T. (1965).

[4] Green, M., Proc. Phys. Soc., 82, 204 (1963).

[5] Bishop, H. E., Proc. Phys. Soc., 85, 855 (1965).

[6] Bethe, H. A., Ann. Phys. Lpz., 5, 325 (1930).

[7] Bethe, H. A. and Ashkin, J., "Experimental Nuclear Physics" Ed. Segre, John Wiley and Sons, New York, (1953).

[8] Bloch, F., Zeit. f. Phys., 81, 363 (1933).

[9] Wilson, R. R., Phys. Rev., 60, 749 (1941).

[10] Bishop, H. E., "Optique des Rayons X et Microanalyse", Hermann, Paris, (1967).

[11] Weinryb, E. and Philibert, J., Comptes Rendus, 258, 4535 (1964).

[12] Webster, D. L., Clark, H. and Hansen, W. W., Phys. Rev., 37, 115 (1931).

[13] Thomas, P. M., A.E.R.E. Rept. No. 4593, Harwell, (1964).

[14] Tomlin, S. G., Austral. J. Phys., 17, 452 (1964).

[15] Green, M., Thesis, Cambridge University, (1962).

[16] Derian, J. C., CEA Rept. No. R3052 (1966).

[17] Thomas, P. M., Brit. J. Appl. Phys., 14, 397 (1963).

[18] Bishop, H.E., Thesis, Cambridge University, (1965).

[19] Ziebold, T. O. and Ogilvie, R. E., Anal. Chem., 35, 621 (1964).

[20] Caldwell, D. O., Phys. Rev., 100, 291 (1955).

[21] Hénoc, J., C.N.E.T. Rept. No. 655 (1962).

[22] Livingston, H. S. and Bethe, H. A., Rev. Mod. Phys., 9, 245 (1937).

[23] Brandt, W., Phys. Rev., 104, 691 (1956).

[24] Duncumb, P. and Melford, D. A., Paper given at 1 st National Symposium on Electron Probe Microanalysis, Maryland, 1966.

[25] Duncumb, P. and Shields, P. K., Brit. J. Appl. Phys., 14, 617 (1963).

[26] Duncumb, P. and Shields, P. K., "The Electron Microprobe", John Wiley and Sons, New York (1966) p. 284.

[27] Reed, S. J. B., Brit. J. Appl. Phys., 16,913 (1965). 


\title{
SCATTERING OF ELECTRONS IN METALLIC TARGETS
}

\author{
GUNJI SHINODA, KENJI MURATA, \\ and RYUICHI SHIMIZU
}

\begin{abstract}
Department of Applied Physics, Osaka University, Higashinoda, Miyakojima, Osaka, Japan
\end{abstract}

\section{Summary}

Monte Carlo calculations were performed to clarify the behavior of electrons and to obtain depth distribution. functions of characteristic $\mathrm{x}$-rays in copper and aluminum targets using Lewis' multiple scattering theory and selecting the best fit values for the screening factor. The results concerning backscattering and penetration phenomena were obtained as a natural consequence of the computation process. Agreement with experimental results, both in the backscattering coefficient and the depth distribution function, is sufficient for application to other materials and problems.

The shape of the diffused $\mathrm{x}$-ray source was also determined, and a comparison was made of the resolving power of $\mathrm{X}$-ray and specimen current methods.

\section{Introduction}

It is quite certain that an appropriate approach to clarify the behavior of electrons in the specimen is to obtain an analytically rigorous solution of the transport equation. However, to apply this to the problem of electron behavior, we must consider the effect of backscattering; and it becomes very difficult to obtain such an analytically rigorous solution. On the other hand, Monte Carlo calculations would provide valuable information on the behavior of electrons in the specimen target. Therefore, at present, Monte Carlo calculation is the most appropriate approach to investigate the behavior of electrons in the target material, although the results of the calculation should be checked with the experimental results.

We have previously published the results of the determination of the depth distribution function of $\mathrm{x}$-rays in copper using three different methods, i.e. (1) tracer method [1], (2) Monte Carlo 
calculation [2], and (3) analysis of angular distribution of characteristic x-rays [3]. The agreement among the results obtained was quite good. Therefore, we propose that our Monte Carlo calculation should also be applied to many problems other than distribution functions.

Before performing this calculation, an appropriate simplified model should be chosen to describe the behavior of electrons. The Archard diffusion model [4] is very simple and enables us to understand various phenomena concerning electron penetration.

\section{Discussion}

A. THE ARCHARD DIFFUSION MODEL

Bethe et al. [5] defined the depth of complete diffusion as that in which $\langle\cos \theta\rangle_{a v}$, an expression of the mean deviation of electrons from the initial direction becomes

$$
\langle\cos \theta\rangle_{a v}=(1 / \mathrm{e})
$$

Archard further simplified this model, as shown in Figure 1.

\section{INCIDENT ELECTRONS}

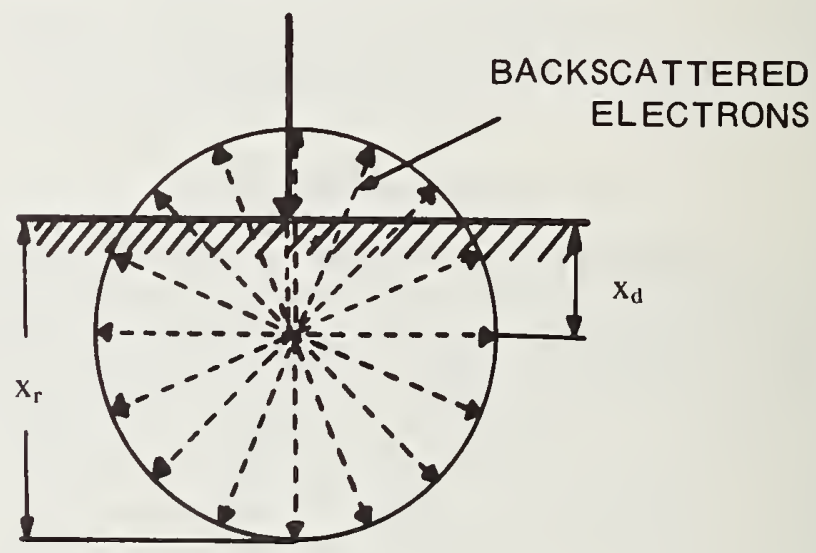

Figure 1. Archard diffusion model. 
Electrons are assumed to penetrate into the specimen to a certain depth keeping their original travel direction, and afterwards they become completely diffused; their paths are indicated by the arrows shown in Figure 1. The depth of penetration is such that the energy has changed from $\mathrm{E}_{o}$ to $\mathrm{E}_{d}$ as defined by

$$
\int_{\mathrm{E}_{d}}^{\mathrm{E}_{o}} \frac{\mathrm{dE}}{\lambda|\mathrm{dE} / \mathrm{ds}|}=\frac{1}{2}
$$

where $s$ is the distance traveled and $\lambda$ is the mean free transport path. From this formula, Archard obtained the depth of complete diffusion $\mathrm{x}_{d}$ as

$$
\mathrm{x}_{d}=\frac{40}{7 \mathrm{Z}} \mathrm{x}_{R}
$$

where $\mathrm{Z}$ is the atomic number and $\mathrm{x}_{k}$ is the range. Since the backscattered electrons are included in a cone with half apex angle $\theta$, the backscattering coefficient $\eta$ is given by the following equation

$$
\eta=\frac{1}{2}\left[1-\frac{\mathrm{x}_{d}}{\mathrm{x}_{R}-\mathrm{x}_{d}}\right]
$$

The dependence of $\eta$ upon $Z$ can be obtain using Eq. (3), and it agrees with the experimental results fairly well. These equations are deduced from the small angle multiple scattering hypothesis of Bethe et al. [5]. However, in the actual case, large-angle single scatterings are also important, although they are not included in the above treatment. As will be discussed later, the distribution function obtained from this model does not fit well the experimentally obtained values. Therefore, a better model should be tried in a Monte Carlo calculation which should contain a sufficiently high number of possible processes.

\section{B. MONTE CARLO CALCULATION}

When an electron of energy $E$ strikes a target normal to the surface at point $\mathbf{P}_{o}$ (Fig. 2), the electron suffers a large number of collisions with atoms and dissipates its energy through inelastic collisions. Most electrons lose their energy in the target and are detected as specimen current; some leave the surface and are called backscattered electrons. In the Monte Carlo calculation, it is 


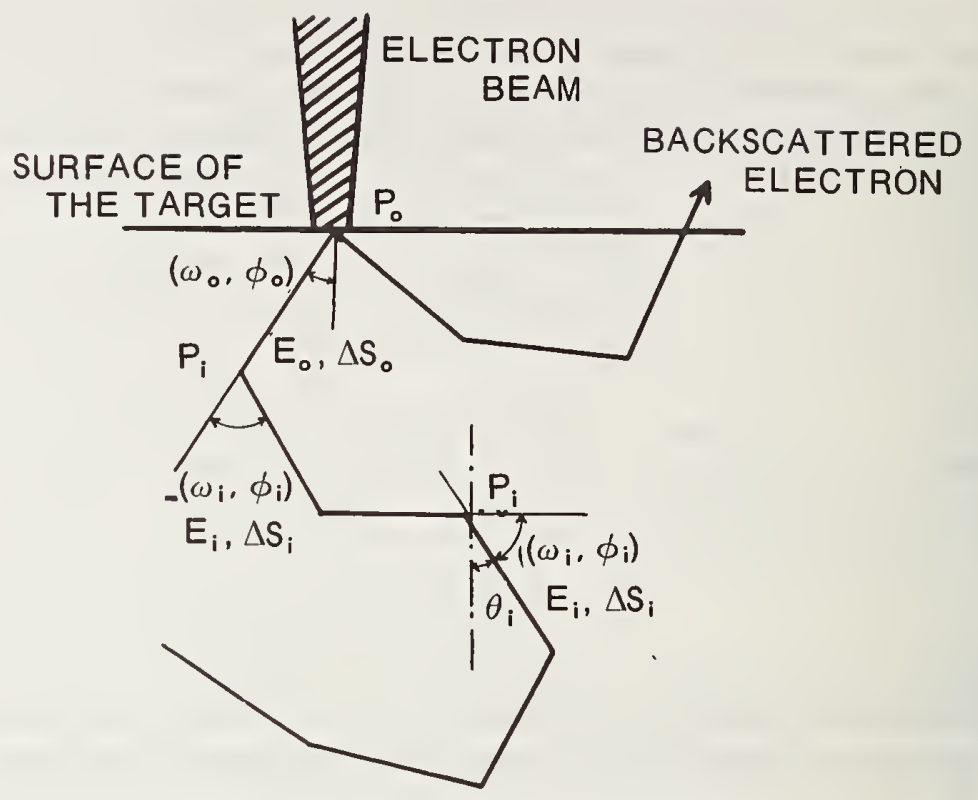

Figure 2. Simplified model of electron trajectory.

assumed that an electron travels a small distance $\Delta S_{i}$ in a straight line with constant energy $\mathbb{E}_{i}$ after having been scattered at the point $\mathbf{P}_{i}$ with a scattering angle $\left(\omega_{i}, \phi_{i}\right)$. Thus, if we take a simplified model of the electron trajectory as shown in Figure 2, the behavior of the electron in the target can be determined by the angular distribution of scattered electrons, the energy loss, and the step length. The outline of the calculation is as follows.

\section{Angular Distribution}

It would be plausible to assume that the probability of deviation about the azimuithal angle $\phi_{i}$ is uniform. However, for the zenithal angle $\omega_{i}$, the solution of the transport equation by Lewis [6] for an infinite medium has been utilized to describe the angular distribution of electron scattering $f(\omega)$ which is given for a certain path of an electron $\mathrm{S}$ as 


$$
f(\omega)=\frac{1}{4 \pi} \sum_{l=0}^{\infty}(2 l+1) \mathrm{P}_{l}(\cos \omega) \exp \left(-\int_{0}^{\mathrm{S}} \kappa_{l} \mathrm{dS}\right)
$$

where

$$
\begin{aligned}
\kappa_{l} & =2 \pi \mathrm{N} \int_{0}^{\pi} \sigma(\omega)\left[1-\mathrm{P}_{\imath}(\cos \omega)\right] \sin \omega \mathrm{d} \omega \\
& =\mathrm{A}\left\{\frac{1}{2 \beta(1+\beta)}-\frac{1}{2}(l+1) \int_{0}^{1} \frac{\lambda^{l}(1-\lambda)^{l} \mathrm{~d} \lambda}{(\lambda-\beta)^{l+2}}\right\}
\end{aligned}
$$

and $P_{l}(\cos \omega)$ is the Legendre polynomial, $\beta$ is the screening parameter, $\sigma(\omega)$ is the single scattering cross section, and $\mathrm{A}=2 \pi \mathrm{NZ}^{2} \mathrm{e}^{4} / \mathrm{p}^{2} \mathrm{v}^{2}$.

In the numerical calculation, the following approximation has been used:

$$
\int_{0}^{\Delta S} \kappa_{\imath} d S \doteq \kappa_{\imath} \cdot \Delta S
$$

Then Eq. (5) becomes the same as that of Goudsmit and Saunderson [7] for a thin layer or small $\Delta S$.

The screened Rutherford type [8] expression has been used for the single scattering cross section contained in the term $\kappa_{l}$ in Eq. (6); that is

$$
\sigma(\omega)=Z^{2} \mathrm{e}^{4} / \mathrm{p}^{2} \mathrm{v}^{2}(1-\cos \omega+2 \beta)^{2}
$$

Here the factor for inelastic collisions has been neglected since the probability that electrons are influenced by inelastic collision with large angle scattering is small enough compared to that of elastic collision if the atomic number is not very low. In the case of light elements, this effect is not negligible, and $Z^{2}$ in Eq. (7) should be replaced by $Z(Z+1)$ (Kulchetsky and Latyshev [9]).

The above angular distribution is accurate enough to describe real scattering phenomena of electrons in a given thin layer. However, some considerations on the accuracy of the equation should be made 
by comparison with experimental results of electron scattering in thin films. Then an optimum value of the screening angle may be found through comparison, since some discrepancies in the values of $\beta$ are reported (for example at $20 \mathrm{kV}$, as seen in Table 1).

Table 1. Values of screening parameter $\beta$ for copper.

\begin{aligned}$\beta \times 10^{3} & \multicolumn{1}{c}{$ Author } \\ 6.59 & Moliere [10] \\ 2.52 & Nigam et al. [11] \\ 2.01 & Wentzel [8] \\ 1.35 & Cosslett and Thomas [12] \end{aligned}

For each value of $\beta$ in Table 1 , the angular distribution $\mathbf{f}(\omega)$ was calculated using Eq. (5). The calculated angular distributions were compared with experimental data for a copper film, obtained by Cosslett and Thomas [12], and we have taken the value by Nigam et al. [11], as the best fitting value for copper at $20 \mathrm{kV}$. Before the actual calculation, the following considerations were made.

Since Eq. (5) is obtained from an assumption of multiple scattering, too small a value of $\Delta S$ is not desirable. However, experiments are usually made on thin films, and $f(\theta)$ is obtained for values of thickness $\Delta t$. If $\Delta t$ is sufficiently small we can take $\Delta t$ instead of $\Delta \mathrm{S}$. Therefore, the value of $\Delta \mathrm{t}$ that corresponds to the beginning of multiple scattering is taken. As in multiple scattering, if $f(\theta)$ is expressed by a Gaussian, then the $\log f(\theta)-\theta^{2}$ curve will be a straight line. After several trials, we have reached the conclusion that multiple scattering should occur between $\Delta \mathrm{t}=0.0901 \mu \mathrm{m}$ and $0.1403 \mu \mathrm{m}$. However, it will be very close to the former.

If $f(\theta)$ is expressed by a Gaussian, an estimate of the value of $\beta$ may be obtained from the value of $\mathrm{f}(0)$. Thus, for $\Delta \mathrm{t}=0.0901 \mu \mathrm{m}$, $\beta=2.50 \times 10^{-3}$, and for $\Delta \mathrm{t}=0.1403 \mu \mathrm{m}, \beta=3.66 \times 10^{-3}$. Among the values shown in Table 1 , the value $2.52 \times 10^{-3}$ (Nigam et al. [11]) should be the best fit for copper at $20 \mathrm{kV}$. Then

$$
\beta=\frac{1}{4}\left(1.12 \frac{\hbar \lambda}{p}\right)^{2}, \quad \lambda=Z^{1 / 3} /\left(0.885 a_{o}\right)
$$


where $\hbar$ is the reduced Planck constant and $\mathrm{a}_{0}$ is the Bohr hydrogen radius.

In Figure 3, a comparison is made with the experimental data of Cosslett and Thomas [12] in which $f(\omega)$ is normalized $f(0)$ and values of $f(0)$ are shown as $f_{e}(0)$ and $f_{c}(0)$, for experiment and calculation, respectively.

The same consideration has been made for aluminum. The Cosslett and Thomas result for $20 \mathrm{kV}$ shows that multiple scattering is found for $\rho \Delta \mathrm{t}=150 \mu \mathrm{g} / \mathrm{cm}^{2}$. From Eq. (5) $\beta$ has been calculated and $\mathrm{f}(\theta)$ was also obtained. The thick curve of Figure 4 shows the theoretically obtained result. Agreement between theory and experiment is satisfactory.

\section{Energy Loss}

The energy loss of electrons in the target was calculated using the relation given by Bethe [13]

$$
-(\mathrm{dE} / \mathrm{dS})=\left(2 \pi \mathrm{e}^{4} \mathrm{NZ} / \mathrm{E}\right) \ln (2 \mathrm{E} / \mathrm{J})
$$

where $J$ is the mean excitation potential, Wilson's value [14] of $\mathrm{J}=11.5 \mathrm{eV}$ being taken. Then the energy of the electron at the $\mathrm{i}$-th step, $\mathrm{E}_{i}$, is given by the following relation

$$
\begin{aligned}
\mathrm{E}_{i}= & \mathrm{E}_{i-1}+(\mathrm{dE} / \mathrm{dS}) \cdot \Delta \mathrm{S}_{i-1} \\
& \text { 3. Step Length }
\end{aligned}
$$

The selection of the step length is constrained by two opposing factors. To estimate the real phenomenon of electron scattering more accurately, shorter step lengths are preferable in the Monte Carlo method. But on the other hand, an appropriate step length is required to satisfy the treatment of multiple scattering and to obtain mathematically a stable convergence of Eq. (5). In the present calculation, this problem was resolved as follows.

The convergence of Eq. (5) depends on the exponential term in it, and the $\kappa_{l}$-values converge to a certain value $\kappa_{l_{\max }}$ as the value of $l$ becomes large. Then as can be easily derived from Eq. (6), the relation for $\kappa_{l \max }$ is

$$
\kappa_{l \max } \Delta \mathrm{S}=\mathrm{A} \Delta \mathrm{S} / 2 \beta(1+\beta)
$$

where $A$ varies as $1 / E^{2}$ and $\beta$ varies as $1 / E$. Since the condition $1 \gg \beta$ is satisfied if the step length is given for electrons of certain entrgy $\mathrm{E}_{i}$ by

$$
\Delta \mathrm{S}_{i}=\left(\mathrm{E}_{i} / \mathrm{E}_{o}\right) \cdot \Delta \mathrm{S}_{o}
$$




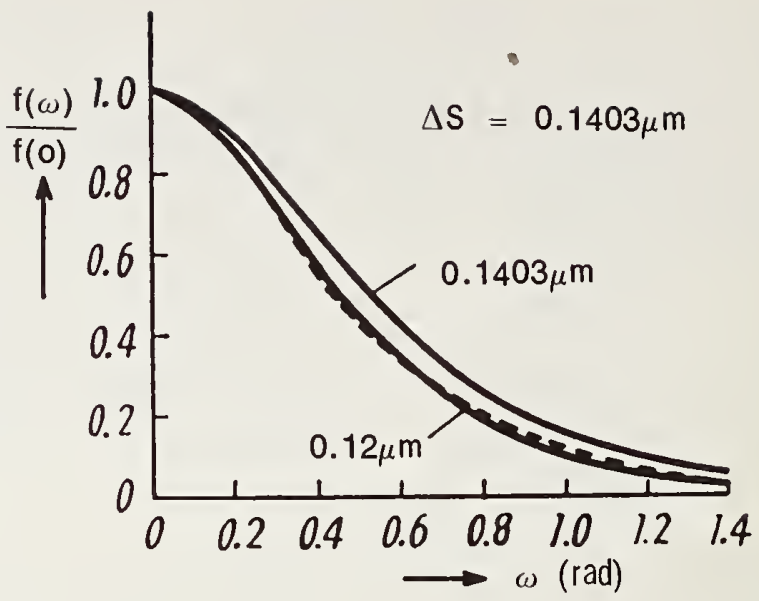

$$
\begin{aligned}
& f_{c}(0)=0.628(\Delta S=0.1403 \mu \mathrm{m}) \\
& f_{c}(0)=0.808(\Delta S=0.12 \mu \mathrm{m}) \\
& f_{e}(0)=0.786
\end{aligned}
$$

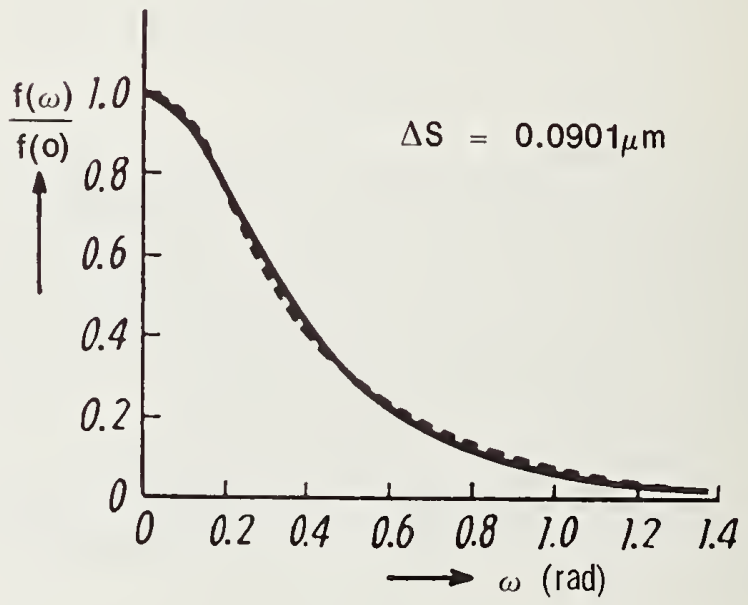

$$
\begin{aligned}
& f_{c}(0)=1.25 \\
& f_{e}(0)=1.24
\end{aligned}
$$

Figure 3. Comparison of calculated angular distribution with experiment for copper at $20 \mathrm{kV}$.

\footnotetext{
Theoretical, by Eq. (5) : $\beta=2.52 \times 10^{-3}$.

- _ - Experimental, (Cosslett and Thomas).
} 


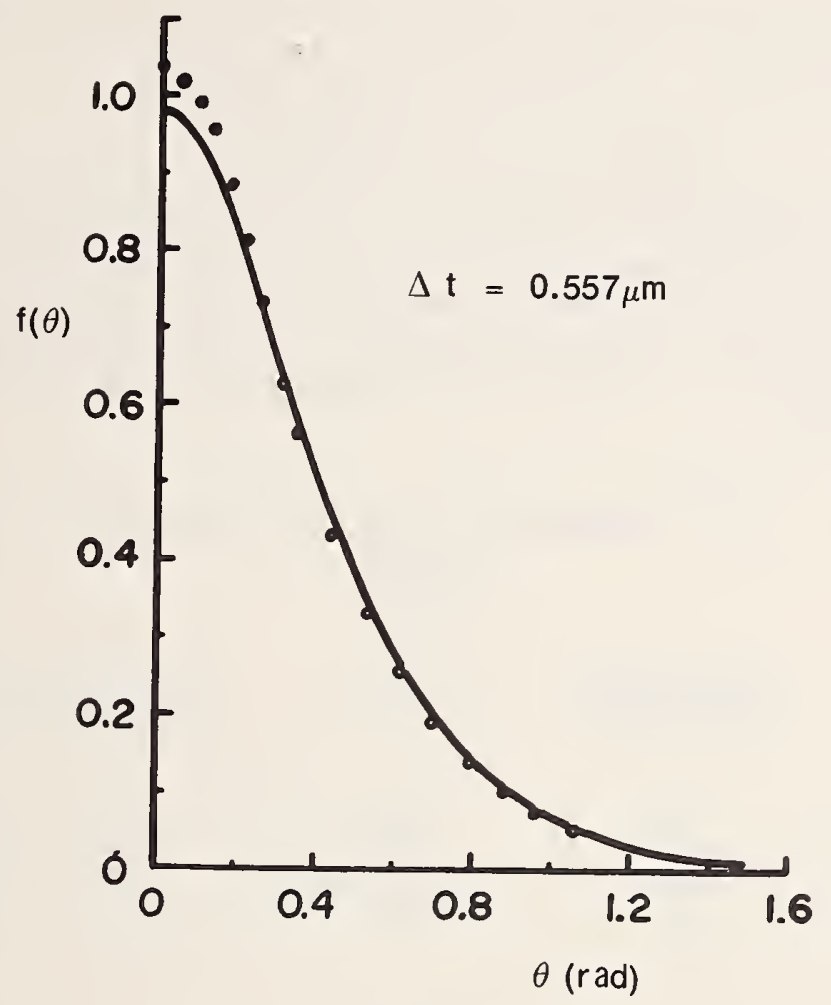

Figure 4. Comparison of calculated angular distribution with experiment for aluminum at $20 \mathrm{kV}$.

Theoretical, by Eq. (5).

- Experimental values of R. N. Thomas

(Thesis, Cambridge University, 1961).

the relation

$$
\kappa_{\text {lmax }} \Delta \mathrm{S}_{i=\mathrm{k}}\left(\Delta \mathrm{S}_{o} / \mathrm{E}_{o}\right)
$$

is independent of $E_{i}$. Thus, the above relation gives the lower limit of step length for convergence of Eq. (5). Accordingly, the calculation of electron scattering can be carried on with nearly equal precision at each step; and moreover, the step length can be taken smaller at 
the region where $\mathrm{dE} / \mathrm{dS}$ shows a greater gradient and vice versa. The step length in the present calculation is, for example, $1.8 \times 10^{-5} \mathrm{~cm}$ for copper at $30 \mathrm{kV}$, and $8.0 \times 10^{-5} \mathrm{~cm}$ for aluminum at $35 \mathrm{kV}$ )

\section{Calculation Procedure}

The calculation was carried out using an IBM $7074^{1}$ computer, for accelerating voltages of $25.1,30,34.7$ and $40 \mathrm{kV}$ for copper, and $19.8,25.1,29.3$ and $35 \mathrm{kV}$ for aluminum. The numbers of steps and trajectories are shown in Table 2. The statistical errors of calculation for the values of the backscatter ratio are about \pm 0.01 at all voltages.

Table 2. Numbers of steps and trajectories at various accelerating voltages.

Copper

Accelerating voltage, $\mathrm{kV}$

25.1

30.0

34.7

40.0

$\begin{array}{cc} & \text { Aluminu } \\ \begin{array}{c}\text { Accelerating } \\ \text { voltage, } \mathrm{kV}\end{array} & \begin{array}{c}\text { Numbe } \\ \text { of steps }\end{array} \\ 19.8 & 18 \\ 25.1 & 22 \\ 29.3 & 25 \\ 35.0 & 29\end{array}$

\section{Aluminum}

Number
Number of trajectories of steps

20

25

30

35

2000

4000

2000

2000

Number of

trajectories

2000

2000

2000

2000

${ }^{1}$ The authors appreciate the kindness of Nippon Kokan Co. Ltd. 
C. RESUlTS OF THE CALCULATIONS

\section{Backscattering of Electrons}

Examples of energy distributions of backscattered electrons are shown in Figure 5, and agreement with the experimental data of Kulenkampff and Spyra [15] is satisfactory. These authors have shown that the energy distribution is independent of the accelerating voltage between 20 and $40 \mathrm{kV}$. This point was recently confirmed by Burkhalter [16]. Our Monte Carlo calculation has given nearly the same result, as shown in Table 3.

Table 3. Comparison of backscatter coefficients.

$\eta_{M}$ : calculated $\eta_{E}$ : experimental

Copper

Accelerating voltage, $\mathrm{kV}$

$\eta_{M}$

25.1

30.0

34.7

40.0
$0.347 \pm 0.013$

$0.331 \pm 0.009$

$0.330 \pm 0.009$

$0.330 \pm 0.009$
Accelerating voltage, $\mathrm{kV}$

$\eta_{E}$

10

15

0.339

0.31

0.319

Aluminum

Accelerating voltage, $\mathrm{kV}$

$\eta_{M}$

Accelerating

voltage, $\mathrm{kV}$

$\eta E$

19.8

$0.175 \pm 0.007$

10

0.171

25.1

$0.168 \pm 0.007$

30

0.155

$0.176 \pm 0.007$

35.0

$0.170 \pm 0.007$

However, for $25.1 \mathrm{kV}$ in copper, the discrepancy between calculated and experimental backscatter ratios is somewhat large. This may be due to the effect of the final step. In the present calculation, the backscattered electrons produced in each step are about $5 \%$ at $34.7 \mathrm{kV}, 6 \%$ at $30 \mathrm{kV}$, and $11 \%$ at $25.1 \mathrm{kV}$. Since the 

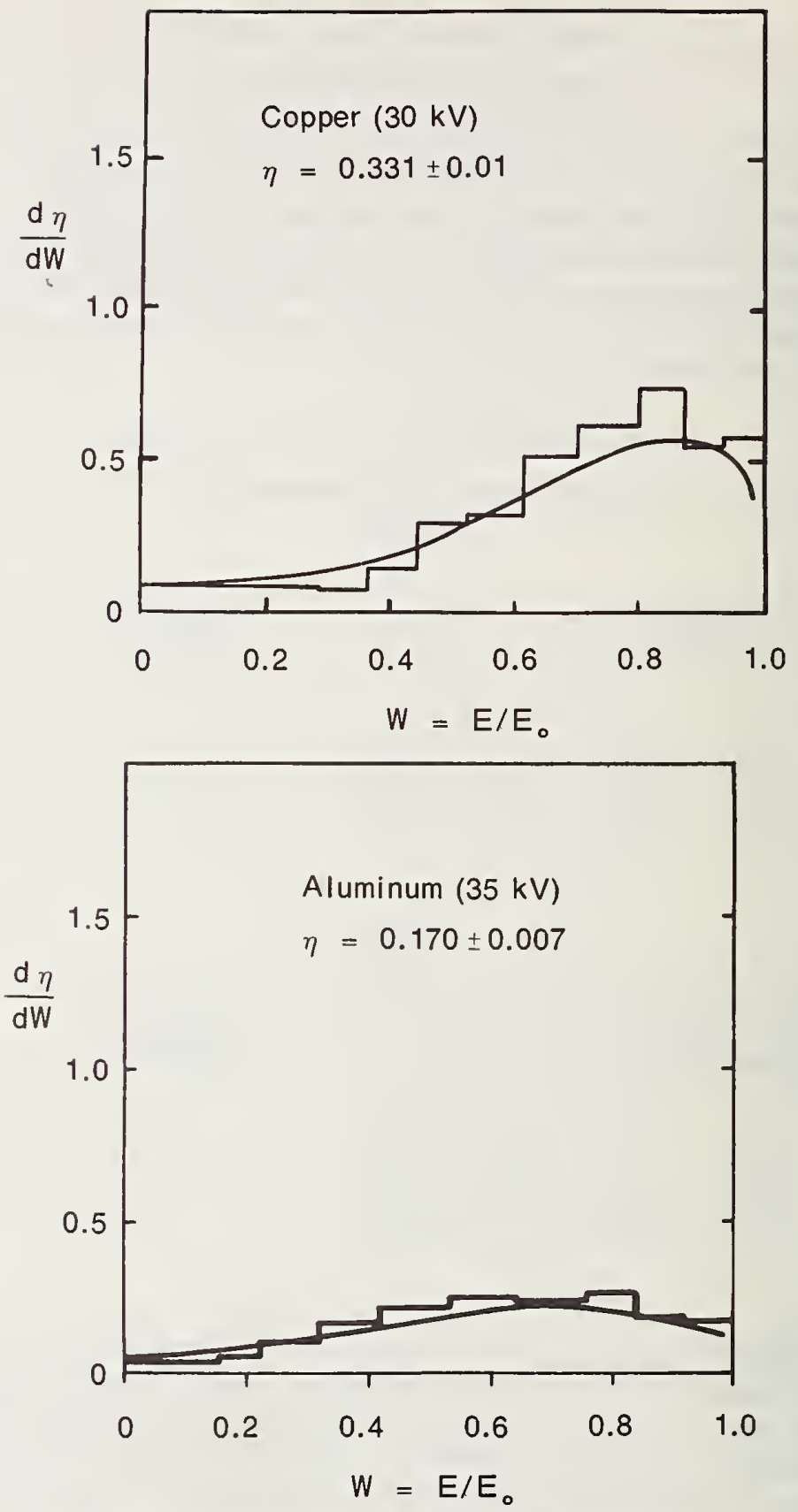

Figure 5. Energy distribution of backscattered electrons. Full curve: Kulenkampff and Spyra. Histogram: present calculation. 
energy in the final step is small, the Born approximation is no longer valid; and consequently, the error increases with the increase of the contribution of the final step.

\section{Absorption of Electrons}

Absorption curves for electrons are shown in Figure 6 together with experimental results. From these curves, the extrapolated range $\mathrm{R}$ has been calculated as shown in Table 4 .

Table 4. Extrapolated range.

$\mathrm{R}_{x}$ : by Monte Carlo calculation.

$\mathrm{R}_{K p}$ : by the experimental formula of Katz and Penfold [17]

Copper

Accelerating voltage, $\mathrm{kV}$

$$
\mathrm{R}_{x, \mu \mathrm{m}}
$$

Accelerating voltage, $\mathrm{kV}$
Aluminum

$$
\mathrm{R}_{x}, \mu \mathrm{m} \quad \mathrm{R}_{K P}, \mu \mathrm{m}
$$

2.66

4.22

5.38

7.48
2.45

7.49

Agreement between theory and experiment is good. For $\mathbf{R}_{x}$ the following experimental formulas have been obtained:

$$
\left.\begin{array}{c}
\text { Copper: } \rho \mathrm{R}_{x}=5.65 \times 10^{2} \mathrm{E}_{o}^{1.72} \\
\text { Aluminum: } \rho \mathrm{R}_{x}=8.70 \times 10^{2} \mathrm{E}_{o^{1.81}}
\end{array}\right\}
$$

in which

$$
\mathrm{E}_{\boldsymbol{o}} \text { : initial energy of electron in } \mathrm{MeV} \text {, }
$$

$$
\rho \mathrm{R}_{x}: \mathrm{mg} / \mathrm{cm}^{2} .
$$

These values are close to those obtained by Glocker [18] and those summarized by Birkoff [19]. 

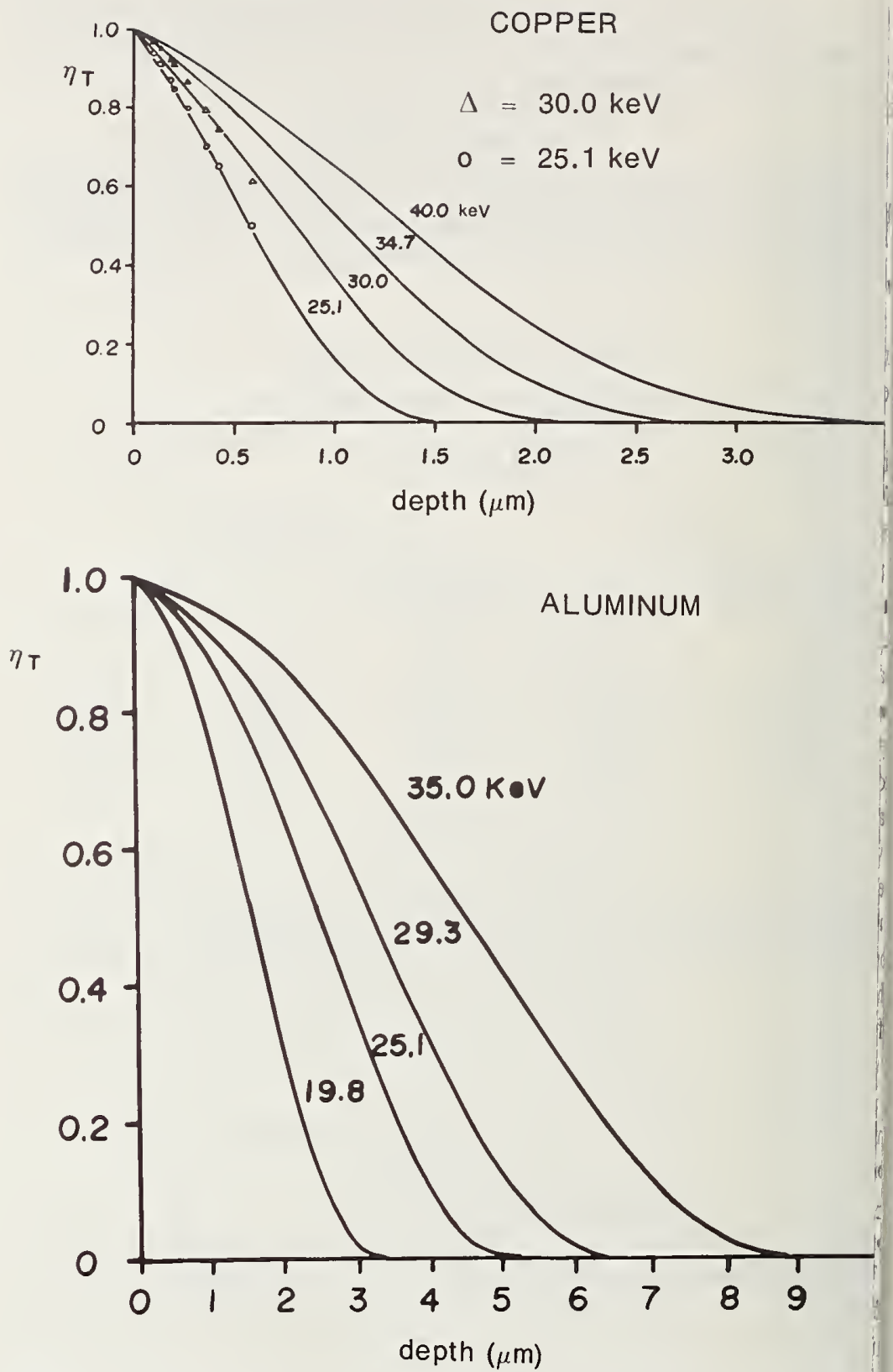

Figure 6. Absorption of electrons, copper (top), (experimental: Cosslett and Thomas), and aluminum (bottom). 


\section{DEPTH OF COMPLETE DIFFUSION}

The depth of complete diffusion, $\mathrm{x}_{d}$, is a very convenient quantity for understanding the behavior of electrons in the specimen.

However, in the actual case, the transition from a straight path to a diffused path is not abrupt; and, consequently, it is not so easy to obtain experimentally an exact value of $\mathrm{x}_{d}$.

For the physical significance of $\mathrm{x}_{d}$, the following explanations are proposed by Cosslett and Thomas [12]:

a. After many scattering events the absorption becomes exponential when a diffused state is approached. Therefore, the beginning of the exponential relation gives $\mathrm{x}_{d}$.

b. With the increase of penetration depth, the electron travel directions become divergent and, finally, the most probable angle of distribution of scattering angles becomes constant. Such a state is that of complete diffusion.

c. In the state of complete diffusion, the probabilities of scattering in forward and backward directions should be equal. However, this depth is not equal to the depth at which the number of forward traveling electrons has decreased to $1 / 2$ the initial value, since due to the effect of backscattered electrons, the number of electrons traveling in the forward direction is smaller than half the number of incident electrons; consequently the estimate of $\mathrm{x}_{d}$ from this standpoint will give too shallow values.

On the other hand, the present Monte Carlo approach will give a clearer idea. Figure 7 is the result of calculations for copper and aluminum. This figure shows the frequency of backscattered and absorbed electrons and the maximum depth of their penetrations. The right hand side corresponds to backscattered electrons and the left hand side to absorbed electrons. $\mathrm{x}_{\mathrm{x}}$ is the range of electrons.

On Figure 7, we can observe the following points:

a. Backscattered electrons are mainly scattered near the surface, and very few of them can penetrate beyond $x_{d}$, the depth of complete diffusion.

b. The highest frequency of maximum penetration depth of absorbed electrons nearly coincides with $\mathrm{x}_{d}$.

The maximum penetration depth of an electron is the depth at which the electron takes a path perpendicular, in the mean, to the initial direction of incidence. This depth is the starting point of random movement for that electron. The maximum frequency for 


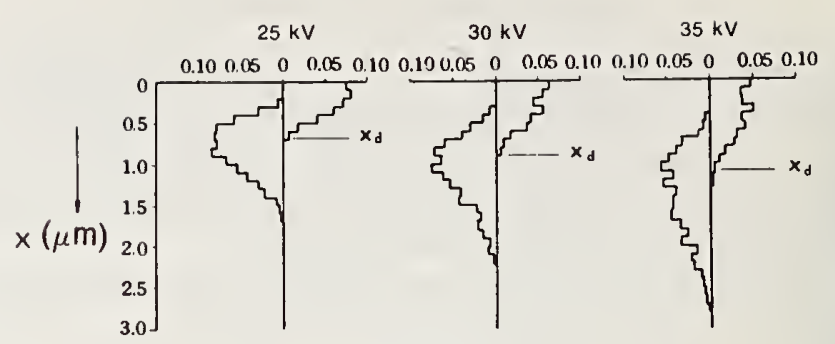

COPPER

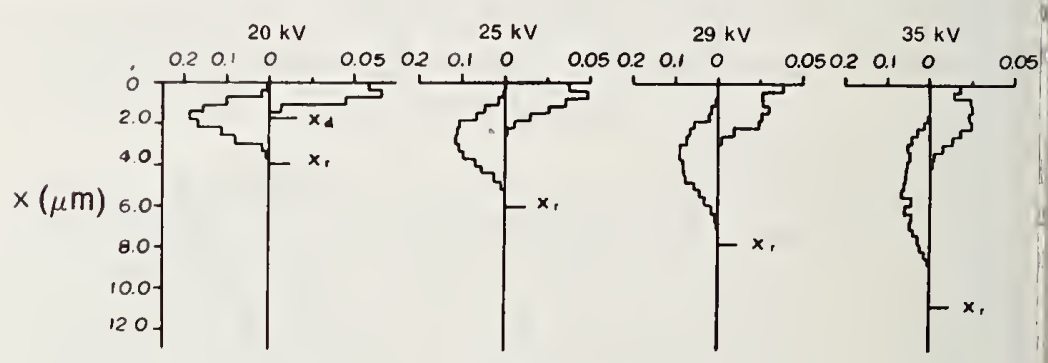

ALUMINUM

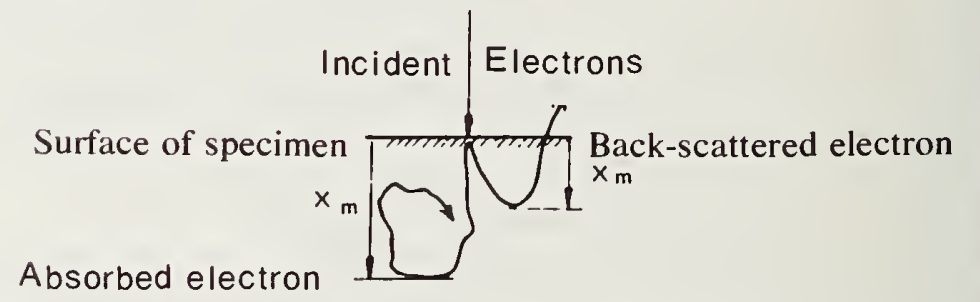

Figure 7. Distribution of maximum penetration depth for backscattered electron (right hand side) and absorbed electrons (left hand side) for copper and aluminum

the absorbed electron should occur at $\mathrm{x}_{d}$, since electrons which have traveled beyond this depth cannot escape through the surface. This last point is to be expected from the Archard diffusion model. The schematic explanations of the paths of backscattered and absorbed electrons are shown at the bottom of Figure 7. 
The diffusion process in the target is illustrated in Figure 8 , in which the depth distribution of electrons in each step is shown. Until the ninth step, the original form of the distribution is preserved; from there on, the change of wave form becomes more significant. This process occurs at the depth $\mathrm{x}_{d}$ and quite resembles a transition from a shock wave to a sound wave or from a square wave to sine wave.

\section{E. DEPTH DISTRIBUTION OF CHARACTERISTIC X-RAYS}

One method to check the validity of the above calculation is to calculate the depth distribution function of characteristic $\mathrm{x}$-rays and to compare it with the results obtained by other methods. If we denote by $\mathrm{N}_{K}$ the number of $\mathrm{K}_{\alpha}$ quanta and by $\mathrm{n}_{K}$ the total number of ionizations of the K-shell, we have

$$
\mathrm{N}_{K}=\omega \mathrm{n}_{K}
$$

where $\omega$ is the fluorescence yield showing the decrease of the number of $K_{\alpha}$ quanta due to the Auger effect and is a function of the atomic number (Compton and Allison [20]).

Let us denote the $\mathrm{K}$-shell ionization probability as $\mathrm{Q}_{K}(\mathrm{E})$, the Avogadro number as $\mathrm{N}_{A}$, and the atomic weight as $\mathrm{A}$; then $\mathrm{dn}_{K}$, the number of ionizations during the electron travel of length $\mathrm{dx}$, becomes

$$
\mathrm{dn}_{K}=\left(\mathrm{N}_{A} \rho / \mathrm{A}\right) \mathrm{Q}_{K}(\mathrm{E}) \mathrm{dx}
$$

Therefore the total number of ionizations is given by

$$
\mathrm{n}_{K}=\int \frac{\mathrm{dn}_{K}}{\mathrm{dx}} \mathrm{dx}=\int \frac{\mathrm{N}_{A} \rho}{\mathrm{A}} \mathrm{Q}_{\mathrm{K}}(\mathrm{E}) \mathrm{dx} .
$$

and $\mathrm{n}_{\kappa}$ can be calculated if $\mathrm{Q}_{\kappa}(\mathrm{E})$ and $\omega$ are known.

However, since the distribution function is only related to relative values, i.e. the relative change of $x$-ray generation with depth, it is not necessary to know the absolute value of $\omega$.

On the other hand, $\mathrm{Q}_{\kappa}(\mathrm{E})$ is a function of energy, for which Mott and Massey [21] obtained the following formula using Bethe's nonrelativistic treatment:

$$
\mathrm{Q}_{\kappa}(\mathrm{E})=\left(2 \pi \mathrm{e}^{4} / \mathrm{E}_{\kappa}\right) \cdot(1 / \mathrm{E}) \mathrm{b} \ln (4 \mathrm{E} / \mathrm{B})
$$



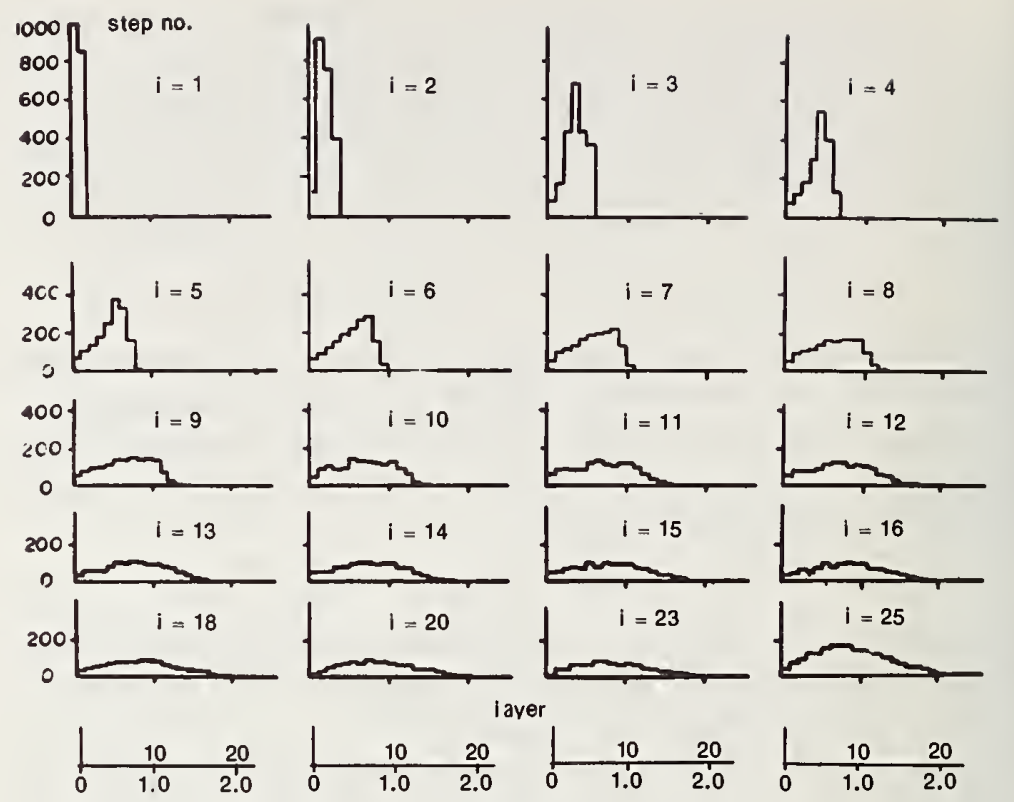

$\operatorname{depth}(\mu \mathrm{m})$

Figure 8. Diffusion process in the target. Frequency as a function of depth $-\mathrm{i}$ $=$ number of steps.

Horizontal scaler: Number of layers (upper scale), and depth in $\mu \mathrm{m}$ (lower scale).

if we accept for $\mathbf{b}$ and $\mathbf{B}$, Worthington and Tomlin's [22] values,

$$
\mathrm{b}=0.35 \text { and } \mathrm{B}=[1.65+2.35 \exp (1-\mathrm{U})] \cdot \mathrm{E}_{\kappa},
$$

we have

$$
\mathrm{Q}_{K}(\mathrm{E}) \cdot \mathrm{E}_{K}^{2}=0 \cdot 7 \pi \mathrm{e}^{2} \frac{1}{\mathrm{U}} \ln \frac{4 \mathrm{U}}{1 \cdot 65+2 \cdot 35 \exp (1-\mathrm{U})}
$$

where $\mathrm{E}_{\kappa}$ is the excitation energy of the $\mathrm{K}$-shell and $\mathrm{U}=\mathrm{E} / \mathrm{E}_{\kappa}$.

Figure 9 shows a comparison of the theoretical and experimental values, (Pockman et al. [23]), for nickel together with corrected values considering relativistic effects (Perlman [24]). For nickel, since $\mathrm{E}=80 \mathrm{keV}$ for $\mathrm{U}=10$, the effect of the relativistic correction is 


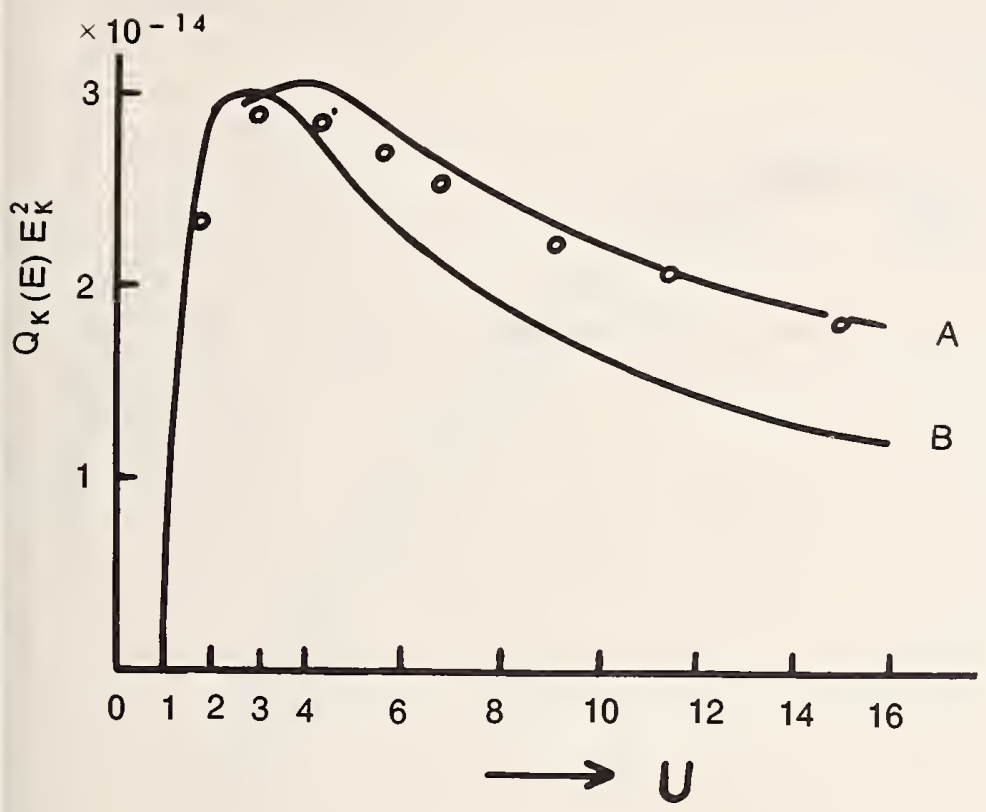

Figure 9. Ionization cross section of K-shell for nickel.

A. Relativistic theory of Perlman.

B. Non-relativistic theory of Worthington and Tomlin.

$\mathrm{o}=$ experimental result of Pockman et al.

no longer negligible; therefore, in the case of copper, the experimental value of Pockman et al. [23] has been used in the following relation

$$
\left[\mathrm{Q}_{K}(\mathrm{E}) \cdot \dot{\mathrm{E}_{\kappa}}{ }^{2}\right]_{C u}=\left[\mathrm{Q}_{\kappa}(\mathrm{E}) \cdot \dot{\mathrm{E}}^{2}\right]_{N i}
$$

However, in aluminum, even for $U=20, E=30 \mathrm{keV}$ and, therefore, a non-relativistic treatment has been adopted.

The depth distributions are summarized for total electrons, dividing the target into layers of $0.05 \mu \mathrm{m}$ for copper and $0.1845 \mu \mathrm{m}$ for aluminum. Results for copper are shown in Figures 10 and 11. To compare these results with those of Castaing and Descamps [25] for $29 \mathrm{kV}$, it should be normalized by

$$
\cdot \frac{\left[\int \phi_{M}(\rho \mathrm{z}) \mathrm{d} \rho \mathrm{z}\right]_{E=E \mathrm{keV}}}{\left[\int \phi_{T}(\rho \mathrm{z}) \mathrm{d} \rho \mathrm{z}\right]_{29 \mathrm{keV}}}=\frac{\left[\left(\mathrm{E}-\mathrm{E}_{K}\right)^{1.7}\right]_{E=E \mathrm{keV}}}{\left[\left(\mathrm{E}-\mathrm{E}_{K}\right)^{1.7}\right]_{29 \mathrm{keV}}}
$$




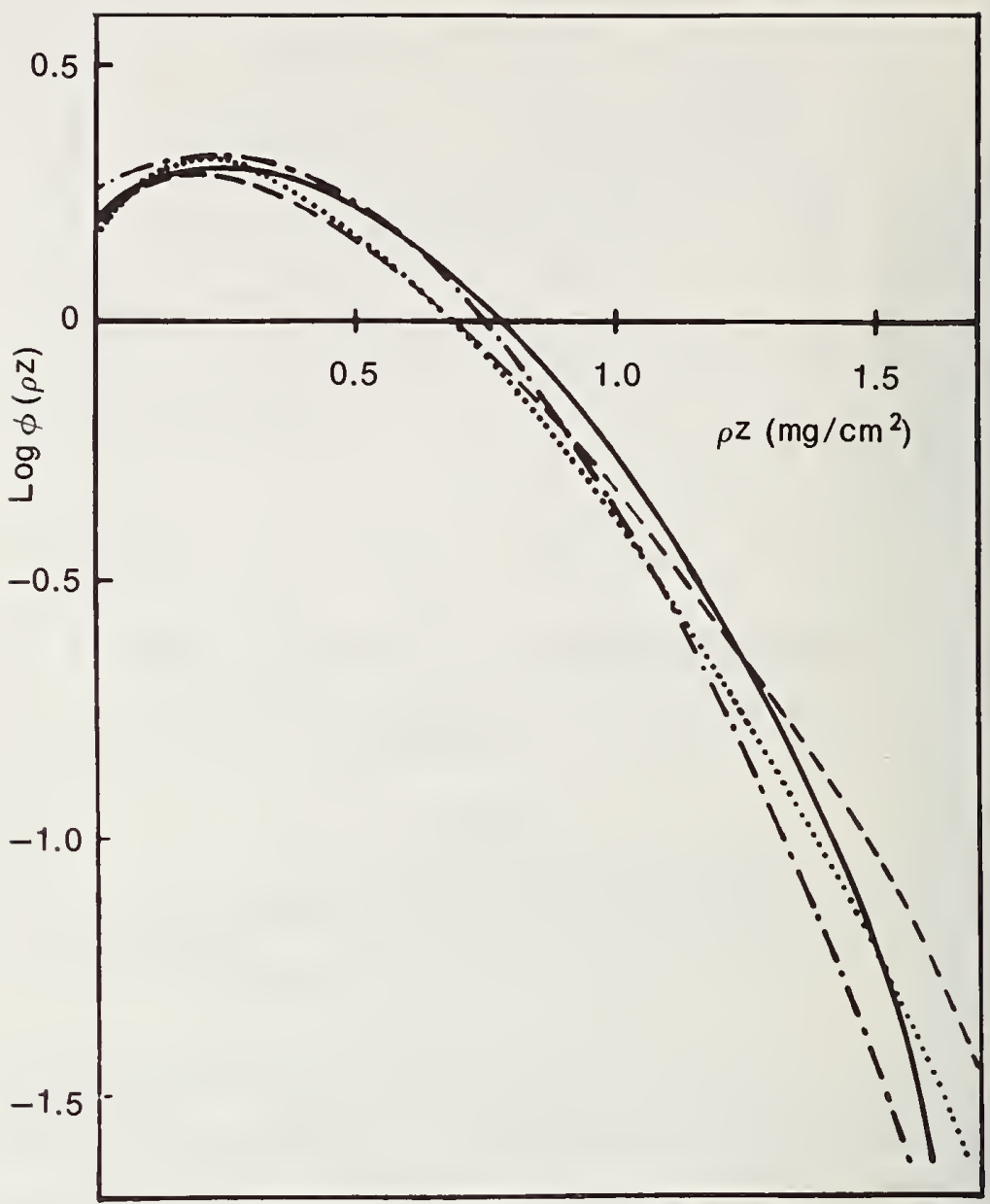

Figure 10. A comparison of the calculated depth distribution of CuK radiation with the results of the other two methods.

Monte Carlo calculation $\left(30 \mathrm{kV}, \gamma=90^{\circ}\right)$.

- - Tracer method $\left(28.5 \mathrm{kV}, \gamma=80^{\circ}\right)$.

-..- Analysis of angular distribution $\left(30 \mathrm{kV}, \gamma=90^{\circ}\right)$.

. Castaing and Deschamps $\left(29 \mathrm{kV}, \gamma=80^{\circ}\right)$.

$\gamma=$ incident angle. 


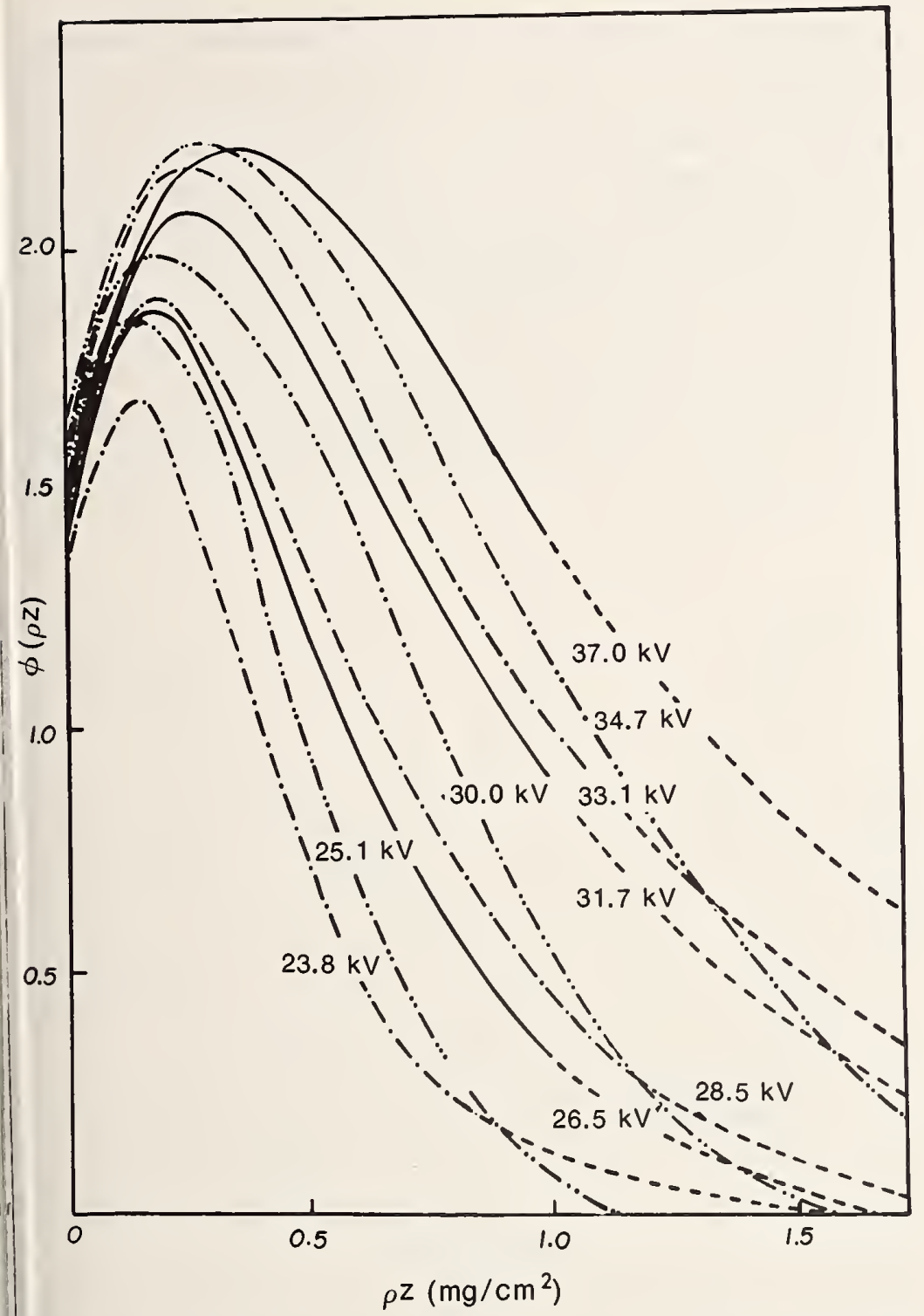

Figure 11. Depth distribution of $\mathrm{CuK} \alpha$ radiation at various accelerating voltages.

- ARL-EPMA, take-off angle : $52.5^{\circ}$.

-...- JXA-3 XMA, take-off angle: $15^{\circ}$.

-.....- Monte Carlo method. 
where $\phi_{T}(\rho \mathrm{z})$ and $\phi_{M}(\rho \mathrm{z})$ are those obtained by Castaing and Descamps, and by the present Monte Carlo calculation, respectively.

Figure 12 shows the depth of maximum $x$-ray generation in copper and aluminum. The variation of this depth depends on the accelerating voltages, and the maximum nearly coincides with the depth at which electron absorption just begins. This means that in $\mathrm{x}-$ ray generation, the role of inelastic scattering of electrons near the surface is very important. Following the Archard diffusion model, the maximum depth of $\mathrm{x}$-ray generation, $\mathrm{x}_{m}$. should coincide with $\mathrm{x}_{d}$. The distribution function derived from the Archard model shows better agreement with experiment than that from a straight line course model. But even for the Archard model, the value of the peak is too deep.

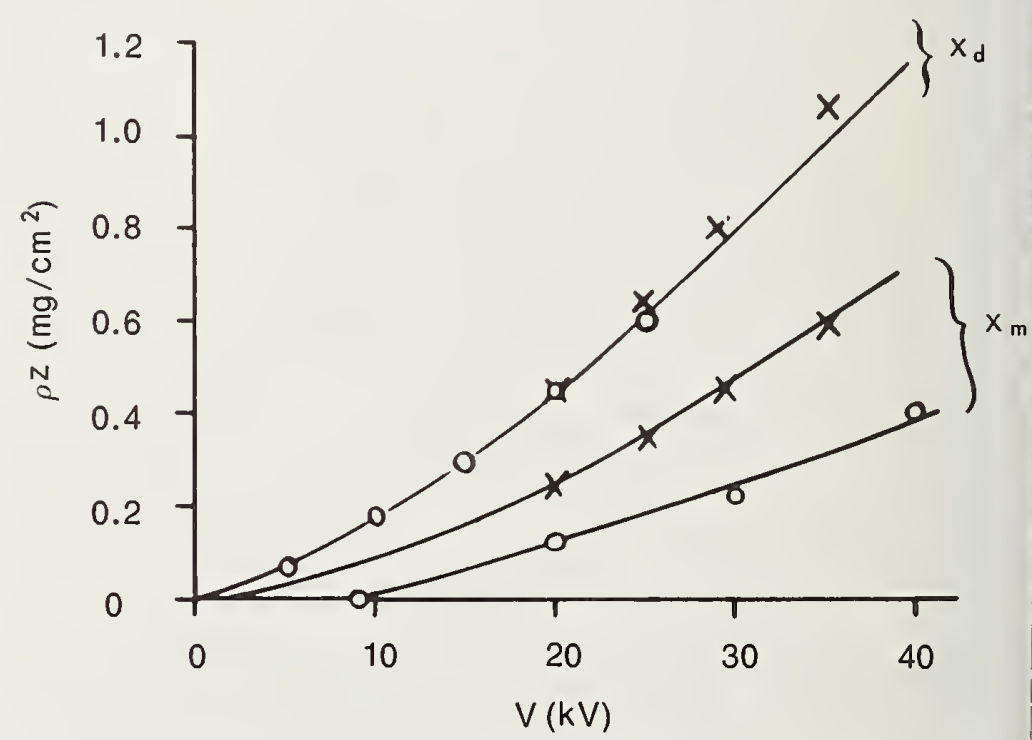

Figure 12. Depth of maximum $\mathrm{x}$-ray generation $\mathrm{x}_{m}$ and complete diffusion $\mathrm{x}$

$$
\begin{aligned}
\rho \mathrm{z} & =\text { depth }, \mathrm{V}=\text { accelerating voltage; } \\
\mathrm{O} & =\text { copper and } \mathrm{x}=\text { aluminum. }
\end{aligned}
$$


Figure 13, shows the distribution function for aluminum obtained by the Monte Carlo calculation, and in Figure 14 results obtained by analysis of the angular distribution are shown together with that of the tracer method of Hénoc [26] for comparison. Compared with the other two methods, the Monte Carlo calculation gives less significant $\mathrm{x}$-ray generation in a deep layer. However, as the range of electrons at $30 \mathrm{keV}$ is $2.1 \mathrm{mg} / \mathrm{cm}^{2}$, generation of $\mathrm{x}$-rays in a range deeper than this depth should not be considered primary excitation.

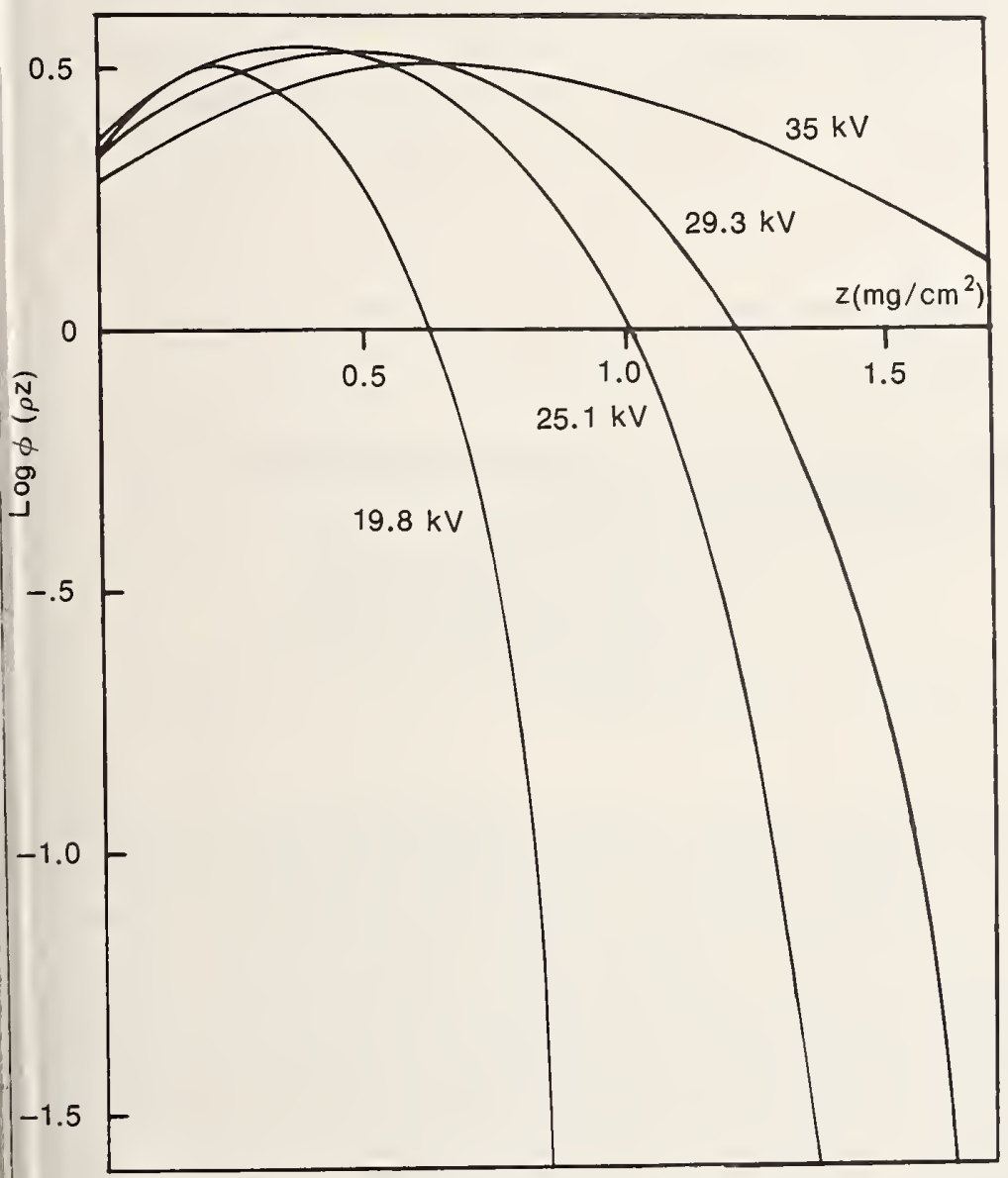

igure 13. Depth distribution of $\mathrm{AlK} \alpha$-radiation at various accelerating voltages. 


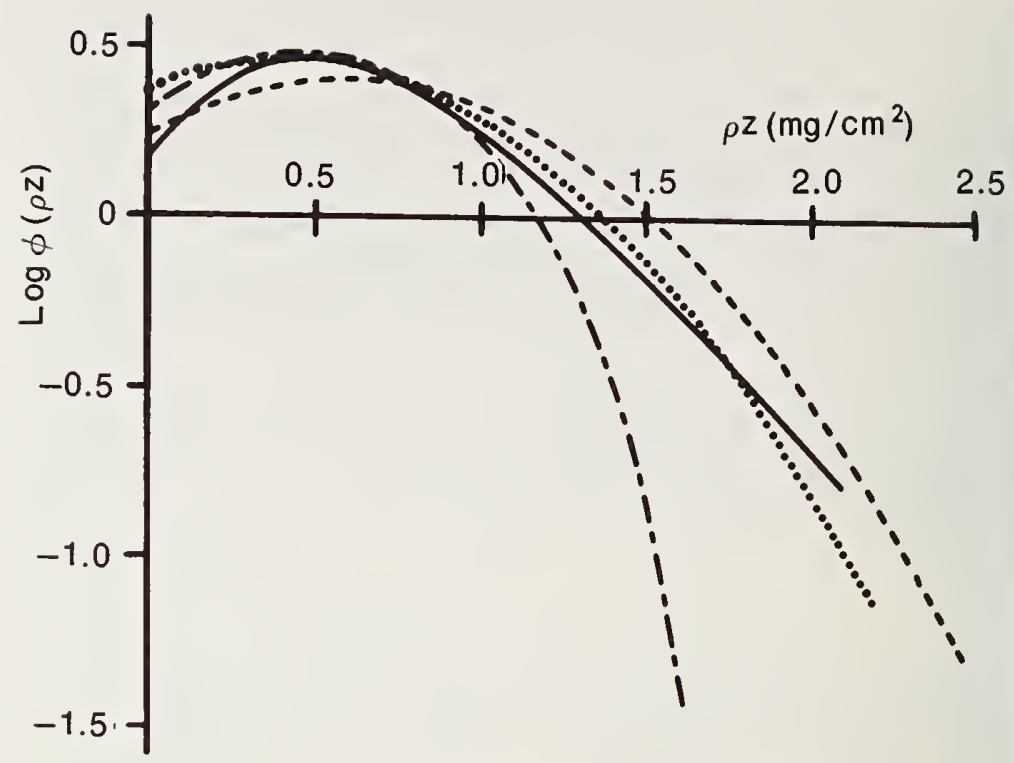

Figure 14. A comparison of the calculated depth distribution of AlK $\alpha$-radiatio with the results of the other two methods.

Analysis of angular distribution, $(30 \mathrm{kV})$.

- Tracer method (Henoc), (29 kV).

-..-.-- Monte Carlo calculation, $(29.3 \mathrm{kV})$.

$-----\mu / \rho=385.7$

…... $\mu / \rho=459$

In the tracer method of Hénoc, where magnesium is used as a tracer, excitation of $\mathrm{Mg} \mathrm{K}_{\alpha}$ by $\mathrm{Al}$ radiation would be plausible. Also, in the angular distribution analysis of characteristic rays, excitation due to continuous and characteristic x-rays from heavier impurity elements would be conceivable. Therefore, one should recall that our Monte Carlo calculation will give the distribution function for primary $\mathrm{x}$-ray generation, while the angular distribution analysis gives that for actual analyzing conditions in the electron probe microanalyzer. However, the difference is mainly in the lower intensity region and, therefore, either value will be usable for the actual correction. 
In the numerical computation of corrections, the $\mathrm{f}(\chi)$-function, as hown in Figure 15, is usually used. The present Monte Carlo alculation gives somewhat larger $\mathrm{f}(\chi)$ values; however, the lifference is not very significant. Green's results [27] are also hown, marked by circles.

In the analysis of the angular distribution of characteristic x-rays, xact values of absorption coefficients are indispensable. In Figure 4, two results for different absorption coefficients, one an xperimental value of Andrews [28], the other a computed value of Heinrich [29], are shown. The higher value gives a better result. Recently, a new value has been reported by Bucklow [30], Metals Research Limited; this value is between the previous two $(\mu / \rho=408$ $\mathrm{m}^{2} \mathrm{gm}^{-1}$ ). It gives better agreement with those obtained by other nethods, especially in the high intensity region.

\section{F. SHAPE AND SIZE OF THE X-RAY SOURCE}

To determine the size of the $x$-ray source, it is important to obtain an exact idea of the resolving power of the electron probe microanalyzer. Ehrenberg and King [31] investigated the electron diffusion in targets using fluorescent materials. However, this does ot coincide with the diffused $\mathrm{x}$-ray source, because primary $\mathrm{x}$-ray ources should be smaller than the electron diffusion zone. On the ther hand, for an element with high atomic number, the fluorescent k-radiation excited by the continuum is significant; and onsequently, the x-ray source will broaden and become larger than he electron diffusion zone. In our laboratory, the size and shape of he $x$-ray source was determined in copper using the following nethods:

a. from a relation between $\mathrm{x}$-ray intensity and knife edge displacement-source broadening,

b. from the Monte Carlo calculation - source depth, and

c. from the depth distribution - source shape.

The source broadening determined by Shimizu and Shinoda [32] s based on the following assumptions:

a. the intensity distribution of an electron beam is of the Gaussian type.

b. the functional representation of a wedge-shaped specimen raversing the path of the electron beam is expressed by a stepunction, and 


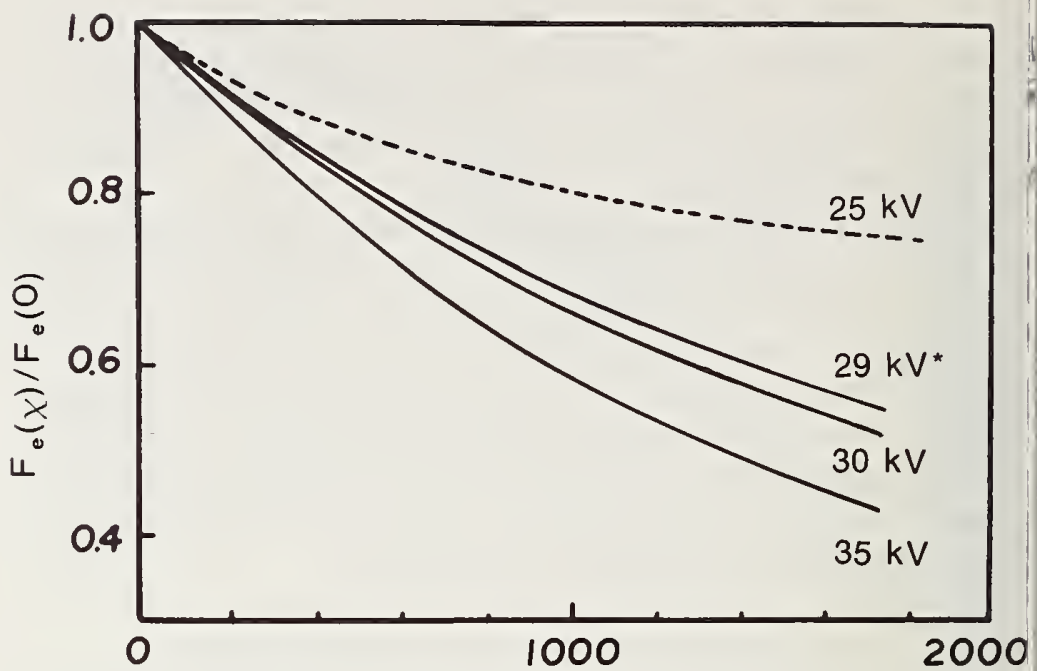

(a) COPPER $\quad \chi\left(=\frac{\mu}{\rho} \operatorname{cosec} \theta\right)$

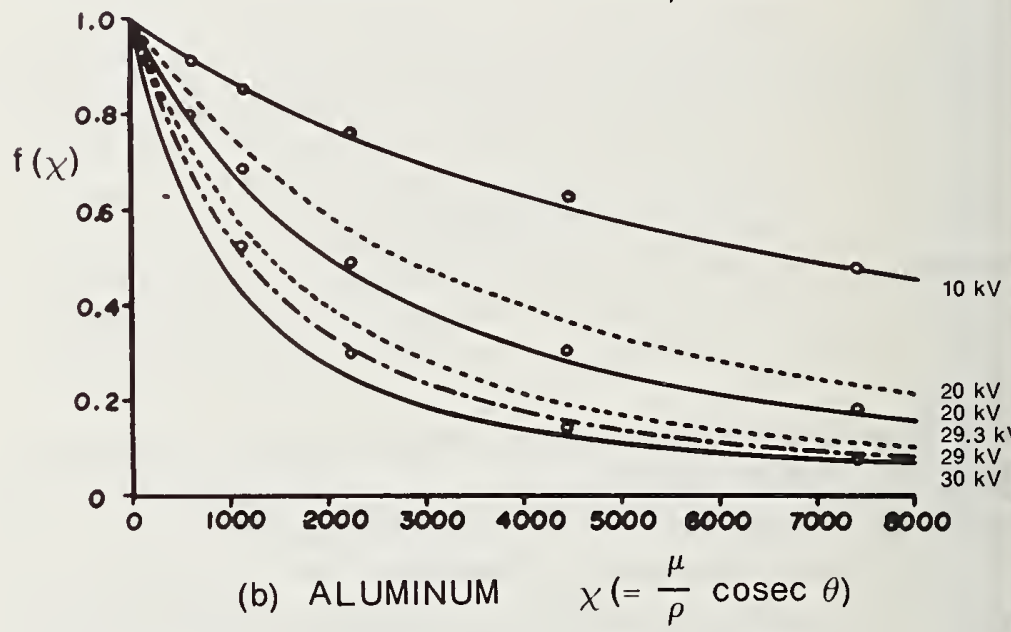

Figure 15. Relation between $\mathrm{f}(\chi)$ and $\chi(=\mu / \rho \operatorname{cosec} \theta)$.

(a) Copper, from angular distribution.

(b) Aluminum, from comparison of various methods.

- Angular distribution of $\mathrm{x}$-ray.

- - Monte Carlo calculation.

-.-.- Tracer method (Hénoc, 1966).

o Green $(9.8,20.5,29 \mathrm{kV})$. 
c. the intensity distribution of $\mathrm{x}$-rays in a lateral direction for an electron beam with infinitesimal cross section is given by a Gaussian.

The excitation by the continuum is neglected, because it is less than $10 \%$. The result is as shown in Figure 16.

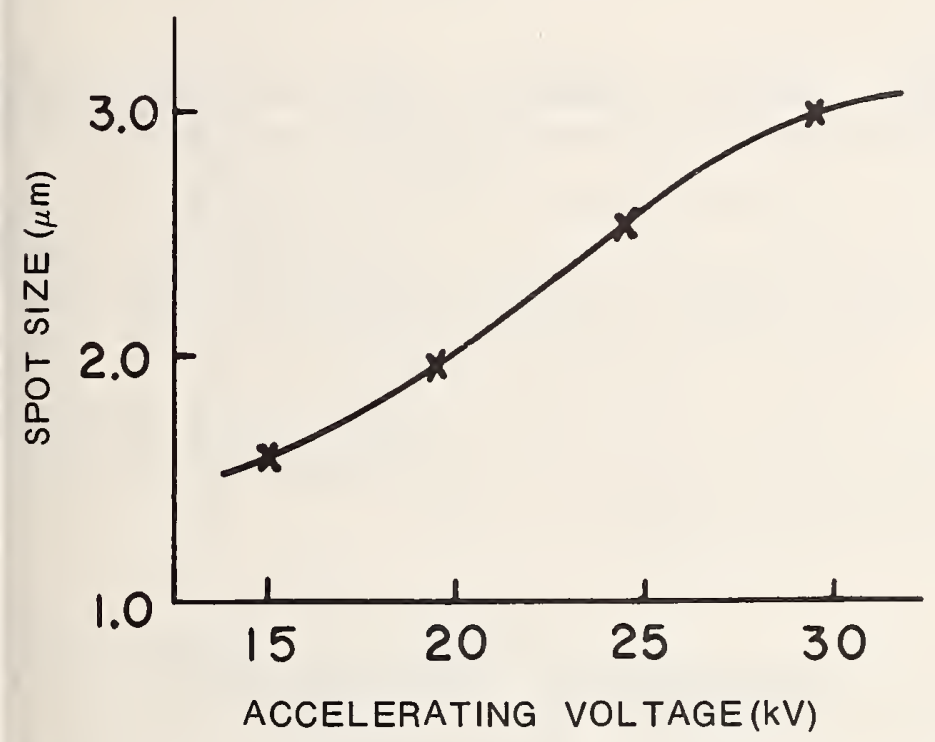

Figure 16. Variation of size of the diffused $x$-ray source for copper with accelerating voltage, the electron beam diameter being $1 \mu \mathrm{m}$.

If the intensity distribution in the direction of depth is also expressed by a Gaussian ${ }^{2}$, the shape of the $x$-ray source is given by Wittry [33]. ${ }^{3}$

$$
\mathrm{F}(\mathrm{x}, \mathrm{y}, \mathrm{z})=\mathrm{F}(\mathrm{z}) \cdot \mathrm{F}(\mathrm{x}, \mathrm{y})=\mathrm{A} \text { exp-a }\left(\mathrm{z}-\mathrm{z}_{\theta}\right)^{2} \exp -\mathrm{b}\left(\mathrm{x}^{2}+\mathrm{y}^{2}\right)
$$

Therefore, the integration of $F(x, y, z)$ with respect to $x$ and $y$ gives the distribution function $F(z)$, and for $z=0$, it becomes $\phi(0)$. Thus,

${ }^{2}$ The same assumption has been successfully applied in the case of analysis of the angular distribution of $\mathrm{x}$-rays.

${ }^{3}$ Wittry's formula, slightly modified. 
we have

$$
[\mathrm{F}(\mathrm{z})]_{z=o}=\left[\int \mathrm{F}(\mathrm{x}, \mathrm{y}, \mathrm{z}) \mathrm{dxdy}\right]_{z=o}=\phi(0)
$$

and

$$
\mathrm{A}=\frac{\mathrm{b} \phi(0)}{\pi}
$$

Figure 17 shows the shape of the diffused $x$-ray source, contourst of which are given by the following logarithmic scale, taking $y=0$

$$
\log \mathrm{F}(\mathrm{x}, \mathrm{y}, \mathrm{z})=\log \mathrm{A}-\left[\mathrm{a}\left(\mathrm{z}-\mathrm{z}_{o}\right)^{2}+\mathrm{bx} \mathrm{x}^{2}\right] \log \mathrm{e}
$$

The right hand side shows intensity contours while the left hanc side is plotted for each accelerating voltage and gives contours for

$$
F(x, 0, z)=(1 / e)[\text { Maximum value of } F(x, 0, z)]
$$

For all practical purposes, $\mathrm{x}$-ray microanalysis is done of th volume bounded by the contour of the left hand side of Figure 17 Table 5 shows these volumes for copper.

Table 5. Volume microanalyzed.

Accelerating voltage Volume analyzed

$\begin{array}{ll}\mathrm{kV} & (\mu \mathrm{m})^{3} \\ & \\ 20 & 1.38 \\ 25 & 2.97 \\ 30 & 5.56\end{array}$

Therefore, if the accelerating voltage is reduced $20 \%$ from $30 \mathrm{kV}$ the volume used for microanalysis is reduced by half, and we wil obtain information from a much smaller volume.

\section{G. RESOLVING POWER IN SPECIMEN CURRENT ANALYSIS}

As already shown, the maximum depth of backscattered electron does not exceed $\mathrm{x}_{d}$; and the rate of backscattering is determine by the mean atomic number of the material between the surfac and $\mathrm{x}_{d}$. Therefore, we have (specimen current)= (incident beai current) - (backscattered electron current).

However, the effect of secondary electron emission is not cor tained in the above expression. 


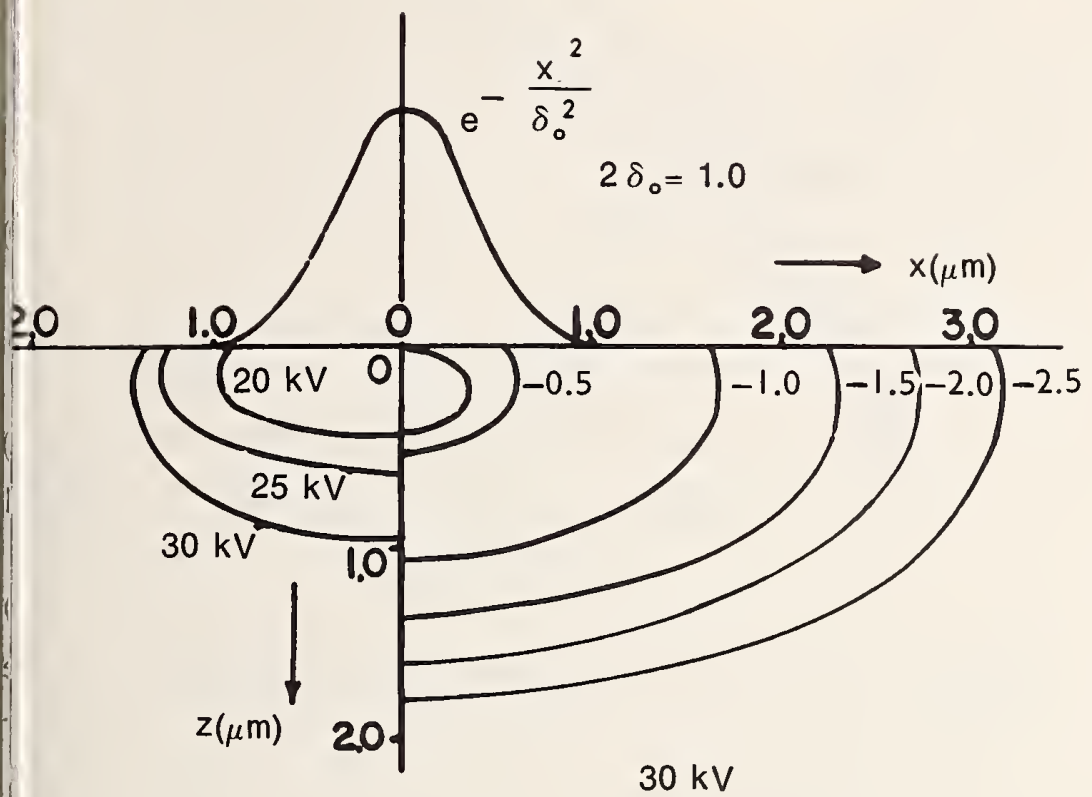

Figure 17. Shape of the $x$-ray source for copper.

Upper: intensity distribution of the electron beam.

Lower: intensity contours, left: for 1/e of maximum intensity; right: of $\mathrm{x}$-ray intensity for $30 \mathrm{kV}$ on a logarithmic scale.

The resolving power of the specimen current method should be etermined by the behavior of electrons between the surface and the epth $\mathrm{x}_{d}$. Figure 18 shows fractions of electron backscattering and -ray generation as a function of specimen thickness. The depths for $0 \%$ are $0.92 \mu \mathrm{m}$ for $\mathrm{x}$-rays and $0.61 \mu \mathrm{m}$ for backscattered elecfons. If electron scattering occurs according to Archard's model nd the shape of the $\mathrm{x}$-ray source is as shown in Figure 17, the olume analysed by $x$-rays is about five times that of backscattered lectrons.

Practically, this ratio will be much larger because if the theory f Bethe et al. [5] is valid to the depth $\mathrm{x}_{d}$, the mean deviation $\theta$ rom the initial direction is given by

$$
\langle\cos \theta\rangle_{a v}=(1 / \mathrm{e}) \text {. }
$$




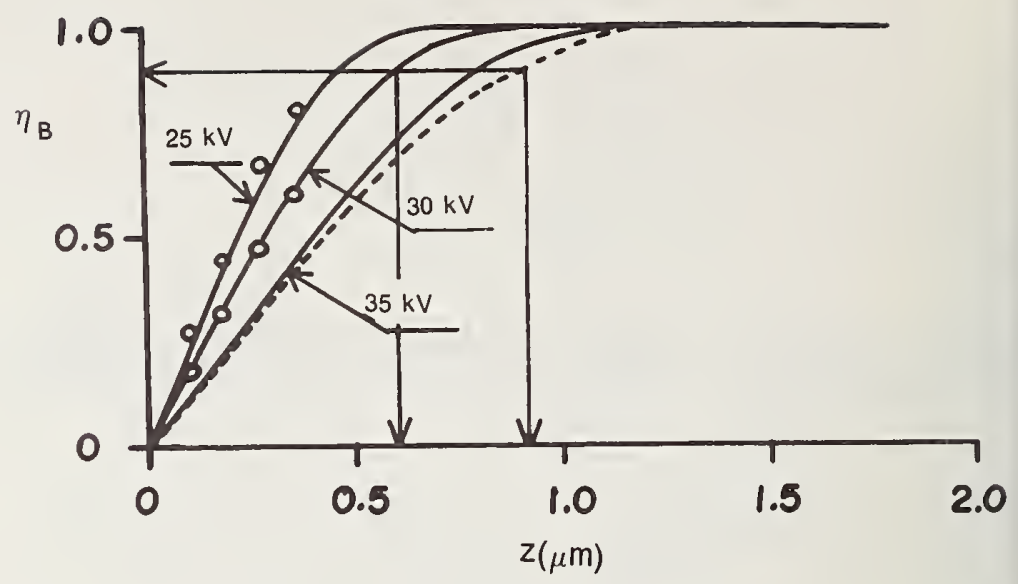

Figure 18. Fractional backscattering of electrons $\eta_{\mathrm{B}}$ and emission of $\mathrm{x}$-rays for copper.

- X-ray $(30 \mathrm{kV})$.

o (Cosslett and Thomas, 1965).

This means that at a depth close to $\mathrm{x}_{d}$, the deviation reaches this level; and, therefore, until certain depths, no appreciable deviation of electron travel direction should occur. Hence, the volume analysed by the backscattered electron or absorbed electron methods should not exceed signficantly the volume determined by the beam cross scetion times $x_{d}$. This volume should be much smaller than that calculated from Archard's model.

This paper is based mainly on the Thesis (Osaka University. 1967 ) by K. Murata, one of the authors. 


\section{References}

[1] Shimizu, R., Kishimoto, H., Shirai, T., Murata, K., Shinoda, G., and Miura, M., Technol. Repts., Osaka Univ. 16, 415 (1966).

[2] Murata, K., Shimizu, R., and Shinoda, G., Technol. Repts., Osaka Univ. 16, 121 (1966).

[3] Shimizu, R., Murata, K., and Shinoda, G., Technol. Repts., Osaka Univ. 17, 13 (1967).

[4] Archard, G., J. Appl. Phys.32, 1505 (1961).

[5] Bethe, H. A., Rose, M. E., and Smith, L. P., Proc. Amer. Phil. Soc. 78, 573 (1938).

[6] Lewis, H. W., Phys. Rev. 78, 526 (1950).

[7] Goudsmit, S., Saunderson, J. L., Phys. Rev. 57, 24 (1940).

[8] Wentzel, G., Z. Phys. 40, 590 (1927).

[9] Kulchitsky, L. A., and Latychev, G. D., Phys. Rev. 61, 254 (1942).

[10] Moliere, G., Z. Naturforsch. 3a, 78 (1948).

[11] Nigam, B. P., Sundaresan, M. K., and Wu, Ta-You, Phys. Rev. 115, 491 (1959).

[12] Cosslett, V. E., and Thomas, R. N., Brit. J. Appl. Phys. 15, 235 (1964); 15, 883 (1964); 15, 1283 (1964).

[13] Cosslett, V. E., and Thomas, R. N., Brit. J. Appl. Phys.16, 779 (1965).

[14] Wilson, R., Phys. Rev. 60, 749 (1964).

[15] Kulenkampff, H., and Spyra, W., Z. Phys.137, 416 (1954).

[16] Burkhalter, P. G., Bureau Mines Rept. Invest. 6681 (1965).

[17] Katz, L., and Penfold, A. S., Rev. Mod. Phys. 24, 28 (1952).

[18] Glocker, R., Z. Naturforsch. A3, 147 (1938).

[19] Birkoff, R. D., Handb. d. Phys. 34, 135, Springer, Berlin (1958).

[20] Compton, A. H., and Allison, S. K., X-Ray in Theory and Experiment, Van Nostrand, New York (1935).

[21] Mott, N. F., and Massey, H. S. W., Theory of Atomic Collision, Oxford Univ. Press, New York (1949).

[22] Worthington, C. R., and Tomlin, S. G., Proc. Phys. Soc. 69, 401 (1956).

[23] Pockman, L. T., Webster, D. L., Kirkpatrick, P., and Harworth, K., Phys. Rev. 71, 330 (1947).

[24] Perlman, H. S., Proc. Phys. Soc. 76, 623 (1961).

[25] Castaing, R., and Descamps, J., J. Phys. Radium 16, 304 (1955).

[26] Hénoc, J., J. Microscope 5, 122 (1966).

[27] Green, M., Proc. Phys. Soc. 83, 435 (1964).

[28] Andrews, C. L., Phys. Rev. 54, 994 (1938).

[29] Heinrich, K. F. J., The Electron Microprobe, eds. T. D. McKinley, K. F. J. Heinrich and D. B. Wittry, J. Wiley, New York (1966).

[30] Bucklow, I. A., Private Communication.

[31] Ehrenberg, W., and King, D. E. N., Proc. Phys. Soc. 81, 751 (1963).

[32] Shimizu, R., and Shinoda, G., Technol. Repts., Osaka Univ. 14, 897 (1964).

[33] Wittry, D. B., J. Appl. Phys. 29, 1543 (1958). 


\section{APPENDIX}

Scattering in a Coulomb field produced by a single charge is described by the well-known Rutherford scattering formula. However, in an atomic field, the screening effect of the electron cloud should be considered. If an appropriate atomic field is given, these calculations become possible. Using a function $\mathrm{g}(\mathrm{y})$, the single scattering cross section $\sigma(\theta)$ may be expressed by

$$
\sigma(\mathrm{y})=\frac{\mathrm{Z}^{2} \mathrm{e}^{4}}{\mathrm{p}^{2} \mathrm{v}^{2}} \cdot \frac{\mathrm{g}(\mathrm{y})}{\mathrm{y}^{4}}
$$

where

$$
y=\sin (\theta / 2)
$$

For $g(y)=1$, Eq. (1) gives the Rutherford scattering formula. To estimate the screening effect, the screening angle $\chi_{\alpha}$ should be defined as follows, (Goudsmit and Saunderson [7]):

$$
\ln \frac{1}{\chi_{a}}-\frac{1}{2}=\int_{0}^{1} \frac{g(y)}{y} d y
$$

If we use the Born approximation and denote the scattering cross section by $\sigma_{B}$, the screening angle $\chi_{0}$ is obtained by substituting, $\mathrm{g}_{B}\left(=\sigma_{B} / \sigma_{R}\right)$ into Eq. (2), $\sigma_{B}$ being the Rutherford scattering cross section. Two examples of the atomic field are given:

$$
\begin{gathered}
\text { 1. Exponential field } \\
\bar{V}(\mathrm{r})=\left(\mathrm{z} \mathrm{Z}^{2} \mathrm{e}^{2} / \mathrm{r}\right) \exp \left(-\lambda_{\mathrm{o}} \mathrm{r}\right)
\end{gathered}
$$

$\mathrm{g}(\mathrm{y})\left(=\mathrm{g}_{B}\right)$ is found by the Born approximation and

$$
g(y)=\frac{y^{4}}{y^{2}+y_{o}^{2}}
$$

From Eq. (2), $\chi_{o}$ becomes

$$
\chi_{o}=\mathrm{h} \lambda_{o} / \mathrm{p}=2 \mathrm{y}_{0}
$$

where

$$
\lambda_{0}=Z^{1 / 3} / \mathrm{a}
$$


Therefore, the screening parameter $\beta$ is

$$
\beta=\frac{1}{4} \chi_{o}^{2}
$$

Since many discrepancies exist among the theoretically obtained values, $\lambda_{o}$ should be modified by comparison with experimental values.

Introducing a constant $\mu$, Nigam et al. [11] have proposed that

$$
\lambda=\mu \lambda_{o}
$$

As stated already, for $20 \mathrm{keV}$ in copper, $\mu$ should be 1.12 .

2. Thomas-Fermi field

$$
\nabla(\mathrm{r})=-\frac{\mathrm{z} Z^{2}\left(\mathrm{e}^{2}\right)}{\mathrm{r}} \omega\left(\mathrm{r} \lambda_{\mathrm{o}}\right)
$$

where

$$
\lambda_{o}=Z^{1 / 3} / 0.885 \mathrm{a}_{0}
$$

By numerical computation, we have

$$
\chi_{o}^{\prime}=1.12 \mathrm{~h} \lambda_{o} / \mathrm{p}
$$

Hence, we have reached the same conclusion, and this formula gives good results, as shown already.

In our calculation, an exponential field has been adopted; but for the screening angle, we have reached the same conclusion as that deduced from the Thomas-Fermi field. Eq. (5) was first given by Wentzel [8], where, $\mathrm{a}=\mathrm{a}_{0}$ was taken as the value of $\mathrm{a}$ in $\mathrm{y}_{0}$. But as shown above, $\mathrm{a}=0.885 \mathrm{a}_{0}$ should be taken to obtain the proper value of the screening parameter. In a paper read by Bishop at the Paris Conference of X-ray Optics and Microanalysis, 1965, a similar Monte Carlo calculation can be found. However, for the screening parameter he did not use a modified one but that given by Wentzel. 



\title{
ABSORPTION EDGE EFFECTS IN ELECTRON PROBE ANALYSIS
}

\author{
D. NAGEL \\ Naval Research Laboratory, Washington, D.C.
}

\section{Abstract}

The numerical value of the mass absorption coefficient to use in calculating corrections is uncertain when an x-ray emission line used in electron probe analysis overlaps the absorption edge fine structure of another element in the specimen. The $\mu / \rho$ values normally tabulated can be in error by $\pm 50 \%$ near an edge and $\pm 10 \%$ up to $400 \mathrm{eV}$ above the edge. Test calculations of absorption and fluorescence corrections show that an uncertainty in $\mu / \rho$ of $50 \%$ commonly leads to relative errors of $1-3 \%$ in the composition. In some cases the errors exceed $10 \%$. The possible existence of such line-edge interferences can be checked for a particular specimen using standard $\mathrm{x}$-ray tables. A different $\mathrm{x}$-ray line should be used if such an interference is found. When this is not possible, calculations using a range of $\mu / \rho$ values within the expected uncertainty will show the error in the concentration which might occur.

\section{Introduction}

Calculation of composition from measured relative $\mathrm{x}$-ray intensities requires accurate knowledge of the mass absorption coefficient in electron probe analysis. It has been emphasized that uncertainties in the $\mu / \rho$ values used lead to errors in the resulting composition [1]. Uncertainties in the mass absorption coefficients arise from two sources: (a) inaccuracies in the measured, interpolated, or extrapolated values, and (b) errors in tabulations due to not including variations in $\mu / \rho$ near absorption edges. It is the purpose of this paper to examine the situation where an $x$-ray line used for analysis overlaps the absorption edge fine structure, leading to uncertainty in the $\mu / \rho$ value at the position of the line.

\section{Physical Background}

The mass absorption is often required over a wide range of energies so the usual graphs and tabulations of $\mu / \rho$ are given on a $\mathrm{keV}$ scale. Figure 1a shows the common log-log method of plotting 
IRON K ABSORPTION EDGE-7.11 kEV

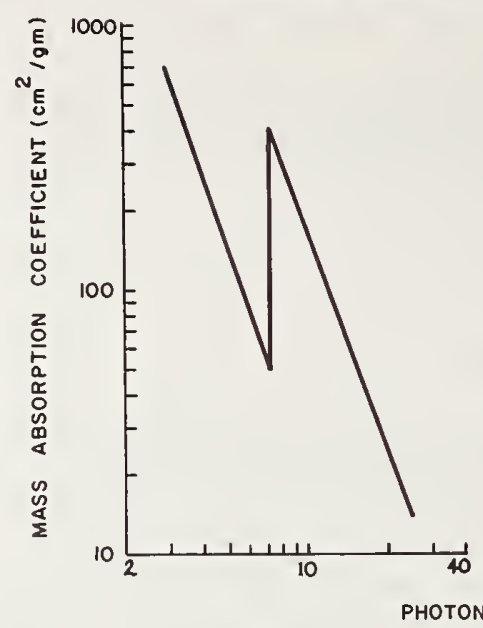

(a)

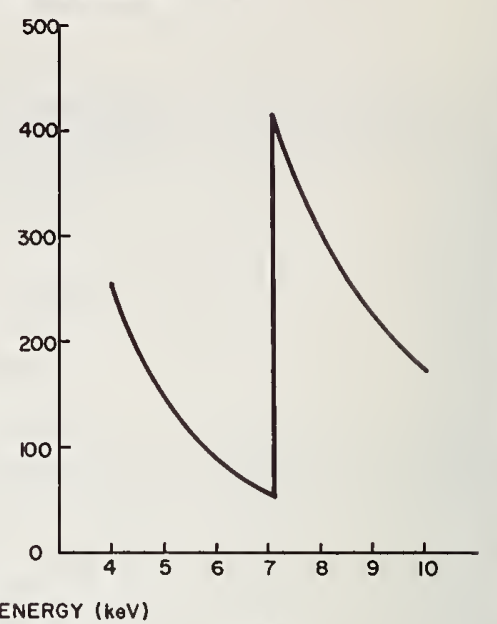

(b)

Figure 1. Conventional graphs of the Iron mass absorption coefficients in the region of the $K$ edge on (a) $\log -\log$ and (b) linear scales [2]. The abscissa is in units of $\mathrm{keV}$.

the mass absorption coefficient which is useful since $\mu / \rho$ varies approximately as $\mathrm{E}^{-3}$. A linear graph of the same region is shown in Figure $1 \mathrm{~b}$. The absorption edge fine structure is contained within a fraction of a $\mathrm{keV}$ of the edge and cannot be plotted well on the scale of Figure 1. An expanded energy scale, generally in electron volts, is necessary to plot the absorption edge fine structure. Figure 2, for example, shows the Iron $\mathrm{K}$ edge measurements of White [3] for Iron in the pure metal and $\mathrm{Fe}_{2} \mathrm{O}_{3}$.

The region above an absorption edge (in energy) can be divided into two physically distinct parts. In the first 30 electron volts (eV) or so above the edge, the fine structure, called Kossel structure. is due to electron transitions to higher unfilled states when a photon is absorbed. These transitions are between bound atomic states. Beyond about $30 \mathrm{eV}$, the Kronig fine structure may persist to as much as $400 \mathrm{eV}$ above the edge. This is the case only for absorbers with high lattice symmetry. In more complex and in amorphous materials the fine structure extends to only about $100 \mathrm{eV}$ above the edge [6]. The Kronig structure is due to the effect of other atoms in the solid on the electron ejected from the atom absorbing the $\mathrm{x}$-ray 


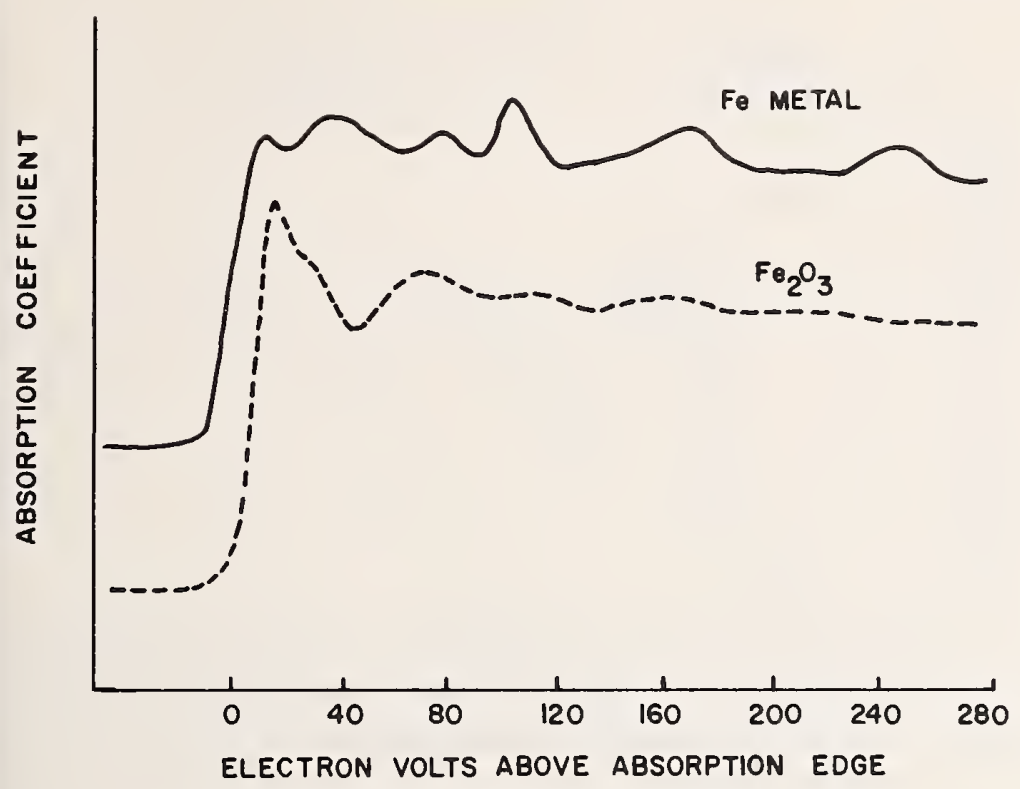

Figure 2. Linear graph of the Iron $\mathrm{K}$ absorption edge fine structure versus electron volts relative to the $\mathrm{K}$ edge at $7.111 \mathrm{keV} \mathrm{[3].} \mathrm{The} \mathrm{curve} \mathrm{for} \mathrm{the} \mathrm{metallic} \mathrm{absorber}$ is displaced vertically from that of $\mathrm{Fe}_{2} \mathrm{O}_{3}$ for clarity. Note the shift in the edge position, and the difference in shape and magnitude of the fine structure for the metal and oxide.

photon. Greatest variations in the mass absorption coefficient occur in the Kossel region where the $\mu / \rho$ value can vary from less than $50 \%$ to more than $150 \%$ of the tabulated value. In the Kronig region the variations are typically about $\pm 5-10 \%$. Figure 2 illustrates how both the Kossel and Kronig structures can vary in shape and magnitude, depending on the chemical environment in which the absorbing element finds itself. It is significant also that the position of the edge varies several $\mathrm{eV}$ from one compound to another.

Figure 3 is a schematic showing the extremes of the fine structure in some absorption edges. The dashed line indicates the value which would usually be tabulated or plotted. Superimposed on the edge structure are four bars indicating emission lines at positions where $\mu / \rho$ would vary appreciably from the tabulated value. These might be lines used to make a measurement in a sample containing the element whose absorption edge is shown. 


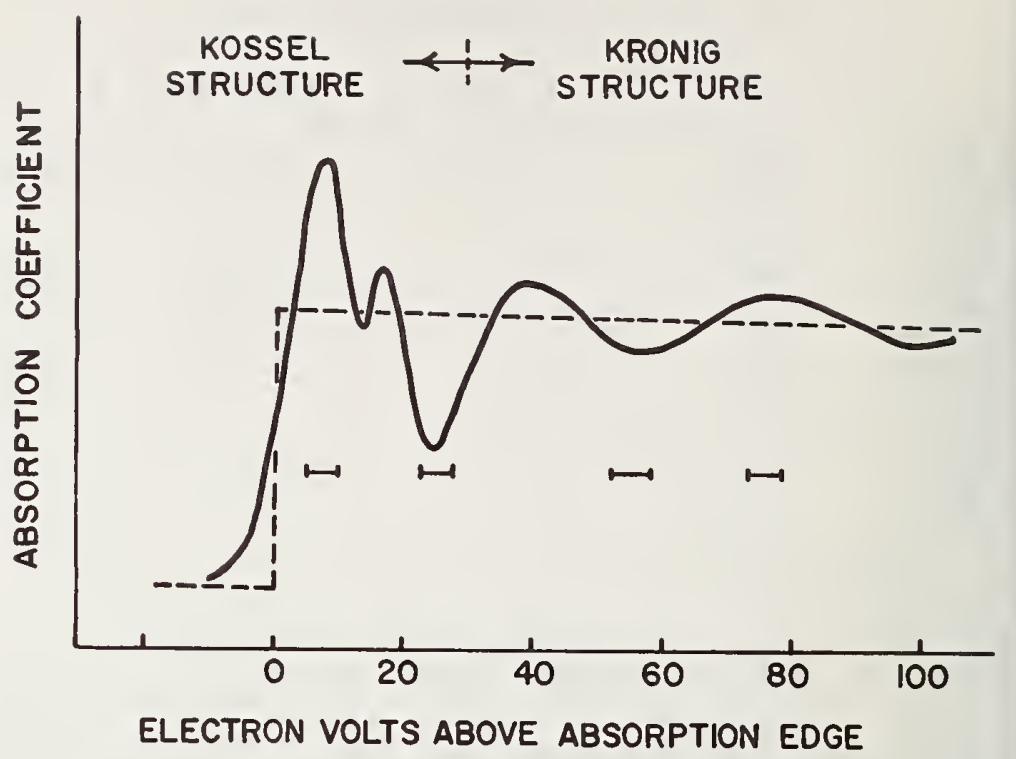

Figure 3. Schematic of an absorption edge fine structure (solid line). The value of the mass absorption coefficient usually tabulated is shown by the dashed line. The bars give the widths of typical emission lines at positions where the actual and tabulated absorption coefficients differ significantly.

The widths of $\mathrm{x}$-ray lines commonly used in microprobe analysis vary from about 2 to $12 \mathrm{eV}$. See Table 1 for examples. Since the period of undulations in the mass absorption coefficient is typically 15 to 20 or more $\mathrm{eV}$, the lines are usually not sufficiently wide to average over the variations.

\section{Effects of Line-Edge Interference}

Examination of wavelength and absorption edge tables [7] shows that intense emission lines which might be used for probe analysis ( $\mathrm{K}$ and $\mathrm{L} \alpha$ and $\beta$ lines, 0.6 to 15 angstroms) frequently fall within $100 \mathrm{eV}$ of an absorption edge. Some of these pairs of interfering elements are mutually insoluble, form no compounds, or are unlikely to occur in microprobe samples. There remain, however, practical samples where emission lines overlap the absorption edge fine structure. It should also be noted that there are many instances when emission lines fall between the various $\mathrm{L}$ or $\mathrm{M}$ edges. In those regions even the smoothed values of $\mu / \rho$ are less well known. 
Table 1. X-ray line widths in electron volts

$\mathrm{K}$ lines [4]

Z

$\mathrm{Ca}(20)$

$a_{1}$

$\alpha_{2}$

1.69

$\mathrm{Cu}(29)$

3.0

6.6

11.1

11.3
L lines [5]

Z

$\operatorname{Ag}(47)$

W (74)

$\mathrm{Pb}(82)$

9.5

8.4

$\mathrm{U}(92) \quad 13.1$

14.3

Test calculations have been made for binary systems to illustrate the effect of uncertainty in $\mu / \rho$, due to the presence of edges, on the absorption and characteristic fluorescence corrections. Analytical conditions and the composition were held constant, and corrections calculated at $0.5,1.0$, and 1.5 of the tabulated $\mu / \rho$ value [1]. Philibert's expression for $f(\chi)$, as modified by Duncumb and Shields, was used for the absorption correction. The fluorescence correction was calculated using the formula of Criss [8] which contains Reed's expression for the effect of voltage on secondary fluorescence. The results are given in Table 2 .

These calculations show that for the $50-50$ or stoichiometric compositions used, the uncertainties in the intensity ratios $\mathrm{k}_{\mathrm{a}}$ and $\mathrm{k}_{\mathrm{f}}$ are usually $1-3 \%$. However, in the $50 \mathrm{Mg}-50 \mathrm{Ge}$ system, a $\pm 50 \%$ error in $\mu / \rho$ produces an error in $\mathrm{k}_{\mathrm{a}}$ of +18.4 or $-7.2 \%$. For the intermetallic compound $\mathrm{Hf}_{2} \mathrm{Cu}$, the error in $\mathrm{k}_{\mathrm{f}}$ is about $7 \%$ due to the $50 \% \mu / \rho$ error. The errors in $\mathrm{k}_{\mathrm{a}}$ or $\mathrm{k}_{\mathrm{f}}$ would be worse for compositions nearer the end members. The analysis for $\mathrm{Cu}$ in $\mathrm{Hf}_{2} \mathrm{Cu}$ provides an example of this. While errors in corrections for systems near end members yield small absolute errors in the calculated concentrations, the relative errors would be large. The above examples are for errors appropriate to the Kossel structure. Errors due to Kronig structure are considerably smaller.

\section{Discussion}

It is seen that uncertainty in the $\mu / \rho$ value near absorption edges can lead to errors in the absorption and fluorescence corrections which are unacceptably large in some cases. These errors can be 
Table 2. Effect of absorption coefficient variation on absorption and fluorescence corrections

(20 keV, $30^{\circ}$ takeoff angle)

\begin{tabular}{|c|c|c|c|c|c|c|c|c|}
\hline $\mathrm{Z}_{\mathrm{I}}$ & $\mathrm{Z}_{2}$ & Wt.\% $\mathrm{Z}_{1}$ & Lines & Edge & $\Delta(\mathrm{eV})^{\mathrm{a}}$ & $\mu / \rho$ ratio $^{b}$ & $\left(k_{a}\right) z_{1}^{c}$ & $\left(k_{f}\right) z_{2}^{c}$ \\
\hline \multirow[t]{3}{*}{$\mathrm{Br}$} & $\mathrm{Au}$ & 28.8 & $\mathrm{BrKa} a_{1}$ & AuLIII & 3 & 0.5 & 0.990 & 1.020 \\
\hline & & $(\mathrm{AuBr})$ & $A u L a_{1}$ & & & $1: 0$ & 0.975 & 1.029 \\
\hline & & & & . & & 1.5 & 0.959 & 1.032 \\
\hline \multirow[t]{3}{*}{ Hf } & $\mathrm{Cu}$ & 15.1 & HfL $\beta_{1}$ & $\mathrm{CuK}$ & 24 & 0.5 & & 1.099 \\
\hline & & $\left(\mathrm{Hf}_{2} \mathrm{Cu}\right)$ & $\mathrm{CuKa}_{1}$ & & & 1.0 & $d$ & 1.178 \\
\hline & & & & & & 1.5 & & 1.248 \\
\hline \multirow[t]{3}{*}{$\mathrm{Mg}$} & $\mathrm{Ge}$ & 50 & $\mathrm{MgK} \alpha$ & GeLIII & 36 & 0.5 & 0.383 & \\
\hline & & & GeLa 1 & & & 1.0 & 0.199 & d \\
\hline & & & & & & 1.5 & 0.127 & \\
\hline \multirow[t]{3}{*}{$\mathrm{Au}$} & $\mathrm{Zn}$ & 50 & $A_{u L} a_{1}$ & $\mathrm{ZnK}$ & 52 & 0.5 & 0.999 & 1.034 \\
\hline & & & $\mathrm{ZnK}_{\alpha_{1}}$ & & & 1.0 & 0.980 & 1.053 \\
\hline & & & & & & 1.5 & 0.960 & 1.066 \\
\hline \multirow[t]{3}{*}{ W } & $\mathrm{Ni}$ & 50 & WLa 1 & $\mathrm{NiK}$ & 65 & 0.5 & 0.997 & 1.032 \\
\hline & & & $\mathrm{NiK}_{1}$ & & & 1.0 & 0.960 & 1.049 \\
\hline & & & & & & 1.5 & 0.936 & 1.061 \\
\hline
\end{tabular}

\section{Notes:}

a $\Delta(\mathrm{eV})=$ difference between tabulated line and edge energies in electron volts $[7]$ b $\mu / \rho$ ratio $=(\mu / \rho)$ actual $/(\mu / \rho)$ tabulated.

${ }^{\mathrm{c}}$ Concentration $=($ Relative $X$-ray Intensity $) \times\left(\mathrm{k}_{\text {absorption }}\right) \times\left(\mathrm{k}_{\text {fluorescence }}\right)=\mathrm{rk}_{\mathrm{a}} \mathrm{k}_{\mathrm{f}}$.

"Other lines would probably be used for an analysis.

outside the expected accuracy of measurement and calculation. The existence and source of such an error in the answer for one element whose line overlaps an edge fine structure might not be obvious. Normalization of the total composition to $100 \%$ would cause the error in one element to have an effect on the analysis for all the elements in the system.

Instances of interference between a measured line and an edge are relatively infrequent in binary probe samples, but become more likely in ternary and more complicated systems. The existence of 
such interferences can be checked most easily using Table 2 of Bearden [7] where all x-ray lines and edges are listed in order of energy. The region within about $400 \mathrm{eV}$ below each line intended to be used in an analysis can be examined for the presence of the edge of any of the other elements in the sample. Other tables of x-ray lines and edges can be used also.

If an interference is found, another line should be used if possible. When this cannot be done, it must be expected that $\mu / \rho$ might be in error by $\pm 50 \%$ within about $30 \mathrm{eV}$ of the edge or by $\pm 5-10 \%$ within 30 to about $400 \mathrm{eV}$ above the edge. Since accurate absorption edge fine structure measurements are not available for all edges, and since the position of the edge and the shape of the fine structure depend on the chemistry of the sample, it is not possible to determine the difference between actual and tabulated $\mu / \rho$ values (except by measurement of $\mu / \rho$ ). Calculation of the absorption and fluorescence corrections using $\mu / \rho$ values near the extremes of the range of variation (which depends on the line position above the edge) would show the sensitivity of the results to the mass absorption coefficient, or, at least, indicate the size of the possible error. When the $\mu / \rho$ uncertainty adversely affects one of the corrections, changing the takeoff angle may alleviate the problem, if this is possible.

Measurements of broad soft x-ray lines, using a proportional or scintillation counter to include the entire line, would tend to average over $\mu / \rho$ variations near edges. Use of broad lines is not necessarily advantageous, however, since microprobe correction procedures are implicity derived for the usual narrow x-ray lines.

The problem of line-edge interferences, discussed above for the electron probe, exists similarly for x-ray fluorescence analysis. It can be expected to occur more often in the latter case since fluorescence analysis samples can be physical mixtures, e.g., powders, and often contain many elements.

\section{Acknowledgments}

This problem was pointed out by Dr. R. Deslattes of the National Bureau of Standards. The corrections summarized in Table 2 were calculated by J. W. Criss. The author gratefully acknowledges this assistance and the critical reading of the manuscript by L. S. Birks. 


\section{References}

[1] Heinrich, K. F. J., "X-Ray Absorption Uncertainty", The Electron Microprobe. John Wiley \& Sons, New York, 1966.

[2] McMaster, W. H., et al., X-Ray Cross Sections, UCLRL Report 50174 Part II, January 1967.

[3] White, E. W., "Chemical Effects on X-Ray Absorption Edge Fine Structure", Advances in X-Ray Analysis, Vol. 9, Plenum Press, New York, 1966.

[4] Compton, A. H., and Allison, S. K., X-Rays in Theory and Experiment, 2nd edition, Van Nostrand, Princeton, 1935.

[5] Blokhin, M. A., The Physics of X-Rays, State Publishing House of TechnicalTheoretical Literature, Moscow, 1957 and AEC-tr-J502, 1961.

[6] Van Nostrand, R. A., "Fine Structure in Absorption Edge Spectra", X-Ray Handbook, McGraw Hill Book Co., New York, 1967.

[7] Bearden, J. A., X-Ray Wavelengths, Atomic Energy Commission, NYO 10586 , Oak Ridge, Tennessee, 1964.

[8] Criss, J.W., "Progress in Quantitative Electron Probe Microanalysis", Fifty" Years of Progress in Metallographic Techniques, ASTM STP 430, Am. Soc? Testing Materials, 1967. 


\title{
FLUORESCENCE EXCITED BY THE CONTINUUM \\ J. HÉNOC
}

National Center for Studies of Telecommunication,

Issy les Moulineaux, France

\begin{abstract}
The intensity of secondary radiation excited by the continuous spectrum is calculated in a form suitable for computer evaluation of the numerical values. The parameter of interest is the ratio of secondary emission to the total emerging intensity of radiation; this ratio $\left(\mathrm{I}_{(A)} / \mathrm{I}_{(A)}\right)$ has been calculated for several elements choosing an appropriate expression for primary radiation. The direct measurement of the ratio $\left(\mathrm{I}_{(A)} / \mathbf{I}_{(A)}\right)$ and of $\mathrm{I}_{(A)}$, performed on the same elements, provides a test of the correctness of the calculations and a basis for a proposed semi-empirical method for routine evaluation of the corrections.
\end{abstract}

\section{Introduction}

A target being subjected to the impact of a beam of suitably accelerated electrons emits $X$ radiation in the characteristic lines of the atoms present in this target. The characteristic radiation is due to the ionization of the inner shells of the atom by electrons of the beam and by the absorption of the continuum emitted during the event of electron energy loss in the material. Under normal microanalytical test conditions, the first mentioned phenomenon prevails, though it is no longer allowable to neglect the second one, in view of the considerable advances achieved, especially in the field of spectrometer sensitivity.

We are going to set forth a method for calculating the intensity of fluorescence radiation excited by the continuum in order to evaluate the correction for it in the results of electron probe microanalysis. In the first (theoretical) part we shall derive the formula for the intensity of the fluorescence radiation and decide upon a method for calculating the primary radiation directly excited by electrons in order to obtain the all inclusive ratio between intensities of fluorescence radiation and primary radiation. In the second, more practical, part, we shall report on a few tests made, in order to 
render the application of calculations more explicit, and indicate a way of effecting corrections for fluorescence excited by the continuum in routine analyses.

\section{Theory}

\section{A. CALCULATION OF FLUORESCENCE EMISSION}

The evaluation of the fluorescence èmission excited by the continuum is possible on the basis of two simplifying assumptions. First, it is assumed that the continuous spectrum is emitted at a depth which is negligible compared with its depth of penetration into the sample; this amounts to assuming that the primary radiation source is a pin-point source on the surface of the specimen. In a general way, it is worthwhile noting that the distribution form of the primary radiation as a function of depth interferes but little in the calculation of fluorescence and that the intensity of the fluorescence radiation is dependent only on the total intensity of the primary radiation. It will also be assumed that the continuous radiation is isotropic.

The intensity of fluorescence radiation emerging from the specimen due to excitation by the spectral component of the continuum whose wavelength and intensity are $\lambda$ and $\mathbf{I}_{\lambda}{ }^{c}$, respectively, with reference to Figure 1 , is given by the following expression:

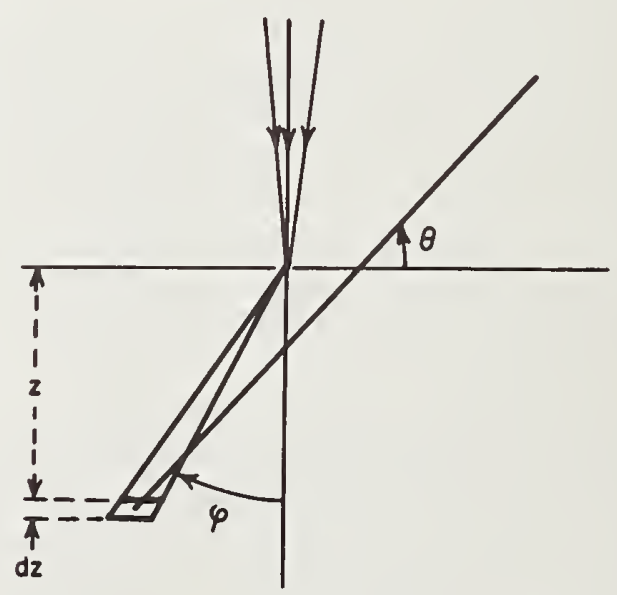

Figure 1. Emission of secondary radiation. 


$$
\mathrm{I}_{A, \lambda}^{\mathrm{f}}=\frac{\Omega}{4 \pi} \frac{1}{2} \mathrm{C}_{A} \mu_{\lambda}^{\mathrm{A}} \omega_{1}(\mathrm{~A}) \frac{\mathrm{r}_{j}-1}{\mathrm{r}_{k}} \mathrm{Z}_{1}(\mathrm{~A}) \mathrm{I}_{\lambda}^{\mathrm{C}} \times
$$

$\times \int_{0}^{\frac{\pi}{2}} \mathrm{~d} \phi \int_{0}^{\infty} \operatorname{tg} \phi \exp \left\{-\rho \mathrm{z}\left(\frac{\mu_{\lambda}^{\mathrm{AB}}}{\cos \phi}+\frac{\mu_{A}^{\mathrm{AB}}}{\sin \theta}\right)\right\} \mathrm{d}(\rho \mathrm{z})$

$$
\begin{aligned}
& \mathrm{I}_{A, \lambda}^{\mathrm{f}}= \frac{\Omega}{4 \pi} \frac{1}{2} \frac{\mathrm{C}_{A} \mu_{\lambda}^{\mathrm{A}}}{\mu_{\lambda}^{\mathrm{AB}}} \omega_{1}(\mathrm{~A}) \frac{\mathrm{r}_{j}-1}{\mathrm{r}_{k}} \mathrm{Z}_{1}(\mathrm{~A}) \mathrm{I}_{\lambda}^{\mathrm{c}} \times \\
& \times \frac{\mathrm{L}\left[1+\frac{\mu_{A}^{\mathrm{AB}} \operatorname{cosec} \theta}{\mu_{\lambda}^{\mathrm{AB}}}\right]}{\frac{\mu_{A}^{\mathrm{AB}} \operatorname{cosec} \theta}{\mu_{\lambda}^{\mathrm{AB}}}}
\end{aligned}
$$

The intensity of the total fluorescence radiation is obtained by finding the summation of this quantity over the range extending from the minimum wavelength $\lambda_{o}$ to a wavelength $\lambda_{i}$ of the level producing the characteristic line chosen for the analysis, using as many intervals as there exist absorption edges between $\lambda_{o}$ and $\lambda_{i}$. The expression giving the intensity of the continuous radiation as a function of wavelength, $I \ell=K Z\left(1 / \lambda_{0}-1 / \lambda\right) 1 / \lambda$ is due to Kramers (1923) [1]; it is in good agreement with the empirical formula proposed by Kulenkampff (1924) [2]. Green (1962) [3] has shown that the constant $\mathrm{K}$ whose value was initially assumed equal to 2.26 $x 10^{-13} \mathrm{~cm}$, varies with the atomic number. In case of a compound specimen, we assume that we can write $\mathrm{Z}=\mathrm{C}_{A} \mathrm{Z}_{A}+\mathrm{C}_{B} \mathrm{Z}_{B}$. The absorption coefficient variation law versus wavelength allows us to

${ }^{1}$ Note: list of symbols at end of the paper 
write $\mu_{\lambda}^{x}=\mu_{\lambda \theta}^{x}\left(\lambda / \lambda_{0}\right)^{3}$, provided that there is no absorption edge within the interval $\left(\lambda_{0}, \lambda_{i}\right)$. In the case of interval $\left(\lambda_{0}, \lambda_{i}\right)$ containing an absorption edge $i$, this expression is to be divided by the jump $r_{i}$ relative to the absorption edge at the instant of passing the latter. We shall then write: $\mu_{\lambda}^{x}=\mu_{\lambda}^{x}=\left(\mu_{\lambda_{0}}^{x} / r_{i}\right)\left(\lambda / \lambda_{0}\right)^{3}$.

Substituting in (1) for $I_{\lambda}, \mu_{\lambda}^{A}$ and $\mu_{\lambda}^{A B}$ results in:

$$
\mathrm{I}_{A}^{\mathrm{f}}=\sum_{\mathrm{m}=0}^{l-1} \int_{\lambda_{\mathrm{m}}}^{\lambda_{m+1}} \mathrm{I}_{A, \lambda}^{\mathrm{f}} \mathrm{d} \lambda=\sum_{\mathrm{m}} \frac{\Omega}{4 \pi} \frac{1}{2} \frac{\mathrm{C}_{A} \mu \dot{\lambda}_{o}^{\mathrm{A}}}{\mu \lambda_{o}^{\mathrm{AB}}} \omega_{1}(\mathrm{~A}) \frac{\mathrm{r}_{j}-1}{\mathrm{r}_{k}}
$$

$$
\cdot \mathrm{Z}_{I}(\mathrm{~A}) \mathrm{KZ} \int_{\lambda_{m}}^{\lambda_{m+1}}\left(\frac{1}{\lambda_{o}}-\frac{1}{\lambda}\right) \frac{1}{\lambda} \cdot \frac{\left[1+\frac{\mu_{A}^{\mathrm{AB}} \operatorname{cosec} \theta}{\mu_{\lambda_{\circ}^{\mathrm{AB}}}\left(\frac{\lambda}{\lambda_{o}}\right)^{3}}\right]}{\frac{\mu_{A}^{\mathrm{AB}} \operatorname{cosec} \theta}{\mu_{\lambda_{o}^{\mathrm{A} \mathrm{B}}}^{\mathrm{B}}\left(\frac{\lambda}{\lambda_{o}}\right)^{3}}} \mathrm{~d} \lambda
$$$$
\mathrm{I}_{A}^{\mathrm{f}}=\sum_{\mathrm{m}} \mathrm{E}_{A B}^{\mathrm{m}}\left\{\Phi\left(\lambda_{o}, \lambda_{m+1}, \mathrm{v}_{A B}^{\mathrm{m}}\right)-\phi\left(\lambda_{\circ}, \lambda_{m}, \mathrm{v}_{A B}^{\mathrm{m}}\right)\right\}
$$

$$
\mathrm{E}_{A B}^{\mathrm{m} B}=\frac{\Omega}{4 \pi} \frac{1}{2} \frac{\mathrm{C}_{A} \mu_{\lambda_{0}}^{\mathrm{A}}}{\mu_{\lambda_{0}}^{\cdot \mathrm{AB}}} \quad \omega_{1}(\mathrm{~A}) \frac{\mathrm{r}_{j}-1}{\mathrm{r}_{k}} \mathrm{Z}_{1}(\mathrm{~A}) \mathrm{KZ}
$$




$$
\Phi\left(\lambda_{0}, \lambda, \mathrm{v}\right)=\frac{1}{\lambda_{\mathrm{o}} \mathrm{v}^{3}}\left\{\left[\frac{1}{3}\left(\frac{\lambda}{\lambda_{0}}\right)^{3}-\frac{1}{2}\left(\frac{\lambda}{\lambda_{0}}\right)^{2}\right] \mathrm{L}\left[1+\left(\frac{\lambda_{o} \mathrm{v}}{\lambda}\right)\right]+\right.
$$$$
\left(\frac{\mathrm{v}^{3}}{3}+\frac{\mathrm{v}^{2}}{2}\right) \mathrm{L}\left(\lambda+\lambda_{o} \mathrm{v}\right)+\left(\frac{\mathrm{v}^{3}}{3}-\frac{\mathrm{v}^{2}}{4}\right) \mathrm{L}\left(\lambda^{2}-\lambda_{o} \lambda \mathrm{v}+\lambda_{\circ}^{2} \mathrm{v}^{2}\right)-
$$

$$
\left.\left(\frac{\sqrt{3}}{2}\right) v^{2} \operatorname{arctg} \frac{\frac{\lambda}{\lambda_{o}}-\frac{v}{2}}{\frac{\sqrt{3}}{2} v}\right\}
$$

$$
\left(\mathrm{v}_{A B B}^{\mathrm{m}}\right)^{3}=\frac{\mu_{A}^{\mathrm{AB}} \operatorname{cosec} \theta}{\mu_{\lambda_{0}}^{\mathrm{AB}}}
$$

Formula (4) giving the quantity $\phi\left(\lambda_{o}, \lambda, v\right)$ can be tabulated by means of a computer in the following way:

$\lambda_{o}$ is chosen among the values corresponding to the operating voltage $(5-45 \mathrm{kV}$, for instance, staggered $5 \mathrm{kV}$ apart). For this value of $\lambda_{o}, \lambda_{i}$ is taken among $\mathrm{K}$ or $\mathrm{L}$ absorption edges of the various elements. The value of $\phi$ is then read from the table, for $\mathrm{v}^{3}$ chosen in the range $(0,100)$, staggering: 0.5 . The $\phi$ function involving only wavelengths and parameter $\mathrm{v}^{3}$ can be tabulated once and for all. Figure 2 is a representation thereof.

It has been assumed that the wavelength variation interval contains no edge from element B. If this were not the case it would be sufficient to add merely one extra interval being limited by the absorption edge of $\mathrm{B}$, by introducing into $\mu_{\lambda a}^{\prime A B}$ the edge jump ratio relative to this edge. As an indication, Table I provides the values of the different parameters in each wavelength variation interval.

\section{B. CALCULATION OF THE PRIMARY RADIATION}

The absolute calculation of the fluorescence radiation alone has only a theoretical interest, when one wishes to know its contribution to the total emerging radiation, as in the case of microanalysis. 


$$
\lambda_{\mathrm{o}}
$$

$$
\lambda
$$

$$
\mathrm{V}^{3}
$$

$\Phi$

$\Delta / 5 \times 10^{4}$

$\begin{array}{lllll}0.413 & 2.926 & 60.50 & 2.9677 & 5.5630 \\ 0.413 & 2.926 & 61.00 & 2.9704 & 5.5112 \\ 0.413 & 2.926 & 61.50 & 2.9731 & 5.4607 \\ 0.413 & 2.926 & 62.00 & 2.9759 & 5.4108 \\ 0.413 & 2.926 & 62.50 & 2.9785 & 5.3619 \\ 0.413 & 2.926 & 63.00 & 2.9812 & 5.3140 \\ 0.413 & 2.926 & 63.50 & 2.9838 & 5.2670 \\ 0.413 & 2.926 & 64.00 & 2.9864 & 5.2208 \\ 0.413 & 2.926 & 64.50 & 2.9890 & 5.1752 \\ 0.413 & 2.926 & 65.00 & 2.9916 & 5.1307 \\ 0.413 & 2.926 & 65.50 & 2.9941 & 5.0870 \\ 0.413 & 2.926 & 66.00 & 2.9967 & 5.0437 \\ 0.413 & 2.926 & 66.50 & 2.9992 & 5.0016 \\ 0.413 & 2.926 & 67.00 & 3.0016 & 4.9600 \\ 0.413 & 2.926 & 67.50 & 3.0041 & 4.9191 \\ 0.413 & 2.926 & 68.00 & 3.0065 & 4.8788 \\ 0.413 & 2.926 & 68.50 & 3.0090 & 4.8395 \\ 0.413 & 2.926 & 69.00 & 3.0114 & 4.8005 \\ 0.413 & 2.926 & 69.50 & 3.0137 & 4.7624 \\ 0.413 & 2.926 & 70.00 & 3.0161 & 4.7249 \\ 0.413 & 2.926 & 70.50 & 3.0184 & 4.6877 \\ 0.413 & 2.926 & 71.00 & 3.0208 & 4.6515 \\ 0.413 & 2.926 & 71.50 & 3.0231 & 4.6156 \\ 0.413 & 2.926 & 72.00 & 3.0254 & 4.5807 \\ 0.413 & 2.926 & 72.50 & 3.0276 & 4.5457\end{array}$

Figure 2. Calculation of $\Phi$. Section of computer output. 
TABLE I

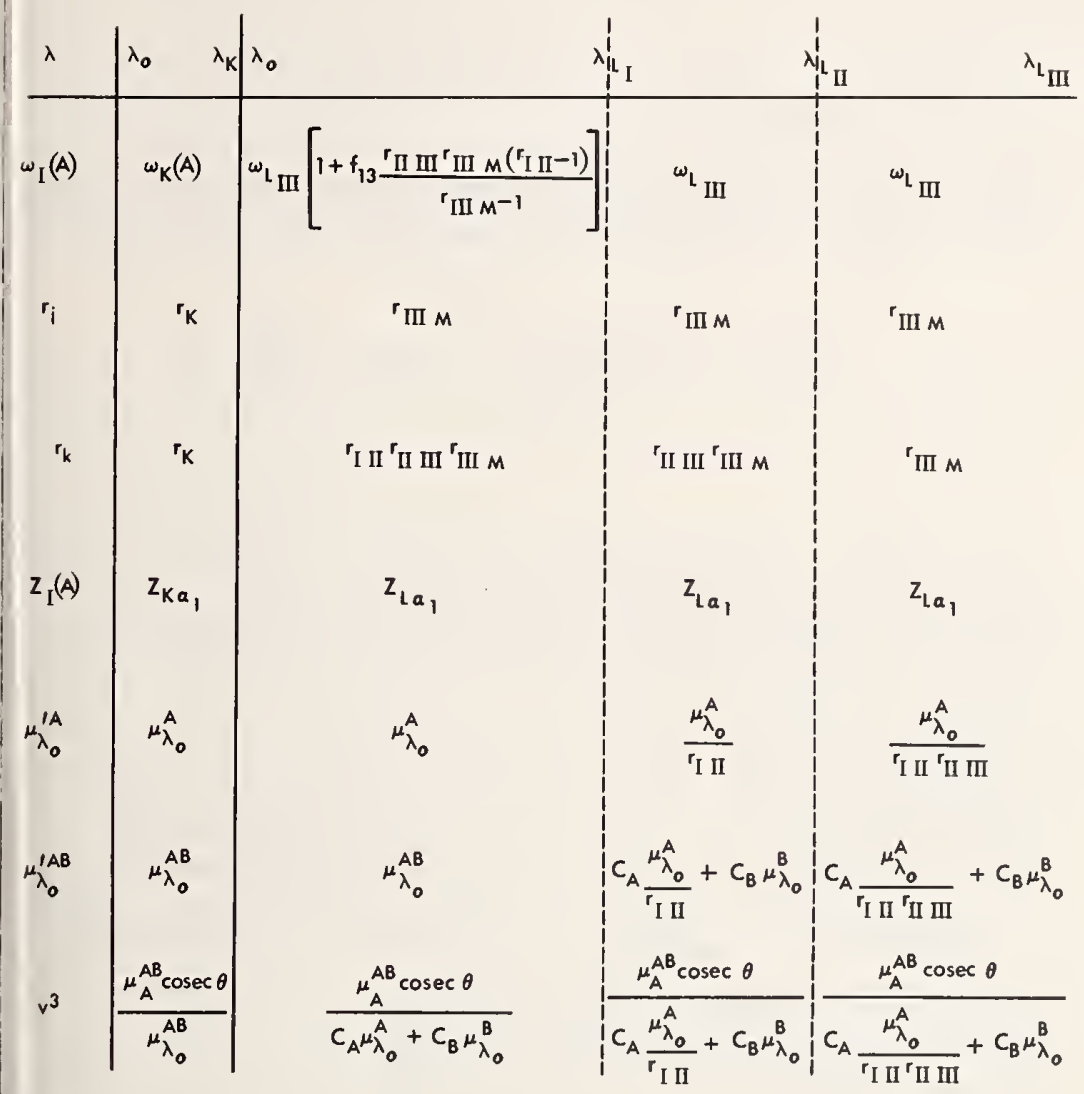

Therefore it is essential to evaluate it relative to the intensity of the primary radiation. Assuming that the proportionality law between emission and concentration holds for the primary radiation, one can restrict oneself to the case of a particular element. Let $Q\left(E, E_{l}\right)$ be the ionization cross-section; the number of ionizations, $\mathrm{dn}_{A}$, produced by an electron having an energy $E$ and travelling over a path element $\mathrm{dx}$ within a target made of element $\mathrm{A}$ is given by the expression:

$$
\mathrm{dn}_{A}=\frac{\mathrm{N} \rho_{A}}{\mathrm{~A}} \mathrm{Q}\left(\mathrm{E}, \mathrm{E}_{1}\right) \mathrm{dx}
$$


The corresponding number of photons emitted in the L-line is

$$
\mathrm{dI}_{(A)}^{o}=\omega_{1} \text { (A) } \mathrm{Z}_{1} \text { (A) } \frac{\mathrm{N} \rho_{A}}{\mathrm{~A}} \mathrm{Q}\left(\mathrm{E}, \mathrm{E}_{\ell}\right) \frac{\mathrm{dx}}{\mathrm{dE}} \mathrm{dE}
$$

Over the path along which the energy decreases from $E_{o}$ to $E_{l}$, the electron produces a total number of photons given by the expression

$$
\mathrm{I}_{(A)}^{\circ}=\omega_{1}(\mathrm{~A}) \mathrm{Z}_{1} \text { (A) } \frac{\mathrm{N} \rho_{A}}{\mathrm{~A}} \int_{\mathrm{E}_{o}}^{\mathrm{E}_{1}} \mathrm{Q}\left(\mathrm{E}, \mathrm{E}_{1}\right) \frac{\mathrm{dx}}{\mathrm{dE}} \mathrm{dE}
$$

The quantity $I_{(A)}^{\circ}$ can be calculated if the ionization function $\mathrm{Q}\left(\mathrm{E}, \mathrm{E}_{1}\right)$ and the expression for the electron energy-loss $\mathrm{dE} / \mathrm{dx}$ are known. One can use both formulas derived by Bethe as quoted by Mott and Massey (1949) [4].

$\mathrm{Q}=\frac{\pi \mathrm{e}^{4}}{\mathrm{EE}_{1}} \mathrm{Z}_{n 1}$ bL $\frac{4 \mathrm{E}}{\mathrm{B}}$

$$
\text { where } \quad \begin{aligned}
b & =0.35 \text { for } K \text {-shells } \\
b & =0.25 \text { for L-shells } \\
B & =1.65 \mathrm{E}_{K}\left(\text { or } \mathrm{E}_{e}\right)
\end{aligned}
$$

$\mathrm{Z}_{n l}=$ No. of electrons of shell.

$-\frac{\mathrm{dE}}{\mathrm{dx}}=\frac{2 \pi \mathrm{e}^{4} \mathrm{~N} \rho_{A} \mathrm{Z}_{A}}{\mathrm{AE}} \quad \mathrm{L} \frac{2 \mathrm{E}}{\mathrm{J}}$

where

$\mathbf{J}=$ mean ionization potential

Substituting (7) and (8) in (4) results in:

$$
\begin{aligned}
\mathrm{I}_{(A)=\omega_{I}}^{\circ}(\mathrm{A}) \mathrm{Z}_{I}(\mathrm{~A}) \frac{\mathrm{bZ} \mathrm{Z}_{n 1}}{2 \mathrm{E}_{1} \mathrm{Z}_{A}}\left\{\frac{\mathrm{J}}{2}\left[\mathrm{Ei}\left(\mathrm{L} \frac{2 \mathrm{E}_{o}}{\mathrm{~J}}\right)-\mathrm{Ei}\left(\mathrm{L} \frac{2 \mathrm{E}_{l}}{\mathrm{~J}}\right)\right] \mathrm{L} \frac{2 \mathrm{~J}}{\mathrm{~B}}+\right. \\
\left.+\mathrm{E}_{o}-\mathrm{E}_{1}\right\}
\end{aligned}
$$


More recently, a rule-of-thumb formula has been proposed by Green (1962) [3]. The ionization cross-section adopted by Green is derived from that established by Bethe in which the different coefficients were modified according to indications by Worthington and Tomlin (1956) [5]. The expression which has been chosen for the electron energy-loss, is the Thomson-Whiddington law (1912) [6]. Green thus obtains the number of photons emitted in the $\mathrm{K}$ spectrum in the following form:

$$
\frac{\mathrm{I}_{K}}{4 \pi}=\omega_{K} \cdot 2.8 \times 10^{3} \frac{\mathrm{R}}{\mathrm{Ac}}\left(\frac{\mathrm{E}_{o}}{\mathrm{E}_{K}}-1\right)^{1.67}
$$

where $\mathbf{R}$ is the back-scattering coefficient and $\mathrm{c}$ the ThomsonWhiddington constant.

\section{CALCULATION OF $\mathbf{I}_{(A)} / \mathbf{I}_{(A)}$}

Expressions (2) and (9) for instance, permit the calculation of the relative importance of the fluorescence radiation $\mathbb{I}_{(A)}$ excited by the continuum with respect to the total radiation $\mathbf{I}_{(A)}$ for a particular element. The calculation has been made for the zinc $\mathrm{K} \alpha_{1}$-line, the tungsten, gold, and bismuth $\mathrm{L} \alpha_{1}$-lines, with accelerating voltages of $30 \mathrm{kV}$, using the most recent values for the different physical constants relating to these elements. It is worthwhile noting that a difficulty arises in the calculation of $\mathrm{L} \alpha_{1}$-line intensities due to the fact that values for $\omega_{l}(\mathrm{~A})$ appearing in (2) and (9) are not identical. Strictly speaking, instead of the fluorescence yield $\omega_{L I I} \cdot$ relative to $\mathrm{L}_{I I I}$ transitions, one should use an "effective" yield allowing for Coster-Kronig transition coefficients. The situation is then different whether we are confronted with an ionization event produced by photons or by electrons. It is easy to observe this for $74<\mathrm{Z}<83$, where, according to Listengarten (1960) [7] $\mathrm{f}_{23}=0$, the values for the "effective" fluorescence yield are listed in table I for ionization by photons. Provided that the energy of incident electrons is markedly larger than that of $\mathrm{L}$ subshells, one can reasonably write $\left(\omega_{L I I I}\right)_{e f f .} \simeq \omega_{L I I I}\left[1+1 / 2 \mathrm{f}_{13}\right]$. Results of calculations are provided below for a take-off angle of approximately $16^{\circ}$.

\begin{tabular}{lcccc} 
& $\mathrm{K}_{1}$ & \multicolumn{3}{c}{$\mathrm{L}_{1}$} \\
Element & $\mathrm{Zn}$ & $\mathrm{Au}$ & $\mathrm{Bi}$ \\
$\frac{\mathrm{I}_{(A)}^{\mathrm{f}}}{\mathrm{I}_{(A)}}$ & 0.031 & 0.025 & 0.036 & 0.037
\end{tabular}




\section{Tests and Applications}

Prior to entering into details relating to practical calculations of corrections, we must check the results of the preceding paragraph. This comparison with the actual experiment can be made in two steps. One can for each of the particular elements mentioned previously first measure the total intensity emitted, and then measure directly the ratio $I_{(A)} / I_{(A)}$ by applying the aluminium method. (See III. B below).

\section{A. MEASUREMENT OF THE EMERGING RADIATION INTENSITY}

The emerging radiation intensity of zinc was measured by applying the double-filter method, the voltage being $30 \mathrm{kV}$. Besides the value of the intensity emitted in the characteristic radiation $\mathrm{K} \alpha_{1}$, estimated at $1.73 \times 10^{-3}$ phot/electron, this method permits the evaluation of the $\mathrm{K}$ constant of Kramers' formula; we have found that $\mathrm{K}=2.64 \times 10^{-13} \mathrm{~cm}$. These experimental results are in good agreement with measurements performed by Green (1962) [3]. Measurements made with a spectrometer on tungsten, gold, and bismuth specimens can be rendered absolute by comparing with tests made on zinc. The agreement observed between calculated and measured values is considerably less consistent for $\mathrm{L} \alpha_{1}$-lines. In order to take the whole set of measurements into consideration, one is led to adopt the following results:

\begin{tabular}{lccccc} 
& $\mathrm{K}_{I}$ & \multicolumn{4}{c}{$\mathrm{L}_{1}$} \\
\cline { 4 - 6 } Element & $\mathrm{Zn}$ & $\mathrm{W}$ & $\mathrm{Au}$ & $\mathrm{Bi}$ \\
$\frac{\mathrm{I}(A)}{\mathrm{I}_{(A)}}$ corrected & .031 & .022 & .044 & .048
\end{tabular}

\section{B. ALUMINIUM METHOD}

This experiment consists of studying the characteristic radiation of a stratified specimen which, in fact, is a block of element $\mathrm{A}$ covered by a film of aluminium which is just sufficient for the energy of a traversing electron to be below the excitation threshold of element $\mathrm{A}$. The characteristic radiation of element $\mathrm{A}$ emitted by this specimen is therefore fundamentally the secondary fluorescence radiation excited by the continuous spectrum emitted by aluminium 
(Castaing and Descamps (1955) [8]). The intensity from the stratified specimen, $\mathbf{I}^{\prime} f_{(A)}$ is compared with the intensity $\mathrm{I}_{(A)}$ of the radiation emitted in the same line by a pure standard $A$.

It is easily seen that the desired result is given by the formula:

$$
\frac{\mathrm{I}_{(A)}^{\mathrm{f}}}{\mathrm{I}_{(A)}}=\frac{\mathrm{Z}_{A}}{13} \frac{1}{\mathrm{f}_{1}} \frac{\exp \left\{-\mu_{A}^{A} \rho_{A}\left[\chi+\mathrm{k}\left(\mathrm{e}_{o}-\epsilon\right)\right] \operatorname{cosec} \theta\right\}}{\exp \left\{-\mu_{A}^{A 1} \rho_{A I} \mathrm{I}_{(A)} \mathrm{e}_{\circ} \operatorname{cosec} \theta\right\}}(
$$

where:

$X=$ thickness of a layer of element $A$ corresponding to a mean emitting depth for primary radiation;

$\epsilon=$ thickness of an aluminium layer corresponding to the mean emitting depth for primary radiation, in aluminium;

$\mathrm{k}=$ ratio between linear absorption coefficients of aluminium and element $\mathrm{A}$ for the continuous spectrum;

$\mathrm{e}_{o}=$ thickness of the aluminium layer

The corrective term which is most difficult to evaluate is $1 / f_{1}$ (Kirianenko et al., 1964) [9]. It represents the proportion of the continuous radiation being absorbed by the aluminium layer of thickness $\left(\mathrm{e}_{0}-\epsilon\right)$. It can be estimated by default by defining a mean absorption coefficient; or, more simply, one can determine an upper limit by comparing the intensity of the primary radiation which is absorbed in the layer with the total intensity of the continuous radiation, assuming that the fluorescence radiation absorption in the layer is negligible. The desired value is then easily derived from the total intensity of the radiation emitted before absorption. As an example, let us quote the case of zinc for a voltage of $30 \mathrm{kV}$; the test is performed by evaporating a $2 \mathrm{mg} / \mathrm{cm}^{2}$ aluminium layer on a polished zinc block. It is assumed that the emission takes place at a mean depth which corresponds approximately to $0.5 \mathrm{mg} / \mathrm{cm}^{2}$. Before exciting the fluorescence radiation from zinc, the continuum is thus partially absorbed by a $1.5 \mathrm{mg} / \mathrm{cm}^{2}$ aluminium layer.

On the one hand, if we adopt a mean absorption coefficient, we are led to taking $\mathrm{f}_{1}=0.92$; on the other hand, the aluminium layer absorbs approximately $7 \%$ of the primary radiation; assuming that half of the fluorescence radiation is absorbed in the target itself, the contribution of this layer thus represents $\frac{0.07 \times 2 \times 3.1}{3.1}=14 \%$ of the 
emerging fluorescence radiation, and $\mathrm{f}_{1}=0.86$. It must also be pointed out that the theoretical calculation made for the stratified target would result in $f_{1}=0.90$. Figure 3 provides experimental values for $I_{(A)} / I_{(A)}$. Calculated results of the previous paragraph have been plotted on the same figure taking into account the presence of an absorbing layer of element $\mathrm{A}, 0.5 \mathrm{mg} / \mathrm{cm}^{2}$ thick, in order to render results directly comparable.

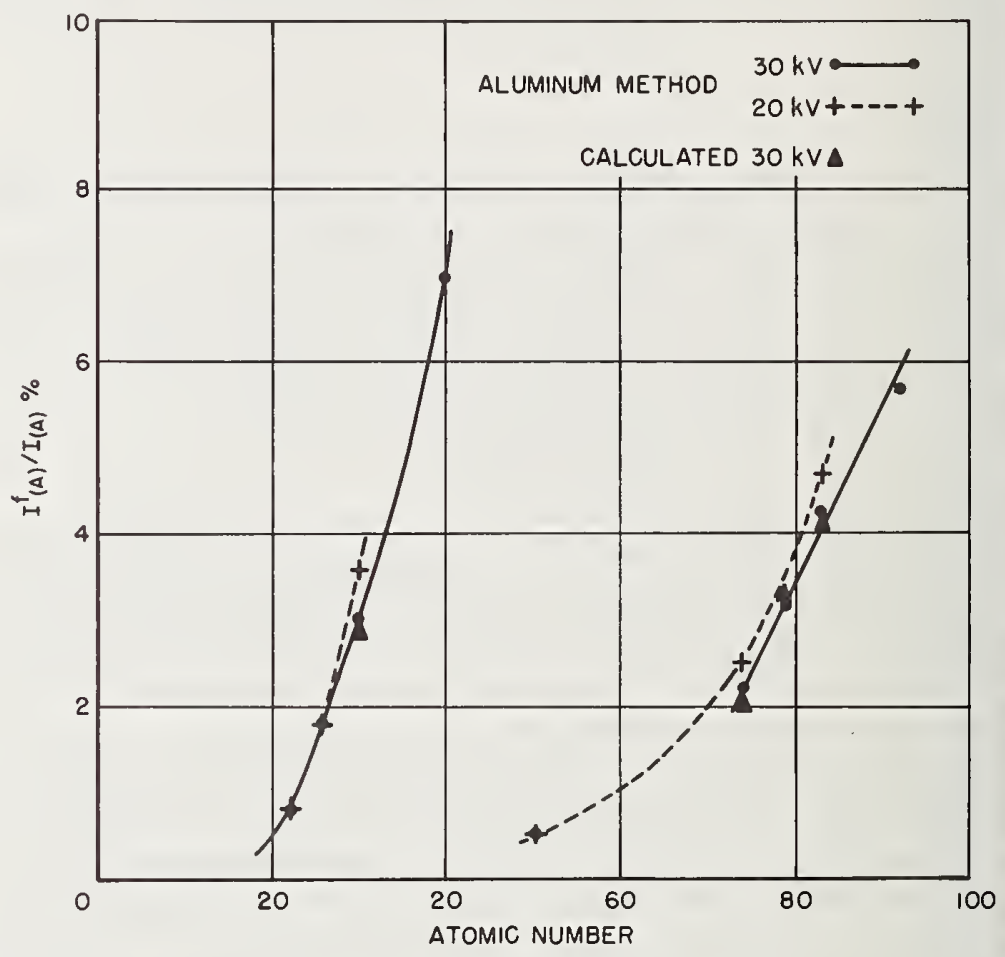

Figure 3. Ratio of indirect to direct x-ray production.

\section{DISCUSSION OF RESULTS}

From the preceding, it appears that the lack of accuracy in calculated values can be ascribed mainly to the discrepancies betweeen calculated and observed values of the characteristic primary radiation intensity. Accurate measurements of this radiation for elemental targets, though conceivable, represent a 
time-consuming, delicate, and wearisome work. Therefore, it is more advisable to have $\mathrm{I}_{(A)} / \mathrm{I}_{(A)}$ values provided by experimental methods and to calculate corrections to be made when analysing compound samples by means of tables. For the sake of exactness, imagine the case of a compound $A B$ in. which element $A$ is to be measured. For simplicity, let us assume that the other corrections are negligible. Due to the presence of the fluorescence radiation excited by the continuum, there exists between the true concentration $\mathrm{C}_{A}^{P}=\mathrm{I}_{A}^{P} / \mathrm{I}_{(A)}$ and the measured concentration $\mathrm{C}_{A}^{m}=\mathrm{I}_{A}^{m} / \mathrm{I}\left({ }_{A}^{m}\right)$, the following relation:

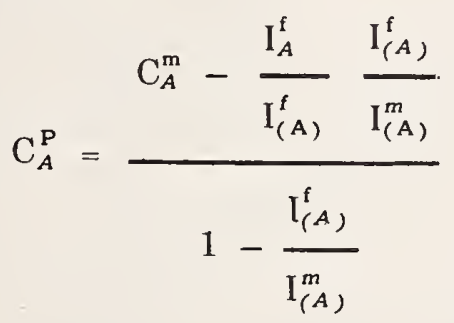

The method of calculating $C_{A}^{P}$ may be the following:

a. The terms $\mathrm{E}_{A B}$ and $\mathrm{E}_{A}$ contain only the physical constants of constituent elements and the concentration $\mathrm{C}_{A}$; their calculation offers no difficulty.

b. $\left(\phi_{m+1}-\phi_{m}\right)_{A}$ relative to the standard can be calculated by means of the table.

c. $\left(\phi_{m+1}-\phi_{m}\right)_{A B}$ relative to the specimen can be calculated by means of the table.

d. The value of the ratio $I_{(A)}: I_{(A)}$ is read from Figure 3 . Thus all quantities appearing in the right hand side of formula (12) are available. The number of experimental results readily available may seem small. Indeed they are strictly valid only for a take-off angle close to $16^{\circ}$ and for voltages of 30 and $20 \mathrm{kV}$. Attention must be drawn, however, to the fact that the take-off angle interferes only with parameter $v$ of the $\phi$ function for the fluorescence radiation. Referring to the tables we have on the one hand:

$\frac{\left.\mathrm{I}_{(A)}^{\mathrm{f}}\right] \theta_{\theta_{1}}}{\left.\mathrm{I}_{(A)}^{\mathrm{f}}\right] \theta_{\theta_{2}}}=\frac{\sum_{\mathrm{m}} \mathrm{E}_{A}^{\mathrm{m}}\left\{\Phi\left[\lambda_{0}, \lambda_{m}+1, \mathrm{v}_{A}^{\mathrm{m}}\left(\theta_{1}\right)\right]-\Phi\left[\lambda_{o}, \lambda_{m}, \mathrm{v}_{A}^{\mathrm{m}}\left(\theta_{1}\right)\right]\right\}}{\sum_{\mathrm{m}} \mathrm{E}_{A}^{\mathrm{m}}\left\{\Phi\left[\lambda_{0}, \lambda_{m}+1, \mathrm{v}_{A}^{\mathrm{m}}\left(\theta_{2}\right)\right]-\Phi\left[\lambda_{0}, \lambda_{m}, \mathrm{v}_{A}^{\mathrm{m}}\left(\theta_{2}\right)\right\}\right.}$ 
and on the other:

$$
\frac{\left[\mathrm{I}_{(A)}\right]_{\theta_{1}}}{\left[\mathrm{I}_{(A)}\right]_{\theta_{2}}}=\frac{[\mathrm{F}(X)]_{\theta_{1}}}{[\mathrm{~F}(X)]_{\theta_{2}}}
$$

is given by the absorption correction curves.

As far as the ratio $\boldsymbol{I}_{(A)} / \mathbf{I}_{(A)}$ is concerned, it remains practically constant over the range of the high voltages used.

Attention must be drawn to the fact that the formulas giving the corrections for fluorescence excited by the continuum and the characteristic radiation contain explicitly the concentration; therefore, the correction can be made only by successive approximations. As suggested by Philibert (1965) [10], a quick evaluation of the different corrections allows one to place the true concentration in a certain range; one can then establish a correspondence between true concentrations and measured concentrations by means of the correction formulas. This method can be advantageously used for fluorescence corrections by applying the formula

$$
\mathrm{C}_{A}^{\mathrm{m}}=\frac{\mathrm{C}_{A}^{\mathrm{P}}+\frac{\mathrm{I}_{A}^{\mathrm{f}}}{\mathrm{I}_{(A)}^{\mathrm{f}}} \frac{\mathrm{I}_{(A)}^{\mathrm{f}}}{\mathrm{I}_{(A)}^{\mathrm{P}}}}{1+\frac{\mathrm{I}_{(A)}^{\mathrm{f}}}{\mathrm{I}_{(A)}^{\mathrm{P}}}}
$$

\section{Conclusion}

The application of fluorescence corrections can be greatly facilitated if one has taken care to use to a maximum extent the possibilities offered by the computer; the availability of tabulated values eliminates cumbersome calculations. One may ask the question whether it is easy to forecast a priori the importance of the correction. A mere glance at formulas (12) and (13) suffices to show that the correction amount is an increasing function of the difference $\mathrm{C}_{A}^{m}-\mathrm{C}_{A}^{f}$ (or $\mathrm{C}_{A}^{P}-\mathrm{C}_{A}^{f}$ ) of the concentrations resulting from the emerging primary radiation and fluorescence radiation respectively: 
It must be emphasized that the search after a general variation law for the correction amount is rendered illusive by the fact that concentrations $\mathrm{C}_{A}^{m}$ and $\mathrm{C}_{A}^{P}$ are relating to emerging radiations. A first estimate can be quickly made if one evaluates, with the help of tables, the ratio $\mathrm{I}_{A}^{f}: \mathrm{I}_{(A)}^{f}$. A comparison between $\mathrm{C}_{A}^{f}$ and $\mathrm{C}_{A}^{m}$ (or $\mathrm{C}_{A}^{P}$ ) allows one to decide whether or not the complete calculation is necessary. In a general way, one may assume that the error in concentration introduced by the fluorescence will be less than 0.005 if $\mathrm{C}_{A}^{f}-\mathrm{C}_{A}^{P(m)}<0.05$. It must not be overlooked that the results can fluctuate by a ratio of 3:1 according to the take-off angle of the microanalyzer being used.

To conclude, we shall make reference to two instances where the ignorance of the fluorescence phenomena has led to gross errors.

When analyzing a small size precipitate situated in a matrix of a different composition, the primary radiation is that from the precipitate and the fluorescence radiation is that from the matrix. This instance was described time and again by Castaing and Descamps (1955) and by Philibert (1964). Another, practically very important instance, is that of the study of semiconductors where concentrations change quickly over a distance of only a few microns. The fluorescence radiation must then be taken into consideration in any case.

\section{References}

[1] Kramers, Phil. Mag., (1923) p. 836 jul. dec.

[2] Kulenkampff, Ann. Physik (4) 69, (1922) p. 548.

[3] Green, Thesis, University of Cambridge (England), (1962).

[4] Mott and Massey, Theory of Atomic Collisions. Clarenton Press (1949).

[5] Worthington and Tomlin, Proc. Phys. Soc. A, 69, (1956) p. 401.

[6] Whiddington, Proc. Roy. Soc. A, 86, (1912) p. 360.

[7] Listengarten, Izv. Akad. Nauk Phys. Ser. 24, (1960) p. 1041.

[8] Castaing and Descamps, J. de Phys. 16, (1955) p. 304.

[9] Kirianenko, Hénoc and Maurice, Rapport C.E.A. R.2421, (1964).

[10] Philibert, Métaux, Corrosion, Industries 40, No. $465^{\prime}$ (1964) p. 157. Métaux, Corrosion, Industries 40, No. 466 (1964) p. 216. Métaux, Corrosion, Industries 40 , No. 469 (1964) p. 325. 



\section{Symbols}

$A, B \quad$ Atomic mass of element $A, B$

$c \quad$ Constant in Thomson-Whiddington energy-loss equation

$C_{A}, C_{B} \quad$ Mass concentration of element $A, B$

$\begin{array}{ll}C_{A}^{P} & =\frac{I_{A}^{P}}{I_{(A)}^{P}} \\ C_{A}^{m} & =\frac{I_{A}^{m}}{I_{(A)}^{m}}\end{array}$

E Electron energy

$E_{i} \quad$ Exponential Integral

$E_{0} \quad$ Incident electron energy

$E_{K}, E_{L}, \ldots E_{1} \quad K, L_{1} \ldots 1$ energy level

e Electronic charge

$f_{i j} \quad$ Coster-Kronig transition coefficient

$I_{\lambda}^{c} \quad$ Continuous intensity emitted at wave-length $\lambda$

$I_{A}^{P} \quad$ Primary emerging intensity from analysed element $A$ in a compound

$I_{(A)}^{P} \quad$ Primary emerging intensity from pure $A$

$I_{A}^{f_{A}} \quad$ Fluorescence intensity emerging from analysed element $A$

$I_{(A)} \quad$ Fluorescence intensity emerging from pure $A$

$I_{A}^{m}=I_{A}^{P}+I_{A}^{f}$

$I_{(A)}^{m} \quad=I_{(A)}^{P}+I_{(A)}^{f}$

$I_{(A)}^{\prime} f$ Fluorescence intensity from element $A$ in "Aluminium Method"

$J \quad$ Mean ionization potential

$K \quad$ Constant in Kramers' formula

$\mathrm{L} \quad=$ Natural logarith $\mathrm{m}$

$n_{A} \quad$ Number of ionizations in element $A$

$N \quad$ Avogadro's number

$Q \quad$ Ionization cross-section

$r_{i} \quad$ Absorption jump ratio

$r_{k}, r_{I I I}, K, L_{1} \ldots$ absorption jump ratio

$R \quad$ Back scattering coefficient

$V_{A}^{m}=\left(\frac{\mu_{A}^{A} \operatorname{cosec} \theta}{\mu_{\lambda 0}^{\prime A}}\right)^{1 / 3} ; \quad V_{A B}^{m}=\left(\frac{\mu_{A}^{A B} \operatorname{cosec} \theta}{\mu_{\lambda 0}^{\prime A B}}\right)^{1 / 3}$

$z$

Thickness

$Z_{1}(A) \quad l$ line relative intensity

$Z_{A}, Z_{B} \quad$ Atomic number of element $A, B$ 


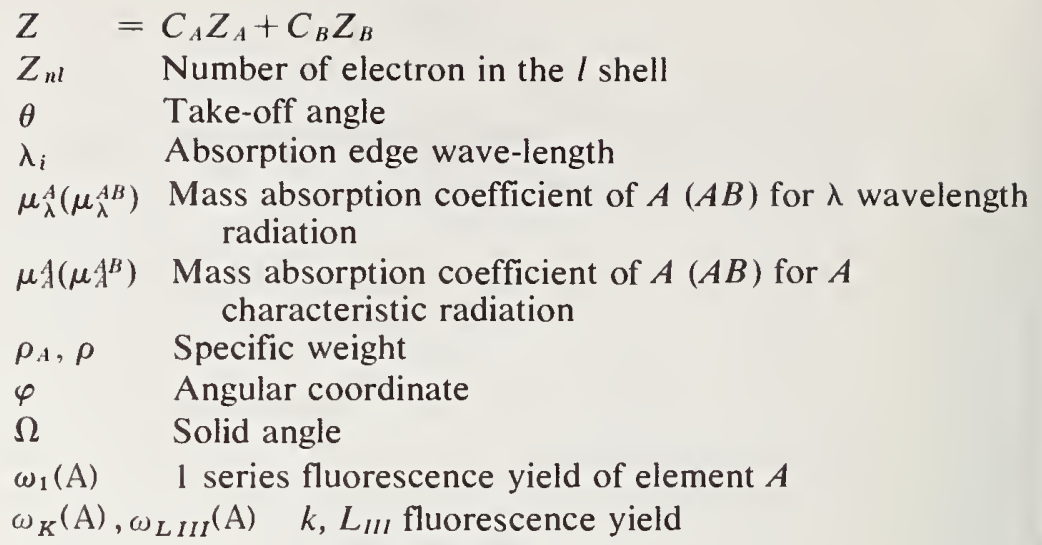




\title{
QUANTITATIVE EVALUATION METHODS FOR ALLOY MICROSTRUCTURES BY MICROPROBE ANALYSIS
}

\author{
GERHARD DÖRFLER
}

Analytisches Institut der Universitaet Wien, Austria

Abstract

The methods of stereometric analysis are of great interest in metallurgy, mineralogy, and other branches of science concerned with the properties of solid materials. Until now, practically all stereometric studies were performed by employing light microscopy.

The electron probe microanalyzer is a most valuable tool for stereometric analysis; this application has not been fully recognized as yet.

The present paper reviews the methods of stereometric analysis, and presents suggestions for further improvement of electron probe techniques specifically designed for these methods.

\section{Introduction}

During recent years, methods of stereometric analysis, called "quantitative metallography" in physical metallurgy, or "modal analysis" in mineralogy, have gained considerable interest. The general methods have been available for several decades; today, however, the far-reaching application of stereometric analysis is mainly due to the availability of automatic measuring devices.

The knowledge of structural parameters representative of the micro or macrostructure of a solid is of very great interest for solidstate research. Volume fractions, surface areas per unit volume, and grainsize distributions play an important role in the understanding of diffusion processes, reaction kinetics, homogeneity, and physical properties of solid materials. Until recently, practically all methods for measuring these quantities have employed light microscopy. A detailed discussion of all instruments presently available is given by Fischmeister (1966) [1]. In some cases, transmission electron micrographs have been used for stereometric measurements $(\mathrm{H}$. E. Exner and H. F. Fischmeister, 1966) [2]. 
Analysis by light microscopy suffers from limited spatial resolution because the reflectivity and the transmissivity of a phase is a physical property not always directly proportional to the chemical composition of this phase. A great many other factors also influence these properties strongly, e.g., orientation, surface finish in metallic systems; moreover, the etching of a sample changes considerably the correlation between chemical composition and reflectivity.

Therefore, in a great many cases, the differentiation of phases of nearly the same reflectivity is not always possible within one sample, especially when applying automatic measuring devices.

The electron microprobe has been proven to be a very valuable tool for the determination of the chemical composition of phases and it has nearly the same spatial resolution as the light microscope. This instrument, therefore, lends itself for an application in the field of stereometric analysis, especially since the scanning microprobe designed by P. Duncumb and V. E. Cosslett (1956) [3] offers the ability to scan the sample in straight lines. Thus, the most sophisticated method of stereometric analysis, linear analysis, can also be accomplished with the microprobe. It was not surprising that several attempts in this direction have been made independently by different investigators; the individual instruments will be discussed in sections III. and VI.B.2.

The present paper attempts to give a survey of the methods of stereometric analysis, and of the instruments which are presently used in combination with the microprobe, and to discuss the principles of the analytical method in detail. The usefulness of the system shall be illustrated with several recently investigated examples and by the enumeration of further possibilities of application.

In the following chapters the current state of the instrumentation will be described, with further suggestions to improve the method in the hope that this will stimulate discussion and further developments.

\section{Methods of Stereometric Analysis}

Since the first use of the light microscope for the investigation of opaque material, attempts were made to obtain not only qualitative but also quantitative knowledge of the microstructure encountered. A. Rozival (1903) [4] and E. Heyn (1903) [5] developed the first 
quantitative analytical procedures for measurements on microstructures. Subsequently, the publications of E. Scheil (1935, 1936) $[6,7]$, C. S. Smith and L. Guttman (1953) [8], S. A. Saltykov (1958) [9], J. Hilliard and J. W. Cahn (1961) [10], and others have shown that a great many parameters, representative of the threedimensional structure of opaque samples, can be obtained from measurements on randomly oriented two-dimensional planes.

Until the last decade, these methods found almost no application in the normal research laboratories. This was mainly due to the lack of automatic measuring devices as the manual measurements in stereometric analysis are extremely tedious. (Only in the last ten years have automatic measuring devices been commercially available.)

Let us now discuss the basic principles of stereometric analysis and the structural parameters which can be derived from its results. For a more thorough presentation of this subject, reference should be made to the following books: S. A. Saltykov (1958) [9], H. Elias and E. Weibel, ed. (1967) [11] and E. Underwood (1967) [12].

Two different measuring methods are known in stereometric analysis - measurements along a line, called lineal analysis - and the point-count techniques. Commmon to both methods is the fact that the results from measurements on a plane have to be related to the structure in the volume by statistical procedures. The theory of stereology ${ }^{1}$ states that either the phases or the analyzing means lines or points - have to be distributed randomly. This means that straight parallel lines or systematically arranged points can be used for the analysis only if an isotropic, statistical distribution of the phases is assumed. Otherwise the conclusions drawn from the measurements on a plane about the conditions in the total volume would be incorrect.

If the phases show an oriented distribution, a disoriented analyzing device has to be used. In lineal analysis this will be a circle since a direction change is made at each increment; in the pointcount technique, randomly distributed points are used.

In Table 1, the structural parameters and the methods by which they can be obtained are listed. According to investigations by A. Henning (1958) [13] and by J. W. Cahn (1961) [10], the pointcount technique is the most efficient method for volume-fraction analysis. However, if other parameters are needed, only lineal

${ }^{1}$ This name has been chosen by the International Society for Stereology, which deals with subjects concerning the three dimensional structure of matter. 


\section{Table 1}

$\begin{array}{lccc} & \text { Number of } \\ \text { Volume Surface/ Mean Grainsize } & \text { particles/ } \\ \text { fraction volume diameter distribution unit vol. Anisotropy Proximity }\end{array}$

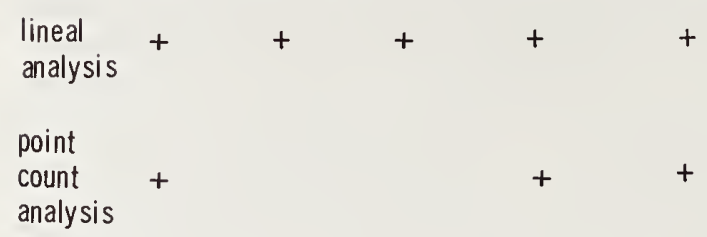

analysis can provide them. Even the simultaneous determination of all parameters during one analysis is possible by this method. The method to be chosen depends mainly on the problem to be solved.

Let us now consider the individual parameters briefly.

\section{A. VOLUME FRACTIONS}

This parameter is most commonly used in metallurgical and mineralogical investigations, because the volume fraction of a particular phase very often has a strong influence on the physical and mechanical properties of a material. The values obtained on a plane can practically always be converted to volume fractions. Furthermore, the reliability of the analysis can be given accurately as mentioned in section VIII.B. In lineal analysis, the total sum of all intercept lengths of the analyzing line falling within a particular phase is compared with the total length of the analyzing lines (Fig. 1). Applying the point-count technique, the percentage of the total points falling within the encountered phase is registered.

\section{B. SURFACE AREAS PER UNIT VOLUME}

The knowledge of the surface areas of individual phases is naturally very important to the understanding of diffusion processes, kinetic processes, grain growth, etc. Furthermore, it has been shown by several authors (Saltykov, 1958) [9] that the amount of surface area present in a solid very strongly influences several physical properties such as electric conductivity, hardness, and tensile strength.

For the calculation of the surface area per unit volume, it is necessary to register not only the sum of all intercept lengths but 


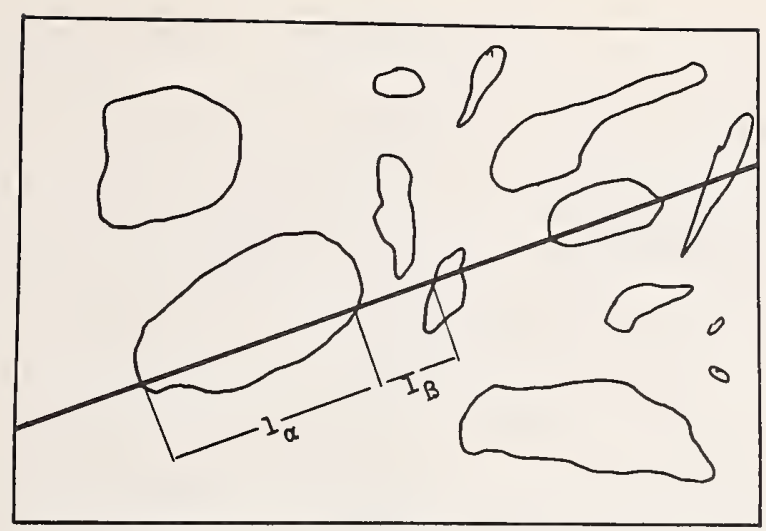

Figure 1. Principle of lineal analysis: $1_{\alpha}=$ intercept length of phase $\alpha ; 1_{\beta}=$ intercept length of phase $\beta$.

also the number of intersections with that particular phase; i.e.,
$\mathrm{S}_{V}=\frac{2 \mathrm{n}_{L}}{\mathrm{~L}}=\frac{2}{\mathrm{~L}}$
$\mathrm{S}_{V}=$ surface area $\left(\mathrm{mm}^{2} / \mathrm{mm}^{3}\right)$
$\mathrm{n}_{L}=$ number of intercepts
$\underline{L}=$ length of test line within the phase
$\overline{\mathrm{L}}=$ mean grain diameter

\section{MEAN DIAMETER OF A PHASE}

This value can be calculated from the same data used for the surface areas:

$$
\overline{\mathrm{L}}=\frac{\mathrm{L}}{\mathrm{n}_{\mathrm{L}}}
$$

This provides a very convenient means for the rapid and simple characterization of grainsize.

\section{PROXIMITY}

This parameter has been introduced very recently to stereology by the author (Dörfler, 1966) [14]. It makes an attempt to quantitatively characterize the arrangement of phases, which was not possible up to now. Figure 2 shows the basic principle for a three-phase sample. From the number of sequences and the intercept lengths of the individual phases, the proximity of one phase respective to all others can be calculated. In the example schematically shown in Figure 2, it can be seen that in case a) all the 

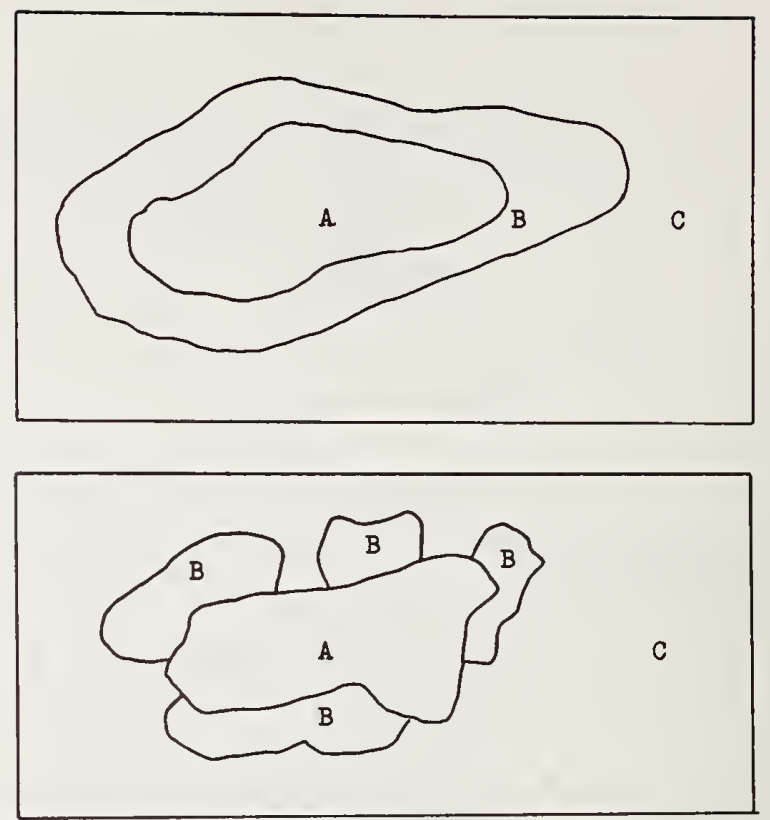

Figure 2. Principle of sequence analysis: 2a: Phase A totally surrounded by phase $\mathrm{B}$, Proximity $\mathrm{P}_{A / B}=100 \%, \mathrm{P}_{A / C}=0 \% ; 2 \mathrm{~b}$ : Contacts between phases $\mathrm{A}, \mathrm{B}$, and $\mathrm{C}$, $\mathrm{P}_{A / B}=80 \%, \mathrm{P}_{A / C}=20 \%$.

surface area of phase $\mathrm{A}$ is in direct contact with phase $\mathrm{B}\left(\mathrm{P}_{A / B}=\right.$ $100 \%)$ while no contact area exists between $\mathrm{A}$ and $\mathrm{C}\left(\mathrm{P}_{A / C}=\mathrm{O}\right)$. In case b) $80 \%$ of the total surface area of $\mathrm{A}$ has contact with $\mathrm{B}\left(\mathrm{P}_{A / B}=\right.$ $80 \%)$, the remaining $20 \%$ being surrounded by the phase $\mathrm{C}\left(\mathrm{P}_{A / C}=\right.$ $20 \%$ ). It is easily recognizable that the knowledge of this parameter is very important for quantitative calculations in reaction kinetics and diffusion processes. The sequences can be registered automatically by means of the sequence analyzer (Dörfler, 1966) [15], which will be described in section VI.C.3.

$$
\mathrm{P}_{A / B}=\frac{\mathrm{S}_{A / B}}{\mathrm{~S}_{A}} \cdot 100(\%)
$$

$\mathrm{P}_{A / B}=$ proximity of phase $\mathrm{A}$ to $\mathrm{B}$

$\mathrm{S}_{A / B}=$ surface area of phase $A$ in contact with phase $B$

$\mathrm{S}_{A}=$ total surface area per unit volume of phase $A$ 
Both parameters are very important for detailed investigations in the field of nucleation and grain growth, as well as for the accurate description of microstructures. However, the methods for the analysis and for the calculation are rather complicated and cannot be discussed in detail in this paper. (Refer to section VI.C.4.)

Very detailed discussions of the problem can be found in the papers of E. Scheil $(1935,1936)$ [6,7], S. A. Saltykov (1958) [9], R. T. DeHoff $(1962,1965)[16,17]$, H. E. Exner $(1966)[18,19]$ and G. Bockstiegel (1966) [20]. ${ }^{2}$

The determination of the number of particles per unit volume is even more complicated and depends on a knowledge of the shape of the encountered particles. Discussions and references can be found in the above mentioned papers.

Other parameters, such as anisotropy, topological parameters (continuity, connectivity) etc., can be determined by these methods; and it is felt that additional parameters would be necessary for a representative description of a microstructure.

\section{Automatic and Semiautomatic Devices, Using the Electron Probe}

The advantages of the use of the electron microprobe for stereometric analysis has been pointed out in section I.

In 1964, two similar arrangements were proposed: R. Theisen [21] modified a line-recorder to operate as a switch, which allowed the counting of the number of interested inclusions, provided that the concentration of a certain element exceeded a preset level. In combination with a special $\mathrm{x}$-ray pulse-count technique, Theisen obtained the mean particle size and anisotropy value for $\mathrm{FeAl}_{3}$ inclusions in SAP (sintered aluminium powder).

D. A. Melford and K. R. Whittington (1964) [22] published at the same time the development of an "inclusion counter" which was mainly based on a computer technique, which analyzed the x-ray and backscatter output of the microprobe. The phases or inclusions are distinguished by the presence or absence of three elements ( $\mathrm{x}$ ray analysis) and by the value of the mean atomic number as given by the intensity of the backscattered electrons. If a phase is identified, sizing circuits are activated, which record the number of

${ }^{2}$ For a conversion into a size distribution in space, a convex shape of the particles has to be assumed. Some older theories deal only with the distribution of ideal spheres. 
intercepts falling into a special size class. A special provision is made to avoid the counting of an inclusion twice while analyzing on subsequent lines. This method provides an extraordinarily high speed, the analysis on a field of $500 \times 500 \mu^{2}$ being performed in 30-60 seconds (Figure 3).

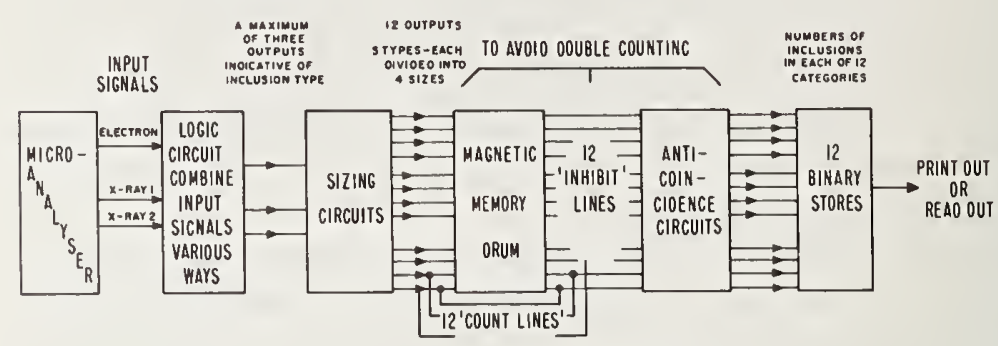

Figure 3. Schematic diagram of the "inclusion counter" (Melford and Whittington, 1966).

However, the method of Theisen as well as the "inclusion counter" are restricted to the analysis of phases, which differ qualitatively (by the presence or absence of at least one element) from the matrix. If the same elements are present in two phases, but only in differing concentrations (e.g., ferrite/austenite), these instruments cannot distinguish between them.

In 1965 two further instruments were introduced by P. Weinstein [23] and by G. Dörfler [24]. Though they use very similar principles, they have been developed independently. Their arrangements have been strongly influenced by the development of discrimination circuits for the improvement of x-ray scanning, published by K. F. J. Heinrich (1962) [25], D. A. Melford (1962) [26] and M. Rouberol et al. (1962) [27]. Since the two instruments are so similar, they can be discussed as one system.

The basic principle of the apparatus, called "phase integrator", is shown in Figure 4. A signal of the electron microprobe (x-ray, target current, etc., ref. to section V.) is fed into a discriminator circuit, where the identification of the selected phase is accomplished. If the signal voltage remains within the lower and the upper threshold limits, a constant voltage is supplied which opens a gate. Through the open gate, pulses of constant frequency, which are produced by an external oscillator, can pass to the analyzing circuits. The pulse groups obtained by this way represent the intercept length of the 


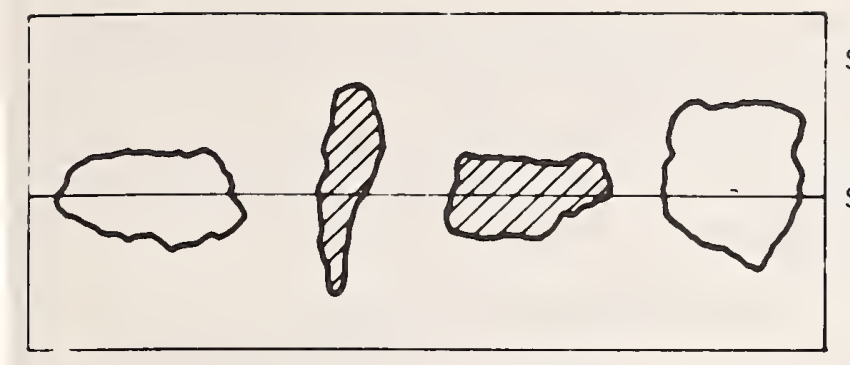

scanning area

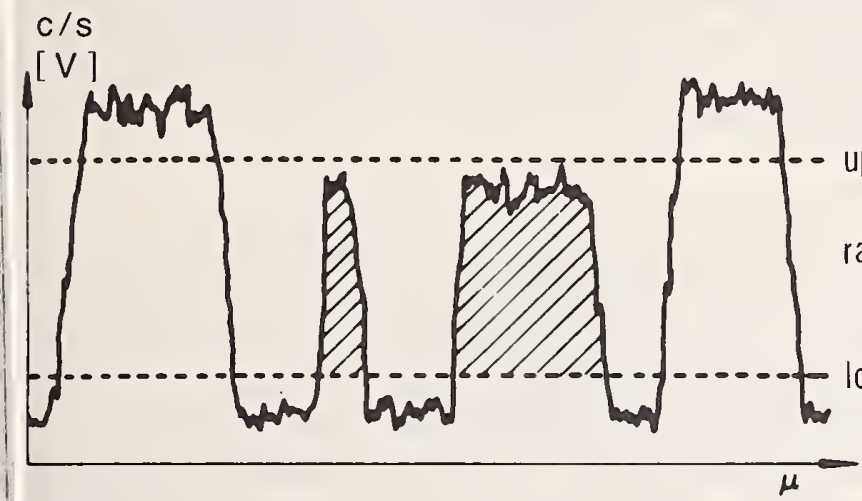

A

$\left\{\begin{array}{l}c / s \\ {[V]}\end{array}\right.$

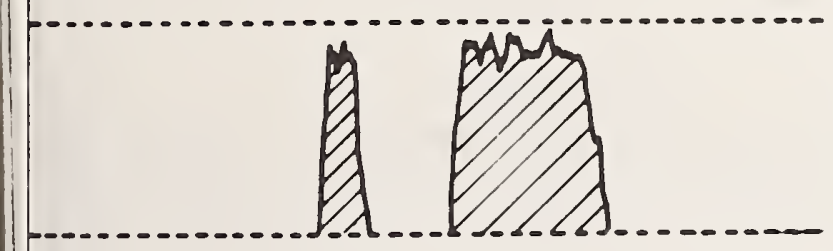

scanning line

upper threshold

ratemeter voltage

lower threshold

B

discriminated ratemeter voltage

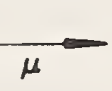

C

[V]

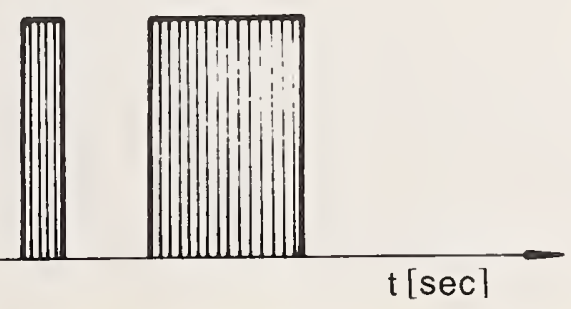

D

Figure 4. Principle of the "Phase Integrator" (Dörfler and Plöckinger, 1966). 
linear analysis and can, therefore, be analyzed to give all structural parameters desired. A detailed discussion of the discrimination circuits is given in section VI.B.

The same possibilities for obtaining stereometric data have been pointed out recently by K. F. J. Heinrich (1966) [28], in an expansion of the concentration mapping device.

\section{Basic Techniques of Analysis}

Since most operations are common to all techniques, the basic procedures shall be discussed in this section. Not only the present state of knowledge, but also some suggestions for further improvements will be discussed.

\section{A. THE SCANNING PROCEDURE}

The basic technique of scanning a focused electron beam on a sample was originally developed by M. von Ardenne (1938) [29] for his scanning electron microscope. However, this technique found no far-reaching application until P. Duncumb and V. E. Cosslett (1956) [3] introduced it into electron-microprobe analysis. The great advantages and the limitations of this technique have been thoroughly discussed several times, recently in great detail and with special reference to the quantitative interpretation of data by K. F. J. Heinrich (1967) [30].

\section{Lineal Scanning}

Practically all scanning devices included in commercially available electron probes shift either the electron beam or the specimen in two orthogonal lineal movements. The displacement in the $\mathrm{x}$-axis is normally by a factor of 10 or 100 faster than the shift in the $y$-axis.

Let us now consider the features which should be included in a universal scanning device in order to fulfill all requirements of stereometric analysis; these are strongly influenced by the analytical problem to be solved. Figure 5 gives a general impression of the relationships. Depending on the grainsize and the distribution of the phases, a proper line length has to be chosen. The distance between two subsequent lines is also affected by the distribution of the phases, in order to give a representative number of particles. The number of lines has to be selected to give the accuracy required for the analysis (ref. section VIII.B.). 


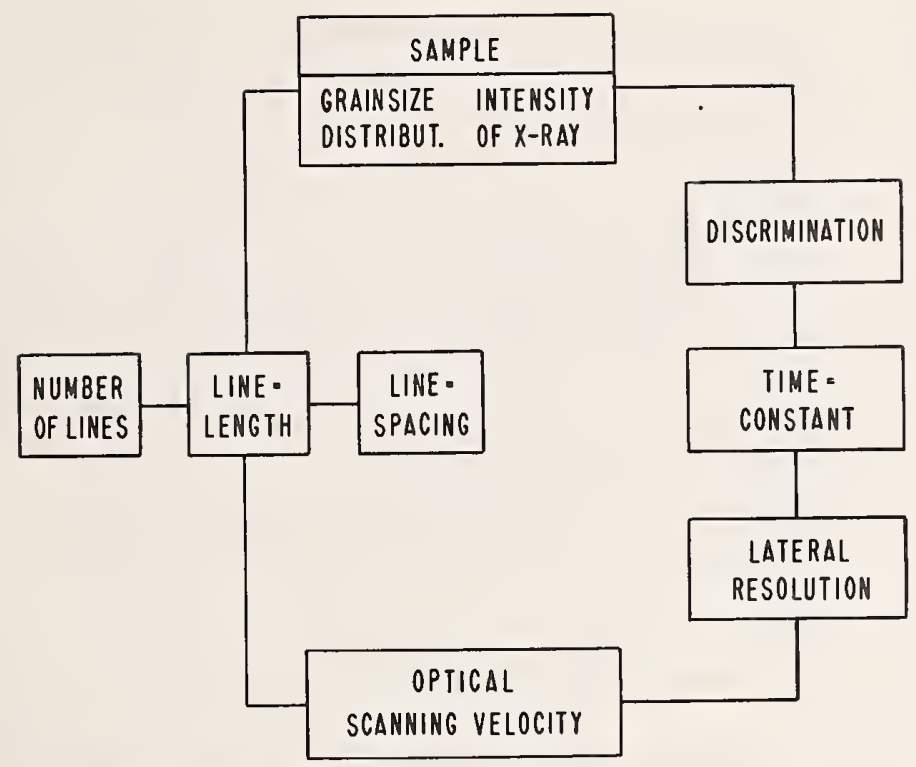

Figure 5. Best way of selecting the scanning parameters.

Thus it should be possible to select line length, spacing, and the number of lines independently of each other. This is, however, nearly impossible in commercially available scanners. In these, the scanning field is normally a square, the side length of which is set by the magnification control. Furthermore, both motions, for the lineand for the frame-formation, are continuous. These facts mean that the above mentioned parameters can not be selected independently. This can be demonstrated by the assumption that the motion vertical to the line scan is in the same order of magnitude as the horizontal. Figure 6 shows an extreme case. It is apparent that the square is distorted, which may influence strongly the analysis, if the anisotropy or orientation of a phase is determined.

In Figure 7, an arrangement is proposed (G. Dörfler, 1966) [15], which includes all features demanded above. The $\mathrm{x}$-deflection of the electron beam is directed by a sawtooth generator (1), the peak-topeak voltage of which can be varied within a wide limit by means of a precision potentiometer. This voltage is supplied to the scanning plates of the microprobe and the CRT. To obtain a stepwise deflection in the $y$-direction, the sawtooth is differentiated in (2). Each end of a scanning line is therefore marked by a pulse. In (3) 


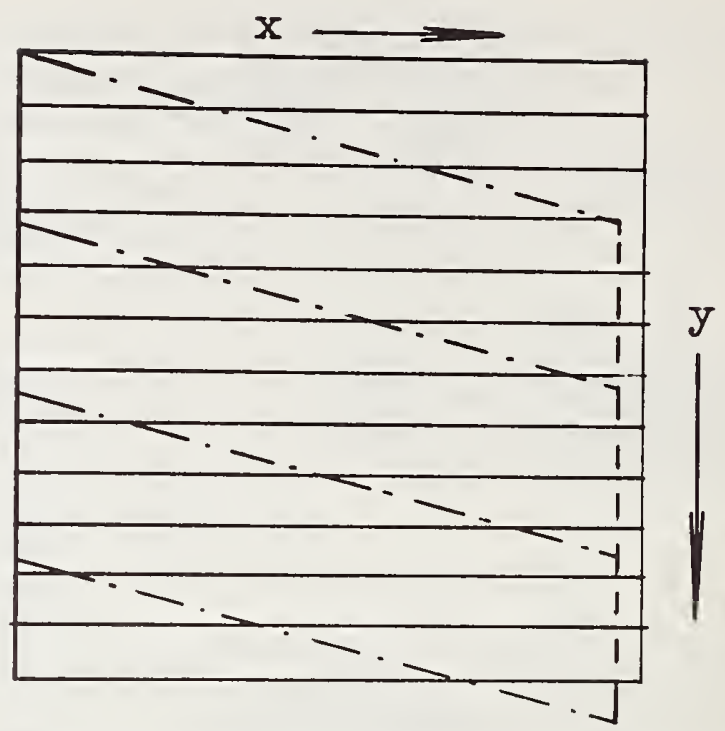

Figure 6. Influence of a continuous movement in the y-direction of the scan on the shape of the scanned area.

this pulse is formed into a square wave which has a well-defined charge. This pulse charges a loadsensitive amplifier which consists of a potentiometer (4), a differential amplifier (5) and a capacitor (6). At the potentiometer (4), the load supplied to the amplifier is adjusted; this regulates the voltage obtained at the ends of the capacitor. Since this voltage is supplied to the deflection plates of the microprobe and the CRT, the resistor (4) determines the distance between one line and the next. Each step in the y-direction is registered by the preset counter ( $\mathrm{ZW}$ ). If the preset number of lines is reached, the counter generates a pulse which actuates the AND-gate (7) and thereby shortcircuits the capacitor (6). This results in the resetting of the $y$-voltage to the initial value at the first line. Thus, by this device, the line length can be selected at the sawtooth generator (1), while the linespacing is determined by the position of the potentiometer (4). The $y$-motion is triggered by the steep slope of the sawtooth, i.e., the line end. Therefore the line length, the scanning speed and the line spacing can be selected independently.

The y-motion can consist either of a deflection of the electron beam or of a displacement of the sample. Instead of the above 


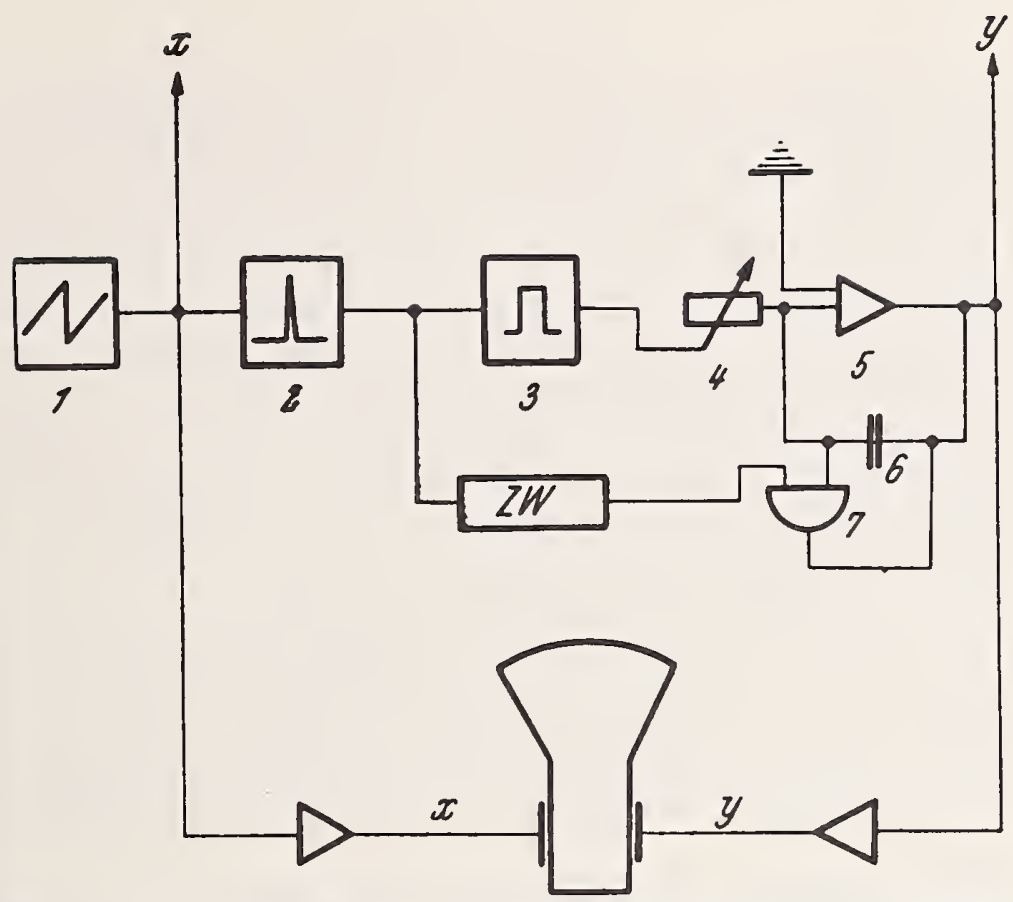

Figure 7. Proposed arrangement for step-scanning in the y-direction: $1=$ Sawtooth generator; $2=$ differentiator; $3=$ pulse shaper; $4=$ potentiometer for step-height selection; 5=operational amplifier; 6=capacitor; 7=AND-Gate; $\mathrm{ZW}=$ preset counter (selection of the number of steps).

mentioned charge-sensitive amplifier, a digital-to-analog converter could be used to produce the stepwise y-motion. The two potentiometers at (1) and (4) can be calibrated (ten-turn potentiometers) to enable the selection of a certain line length or spacing at a given accelerating potential. It is very important in stereometric analysis to work with exactly defined line lengths because several parameters (grainsize, grainsize distribution, etc.) depend in their precision on the accuracy of the line length measurement.

In addition to the necessity of an extremely flexible scanning device, provision should be made to scan several areas on the same sample automatically and sequentially. By means of an electromechanical step-scanning device, one field can be linked up with the next, enabling the accurate exploration of large areas and the programmed search for unknown or scarce phases. 


\section{Point-Scanning}

This technique has been recently introduced into microprobe research by L. S. Birks (1963) [31], who used a multichannel analyzer for the convenient storage of quantitative point counts distributed on a grid, scanned by the electron beam on the sample surface. K. F. J. Heinrich (1966) [28] also described this possibility, using a 1600 channel analyzer and showing not only the digital printout, but also the visual display on the CRT, where the "threedimensional" presentation is very instructive. The paper-punch and magnetic-tape readout facilities of these systems allow a subsequent analysis of the data in a computer. (K. F. J. Heinrich, private communication.) Some possibilities for the application to stereometric analysis are discussed in section VI.D.

The first system directly devoted to stereometric analysis was proposed by K. Keil (1965) [32]. He used an electro-mechanical step scanner to obtain a systematic point scan. At each point, the signals for three $\mathrm{x}$-ray spectrometers, the target current, and the cathodoluminescence were used to decide which phase is present. The decision can be made either by the operator or by an automatic device.

Generally, the point-count method has the disadvantage that not very many parameters can be determined by this technique (ref. section II.). However, this method seems to be extremely valuable for the discrimination of phases with very small concentration differences because a preselected statistical reliability can be obtained by using the fixed-count method. A further application of the point-count method is the determination of the homogeneity of single-phase or multiphase samples (K. F. J. Heinrich, private communication).

\section{Signals of the Microprobe and Their Use for Stereometric Analysis}

To obtain a stereometric analysis by means of the microprobe, all signals obtained while scanning over the sample can generally be used. It is very important to point out that for this type of analysis no quantitative determinations are needed. It is only necessary that the signal differences between the phases present be great enough to make an accurate discrimination possible. This causes some points of view which are partially different from the normal discussion of the signal sources of the electron microprobe. 


\section{A. X-RAY SIGNALS}

Originally, the microprobe was designed for the determination of the chemical composition of microvolumes by means of the characteristic $\mathrm{x}$-ray emission. Since the chemical composition is in nearly all cases the most selective means for a discrimination, the detection of the characteristic x-radiation should be discussed in detail. It must be mentioned, however, that not only the characteristic radiation, but also the intensity of the white radiation can be used to distinguish between different phases (F. R. Park et al. 1966) [33]. These authors obtained a resolution of 2.5 atomic numbers at a wave length of $0.60 \AA$ and $30 \mathrm{kV}$ acceleration potential. When using characteristic $\mathrm{x}$-rays in scanning-microprobe analysis, and especially in stereometric analysis, some characteristics caused by the quantum nature of the $\mathrm{x}$-rays have to be considered. This may be illustrated by an example. If two phases are present in a sample, one of which contains $20 \%$ and the second $25 \%$ of a certain element, the coefficient of variation has to be at least $0.1(=10 \%)$ to make a discrimination possible. Applying pulse-counting statistics, the coefficient of variation is given by the formula

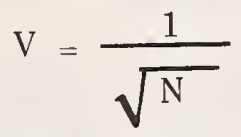

From this it can be calculated that at least 100 counts have to be accumulated on the phase containing $20 \%$ (100 \pm 10 counts). Therefore $120 \pm 11$ pulses will be obtained on the $25 \%$ phase.

For an accurate discrimination, a confidence limit of $68.3(1 \sigma)$ will not be sufficient. Therefore, very high counting rates should be used for the purposes of stereometric analysis whenever possible.

In the case of a point-count analysis, the coefficient of variation to be reached can be obtained by the proper selection of the number of counts accumulated (fixed-count method). If, however, lineal analysis has to be applied, the integration time is given by the time constant of the ratemeter $(\tau)$ which acts as a digital-to-analog converter. With the time constants normally used for lineal analysis (10 msec $-1 \mathrm{sec}$ ), the actual counting rate is considerably reduced, e.g., for $=0.1 \mathrm{sec}$ to $10 \%$ of the count rate.

If the periodic pulse integrator proposed by K. F. J. Heinrich (1964) [34] is used, the integration time is much better defined than that of a ratemeter. The ratemeter and the pulse integrator will be discussed in more detail in section VI.A. 
From these considerations it can be concluded that, depending on the discrimination problem, in most cases a high counting rate, and therefore better statistics, is more favorable than high peak-tobackground values. Therefore, if applicable, nondispersive counting systems will give much better results in stereometric analysis than dispersive systems. This is especially true if the fulfillment of the Rowland circle condition is considered. When scanning the electron beam on the sample, the focusing conditions of fully-focusing spectrometers are fulfilled only along a line, depending on the characteristics of the spectrometer. If the beam is deflected in a direction normal to that line, a considerable loss of counts can be observed (H. Malissa, 1966) [35]. As K. F. J. Heinrich (1967) [30] has pointed out, this can be avoided in the following ways:

1. To restrict the width of scanning (smaller than $50 \mu \mathrm{m}$ ). This method is not applicable for stereometric analysis.

2. To use scanning by mechanical displacement. This method is especially suitable for stereometric analysis, because the pulse rates obtainable with dispersive methods permit in most cases only slow scanning velocities.

3. To employ signals other than $\mathrm{x}$-rays. This possibility will be discussed in the following section.

4. To use semifocusing or nondispersive $\mathrm{x}$-ray spectrometers. Since nondispersive methods give higher counting rates up to a factor of 1000 (P. Duncumb, 1964) [36], they are especially suitable for our demands. A further improvement could be obtained by applying the recently developed solid-state $\mathrm{x}$-ray detectors, which show an exceptionally good energy resolution and are capable of analyzing radiations down to $\mathrm{TiK} \alpha$. Since these detectors are very small, they can be placed near the sample at a high take-off angle.

\section{B. TARGET CURRENT}

The fraction of the beam current absorbed by the specimen is a function of the mean atomic number of the encountered area and of the surface topography. Analyzing a well-polished sample, the target-current variations can be attributed to the atomic-number variations. If the differences in atomic number of the phases present are great enough, the selection of these phases can be very easily made by the target current. This signal has the very great advantage that it has a very small time constant and that the spatial resolution 
is better than that of the x-ray signals. Therefore, a much higher scanning speed can be obtained. Very detailed accounts on the theory and the use of the target current have been given by $\mathrm{E}$. Weinryb (1964) [37] and by K. F. J. Heinrich (1964) [38].

\section{BACKSCATTERED ELECTRONS}

Backscattered electrons consist of two main classes, the elastically scattered electrons and the secondary electrons. The elastically scattered electrons show the same dependence on the atomic number of the target as the target current. However, the development of a "stereo monitor" (S. Kimoto and H. Hashimoto, 1966) [39] permits the separation of atomic number and topography effects. The principle is demonstrated by two illustrations from the above mentioned paper (Figures 8 and 9). Two solid-state electron detectors are used. If the sum of both signals is used, all topographic effects are eliminated and only the atomic-number signal is obtained. In the subtraction mode, the topography signal can be observed.

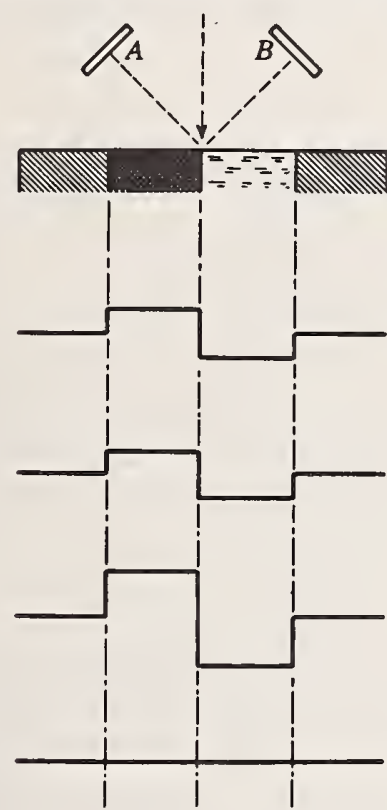

(a)

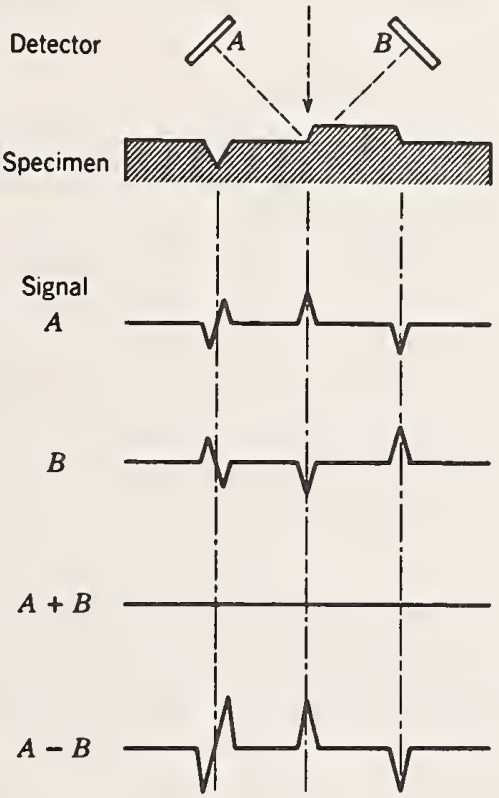

(b)

Figure 8. Signals from multiple detectors and their combination for simple specimen (Kimoto and Hashimoto, 1966). 


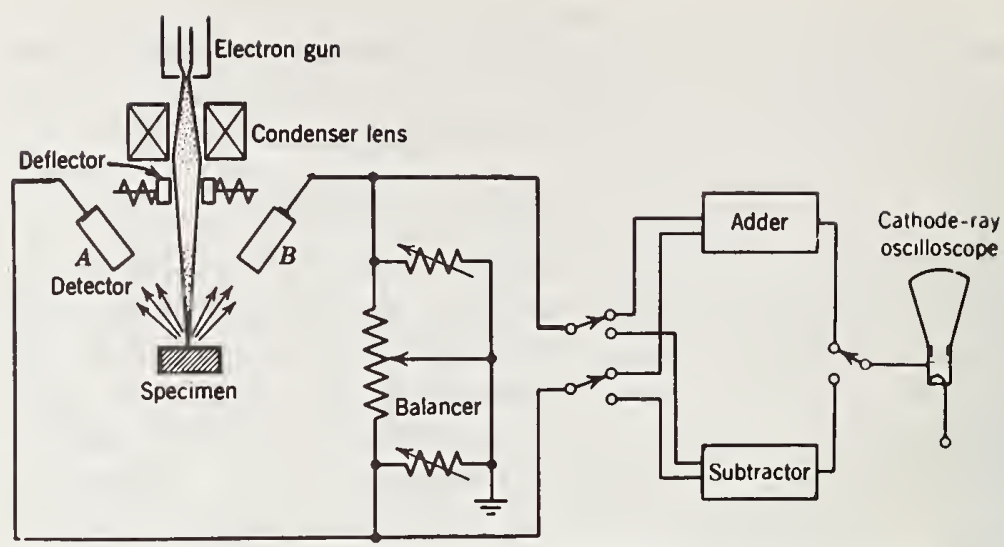

Figure 9. Arrangement of the "stereo detector" (Kimoto and Hashimoto, 1966).

For stereometric work, both signals can be used. In the "composition mode" it is possible to get rid of all topography effects, in the "topography mode" the structural parameters of pores, cracks and grain boundaries (if etched) can be evaluated.

Not very much is known about the applicability of secondary electrons to the characterization of phases. It may be that there are some very useful applications for phase discrimination.

\section{CATHODOLUMINESCENCE}

Light emission under the irradiation of the electron beam has been shown to be a very valuable tool for the rapid identification of mineral phases. Table 2 gives some observations compiled from the literature (K. Keil, 1966) [40]. The use of monochromators and a photomultiplier should enable a very rapid stereometric analysis of several mineral phases. The spatial resolution of the method is excellent (K. F. J. Heinrich, 1967) [30].

An extremely interesting development has been published by $\mathrm{D}$. Kyser and D. Wittry $(1964 ; 1966)[41,42]$. These authors recorded the infrared luminescence caused by the beam-irradiation on semiconductor specimens. Since intensity variations can be attributed to variations in the concentration of impurities or crystal imperfections, this seems to be a way to obtain stereological parameters where no other method is applicable. 
Table 2

\begin{tabular}{|c|c|c|c|c|c|}
\hline \multicolumn{6}{|c|}{ Color } \\
\hline Mineral & Blue & Red & Orange & Yellow & Green \\
\hline enstatite $^{a}$ & + & + & & & \\
\hline plagioclase & + & & & & \\
\hline oldhamite & & & & + & \\
\hline quartz & & & + & & \\
\hline sinoite & & & & & + \\
\hline $\begin{array}{l}\text { calcite } \\
(0.13 \mathrm{Mn} / 0.2 \mathrm{Fe})\end{array}$ & & & + & & \\
\hline $\begin{array}{l}\text { dolomite } \\
(0.07 \mathrm{Mn} / 0.8 \mathrm{Fe})\end{array}$ & + & & & & \\
\hline
\end{tabular}

\section{Analyzing Circuits}

The characteristic signals mentioned in the preceding section have to be analyzed in order to permit an exact phase identification and to obtain appropriate readings which can be converted to stereometric parameters.

Besides the ratemeter and the pulse integrator, proposed by K. F. J. Heinrich (1964) [34], no other instruments, incorporated in the microprobe to detect and amplify signals, shall be discussed here.

\section{A. RATEMETER AND PULSE INTEGRATOR}

If lineal scanning methods are employed, a digital-to-analog converter has to be used to produce a continuous signal while registering $\mathrm{x}$-ray pulses. These integrating devices have certain characteristics which largely influence the accuracy of the phase discrimination.

In the conventional ratemeter, a combination of a resistor and a capacitor is used for the integration (Figure 10a left). With this arrangement, a sudden change in the counting rate does not result 
in a sudden change of the ratemeter voltage but is smoothened by the time constant $(\sigma=\mathrm{R} \cdot \mathrm{C})$. Figure 11a (right) shows the effect of the time constant on the output voltage after a sudden rise in the pulse rate. It can be seen that after $4 \sigma$ the maximum value is nearly reached. The attainment of the maximum value however is absolutely necessary to obtain quantitative results (ref. section VII.). Therefore, the time constant of the ratemeter is a serious limiting factor for the scanning speed and the spatial resolution.

The periodic pulse integrator (K. F. J. Heinrich, 1964) [34] seems to be a great improvement in this respect. The principle is given in Figure $10 \mathrm{~b}$. At the output, a "block diagram" is obtained, the width of each block depending on the time of the storage cycle (t), and the height being proportional to the number of counts accumulated during the cycle. The great advantage of this system is the fact that the integrator disregards all information stored before the integration cycle. By this way, the "tailing effect" of a conventional ratemeter is avoided. Furthermore, the integration time, which determines the statistical accuracy of the pulse-rate determination, is much better defined than by the time constant of a ratemeter.

\section{B. DISCRIMINATOR CIRCUITS}

Discrimination units have been well known for a long time and have been applied to energy analysis as well as to threshold switches. As was mentioned earlier, these circuits have been introduced to electron microprobe work by K. F. J. Heinrich (1962) [25], D. A. Melford (1962) [26], and M. Rouberol (1962) [27].

As can be seen from Figure 4, the signal voltage is fed into a discrimination circuit, where a lower and an upper threshold limit can be selected. If the signal voltage obtains values between the two selected threshold settings, a constant voltage is available at the output of the discriminator. If, however, the signal level is below the lower or above the upper threshold limit, no voltage is obtained at the output of the discriminator. This means that the analog signal is converted into a digital "yes or no" decision, determining whether the phase is present or not.

This method has been developed to a very sophisticated degree by K. F. J. Heinrich $(1962,1964,1966,1967)[25,28,30,34]$ and can analyze all signals mentioned in section V. 

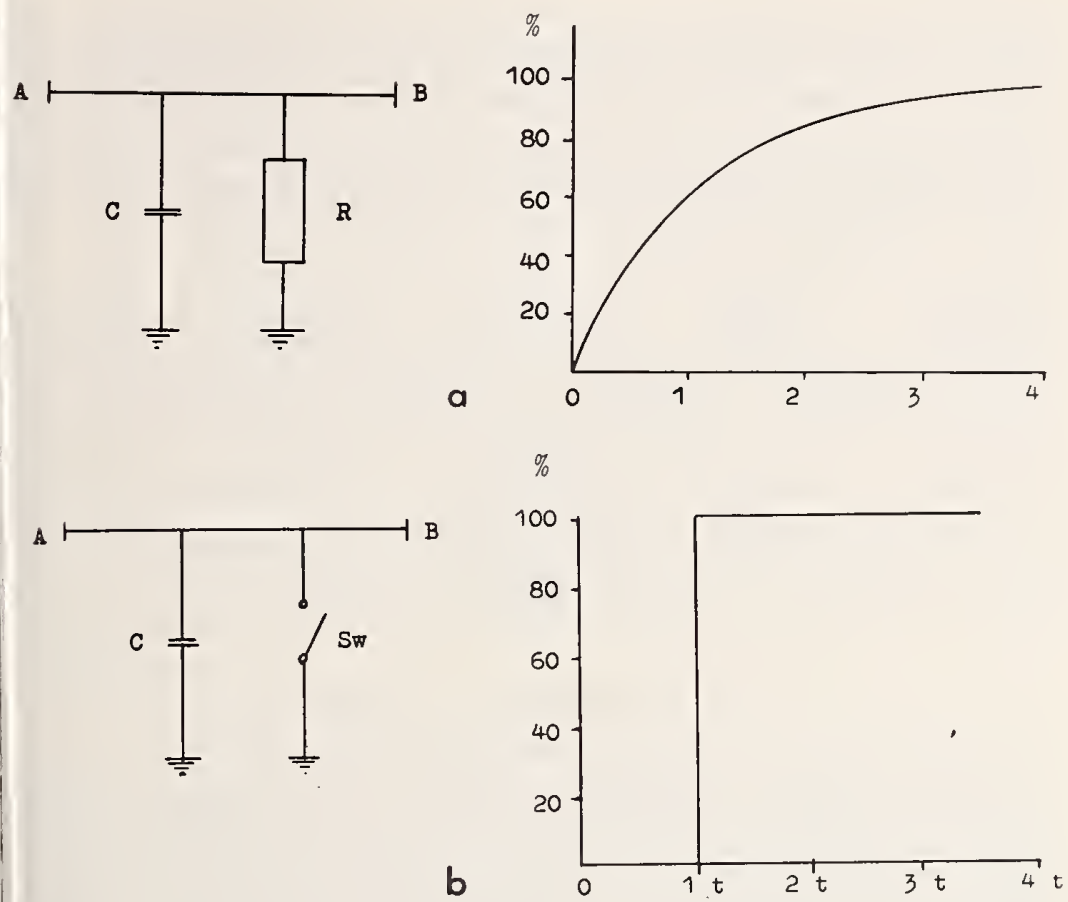

Figure 10. Basic differences between the ratemeter and the periodic pulse integrator; $10 \mathrm{a}$ : Diagram and time-response to a sudden rising voltage of a conventional ratemeter, $10 \mathrm{~b}$ : Diagram and time-response of a periodic integrator.

The output voltage can be used to open a gate, which allows the passage of external pulses. In the systems of M. Rouberol (1962) [27] and D. A. Melford (1962) [26], x-ray pulses passed through the gate to the scanning oscilloscope, thus giving an expanded contrast $\mathrm{x}$-ray scanning.

\section{Discriminators in Lineal Analysis}

In stereometric analysis an external pulse generator produces pulses of constant frequency which pass through the gate to the analyzing circuits. The measurement of the areal fraction of a phase is a time measurement determining how much of the total analyzing time the electron beam spent within the phase encountered. If, for 
example, an oscillator frequency of $100 \mathrm{c} / \mathrm{s}$ is chosen, 60,000 pulses will be produced during 10 minutes of analysis. If, on the other hand, a counter connected with the gate registers only 30,000 pulses during the same analysis, it can be concluded that the electron beam was within this particular phase $50 \%$ of the time. Therefore, $50 \%$ of the scanned area consists of this phase. (Figure 11)

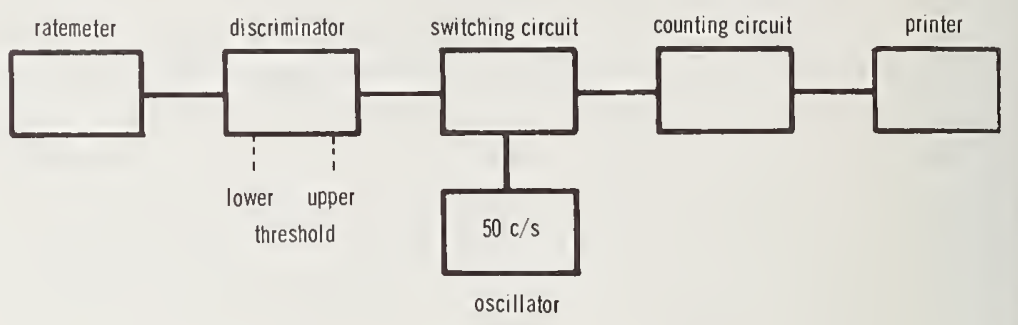

Figure 11. Arrangement of the "Phase Integrator".

Several parallel discriminator units can be used for the simultaneous determination of all phases present in the alloy. In this case it is also possible to connect several discriminators with one signal source (e.g., ratemeter) to select several concentration ranges of one element (G. Dörfler, 1966) [14].

A further possibility is the introduction of coincidence circuits ( $G$. Dörfler, 1965) [24]; (K. F. J. Heinrich, 1967) [30]. In the case of very similar phase compositions, the restriction that the conditions for two different signal discriminators have to be fulfilled yields a great improvement.

\section{The "Inclusion Counter"}

Despite the advantages of lineal analysis, the "inclusion counter" of D. A. Melford and K. R. Whittington $(1964 ; 1965)[22,43]$ shall be discussed in this section separately because of its very sophisticated design.

This instrument uses simple discriminators and coincidence circuits for a very rapid determination of the grainsize distribution of inclusions in steel (Figure 3). Since the instrument is mainly devoted to a rapid determination of these parameters, considerable reductions in the selectivity of phases have to be admitted. Using $\mathrm{x}$ - 
ray signals, the statistical limitations mentioned in section $V$.A. have to be taken into consideration. Applying scanning speeds of 500$1000 \mu \mathrm{m} / \mathrm{sec}$ (e.g., $500 \mu \mathrm{m}$ line, 100 lines in $60 \mathrm{sec}$ ) and normal pulse rates, only the presence or absence of elements is clearly detectable. Therefore, Melford and Whittington used a further signal - the number of the backscattered electrons - to get a good selection of inclusions with low atomic number. The four signals (three $\mathrm{X}$-ray and one atomic-number signal) are fed into discriminators and subsequently into coincidence circuits. A particular phase is defined by the presence or absence of several selected elements and by a certain atomic number. The signals, passing the coincidence circuits and representing the intercept length, are analyzed in a sizing circuit, the construction of which was not described in the papers mentioned above. The signals obtained from the sizing unit represent four grainsize groups of three types of inclusions.

Provision is made that each inclusion is counted one time only to obtain the exact number of particles in the scanned area. However, this does not seem to be necessary if the number of particles per unit volume is desired because this parameter is calculated from the distribution of the intercept length only (H. E. Exner, 1966) [18,19], (G. Bockstiegel, 1966) [20]. All results obtained during the analysis are stored in a binary memory and can be displayed or printed out.

\section{Discrimination in Point-Counting Systems}

To take full advantage of the merits of the point-count method, the discrimination devices should be adapted to the special needs.

Point-count methods have been used for stereometric analysis by K. Keil (1965) [32] and by P. Weinstein (1966) [23]. K. Keil used a manual control for the discrimination, but he pointed out that automation is easily possible. P. Weinstein adapted the concentration mapping device of K. F. J. Heinrich to decide whether or not the phase of interest is present.

It can be seen that the great advantage of the point-count technique lies in the rapid way of obtaining volume-fraction analyses, especially in the case of small amounts of the interesting phase and where only low counting rates are available. If the fixedcount method is used instead of a fixed-time measurement, it is 
much easier to preselect the maximum statistical error which can be tolerated to discriminate the phases present accurately. A further advantage of this method would be that the time needed for the total analysis is in many cases shorter than the time needed for lineal analysis.

The discriminator used in point-count analysis will work on a digital basis rather than on an analog one if $\mathrm{x}$-ray data are used. For the other signals (ref. section V.), analog discrimination might be preferable.

A measuring cycle should work approximately as outlined below. The electron beam is set to a "starting position" on phase 1 . The $\mathrm{x}-$ ray quanta of the selected elements for phase 1 are counted. If the selected number of counts is reached, counting is stopped and the time needed for the accumulation is processed into the analyzing circuits (discriminator or tape for computer analysis). After this, an "advance" signal is supplied to the step-scanning device. The electron beam moves to the next point and the counting procedure starts again. This should also be very useful for alloys with extremely varying concentrations of an element. When using lineal methods, the statistical accuracy will be much poorer in the lowconcentration regions than in the other ones.

\section{COUNTING AND SIZING CIRCUITS}

The pulse groups or the pulses, representing intercept length or point counts respectively, have to be stored, counted, or analyzed in units following the gate. The best way to discuss these units will probably be to divide them according to the stereometric parameters, which are analyzed thereby.

\section{Volume Fraction Analysis}

This is obviously the simplest way to obtain structural parameters. In lineal analysis only the fraction of the total time of analysis has to be determined, during which the electron beam remains within the selected phase (section VI.B.1.). Only one counter, which integrates all pulses passing the gate, is needed for this purpose.

In point-count analysis, the procedure is even simpler. If a phase is recognized, one pulse has to be stored in the corresponding counter. The ratio of the points falling within one selected phase tc 
the total number of points scanned during the analysis gives the areal or volume fraction of this phase.

\section{Surface Areas Per Unit Volume and Mean Diameter of a Phase}

As mentioned in section II.B., the surface-to-volume ratios very often show a clear correlation to physical properties of the investigated alloy. The measurement is also very simple. The number of intercepts analyzed has to be registered in a separate counter; from this number and from the length of the test line within the particular phase, the specific surface area $\left(\mathrm{S}_{v}\right)$ and the mean grainsize ( $\mathrm{L}$ ) of this phase can be calculated.

\section{Proximity (ref. section II.D.)}

The "sequence analyzer" proposed by G. Dörfler (1966) [14], analyzes the frequency of the sequence of three or more phases while scanning the surface along a line. The schematic diagram of the analyzing logic is given in Figure 12. One very important feature is that the simultaneous analysis of all phases present in a sample is possible. The working principle of the "sequence analyzer" can be described in the following way. If channel 1 is in function, it supplies a voltage to the bistable multivibrator (flip-flop $=F F$ ). This FF is thereby switched into its conductive state and now it supplies a voltage to the two AND-gates 12 and 13 . This is necessary because there is no evidence at this point whether the electron beam will pass from phase 1 into phase 2 or into phase 3 . Now, if the beam comes into phase 2, channel 2 starts and supplies a voltage to FF 2 and this, in turn, to the AND-gates 12 and 13. Channel 1 has stopped operation, but due to its electronic characteristics, the FF 1 still supplies the voltage to the AND-gates 12 and 13. By this way, only the AND-gate 12 obtains voltages at both branches and can now switch the counter 12 to the next number. The two remaining gates 13 and 23 are inactive because only one branch of each obtains a voltage. After the advance ( 1 number), the counter supplies a reset signal to the FF's 1 and 2; both are switched to the nonconductive state. Since channel 2 still supplies a voltage to FF 2, the same procedure starts again: FF 2 is transferred into the conductive state and the sequence analyzer is ready to register the next sequence. A voltage diagram is given in Figure 13.

Since only electronic devices are used, speed limitations lie mainly in the signal generators. 


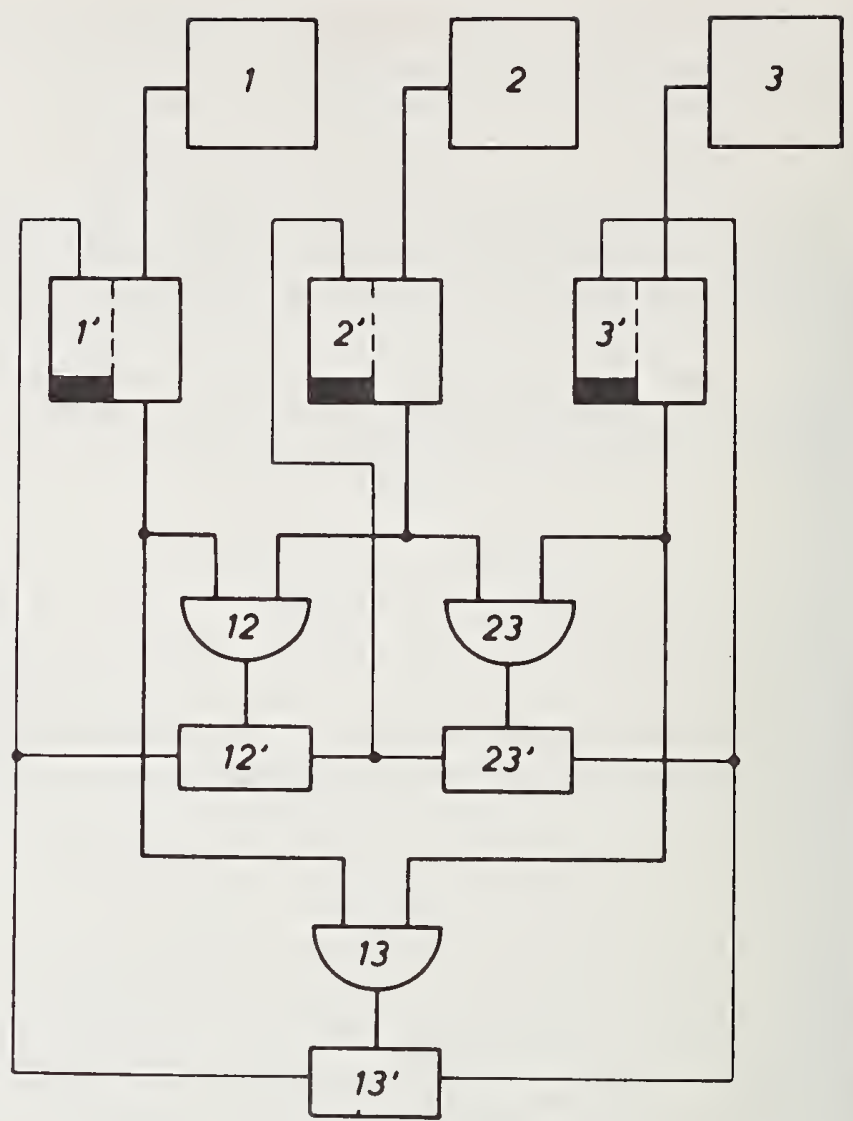

Figure 12. Arrangement of the "Sequence Analyzer": 1,2,3 = analyzing circuits $1^{\prime}, 2^{\prime}, 3^{\prime}=$ Flip-flops; 12, 13, $23=$ AND-gates; 12', 13', 23' = counters.

\section{Grainsize Distributions and Number of Particles Per Unit Volume}

These two stereometric parameters require the most sophisticated analyzing circuits, as can be seen from the "inclusion counter" of D A. Melford and K. R. Whittington $(1964,1965)$ [22,43] (ref. td section VI.B.2.).

According to the experience gained in light microscopy (H. E Exner, 1966) [18,19]; (G. Bockstiegel, 1966) [20], it seems to b. most expedient to obtain an "oversize distribution", which can b! 

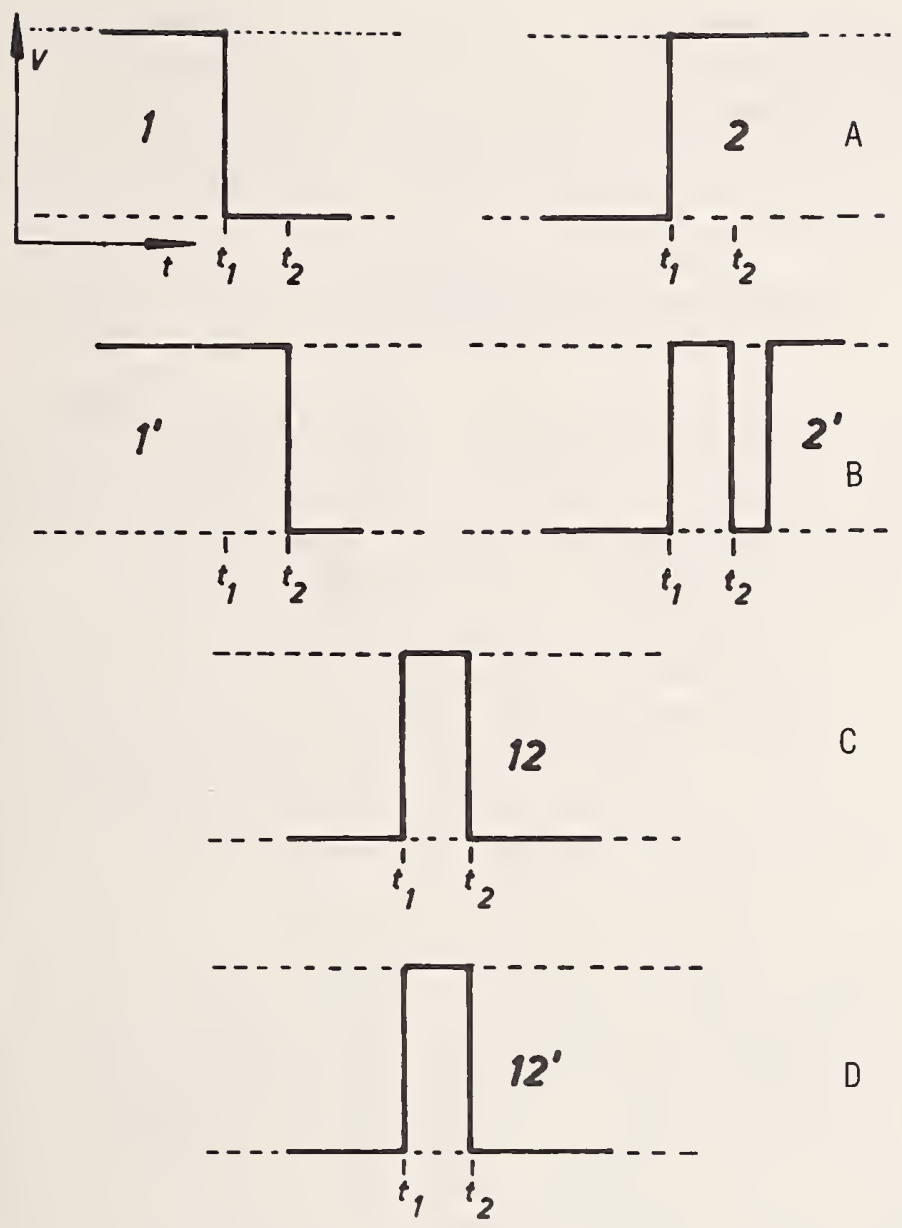

C

Figure 13. Voltage diagram, showing the principle of the "Sequence Analyzer".

converted to size distributions in space. The length intervals, which are covered by the size classes, have to be chosen according to the problem. Normally 1 to 15 classes are sufficient, which can be divided in a logarithmically-equidistant way (Exner, 1966) [18]. The intercept lengths are registered in the "integral method"; that means that all intercepts which are smaller than the upper limit of a given class are summed up in this class. This method has several 
considerable advantages. The comparison of several distributions is much easier than by the "histogram method", where the number of lengths falling within a class are counted separately.

From the same results, the number of particles per unit volume can also be determined (H. E. Exner, 1966) [18]; (G. Bockstiegel, 1966) [20].

\section{DATA REDUCTION AND ANALYSIS WITH A DIGITAL COMPUTER}

The most promising possibility seems to be the application of the digital computer for handling the great amount of data obtained during one analysis. A great deal of work has been done in the field of light microscopy, where the digital computer has been successfully applied by G. A. Moore $(1964,1966)[44,45]$.

Here some possibilities shall be discussed, which are presently under investigation in our laboratory. One great problem is the fact that our present analyzing systems are far too slow for a direct data transfer to the computer. Magnetic tape or paper tape has to be used as a storage device. It is very important that no information be lost during the storage and transfer process. One example may illustrate this. If all pulse groups (= intercept length) are integrated on a counter, information is no longer available concerning the other parameters such as grainsize, grainsize distribution, etc. The optimum would be to store all pulse groups for all phases without alteration of their relative position, their sequence and their position on subsequent lines. Under these circumstances, the most preferable device will be a magnetic-tape recorder, which can register relatively high pulse frequencies; this may be a multiple track recorder which is computer-compatible or, as in our laboratory, a normal tape recorder with some special modifications. Figure 14 shows the arrangement diagrammatically. The pulses of four channels are recorded on one track and are distinguished by their amplitudes. If all phases are analyzed simultaneously and the conditions are optimal, a continuous chain of pulses should exist, the amplitude of which changes with the sequence of the phases. The end of a scanning line can be indicated on a separate track to allow more sophisticated evaluations, which will be discussed at a later time. 


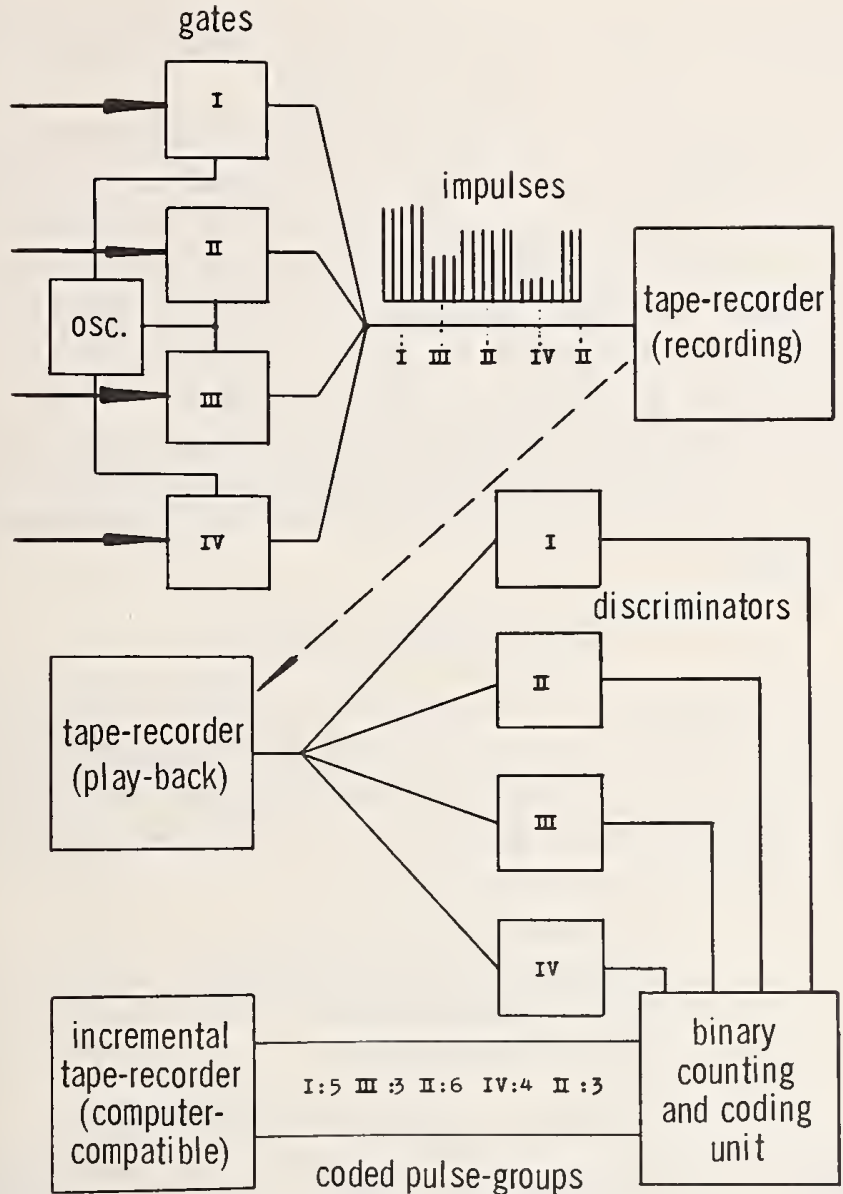

Figure 14. Arrangement for the computer evaluation of the data, supplied by the phase integrator.

The analysis can be recorded on the tape at relatively low speed while the playback may be much faster. Now the pulse groups of the individual phases have to be transferred onto magnetic tape; in this Way, a first data reduction can be accomplished: the individual pulse groups are sorted according to their amplitude by means of discriminator circuits (Figure 14) and are fed into counting circuits. The counting circuit of each phase adds the pulses of each pulse group and transfers it to the incremental tape recorder of the computer, simultaneously indicating the channel from which the 
pulse group came. Thus, one scanning line may perhaps look like $\mathrm{A}$ 16 , B 25, A 2, C 30, B 12, etc., (the letters mean phase type and numbers mean number of pulses (or microns).

If this tape is processed in the computer, applying an appropriate program, practically all stereometric parameters can be evaluated. The great advantage of this system will be that the computer can perform even very elaborate calculations involved in the determination of grainsize distributions of complex particles. Furthermore, error calculations, as they are described briefly in section VIII., can be performed more accurately.

The use of the digital computer for this purpose is a very new field and some experience should be gained. Certainly much of the work done by G. A. Moore $(1963 ; 1964 ; 1966)$ [44-47] can be applied to these purposes.

\section{Analyzing Conditions}

If the conditions of a stereometric analysis are discussed, a compromise between the maximum speed and the maximum error which can be tolerated has to be made. Therefore, it is necessary to discuss these two questions in one section. We have the following variables involved in the analysis:

the electron beam diameter

the time constant of the ratemeter

the threshold setting and

the scanning velocity

\section{A. INFLUENCE OF THE BEAM DIAMETER}

If there is a concentration discontinuity between two phases, the finite beam diameter will cause an observed concentration distribution, as illustrated in Figure 15. This function depends only on the geometry of the arrangement and not on the scanning velocity. Therefore, in stereometric analysis the beam diameter should be chosen as small as possible. More precisely it should be noted that an "x-ray emission diameter" rather than the beam diameter causes the above mentioned distribution curve.

\section{B. THE TIME CONSTANT OF THE RATEMETER AND THE PULSE- INTEGRATOR}

Both these digital-to-analog converters have been discussed in detail in section VI.A.; therefore, reference can be made to this section. 


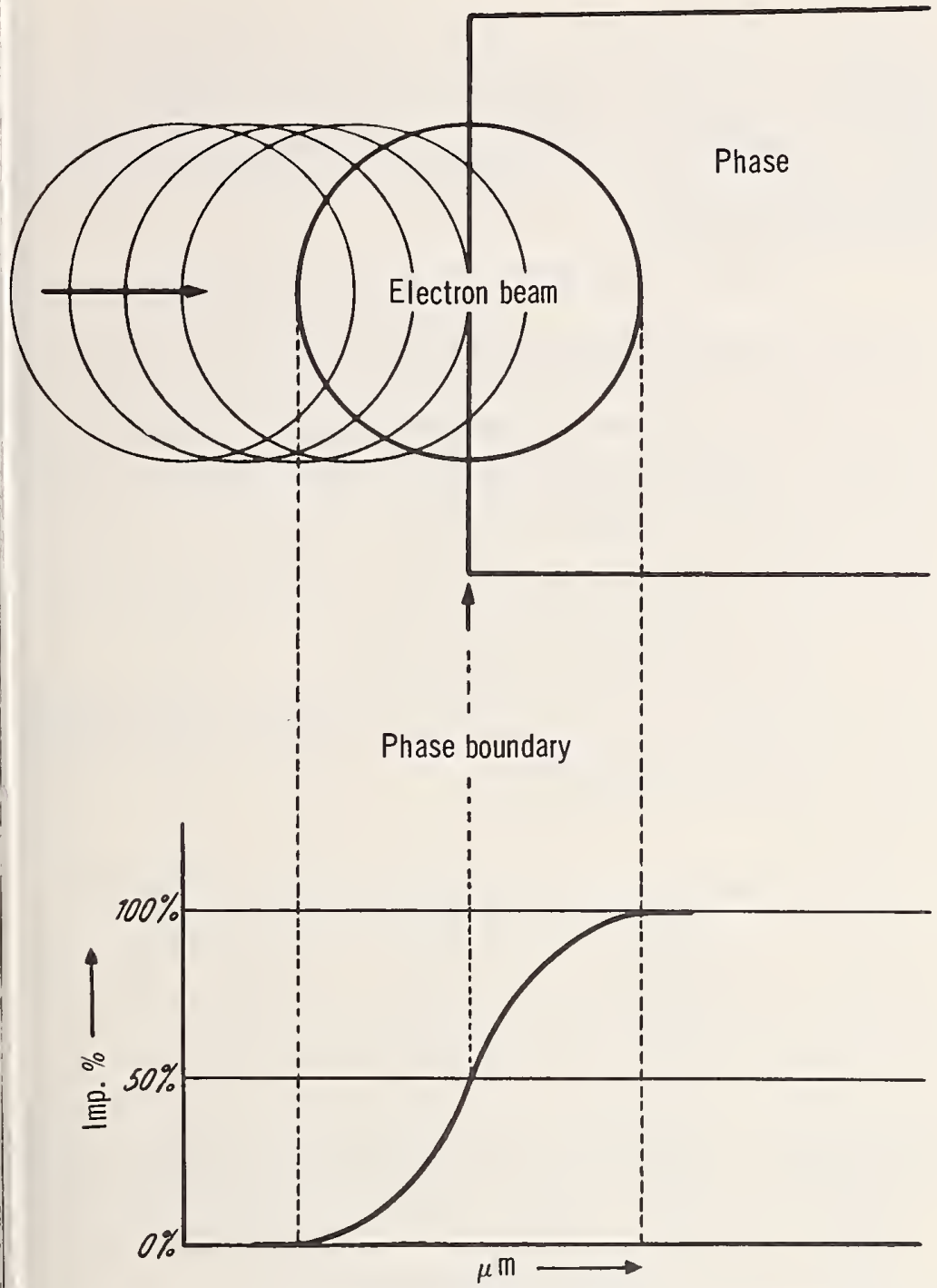

Figure 15. Influence of the finite beam diameter on the voltage supplied by the ratemeter (time constant is assumed to be negligible).

\section{INFLUENCE OF THE SCANNING VELOCITY}

Since the time constant of the ratemeter and the scanning velocity are tunctions of time, both are strongly interrelated. If a discontinuity in the concentration is assumed, the electron beam 
will travel some distance away from this discontinuity before the maximum value of the ratemeter output is obtained. For a quantitative length measurement, it is absolutely necessary that the effective signal value be reached before the electron beam leaves the phase again. Otherwise the threshold setting would depend on the grainsize of the phase.

\section{INFLUENCE OF THE THRESHOLD SETTING}

A simple consideration shows that the voltage change at the ratemeter must obey the same laws at the entrance into a phase and when leaving a phase. A "critical intercept length" can be calculated, at which the maximum value of the signal is reached just before the beam leaves the phase. Figure 16 shows several voltage changes for different conditions. The shape of the curve was obtained from the overlapping of the ratemeter- and the beamsize function. Oscillograms show that these assumptions are valid (K. F. J. Heinrich, private communication).

Due to the shape of this voltage curve it can be easily concluded that a correct discrimination can only be made at the $50 \%$ value between minimum and maximum.

\section{Count Rate (\%)}

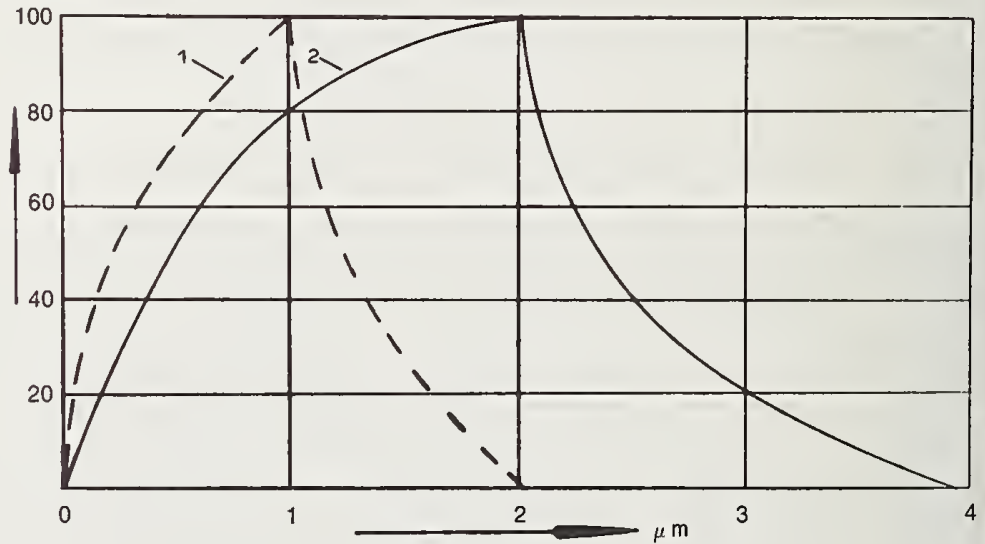

Figure 16. True voltage variation obtained by passing over a phase of critical size (the $100 \%$ value is just attained). 


\section{E. THE SCANNING VELOCITY}

This variable has already been included in Figure 16. It influences the spatial resolution of the measurement very strongly. A simple calculation shows that four times the time constant $(4 \sigma)$ has to elapse before the ratemeter voltage reaches $99 \%$ of the signal value. Therefore, the distance covered by the electron beam during $4 \sigma$ gives the spatial resolution of the method, provided that the electron beam is smaller than this size.

Figure 17 gives the interrelation between spatial resolution and scanning velocity plus beam size.

The application of the periodic pulse integrator as a signal source should give a considerable improvement in these cases.

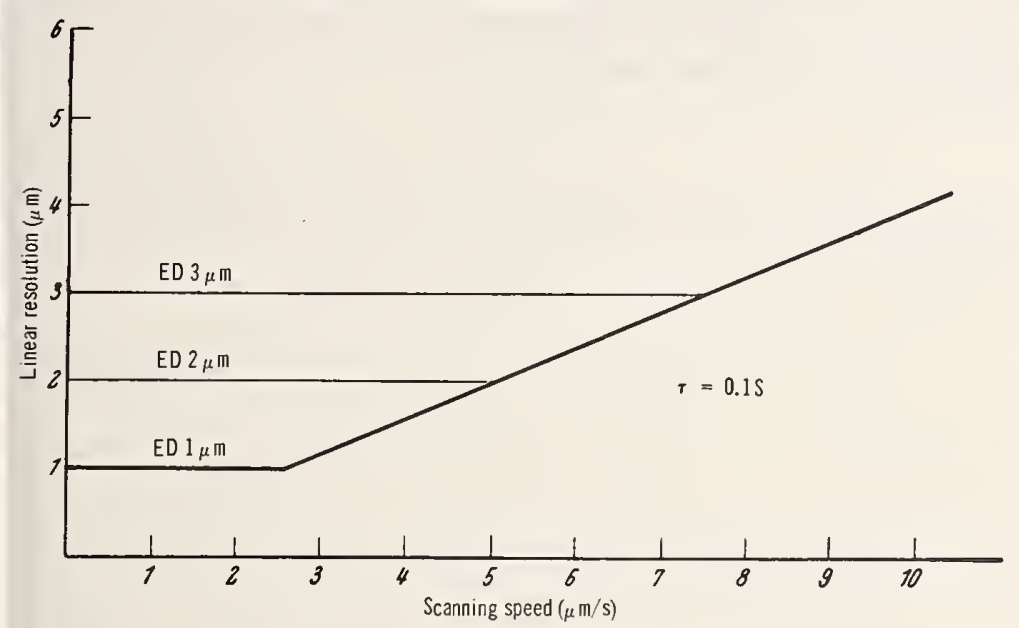

Figure 17. Lateral resolution obtained by scanning analysis ED = beam diameter; $\tau=$ time constant.

\section{Experimental Errors}

Errors in the length measurement can be involved in two ways. On one hand, the threshold settings may not be correct and the spatial resolution may not be sufficient; on the other hand, $\mathrm{x}$-ray státistics may not be good enough to allow an accurate identification of the phase. The errors caused by lack of resolution and poor statistics can be easily avoided and will not be treated here. 


\section{A. ERRORS DUE TO THE THRESHOLD SETTINGS}

If the settings of the thresholds are correct, no error should be involved in the analysis. However if more than two phases are present, it will be quite difficult to define where the $50 \%$ discrimination has to be set (e.g., the measured phase contains $50 \%$ of an element, the phases surrounding it contain $20 \%$ and $5 \%$ respectively).

Another effect which may cause errors in the threshold settings can be drift of the probe current - which could be avoided if the pulse rate/beam-current ratio is used.

For the following discussion, an error in the threshold setting of $50 \pm 10 \%$ ( $\pm 20 \%$ rel.) has been assumed. The deviation in microns from the true value has been measured on Figure 16.

Figure 18 gives the relative error depending on the experimental conditions and the mean grainsize.

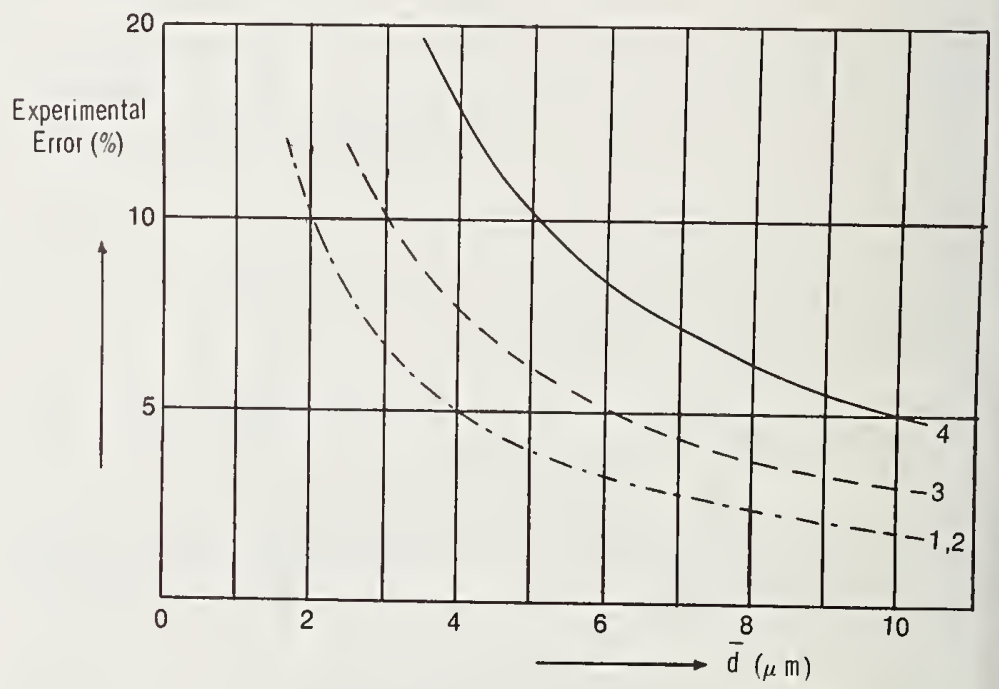

Figure 18. Experimental error in the length measurement as a function of mea grainsize (d) and the analyzing conditions:

$$
\begin{aligned}
& 1=\operatorname{scan} \quad 1 \mu / \mathrm{s} ; \text { beam }=1 \mu, \tau=0.1 \mathrm{~s} \\
& 2=\operatorname{scan} 5 \mu / \mathrm{s} ; \text { beam }=1 \mu, \tau=0.1 \mathrm{~s} \\
& 3=\operatorname{scan} 10 \mu / \mathrm{s} ; \text { beam }=1 \mu, \tau=0.1 \mathrm{~s} \\
& 4=\operatorname{scan} 8.3 \mu / \mathrm{s} ; \text { beam }=3 \mu, \tau=0.1 \mathrm{~s}
\end{aligned}
$$


B. STATISTICAL ERRORS IN THE ESTIMATION OF VOLUME COMPOSITION FROM DATA OBTAINED ON A SURFACE

These errors have been treated very extensively by S. A. Saltykov (1958) [9] and can be discussed here only very briefly.

It has been shown by the aforementioned author that the expected standard deviation can only be evaluated experimentally. It depends on the areal fraction of the phase analyzed, the number of intersections measured, and a shape coefficient.

The tables 3 and 4 give the absolute errors of the volume fraction analysis for two confidence limits (50\% and $95.4 \%$ respectively). Both have been calculated by Saltykov for a shape coefficient $K=1$.

This, however, is the most unlikely possibility. For an isotropic distribution, $\mathrm{K}=0.65$; for an oriented distribution with measurements normal to the orientation, $K=0.34$. According to the formula given in Tables 3-4, the measured number of intercepts has to be divided by $\mathrm{K}^{2}$ to give the corrected number of intersections used in Tables 3 and 4.

In Figure 19, the dependence of the statistical error on the number of intersections and the areal fraction of the phase is shown.

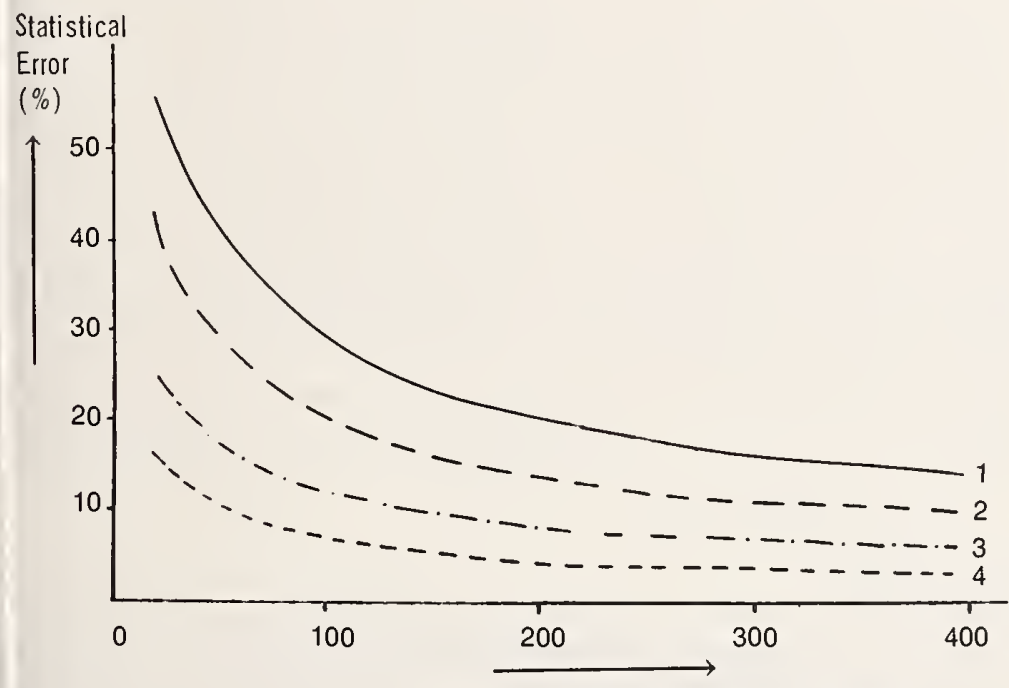

Number of Intercepts (counts)

Figure 19. Statistical error, introduced by the conclusion from the surface to the volume. 


\section{ELECTRON PRORE}

Table 3

Volume $\%$ of phase

\begin{tabular}{|c|c|c|c|c|c|c|c|c|c|c|c|c|c|c|}
\hline A & $\begin{array}{r}1 \\
99\end{array}$ & $\begin{array}{r}2 \\
98\end{array}$ & $\begin{array}{r}3 \\
97\end{array}$ & $\begin{array}{r}4 \\
96\end{array}$ & $\begin{array}{r}5 \\
95\end{array}$ & $\begin{array}{l}10 \\
90\end{array}$ & $\begin{array}{l}15 \\
85\end{array}$ & $\begin{array}{l}20 \\
80\end{array}$ & $\begin{array}{l}25 \\
75\end{array}$ & $\begin{array}{l}30 \\
70\end{array}$ & $\begin{array}{l}35 \\
65\end{array}$ & $\begin{array}{l}40 \\
60\end{array}$ & $\begin{array}{l}45 \\
55\end{array}$ & 50 \\
\hline 10 & 2.10 & 2.96 & 3.60 & 4.14 & 4.60 & 6.33 & 7.53 & 8.44 & 9.13 & 9.65 & 10.05 & 10.31 & 10.48 & 10.52 \\
\hline 20 & 1.48 & 2.08 & 2.54 & 2.92 & 3.25 & 4.47 & 5.32 & 5.96 & 6.46 & 6.83 & 7.11 & 7.30 & 7.41 & 7.45 \\
\hline 50 & 0.94 & 1.32 & 1.61 & 1.85 & 2.06 & 2.83 & 3.36 & 3.77 & 4.08 & 4.32 & 4.49 & 4.61 & 4.69 & 4.71 \\
\hline 100 & 0.66 & 0.93 & 1.14 & 1.31 & 1.45 & 2.00 & 2.38 & 2.67 & 2.89 & 3.06 & 3.18 & 3.27 & 3.32 & 3.33 \\
\hline 200 & 0.47 & 0.66 & 0.80 & 0.92 & 1.03 & 1.41 & 1.68 & 1.88 & 2.04 & 2.16 & 2.25 & 2.31 & 2.34 & 2.36 \\
\hline 300 & 0.38 & 0.54 & 0.66 & 0.75 & 0.84 & 1.15 & 1.37 & 1.54 & 1.67 & 1.76 & 1.83 & 1.88 & $1: 91$ & 1.92 \\
\hline 400 & 0.33 & 0.47 & 0.57 & 0.65 & 0.73 & 1.00 & 1.19 & 1.33 & 1.44 & 1.53 & 1.59 & 1.63 & 1.66 & 1.67 \\
\hline 500 & 0.30 & 0.42 & 0.51 & 0.58 & 0.65 & 0.89 & 1.06 & 1.19 & 1.29 & 1.36 & 1.42 & 1.46 & 1.48 & 1.49 \\
\hline 600 & 0.27 & 0.38 & 0.46 & 0.53 & 0.59 & 0.82 & 0.97 & 1.09 & 1.18 & 1.25 & 1.30 & 1.33 & 1.35 & 1.36 \\
\hline 700 & 0.25 & 0.35 & 0.43 & 0.49 & 0.55 & 0.76 & 0.90 & 1.01 & 1.09 & 1.15 & 1.20 & 1.23 & 1.25 & 1.26 \\
\hline 800 & 0.23 & 0.33 & 0.40 & 0.46 & 0.51 & 0.71 & 0.84 & 0.94 & 1.02 & 1.08 & 1.12 & 1.15 & 1.17 & 1.18 \\
\hline 900 & 0.22 & 0.31 & 0.38 & 0.44 & 0.48 & 0.67 & 0.79 & 0.89 & 0.96 & 1.02 & 1.06 & 1.09 & 1.11 & 1.11 \\
\hline 1000 & 0.21 & 0.30 & 0.36 & 0.41 & 0.46 & 0.63 & 0.75 & 0.81 & 0.91 & 0.97 & 1.00 & 1.03 & 1.05 & 1.05 \\
\hline 2000 & 0.15 & 0.21 & 0.25 & 0.29 & 0.32 & 0.45 & 0.53 & 0.60 & 0.65 & 0.68 & 0.71 & 0.73 & 0.74 & 0.74 \\
\hline 3000 & 0.12 & 0.17 & 0.21 & 0.24 & 0.27 & 0.37 & 0.43 & 0.49 & 0.53 & 0.56 & 0.58 & 0.60 & 0.61 & 0.61 \\
\hline 4000 & 0.10 & 0.15 & 0.18 & 0.21 & 0.23 & 0.32 & 0.38 & 0.42 & 0.46 & 0.48 & 0.50 & 0.52 & 0.52 & 0.53 \\
\hline 5000 & 0.09 & 0.13 & 0.16 & 0.18 & 0.21 & 0.28 & 0.34 & 0.38 & 0.41 & 0.43 & 0.45 & 0.46 & 0.47 & 0.47 \\
\hline 10000 & 0.07 & 0.09 & 0.11 & 0.13 & 0.15 & 0.20 & 024 & 0.27 & 0.29 & 0.31 & 0.32 & 0.33 & 0.33 & 0.33 \\
\hline 15000 & 0.05 & 0.08 & 0.09 & 0.11 & 0.12 & 0.16 & 0.19 & 0.22 & 0.24 & 0.25 & 0.26 & 0.27 & 0.27 & 0.27 \\
\hline 20000 & 0.05 & 0.07 & 0.08 & 0.09 & 0.10 & 0.14 & 0.17 & 0.19 & 0.20 & 0.22 & 0.22 & 0.23 & 0.23 & 0.24 \\
\hline 25000 & 0.04 & 0.06 & 0.07 & 0.08 & 0.09 & 0.13 & 0.15 & 0.17 & 0.18 & 0.19 & 0.20 & 0.21 & 0.21 & 0.21 \\
\hline 30000 & 0.04 & 0.05 & 0.07 & 0.08 & 0.08 & 0.12 & 0.14 & 0.15 & 0.17 & 0.18 & 0.18 & 0.19 & 0.19 & 0.19 \\
\hline 40000 & 0.03 & 0.05 & 0.06 & 0.07 & 0.07 & 0.10 & 0.12 & 0.13 & 0.14 & 0.15 & 0.16 & 0.16 & 0.17 & 0.17 \\
\hline 50000 & 0.03 & 0.04 & 0.05 & 0.06 & 0.06 & 0.09 & 0.11 & 0.12 & 0.13 & 0.14 & 0.14 & 0.15 & 0.15 & 0.15 \\
\hline 100000 & 0.02 & 0.03 & 0.04 & 0.04 & 0.05 & 0.06 & 0.08 & 0.08 & 0.09 & 0.10 & 0.10 & 0.10 & 0.10 & 0.11 \\
\hline & & & & & & & & & & & & & & \\
\hline
\end{tabular}

C. THE TOTAL EXPERIMENTAL ERROR

The total error can be calculated easily from the sources mentioned in VIII.A. and VIII.B., assuming a normal erro propagation ( $\mathrm{f}^{2}$ app $+\mathrm{f}^{2}$ stat $=\mathrm{f}^{2}$ total). This dependence is given ii 
Table 4

Volume $\%$ of Phase

\begin{tabular}{|c|c|c|c|c|c|c|c|c|c|c|c|c|c|c|c|}
\hline A & $\begin{array}{r}0 \\
100\end{array}$ & $\begin{array}{c}1 \\
99\end{array}$ & $\begin{array}{r}2 \\
98\end{array}$ & $\begin{array}{r}3 \\
97\end{array}$ & $\begin{array}{r}4 \\
96\end{array}$ & $\begin{array}{r}5 \\
95\end{array}$ & $\begin{array}{l}10 \\
90\end{array}$ & $\begin{array}{l}15 \\
85\end{array}$ & $\begin{array}{l}20 \\
80\end{array}$ & $\begin{array}{l}25 \\
75\end{array}$ & $\begin{array}{l}30 \\
70\end{array}$ & $\begin{array}{l}35 \\
65\end{array}$ & $\begin{array}{l}40 \\
60\end{array}$ & $\begin{array}{l}45 \\
55\end{array}$ & 50 \\
\hline 50 & - & - & - & - & - & - & - & - & - & - & - & - & 13.83 & 14.07 & 14.13 \\
\hline 100 & - & - & - & - & - & - & - & 7.14 & 8.00 & 8.67 & 9.18 & 9.54 & 9.81 & 9.96 & 10.00 \\
\hline 200 & - & - & - & - & - & - & 4.23 & 5.04 & 5.64 & 6.12 & 6.48 & 6.75 & 6.93 & 7.02 & 7.08 \\
\hline 300 & - & - & - & - & - & 2.52 & 3.45 & 4.11 & 4.62 & 5.00 & 5.28 & 5.49 & 5.64 & 5.73 & 5.76 \\
\hline 400 & - & - & - & - & 1.95 & 2.19 & 3.00 & 3.57 & 4.00 & 4.32 & 4.59 & 4.77 & 4.89 & 4.98 & 5.00 \\
\hline 500 & - & - & - & 1.53 & 1.74 & 1.95 & 2.67 & 3.18 & 3.57 & 3.87 & 4.08 & 4.26 & 4.38 & 4.44 & 4.47 \\
\hline 600 & - & - & 1.14 & 1.38 & 1.59 & 1.77 & 2.46 & 2.91 & 3.27 & 3.54 & 3.75 & 3.90 & 4.00 & 4.05 & 4.08 \\
\hline 700 & - & - & 1.05 & 1.29 & 1.47 & 1.65 & 2.28 & 2.70 & 3.03 & 3.27 & 3.45 & 3.60 & 3.69 & 3.75 & 3.78 \\
\hline 800 & - & - & 1.00 & 1.20 & 1.38 & 1.53 & 2.13 & 2.52 & 2.82 & 3.06 & 3.24 & 3.36 & 3.45 & 3.51 & 354 \\
\hline 900 & - & - & 0.93 & 1.14 & 1.32 & 1.44 & 2.00 & 2.37 & 2.67 & 2.88 & 3.06 & 3.18 & 3.27 & 3.33 & \\
\hline 1000 & - & - & 0.90 & 1.08 & 1.23 & 1.38 & 1.89 & 2.25 & 2.52 & 2.73 & 2.91 & 3.00 & 3.09 & 3.15 & \\
\hline 2000 & - & 0.45 & 0.63 & 0.75 & 0.87 & 0.96 & 1.35 & 1.59 & 1.80 & 1.95 & 2.04 & 2.13 & 2.19 & 2.22 & 2.22 \\
\hline 3000 & - & 0.36 & 0.51 & 0.63 . & 0.72 & 0.81 & 1.11 & 1.29 & 1.47 & 1.59 & 1.68 & 1.74 & 1.80 & 1.83 & 1.83 \\
\hline 4000 & - & 0.30 & 0.45 & 0.54 & 0.63 & 0.69 & 0.96 & 1.14 & 1.26 & 1.38 & 1.44 & 1.50 & 1.56 & 1.56 & 1.59 \\
\hline 5000 & - & 0.27 & 0.39 & 0.48 & 0.54 & 0.63 & 0.84 & 1.02 & 1.14 & 1.23 & 1.29 & 1.35 & 1.38 & 1.41 & 1.41 \\
\hline 10000 & - & 0.21 & 0.27 & 0.33 & 0.39 & 0.45 & 0.60 & 0.72 & 0.81 & 0.87 & 0.93 & 0.96 & 0.99 & 1.00 & 1.00 \\
\hline 15000 & - & 0.15 & 0.24 & 0.27 & 0.33 & 0.36 & 0.48 & 0.57 & 0.66 & 0.72 & 0.75 & 0.78 & 0.80 & 0.81 & 0.81 \\
\hline 20000 & - & 0.15 & 0.21 & 0.24 & 0.27 & 0.30 & 0.42 & 0.51 & 0.57 & 0.60 & 0.66 & 0.66 & 0.69 & 0.69 & 0.72 \\
\hline 25000 & - & 0.12 & 0.18 & 0.21 & 0.24 & 0.27 & 0.39 & 0.45 & 0.51 & 0.54 & 0.57 & 0.60 & 0.63 & 0.63 & 0.63 \\
\hline 30000 & - & 0.12 & 0.15 & 0.21 & 0.24 & 0.24 & 0.36 & 0.42 & 0.45 & 0.51 & 0.54 & 0.54 & 0.57 & 0.57 & 0.57 \\
\hline 40000 & - & 0.09 & 0.15 & 0.18 & 0.21 & 0.21 & 0.30 & 0.36 & 0.39 & 0.42 & 0.45 & 0.48 & 0.48 & 0.51 & 0.51 \\
\hline 50000 & - & 0.09 & 0.12 & 0.15 & 0.18 & 0.18 & 0.27 & 0.33 & 0.36 & 0.39 & 0.42 & 0.42 & 0.45 & 0.45 & 0.45 \\
\hline 00000 & - & 0.06 & 0.09 & 0.12 & 0.12 & 0.15 & 0.18 & 0.24 & 0.24 & 0.27 & 0.30 & 0.30 & 0.30 & 0.31 & 0.33 \\
\hline
\end{tabular}

Yeliability $=95.44 \%$

Figure 20. From the Figure it can be concluded that a low experimental error can be achieved only if the error of the analysis is well as the statistical error is small. Therefore, an accumulation of a great many data points (intersections, point counts) is in most ases far more important than a very small error caused by the ipparatus. 


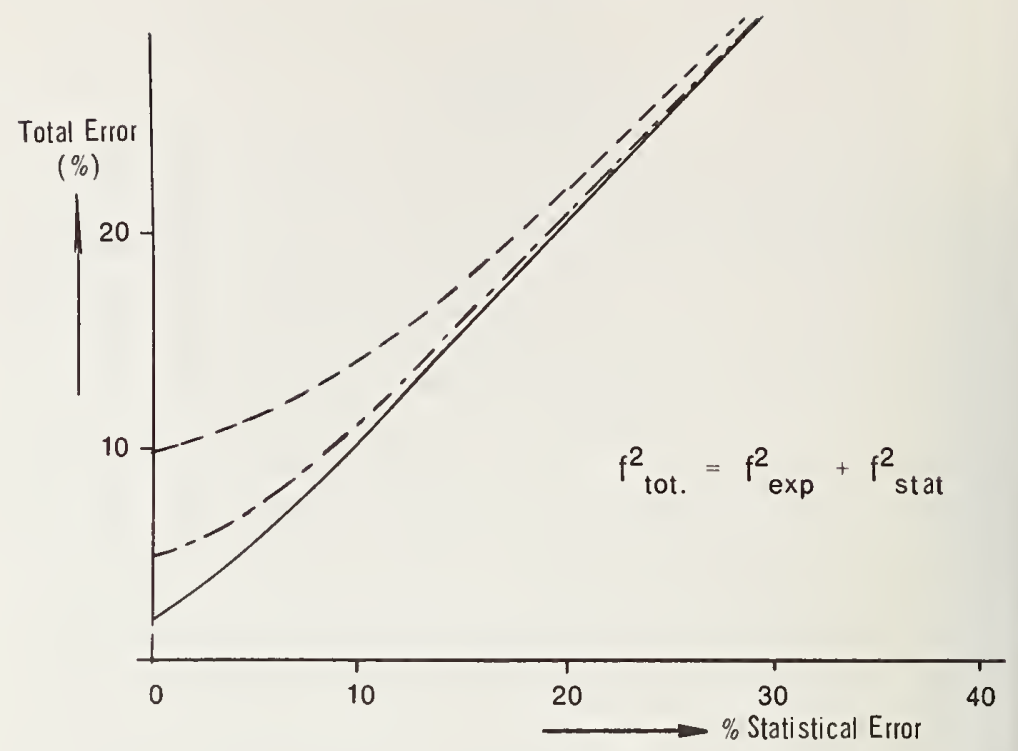

Figure 20. Total error of the analysis.

\section{Applications (Examples and further possibilities)}

Because of the short time during which the instruments discussed have been available, all the application possibilities have not been completely explored (not very many applications have been published). In this section it shall be mainly pointed out where the applications of the microprobe as a signal generator seem to be useful and where very interesting results may be expected. In the majority of the already published examples, the volume fractions of phases have been of primary interest.

\section{A. VOLUME FRACTION ANALYSIS}

K. Keil (1965) [32] used the point-count method (ref. sectior VI.B.3.) to perform a mineralogical modal analysis (= stereometrif analysis) on meteorite specimens.

J. M. Ramsey and P. Weinstein (1966) [43] performed a linea analysis by means of the instrument mentioned in section III of $10 / 90$ tin-lead samples with various gold additions. The areas user for the analysis were $340 \mu$ square and three areas were analyzed of each sample. The results of these measurements are given in Figur 21. 


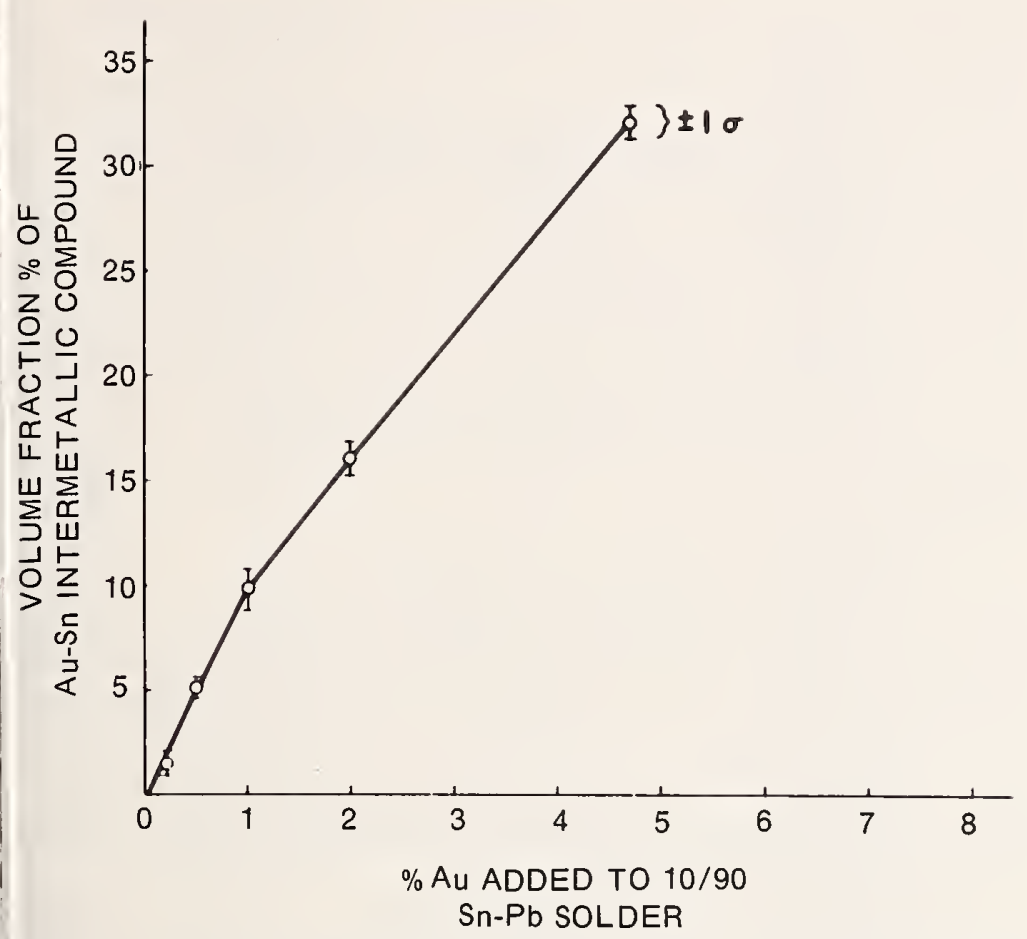

Figure 21. Example of lineal analysis by the electron probe (Ramsey and Weinstein, 1966).

Several results have been published by G. Dörfler $(1965 ; 1966$; 1967) $[14,15,24,49-52]$. Some interesting results shall be discussed here.

A very important and sometimes difficult problem is the determination of the ferrite and austenite volume fractions in high grade steels. Since the ferrite content strongly influences the resistance against corrosion and the mechanical properties, an accurate method for the determination would be advantageous.

In light-microscopy two major problems arise. On the one hand, manual integration methods are by far too tedious to be generally applicable; on the other hand, automation by means of a television microscope is nearly impossible because of the faint contrast between ferrite and austenite, if normal etchants are applied. The reliability of the results by other physical methods for this 
determination (e.g., the magnetic balance and $\mathrm{x}$-ray diffraction methods) suffer from the influence of inhomogeneities. However, if the electron probe and the phase integrator are used (G. Dörfler, $1965 ; 1966)$, ferrite and austenite can be discriminated by means of their different Ni-contents (in high grade steels), and influences of inhomogeneities can be excluded up to a certain degree. Table 5 gives the results of a considerable number of analyses which have been compared with light microscopy. The indicated errors have been calculated according to the procedures discussed in section VIII. The standard deviation is much lower if repetitive measurements on the same area are performed; however, in practice, only the total error of the volume-fraction analysis is of interest.

Two problems have been investigated in iron meteorites concerning some interrelations between phosphides and the Widmanstätten structure. Measurements of the volume fraction of kamacite ( $\alpha$-phase), taenite ( $\mu$-phase), and schreibersite (phosphide) have been performed in a great number of so-called plessite fields. Figure 22 gives the relationship which has been found. The results show a clear increase of $\alpha$-phase with increasing phosphide content (G. Dörfler, 1966; 1967) [14,52].

In the latter investigation (G. Dörfler, 1966) [14] another possible application of stereometric techniques is shown. In most kinetic considerations, the distribution of the various phases is of great interest. Here, the phosphide distribution relative to the Widmanstätten structure has been determined. The distance between two taenite-lamellae in the kamacite has been divided into $100 \%$ (Figure 23a) and a great number of such structures have been scanned in small increments, with the volume fraction of phosphides being measured in each increment. The result of all measurements is given in Figure $23 \mathrm{~b}$, and it is clearly shown that a grain-boundary nucleation of the phosphides takes place.

Perhaps one of the most promising possibilities of the electron microprobe in stereometric analysis lies in the quantitative structural measurements on segregated materials. In this field practically no other methods are applicable, while the microprobe permits the collection of very accurate results. J. Philibert and his group (J. Philibert et al. 1959) [53]; (C. Crussard et al. 1959) [54] developed a method for characterizing segregations of certair elements in steel by the ratio of the maximum to the minimum to the 


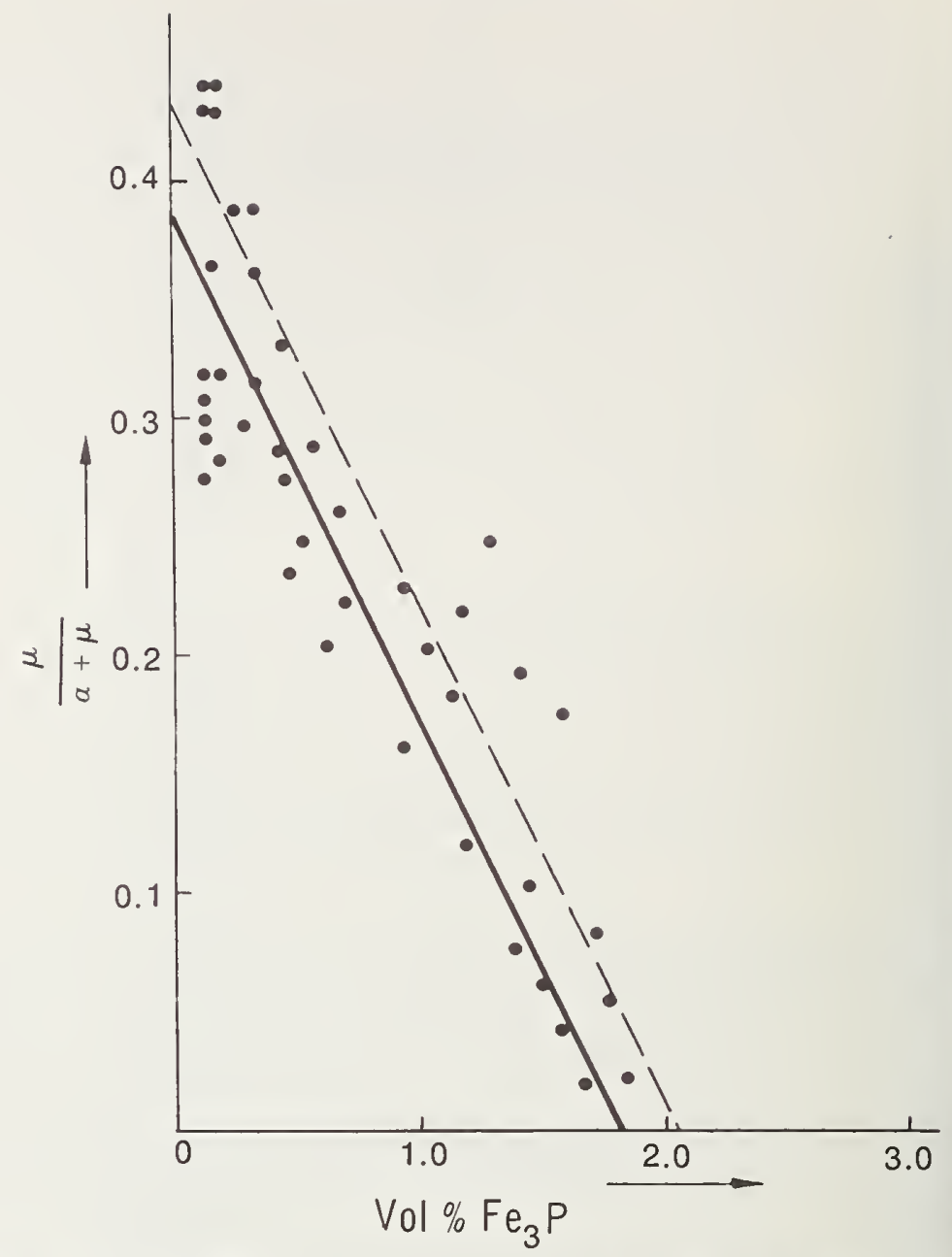

Figure 22. Dependence of the $\mu /(\alpha+\mu)$ ratio on the amount of phosphides in metallic meteorites (Dorfler, 1967) [52].

concentration of the encountered element and by the mean distance of the segregations. However, not only these parameters but also the frequency of segregation peaks and minima have certainly a great influence on the physical properties of a material. G. Dörfler, E. Plöckinger, and K. Swoboda (1966) [15] investigated the annealing kinetics of an Fe-Ni-P alloy containing $12 \% \mathrm{Ni}$ and $0.5 \% \mathrm{P}$. The segregations observed in a line scan can be seen in Figure 24. Four 


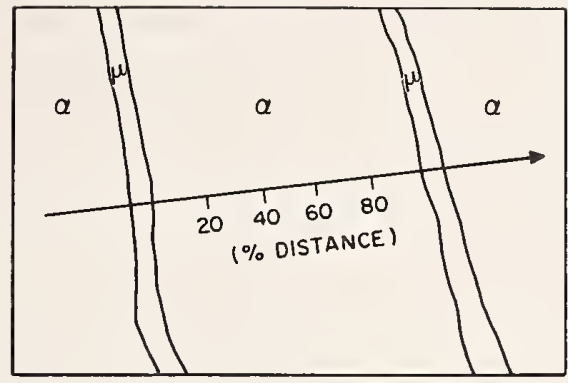

(o)

$\% \mathrm{Fe}_{3} \mathrm{P}$

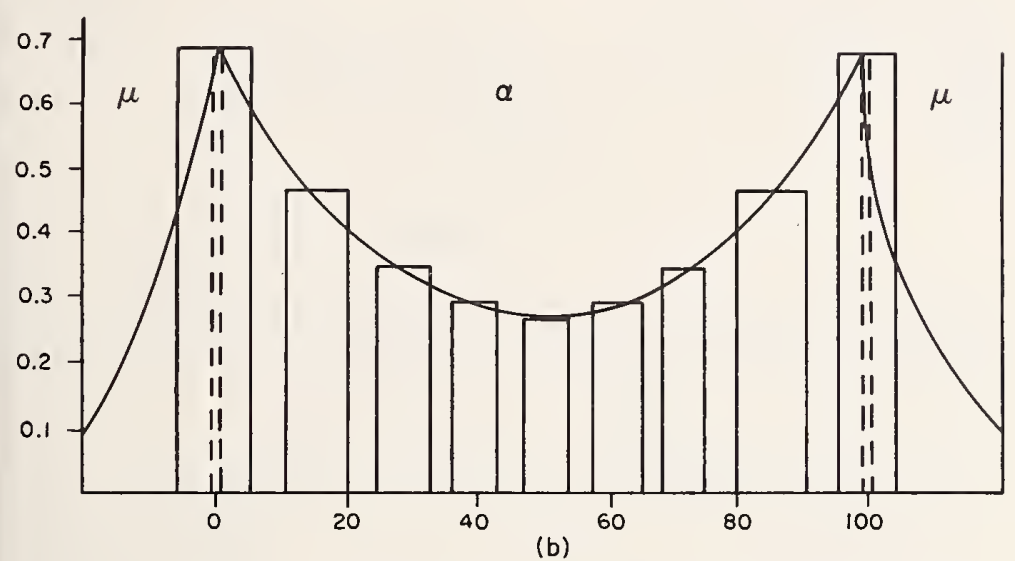

Figure 23. Quantitative determination of the phosphide distribution between $\mu$ plates in iron meteorites; 23a: schematic sketch how to divide the distance between two $\mu$-plates into $100 \%, 23 \mathrm{~b}$ : distribution of phosphides between $\mu$ plates.

concentration ranges (I-IV) have been selected by the threshold settings, and the volume fractions of the alloy falling within these limits have been measured.

Since annealing at $1300{ }^{\circ} \mathrm{C}$ should bring the alloy into the onephase field, the P-concentration of the whole alloy should lie within the limits of range II $(0.33-0.66 \% \mathrm{P})$, thus giving $100 \%$ (vol). Figure 25 shows the results for increasing annealing times. The measurement gives quite a good indication of the kinetic reaction process. 


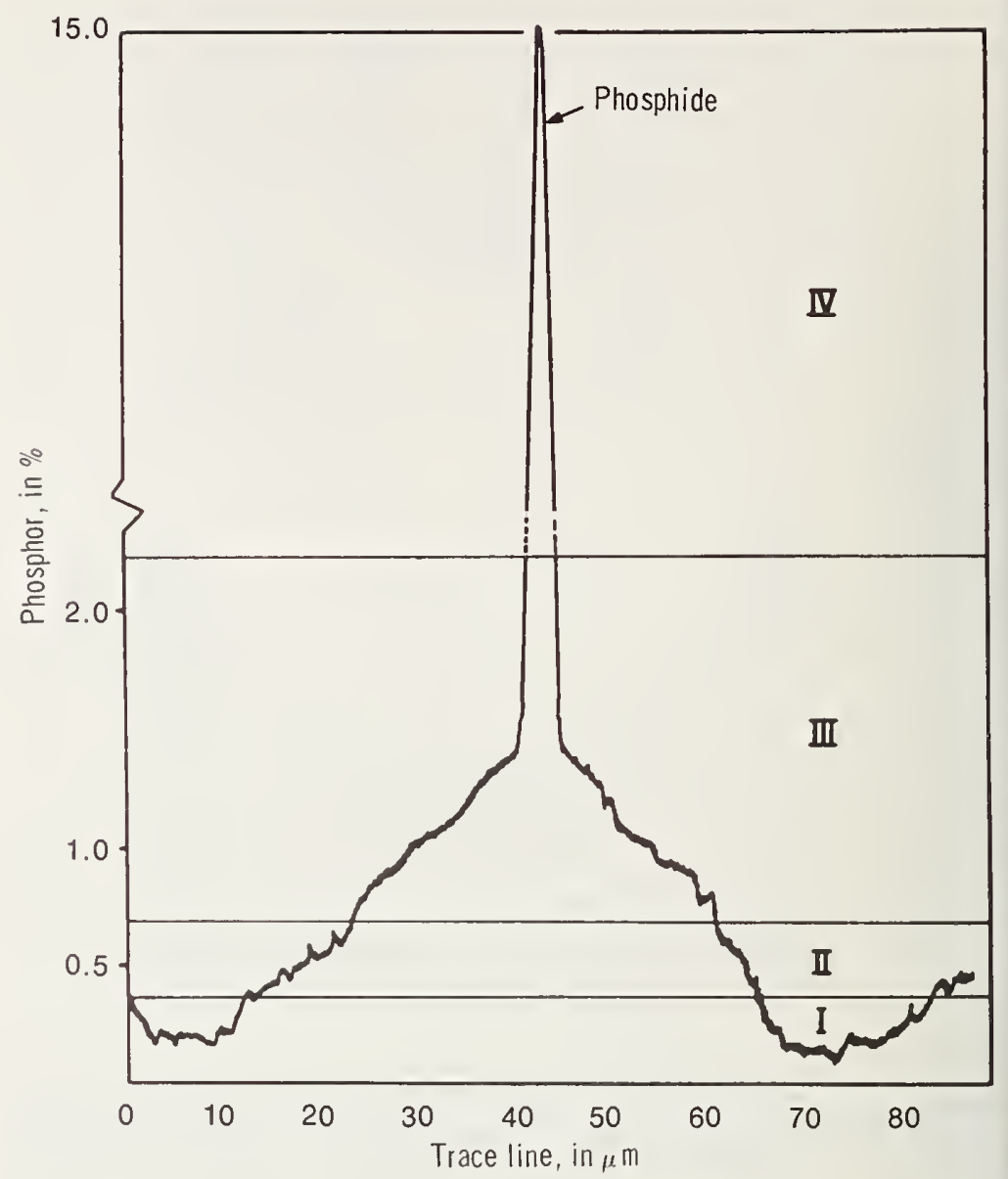

Figure 24. Phosphorus segregation in an as-cast Fe-Ni-P alloy (12\% Ni, 0.5\% P):

I: Channel I ( $<0.33 \%$ P); II: Channel II (0.33-0.66\% P); III: Channel III (0.66$2.22 \%$ P); IV: Channel IV ( $>2.22 \%$ P), for the "Phase Integrator".

\section{B. DETERMINATION OF THE MEAN DIAMETER OF PHASES}

Measurements of this parameter have been made by $\mathrm{R}$. Theisen (1964) [21]. He investigated the structural characteristics of $\mathrm{FeAl}_{3}$ inclusions in SAP (sintered aluminium powder). The spectrometer was set to the FeK radiation and the sample was scanned mechanically $100 \times 100 \mu^{2}$. During the scan, all Fe impulses were recorded; a modified recorder equipped with an electro-mechanical 


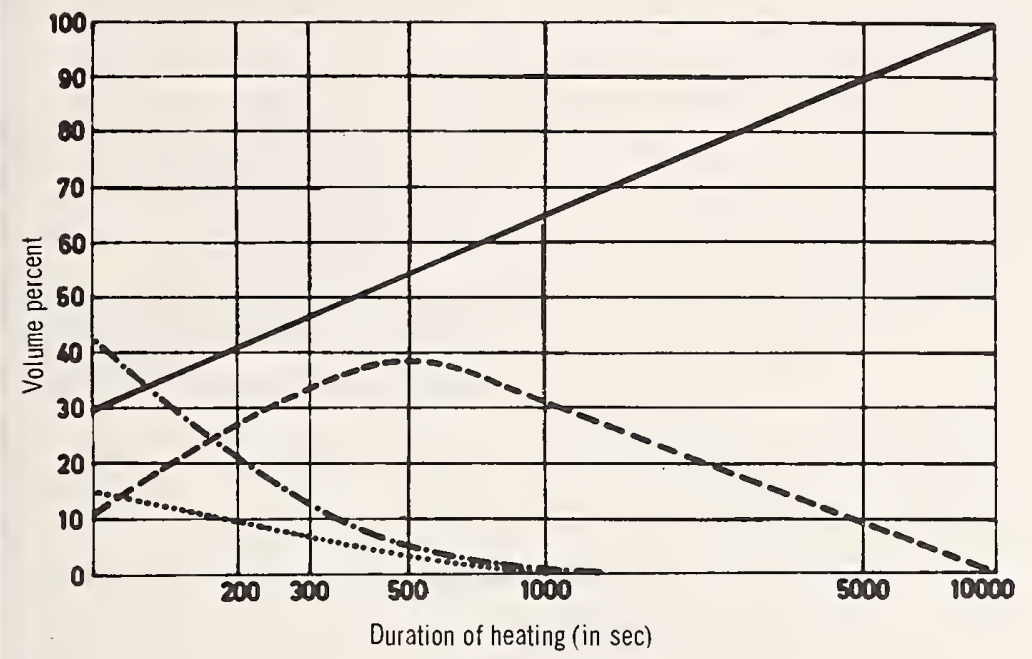

Figure 25. Annealing kinetics of the alloy mentioned in Fig. 24.

counter registered the number of particles intersected. The mean diameter of the $\mathrm{FeAl}_{3}$ particles can be calculated from the formula:

$\bar{d}=\frac{x^{4}}{n}$

$x=\frac{N_{(\mathrm{Fe})}-10^{4} \mathrm{U}}{\mathrm{N}_{(\mathrm{Fe})}^{\prime}-\mathrm{U}}$

$$
\mathrm{n}=\text { number of intersections }
$$

$\mathrm{N}_{(\mathrm{Fe})}=$ number of counts on Fe K during 20 minutes on pure iron (scanning $10^{4}$ microns)

$$
\begin{aligned}
\mathrm{N}_{(\mathrm{Fe})}^{\prime}= & \text { number of counts on Fe K } \\
& \text { during } 0.12 \mathrm{sec}(=1 \mu \text { at that } \\
& \text { scanning velocity }) \\
\mathrm{U}= & \text { background counts on Fe K } \\
& \text { during } 0.12 \text { seconds, measured } \\
& \text { on SAP }
\end{aligned}
$$


The mass-concentration of the inclusions can be determined by the formula:

$\mathrm{C}_{E}=\frac{\mathrm{C}_{1}}{\mathrm{C}_{2}}$

$\mathrm{C}_{1}=$ ratio of counts on $\mathrm{Fe} \mathrm{K}$ between pure iron and SAP surface while scanning $10^{4}$ microns

$\mathrm{C}_{2}=$ ratio of the intensities on $\mathrm{Fe} \mathrm{K}$ between pure iron and $\mathrm{FeAl}_{3}$, measured with a static probe

The results of the experiments are given in Figure 26.
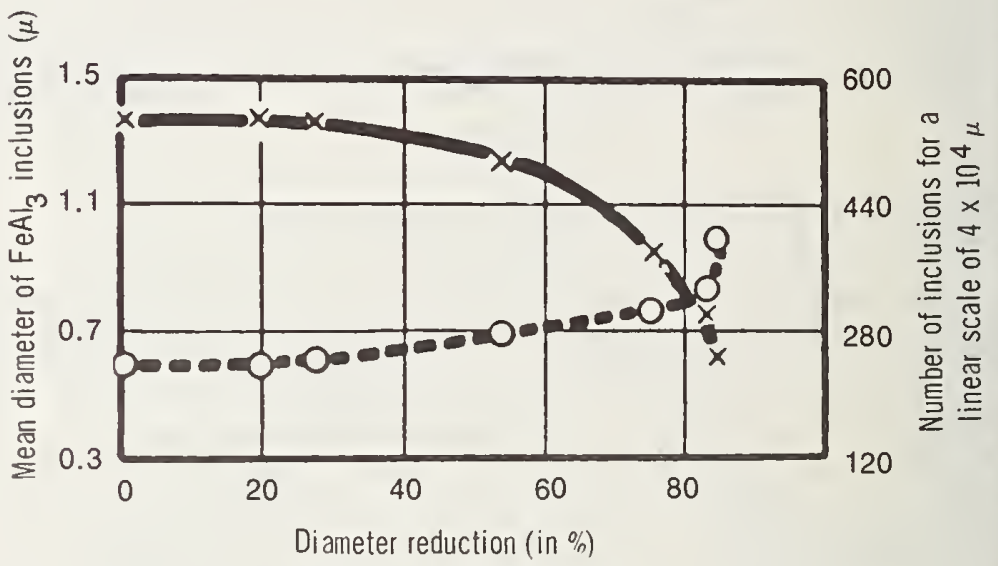

Figure 26. Influence of degree of deformation on mean diameter and number of $\mathrm{FeAl}_{3}$ inclusions in SAP-ISML $\left(7 \% \mathrm{Al}_{2} \mathrm{O}_{3}\right.$ ) (Theisen, 1964) [21].

\section{GRAINSIZE DISTRIBUTIONS}

The only measurements to the knowledge of the author have been performed by D. A. Melford and K. R. Whittington $(1964,1965)^{6}$ $[22,43]$, using their inclusion counter; the instrument has been? discussed in section VI.B.2. Unfortunately, results have not been available to the author. They will be published in the paper of E. A. Melford and K. R. Whittington (1965) [43].

\section{FURTHER APPLICATION}

It is believed that the large field of application of structura investigations with the microprobe lies in the experimental regior: where light microscopy cannot be applied at all or only with very 
great difficulties. This would then include most of the multiphase alloy systems, where the differences between the phases present are normally too small to be measured or to be used for an exact discrimination. Therefore, etching and staining methods have to be used and must be applied very carefully to give reliable results. However, even with these methods, compositional ranges of phases (e.g., carbides, oxides, sulfides, etc.), segregations, and inhomogeneities cannot be measured stereometrically by light microscopy.

Since the probe permits the determination of the chemical composition of microvolumes, it is certainly the best signal generator for a stereometric determination. It should always be kept in mind that chemical reactions form the microstructure to be investigated and that chemical differences between phases are in most cases much more meaningful than changes in optical reflectivity. No enumeration of possibilities for application need be given; only few suggestions for very interesting experiments will be made below.

Much effort has been made to obtain a good characterization of the homogeneity of alloys, and especially of standards $(\mathrm{H}$. Yakowitz et al. 1965; H. Yakowitz et al. 1966) $[55,56]$. Stereometric methods in combination with the electron probe seem to be a very useful approach to this problem. As was pointed out in section VI.B.3., the point-count method will permit stereometric measurements even if only small segregations exist or the segregating element is present only in very low concentrations. An experiment might be performed as follows: some randomly distributed points are measured quantitatively for the elements encountered on the selected sample. Information is thereby gained about the concentration- and pulserate levels of these elements. According to the metallurgical or technological problem, appropriate "discrimination channels" are selected. If $0.5 \%$ of a certain element is present, the concentration ranges for the measurement could be:

$$
\begin{array}{llr}
\text { range I: } & < & 0.33 \% \\
\text { range II: } & 0.33-0.45 \% \\
\text { range III: } & 0.45-0.55 \% \\
\text { range IV: } & 0.55-0.67 \% \\
\text { range } \mathrm{V}: & >0.67 \%
\end{array}
$$

These limits have been chosen according to the $\left(\mathrm{C}_{\text {maximum }} / \mathrm{c}_{\text {minimum }}\right)$ ranges which could be of interest. The limits of range I and $\mathrm{V}$ 
represent a $\mathrm{C}_{\max } / \mathrm{c}_{\min }$ of 2 , whereas all segregations which are smaller than 1.2 will be registered in channel III. Channels II and IV represent medium segregations. If the coefficient of variation in the measurement should be not more than $10 \%$ (rel.), at least 100 counts have to be accumulated on each point. Therefore, the time needed for the accumulation of these 100 counts will be used for the discrimination; if a pulse rate of $10 \mathrm{c} / \mathrm{s}$ on $0.5 \%$ of this element is assumed, this would give the following scheme:

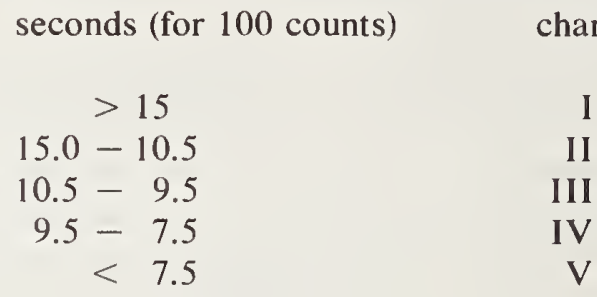

This means that, for an accumulation of 100 data points, approximately 17 minutes will be needed. If a higher counting rate can be obtained, the time will be shorter. The distance between the points can be selected according to the mean distance of the segregations. For good statistical accuracy, approximately 1000 points should be analyzed. This means a total time of analysis of approximately 3 hours. To avoid drift problems, the x-ray count/beam-current ratio could be used.

By this method, the homogeneity of large areas of a sample could be tested quite accurately. Moreover, the kinetics of the homogenization can be measured as has been shown in Figure 24. While in the as-cast alloy, great amounts of the volume will be registered in ranges I, II, IV and V, the whole alloy should be within range III if the homogenization has succeeded. It should be kept in mind that the distribution of several elements can be measured simultaneously.

\section{$\mathrm{X}$. Conclusions and Suggestions}

The methods for the quantitative evaluation of microstructures have been introduced into microprobe research only recently; therefore, only relatively few applications have been published to date. 
Stereometric methods in combination with the electron microprobe seem to open a new dimension in microprobe research. In former times, scanning pictures have been considered only in a qualitative way. Now these methods allow a quantitative evaluation of the spatial distribution of phases or concentration ranges. Great emphasis should be laid on the fact that the signals of the probe,

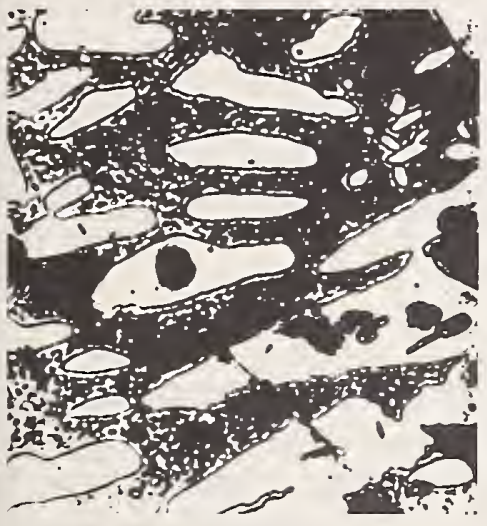

Micrograph

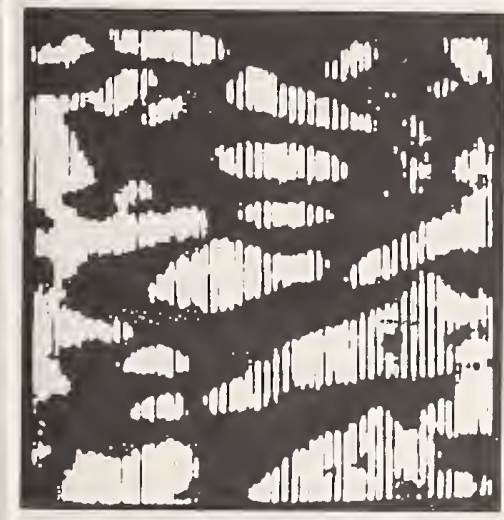

Austenite

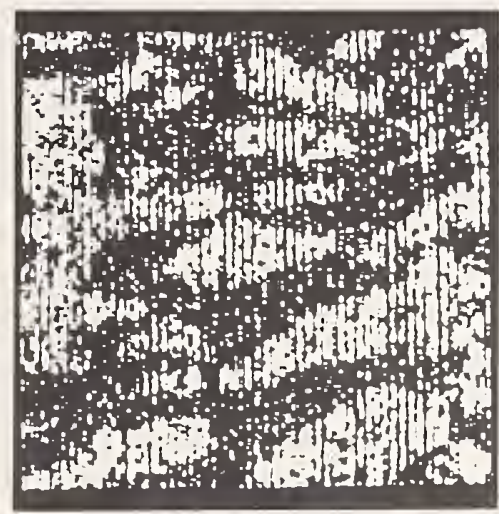

$X$-ray Scanning (expanded contrast $\mathrm{Ni}$ )

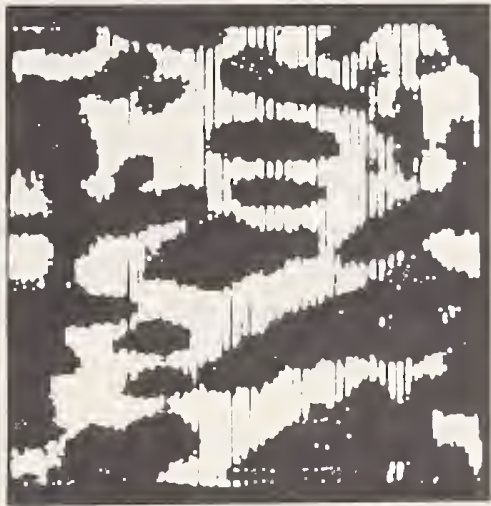

Ferrite

$100 \mu$

Figure 27. Example of the representation of phases by means of "phase images". The $\mathrm{Ni}$-concentrations used for discrimination were $8 \%$ in the ferrite and $12 \%$ in the austenite (Dörfler and Plöckinger, 1966). 
obtained during the scanning process, are analyzed directly - with no intermediate image formation. Of course, a "phase picture" can be formed simultaneously with the analyzing process. In Figure 27, the ferrite/austenite distribution in a high grade steel is shown.

In the future, these methods may help to gain considerably more insight into solid state reactions by using structural parameters which show direct correlation to the physical and chemical properties of the material investigated.

Much work should be done in the near future to develop an "ideal instrument" for stereometric analysis, which should enable the operator to perform rapid and automatic structural analysis. Some fields in which discussions and further developments should be stimulated are:

\section{A. IMPROVEMENT OF THE SIGNAL DETECTORS}

As pointed out in section $\mathrm{V}$., the emphasis lies mainly on a very sensitive signal, which makes possible an accurate discrimination between the phases present. In most cases, a high sensitivity is much more important than the possibility of obtaining quantitative results. Nondispersive $x$-ray detectors and solid-state $x$-ray detectors seem to be highly favorable. As many elements as desired may be measured simultaneously by means of several single channel analyzers. The target current amplifiers should be improved so that the detection of 0.1 of the mean atomic number is easily possible. This may be accomplished by using the "stereo monitor" to avoid topography effects and by measuring the ratio of the target current to the beam current to become independent of drift problems.

Other signal sources, such as the secondary electron emission and cathodoluminescence, should be used more extensively to obtain signal differences between the phases encountered.

\section{B. IMPROVEMENTS OF THE X-RAY INTEGRATORS}

The new "periodic pulse integrator", designed by K. F. J. Heinrich (1964) [34], has been discussed in section VI.A. It will bring considerable improvement in the spatial resolution of the method. For stereometric analyses, the integrating time should be adjustable within a wide range ( $1 \mathrm{msec}-1 \mathrm{sec})$; the integration time is selected according to the counting raté, the scanning velocity, and the statistics necessary for the discrimination. 


\section{ANALYZING CIRCUITS}

Possibilities should be provided that lineal analysis, as well as point count analysis, can be performed with the same instrument. Therefore, partially different analyzing circuits for both methods should be incorporated.

Furthermore, the analyzing circuits (including the discriminators) should be capable for a "working speed" up to approximately $100 \mathrm{kc}$ for a rapid analysis by means of the target current or the cathodoluminescence; a complete analysis by means of these signals could be performed in less than 30 seconds. According to these demands, the oscillator frequency has to be adjustable between 50 $\mathrm{c} / \mathrm{s}$ and $100 \mathrm{kc} / \mathrm{s}$. The possibility of transferring the data (intercept length or point counts) onto tape has been discussed in section VI.D.

It can be concluded that the great advantage of the system discussed above lies in the fact that large amounts of stereometric data can be collected in an objective way. The setting of the thresholds can be determined quantitatively by measuring the signal values on the encountered phases; therefore, no biased results are possible if the measurements are performed accurately. Furthermore, selective analyses are possible on phases and samples where optical methods are without any success. If these methods are combined in a suitable way with computer evaluation, numerous alloys could be investigated in order to obtain a quantitative description of the chemical and the structural composition of solid materials. As a consequence, a clear correlation between structure, chemistry, and physical properties should be observable which may lead to the programmed development of new materials.

It is hoped that this paper will stimulate some discussion and further development and that it will help in this way to reach this target within a short time.

\section{References}

[1] Fischmeister, H. F., Prakt. Metallogr. 2, 251-263 (1966).

[2] Exner, H. E., and Fischmeister, H. F., Prakt. Metallogr. 3, 18-26 (1966).

[3] Duncumb, P., and Cosslett, V. E., X-Ray Microscopy and X-Ray Microanalysis (Academic Press, New York), (1956) pp. 374-380.

[4] Rozival, A., Bull. Geol. Soc. Amer. 14, 466 (1903).

[5] Heyn, E., Metallographist 6, 37 (1903).

[6] Scheil, E., Z. Metallk. 27, 199 (1935). 
[7] Scheil, E., and Wurst, H., Z. Metallk. 28, 340-343 (1936).

[8] Smith, C.S., and Guttman, L., Trans AIME 197, 81 (1953).

[9] Saltykov, S. A., "Stereometric Metallography" (Russian) Metallurgizdat Moscow (1958).

[10] Hilliard, J. E., and Cahn, J. W., Trans AIME 221, 344-352 (I961).

[11] Elias, H., and Weibel, E., eds., "Quantitative Methods in Morphology" (Springer Verlag, Heidelberg) (1967).

[12] Underwood, E., "Quantitative Stereology" to be published (Addison-Wesley, Cambridge, Mass.) (1967).

[13] Henning, A., Zeiss Werkszeitschr. 6, 78 (1958).

[14] Dörfler, G., Thesis; University of Vienna (1966).

[15] Dörfler, G., Report at the Congress "Progress in Metallography" Leoben, Austria (1966), Radex Rundschau 3/4, 781-785 (1967).

[16] DeHoff, R. T., Trans AlME 224, 474-477 (1962).

[17] DeHoff, R. T., Trans AlME 233, 25-29 (1965).

[18] Exner, H. E., Prakt. Metallogr. 3, 334 (1966).

[19] Exner, H. E., Z. Metallk. 57, 755-764 (1966).

[20] Bockstiegel, G., Z. Metallk. 57,647 (1966).

[21] Theisen, R., Z. Metallk. 55, 128-134 (1964).

[22] Melford, D. A., and Whittington, K. R., Metallurgia 70, 49-51 (1964).

[23] Weinstein, P., Master's Thesis, Polytechn. Inst., Brooklyn, New York (1965).

[24] Dōrfler, G., Arch. Eisenhüttenwes. 36, 649-653 (1965).

[25] Heinrich, K. F. J., Advances in X-Ray Analysis Vol. 6 (Plenum Press, New York), (1962) p. 291.

[26] Melford, D. A., J. Inst. Metals 50, 217-223 (1962).

[27] Rouberol, M., et al., Mem. Sci. Rev. Met. 59, 305 (1962).

[28] Heinrich, K. F. J., NBS Tech. Note 401 (1966).

[29] von Ardenne, M., Z. Techn. Phys. 19, 407 (1938).

[30] Heinrich, K. F. J., NBS Tech. Note 278 (1967).

[31] Birks, L. S., and Batt, A. P., Anal. Chem. 35, 778-782 (1963).

[32] Keil, K., Am. Miner. 50, 2089-2092 (1965).

[33] Park, F. R., et al., Geochim. et Cosmochim. Acta 30, 399-414 (1966).

[34] Heinrich, K. F. J., Advances in X-Ray Analysis Vol. 7 (Plenum Press, New York), (1964) pp. 383-394.

[35] Malissa. H., Elektronenstrahl-Mikroanalyse (Springer Verlag Wien-New York) (1966).

[36] Duncumb, P., X-Ray Optics and X-Ray Microanalysis (Academic Press, New York), (1963) pp. 431-439.

[37] Weinryb, E., Thèse, Faculté des Sciences de l'Université de Paris.

[38] Heinrich, K. F. J., Advances in X-Ray Analysis Vol. 7 (Plenum Press. New York), (1964) pp. 325-340.

[39] Kimoto, S., and Hashimoto, H., The Electron Microprobe, McKinley et al. (ed.) (J. Wiley Sons, New York), (1966) pp. 480-489.

[40] Keil, K., Fortschr. Miner. 44 (1), 4-66 (1966).

[41] Wittry, D. B., and Kyser, D. F., J. Appl. Phys. 35, 2439 (1964).

[42] Kyser, D., and Wittry, D., The Electron Microprobe (1966), pp. 691-714.

[43] Melford, D. A., and Whittington, K. R., X-Ray Optics and X-Ray Microanalysis (Hermann, Paris, 1966) (1965), p. 497. 
[44] Moore, G. A., Photo. Sci. and Eng. 8, 152-161 (1964).

[45] Moore, G. A., NBS Report 9428 (Proc. 3rd Int. Materials Sym.) (1966).

[46] Moore, G. A., NBS Report 8073 (1963).

[47] Moore, G. A., and Wyman, L. L., Journ. Res. NBS 67A, 127-147 (1963).

[48] Ramsey, J., and Weinstein, P., The Electron Microprobe, McKinley et al., (1966) pp. 715.

[49] Dörfler, G., Report at the Congress "Metallkundliche Analyse", Vienna, 1966, Microchim. Acta, in print, (1966).

[50] Dörfler, G., Berg-und Hüttenmann, Mh. 111, 438-443 (1966).

[51] Dörfler, G., Z. Anal. Chem. 221, 357-366 (1966).

[52] Dörfler, G., Monatsh. Chem. in print, (1967).

[53] Philibert, J., and Beaulieu, C., Rev. Met. 56, 171-178 (1959).

[54] Crussard, C., Kohn, A., Beaulieu, C., and Philibert, J., Rev. Met. 56 (4), 395 406 (1959).

[55] Yakowitz, H., et al., NBS Misc. Publ, 260-10, (1965).

[56] Yakowitz, H., et al., NBS Misc. Publ. 260-12, (1966). 



\title{
SOME ASPECTS OF THE MICROPROBE ANALYSIS OF BIOLOGICAL SPECIMENS
}

\author{
T. HALL \\ Cavendish Laboratory, Cambridge, England
}

\begin{abstract}
A method suitable for the quantitative microprobe analysis of thin inhomogeneous biological specimens is presented. The intensity of a characteristic radiation is normalized against the intensity of the continuum-radiation which is simultaneously generated in the specimen. Because the method is based on ratios, it is not upset by variations in specimen thickness or density and there is no need of corrections for backscatter or "penetration". Thin standards are used, but there is no need for any standard of known thickness or mass per unit area or for any calibration mixture. Both weightfractions and changes in spatial concentrations (i.e., elemental mass per unit volume) can be measured. The method is restricted to specimens in which, on the average, the incident electrons lose only a small fraction of their energy and $\mathrm{x}$-ray self-absorption is small; for $30 \mathrm{kV}$ electrons and $\mathrm{K}$ x-rays from elements of atomic number $\mathrm{Z} \geqq 14$, these conditions are satisfied in most soft tissues sectioned freshly frozen at a thickness of $10 \mu \mathrm{m}$ or less and dried with no embeddıng material.
\end{abstract}

Other subjects which are discussed because of their special importance in biological probe work include the preservation of spatial resolution in specimens of low stopping power, the choice of operating conditions (column $\mathrm{kV}$, etc.), histological correlation, the improvement of spatial resolution, and specimen-preparation and the prevention of probe-damage. Calculations suggest that for thin specimens, high column voltages should be used. For meaningful quantitation, soft biological tissues should be prepared as simply as possible to avoid removal or displacement of elements prior to analysis. In this respect unstained sections of unembedded freshfrozen tissue seem best, but in such preparations histological correlation is difficult. The best histological correlation in unstained sections is provided perhaps by the transmission electron image available in combination electron microscope-microanalyzers, 
although there is danger of elemental loss in the procedures needed for the preparation of the very thin sections for transmission electron microscopy.

\section{Preface}

Experience has shown that probe specialists pay no attention to biological papers at probe meetings. There is mild interest when a biologist discusses technical problems common to most work, but when he talks about less familiar problems which are especially urgent for biological applications (as I shall), the audience automatically switches off. This is natural. I am bored stiff by most refinements of the conventional theory of quantitation because they are irrelevant to my problems; to follow these developments I have had to overcome a barrier which naturally also blocks attention from the other side. So I have to begin by persuading you to read this paper.

I want the paper to be read because it is high time to have expert outside consideration of the line of work which my laboratory has pursued for several years. If we are on the wrong track we had better be corrected. If we are on the right track we should be moving faster, along with more people going in the same direction.

You should read the paper because:

1. You owe it to the biologists who come to you for help. The techniques which have been developed for probe studies of minerals and metals will not take biologists very far on most of their problems.

2. While I am concerned only with biological specimens, much of the paper is applicable to the analysis of organic materials in general, and especially to the analysis of very thin specimens.

\section{Introduction}

The microprobe should be making a big contribution to biology. When a biologist hears of an instrument capable of elemental assays of amounts down to about $10^{-15}$ gms and weight-fractions down to about $10^{-4}$, with a spatial resolution around $1 \mu \mathrm{m}$, he may anticipate exciting discoveries involving the naturally occurring elements $\mathrm{Na}$, $\mathrm{Mg}, \mathrm{P}, \mathrm{S}, \mathrm{Cl}, \mathrm{K}, \mathrm{Ca}, \mathrm{Fe}, \mathrm{Cu}, \mathrm{Zn}$ and $\mathrm{I}$. In addition he can expect help in the use and evaluation of standard histochemical stains containing $\mathrm{Cr}, \mathrm{Co}, \mathrm{Ga}, \mathrm{In}, \mathrm{Pb}, \mathrm{Os}, \mathrm{U}$ and many other elements. So he is optimistic (even a bit gay) when he brings his specimen to a probe, 
generally in a department of metallurgy, mineralogy, physics or engineering, or at the applications-laboratory of a probemanufacturer.

I believe the experience is usually disappointing. In fact, with only a few exceptions, successful biological probe studies have been limited to observations of calcified tissues consisting mainly of apatite or similar minerals, and the identification of elements in foreign bodies. The reason is that the techniques developed for minerals and metals are insufficient for most biological probe work (and indeed are often inadequate for problems involving calcified tissues and foreign bodies as well). The technical difficulties are outside of the biologist's field and outside the experience of the owner of the probe. The biologist may hang on long enough to get something that he can put into a paper, but he usually decides that the promise of the probe was illusory and he had better keep to more orthodox methods in the future.

I believe that the technical problems peculiar to biological probe work are now well characterized; that solutions to most of the problems can be formulated; and that the instrumentation needed to implement these solutions already exists or is in the works. Hence the probe should be playing a much larger part in biology than is now the case. In the following sections I want first merely to specify the difficulties, then to discuss the solutions as they appear to me, and finally to summarize with a personal view of the most promising line of development.

\section{Discussion}

\section{A. A DESCRIPTION OF THE PROBLEMS}

The major problems of biological probe work are quantitation and interpretation of the $\mathrm{x}$-ray data, the preservation of spatial resolution, the correct choice of operating conditions (column $\mathrm{kV}$, probe current, etc.), histological correlation, the improvement of spatial resolution, and specimen-preparation and the prevention of probe-damage.

Quantitation and interpretation: the standard theory of staticprobe analysis assumes that the specimen is thick (i.e., opaque to the probe) and that the incident electrons and the emergent $\mathrm{x}$-rays are confined to a homogeneous region. Furthermore, the theory deals with the measurement of weight-fractions (mass of element/total mass of specimen, often called "mass concentration") but provides 
no way to measure spatial concentrations (mass of element/volume). Hence the standard theory is not suitable for soft biological tissues where one must usually be concerned with both weight-fractions and spatial concentrations, and the specimens are inhomogeneous, as well as thin in the most interesting cases. ${ }^{1}$ With few exceptions, the standard quantitative theory is applicable in biology only to calcified tissues consisting of apatite or similar minerals. Even here, local variations in density produce deceptive effects precisely in the transitional zones which are the most interesting to study. (See for example the discussion by Frazier [1] of misleading data obtained at the enamel-dentine interface in teeth.)

There is one important mitigating feature about quantitative assays of biological specimens. High accuracy is generally not wanted. Errors of 10 or even $20 \%$ are usually quite tolerable. The problem is not to be accurate, but to avoid being badly misled.

The preservation of spatial resolution: the range of a $30 \mathrm{kV}$ electron in dried soft tissue is of the order of $50 \mu \mathrm{m}$. For the preservation of a spatial resolution of the order of $1 \mu \mathrm{m}$, two approaches have been successful. One can use low-energy, shortrange electrons in the probe, or one can study thin specimens, for example ordinary histological tissue sections. These alternatives will be compared later.

Choice of operating conditions (column $k V$, etc.): for thick specimens it is well known that the analytical sensitivity depends strongly on the choice of operating conditions, and formulae predicting optimum conditions have been worked out. For thin specimens the optimum conditions are quite different, and some rough formulations for this case will be given below.

Histological and cytological correlation: ${ }^{2}$ this is one of the most serious difficulties in biological probe work. The biologist wants to measure elemental concentrations within certain components of

1 Tissues which are not calcified are called "soft", so the term encompasses the great majority of biological materials. In vivo, most soft tissues are 50-90\% water by weight. In the probe the water is gone (usually removed early in the preparative procedure and otherwise lost to the vacuum). What remains is spongy with tremendous local variation in the density of packing. It follows that one can get a good picture of the structure of a tissue only if one keeps in mind the distinction between weight-fraction and spatial concentration, and learns about both. This point will be made clear by an example later on.

${ }^{2}$ Histology is the study of tissues and cytology is the study of cells. 
tissues, such as different types of cells, intracellular organelles like nuclei and mitochondria, extracellular protein aggregates like collagen and elastin, and extracellular ground substance. But once the specimen is inside the instrument, the interesting components may not be identifiable in either the electron-scan image or the optical microscope. Optical microscopy may be quite handicapped by the presence of a heavy conducting coating.

The improvement of spatial resolution: there is no lack of important biological work for probes at a spatial resolution of one or a few $\mu \mathrm{m}$. But biologists studying ultrastructure, especially electron microscopists, rapidly lose interest in probes when they learn that the resolution is not better than $1 \mu \mathrm{m}$. (And a conventional instrument cannot offer them better than $1 \mu \mathrm{m}$. Merely to reduce the diameter of the probe, with their very thin EM sections, would result in a hopelessly low counting rate.) They will grant that $0.1 \mu \mathrm{m}$ might be quite useful, although they need $100 \AA$ or less to be really happy. These aristocrats are doing the most fundamental biological research and a considerable effort would be worthwhile if it could provide them with a resolution of $0.1 \mu \mathrm{m}$.

Specimen-preparation and the prevention of probe-damage: here I shall only remark that probe-damage is a serious danger with biological specimens but it can usually be mastered. The topic is considered in slightly more detail later.

I have separated the description of the difficulties from the discussion of methods because I believe that the foregoing brief description of the situation is not controversial, and therefore it deserves to be free from association with the methods and views which I shall now present.

\section{B. METHODS FOR BIOLOGICAL WORK}

Quantitation and interpretation: there seems to be no prospect of a theory of quantitation suitable for inhomogeneous thick specimens, but we have developed a theory which seems to be useful for measuring weight-fractions in thin biological specimens.

The weight-fraction $\mathrm{C}_{a}$ of an element a can be expressed in the form

$$
\mathrm{C}_{\mathrm{a}} \equiv \frac{\text { mass of element }}{\text { total mass }}=\frac{\text { elemental mass } / \text { area }}{\text { total mass } / \text { area }}
$$


Our approach stems from the fact that in thin specimens of soft tissue, the intensity of an element's characteristic X-radiation is approximately proportional to the local elemental mass per unit area, while the intensity of white X-radiation is approximately proportional to the local total mass per unit area. When we observe the intensity of a characteristic radiation, we simultaneously record the intensity of white $\mathrm{X}$-radiation, using a second counting system which "looks" directly at the specimen (non-diffractively) and accepts a suitable band of quantum energies. In first approximation the ratio of the two counting rates is proportional to the elemental weight-fraction.

Two versions of this scheme have been developed in my laboratory. I shall refer to them (rather inappropriately) as the "relative" method and the "absolute" method.

a. The relative method: this procedure, the more empirical of the two, was described [2] at the Washington probe conference in 1964. I shall recapitulate it here.

Given a thin specimen and a crystal spectrometer set for a characteristic line, the spectrometer counting rate $\mathrm{Y}_{a}$ may be expressed as a sum of linear contributions from the element of interest (present in mass per unit area $\mathrm{M}_{a}$ ) and from the remainder of the tissue (present in mass/area $\mathrm{M}_{m}$ ), plus a constant background $\mathrm{B}_{a}$ from the specimen-support and coating: ${ }^{3}$

$$
\mathrm{Y}_{u}=\mathrm{K}_{u a} \mathrm{M}_{a}+\mathrm{K}_{m a} \mathrm{M}_{m}+\mathrm{B}_{u}
$$

(the quantities $\mathrm{K}$ being constants of proportionality determined largely by the instrument). Similarly, the counting rate in the "white" counter, $\mathrm{Y}_{m}$, is

$$
\mathrm{Y}_{\mathrm{m}}=\mathrm{K}_{\mathrm{am}} \mathrm{M}_{\mathrm{a}}+\mathrm{K}_{\mathrm{mm}} \mathrm{M}_{\mathrm{m}}+\mathrm{B}_{\mathrm{m}} .
$$

The algebraic combination of equations (2) and (3) gives

$$
\begin{aligned}
\frac{M_{a}}{M_{m}}= & \frac{\left(Y_{a}-B_{a}\right) K_{m m}-\left(Y_{m}-B_{m}\right) K_{m a}}{\left(Y_{m}-B_{m}\right) K_{a a}-\left(Y_{a}-B_{a}\right) K_{a m}}= \\
= & \frac{\left(\frac{Y_{a}-B_{a}}{Y_{m}-B_{m}}\right)-\frac{K_{m a}}{K_{m m}} \frac{K_{m m}}{K_{a a}}-\left(\frac{Y_{a}-B_{a}}{Y_{a m}}\right) \frac{K_{m}}{K_{a m}}}{}
\end{aligned}
$$

${ }^{3}$ It is essential to use a very thin, uniform specimen-support. 
We now introduce the notations

$$
\mathrm{r} \equiv \frac{\mathrm{Y}_{\mathrm{a}}-\mathrm{B}_{\mathrm{a}}}{\mathrm{Y}_{\mathrm{m}}-\mathrm{B}_{\mathrm{m}}} ; \mathrm{r}_{\mathrm{e}} \equiv \frac{\mathrm{K}_{\mathrm{a} a}}{\mathrm{~K}_{\mathrm{am}}} ; \mathrm{r}_{\mathrm{m}} \equiv \frac{\mathrm{K}_{\mathrm{ma}}}{\mathrm{K}_{\mathrm{mm}}} .
$$

Then equation (4) can be written

$$
\frac{M_{a}}{M_{m}}=\frac{r-r_{m}}{r_{e}-r} \frac{K_{m m}}{K_{a m}} .
$$

Note that $\mathrm{r}$ is the ratio of the counts generated by the specimen in the two channels (spectrometer count/white count), $r_{e}$ is the corresponding ratio for a thin film containing only the element of interest, and $r_{m}$ is the ratio for a thin specimen of organic material containing none of the element of interest. (For pure organic material, the spectrometer count is due mainly to white radiation so close to the characteristic line that it is not rejected by the diffractor.) Thus, given thin films of the element of interest alone and of organic matrix alone, the ratios $r$ can all be measured (with due care to measure and subtract background from a supporting film when it is present and significant). These measurements and equation (5) then give a measure of $\mathbf{M}_{a} / \mathbf{M}_{m}$.

The following features of the relative method should be noted:

1. The method measures $\mathbf{M}_{a} / \mathbf{M}_{m}$ with $\mathbf{M}_{a}$ the mass of the assayed element and $\mathbf{M}_{m}$ the residual mass, i.e. the mass apart from $\mathbf{M}_{a}$ (so that the total mass is $\mathbf{M}_{a}+\mathbf{M}_{m}$ ). The weight-fraction is $\mathbf{M}_{a} /\left(\mathbf{M}_{m}+\mathbf{M}_{a}\right)$, which is nearly the same as $\mathbf{M}_{a} / \mathbf{M}_{m}$ when $\mathrm{M}_{a}<<\mathrm{M}_{m}$.

2. In the form of equation (5) the method is relative. One may compare $\mathbf{M}_{a} / \mathbf{M}_{m}$ in different spots in one or more specimens, but for an absolute measurement the instrumental factor $\mathrm{K}_{m m} / \mathrm{K}_{a m}$ must be determined. One can make the method absolute by calibrating with a thin standard containing the element of interest in known weightfraction in an organic matrix. Then both $\mathbf{M}_{a} / \mathbf{M}_{m}$ and weight-fraction $\mathrm{C}_{a}$ can be obtained absolutely. In practice the preparation of such a standard is difficult and we use the method predominantly for relative measurements.

3. For relative measurements there is no need to prepare any standard of known thickness or known mass per unit area. Furthermore, at low weight-fractions, $\mathrm{r}<<\mathrm{r}_{\mathrm{e}}$; it then follows from equation (5) that for relative measurements one does not need $r_{e}$ or the pure-element standard. Also the ratio $r_{m}$ is essentially merely the ratio of acceptance band-widths in the two channels, so that $r_{m}$ can 
be measured on almost any thin material which does not introduce a characteristic line into either channel. (We use a painted film of Aquadag.) So standardization for relative measurements is quite simple.

4. There is no restriction to small weight-fractions of the element of interest. It does not matter if the efficiency of bremsstrahlung production is different in the assayed element and in the remainder of the tissue, since these efficiencies are given separate coefficients in the formulation. However, the method does depend on a constant efficiency of bremsstrahlung production and hence on a constant average atomic number in the remainder of the tissue. This condition is quite well satisfied in most biological soft tissue (except for fat, in which $\bar{Z}$ is approximately $15 \%$ less) but the method is upset if, besides the element of interest, another heavy element is present in high concentration.

5. As a consequence of the empirical nature of the procedure, it is not necessary to exclude the characteristic line of the element of interest from the band of accepted white radiation.

6. Characteristic and white radiation are produced in parallel as the electrons dissipate their energy in the specimen. Because the method is based on the ratio of characteristic and white counting rates, the method correctly measures local variations in weightfraction in spite of variations in thickness or density of the specimens (and the results are also independent of fluctuations in intensity of the probe), and there is no need for corrections for backscatter or "penetration".

I think a description of a biological application will probably provide a better grasp of the main features of the "relative" method and will help to clear up the fog induced by the preceding paragraphs. Dr. R. T. Sims of the Department of Anatomy at Cambridge and I have been collaborating [3] on a study of the distribution of sulphur and of dry mass in layers of the skin. For present purposes the problem and the results can be represented schematically as in Figure 1.

There is a well defined boundary between the outermost part of the skin, which is a dead layer named the "corneum", and the directly underlying tissue, which is called the "viable epidermis". It was important to learn how the sulphur weight-fraction and the density of the tissue varied on the viable side of the boundary, where there is intense metabolic activity. Line scans perpendicular 


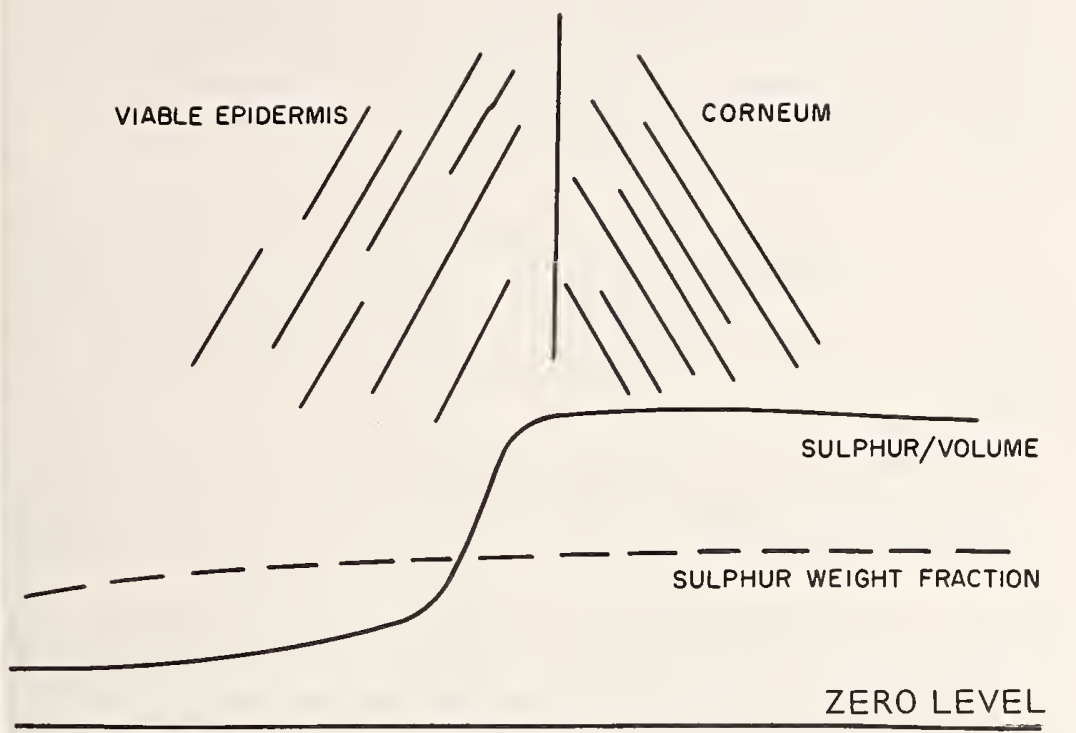

Figure 1. Schematics of sulphur distribution in skin.

to the boundary were performed on thin sections. The graph of the characteristic count rate shows the variation in amount of sulphur per unit area (solid curve in Fig. 1). The graph of the white count along the line of scan, not shown because it is quite similar to the solid curve (though not identical), depicts total mass per unit area. The dashed line is the graph of the ratio, depicting the sulphur weight-fraction.

When the section is uniform in thickness, as is normal, the curve of sulphur per unit area has the same shape as the curve of sulphur per unit volume. Thus the solid curve directly depicts the variation of spatial concentration. The normalization against the white count enables us to depict the variation of weight-fraction. We need both curves to see that at the boundary there is a steep accretion of organic dry mass (synthesis and/or packing) and hence an increase in the spatial concentration of sulphur, but no increase in sulphur weight-fraction.

b. The absolute method: this system was developed by D. J. Marshall at the Cavendish Laboratory. It was described [4] at the Paris meeting in 1965 and is discussed more fully in Marshall's recent Ph.D. thesis [5]. I shall recapitulate it here with small changes in form. 
We start from the usual expression ${ }^{4}$ for the number $\eta_{w} \mathrm{dV} d \mathrm{E}$ of white photons generated with quantum energies between $\mathrm{V}$ and $(\mathrm{V}+\mathrm{dV})$ as an electron is degraded from energy $\mathrm{E}$ to energy $(\mathrm{E}-\mathrm{dE})$ in a pure specimen of atomic number $\mathrm{Z}$ :

$$
\eta_{\mathrm{w}} \mathrm{dV} \mathrm{dE}=\mathrm{kZ} \frac{\mathrm{dV}}{\mathrm{V}} \mathrm{dE}
$$

We shall use the Bethe expression for electron energy-loss, in the form

$$
d E=\frac{2 \pi e^{4}}{E}(N Z B) d S
$$

(Here e is the electronic charge, $4.8 \times 10^{-10}$ esu; $\mathrm{N} \equiv$ atoms/cc; $\mathrm{B} \equiv \ln \mathrm{E} / \mathrm{H}$; and $\mathrm{H}$ is a function of $\mathrm{Z}$ which need not be specified more fully at this point.) For a mixture we now assume that the energy lost to atoms to type " $r$ " is given by

$$
\mathrm{dE}_{r}=\frac{2 \pi \mathrm{e}^{4}}{\mathrm{E}}\left(\mathrm{N}_{\mathrm{r}} \mathrm{Z}_{\mathrm{r}} \mathrm{B}_{\mathrm{r}}\right) \mathrm{dS} ; \mathrm{B}_{\mathrm{r}} \equiv \ln \frac{\mathrm{E}}{\mathrm{H}_{\mathrm{r}}} .
$$

We also assume ${ }^{5}$ that when an electron travels a distance dS in a mixture, the production of white photons from collisions with atoms of type $r$ is still given by

$$
\eta_{w_{r}} d V \mathrm{dE}_{r}=k Z_{r} \frac{d V}{V} \mathrm{dE}_{r},=k \frac{d V}{V} \frac{2 \pi e^{4}}{E}\left(N_{r} Z_{r}^{2} B_{r}\right) d S
$$

${ }^{4}$ While the form of equation (6) may be unfamiliar, the content is merely the usual simple approximation that the decelerating electron radiates bremsstrahlung with uniform intensity per unit frequency interval up to the cutoff frequency (cf. Compton and Allison, pages 93 and 105), and the usual approximation that the efficiency of bremsstrahlung production is proportional to atomic number (Compton and Allison, page 106).

${ }^{5}$ In equation (8) one naturally uses the value of $\mathrm{H}_{r}$ which is accepted for element $r$ in the pure state. Equations (8) and (9) are then tantamount to the assumptions that the energy of the probe-electrons is dissipated in successive independent collisions with individual independent atoms, and that the ratio of white radiation produced to energy dissipated to any one element remains the same as in the pure state. These assumptions are certainly not perfect. Chemical binding changes the energy levels of outer electrons and therefore changes the values $\mathrm{H}_{r}$ in a mixture, and the collisions are not simply with individual atoms, as shown by the existence of plasmalosses. But the end result is not affected much by the values chosen for the quantities $\mathrm{H}_{r}$. 
Then the total production of white photons from collisions with all types of atoms is obtained by simple summation and is

$$
n_{w} d V d S=k \frac{d V}{V} \frac{2 \pi e^{4}}{E}\left(\sum_{r} N_{r} Z_{r}^{2} B_{r}\right) d S,
$$

But the number of characteristic photons of a given line from atomic species a, produced in path $\mathrm{dS}$, is of the well known form

$$
\mathrm{n}_{\mathrm{a}} \mathrm{dS}=\frac{2 \pi \mathrm{e}^{4}}{\mathrm{EE}_{\mathrm{a}}} \omega \mathrm{b} \ln \frac{\mathrm{E}}{\mathrm{E}_{\mathrm{a}}} \mathrm{N}_{\mathrm{a}} \mathrm{dS}
$$

where $\mathrm{E}_{a}$ is the ionization-energy, $\omega$ is the fluorescence yield, and $\mathrm{b}$ is a constant. ${ }^{6}$ Hence the ratio of characteristic photons/white photons in a mixture, obtained by dividing (11) by (10), has the form

$$
\frac{n_{a}}{n_{w} d V}=C \frac{N_{a}}{\sum_{r} N_{r} Z_{r}^{2} B_{r}}
$$

(Here we have lumped into one constant c all the factors which are irrelevant and destined to wash away later.)

To use equation (12) in practice, measurements are made on the specimen and on one standard. The standard does not have to be a pure sample of the element of interest; it can be any thin material of known composition containing the element of interest, and there is no need to know its thickness or mass per unit area. It follows from equation (12) that

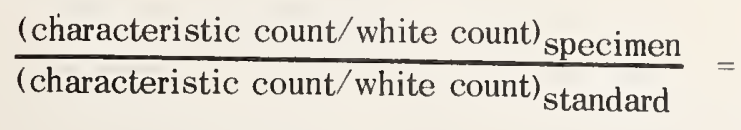

$$
\frac{\left(\frac{N_{a}}{\sum_{r} N_{r} Z_{r}^{2} B_{r}}\right)_{\text {specimen }}}{\left(\frac{N_{a}}{\sum_{r} N_{r} Z_{r}^{2} B_{r}}\right)_{\text {standard }}}
$$

${ }^{6} \mathrm{n}_{\mathrm{a}} \mathrm{ds}=\omega \sigma \mathrm{N}_{\mathrm{a}} \mathrm{ds}$, where $\sigma$ is the ionization cross section. For $\sigma \mathrm{I}$ have used

$$
\sigma=\left(2 \pi \mathrm{e}^{4} / \mathrm{EE}_{a}\right) \mathrm{b} \cdot \ln \left(\mathrm{E} / \mathrm{E}_{a}\right)
$$

This form, arising from the work of Bethe, Mott, and Massey and Worthington and Tomlin, is recommended by Green and Cosslett (Proc. Phys. Soc. 78 (1961), form (c) on page 1210). 
We should pause at this point to contemplate equation (13) because it reveals the essential simplicity of the method at the moment just before that simplicity is buried under a pile of notation and superficial complexity.

We now make the definitions

$$
\mathrm{Q} \equiv \frac{(\text { characteristic count/white count) }}{\text { specimen }}
$$

and $\quad G \equiv\left(\frac{N_{a}}{\sum_{r} N_{r} Z_{r}^{2} B_{r}}\right)$ standard

Equation (13) may now be written

$$
\mathrm{N}_{\mathrm{a}}=\mathrm{QG} \underset{\mathrm{r}}{\sum} \mathrm{N}_{\mathrm{r}} \mathrm{Z}_{\mathrm{r}}^{2} \mathrm{~B}_{\mathrm{r}}
$$

(Here and from this point on, all of the symbols with the exception of $\mathrm{Q}$ and $\mathrm{G}$ refer to the specimen alone.) It is convenient to break the sum $\Sigma$ into two parts, the term involving element $a$ plus the sum over all the other elements, which we shall denote by the symbol $\Sigma$ '.

Thus $\sum_{r} N_{r} Z_{r}^{2} B_{r}=N_{a} Z_{a}^{2} B_{a}+\sum_{r} N_{r} Z_{r}^{2} B_{r}$

By substituting (15) into (14) and solving for $\mathrm{N}_{a}$, we get

$$
N_{a}=\frac{Q G \Sigma_{r}^{\prime}\left(N_{r} Z_{r}^{2} B_{r}\right)}{1-Q G Z_{a}^{2} B_{a}}
$$

In order to put the result in terms of weight-fractions $C$, one introduces the atomic weights $\mathrm{A}$ and the relation

$$
C_{a}=\frac{N_{a} A_{a}}{\sum_{r} N_{r} A_{r}}
$$

Hence

$$
C_{a}=\frac{N_{a} A_{a}}{N_{a} A_{a}+\sum_{r} N_{r} A_{r}} \text { and } \frac{1}{C_{a}}=1+\frac{\sum_{r} N_{r} A_{r}}{N_{a} A_{a}} \text {. }
$$


The substitution of (16) into (18) gives

$$
\frac{1}{C_{a}}=1+\frac{\left(1-Q G Z_{a}^{2} B_{a}\right) \sum_{r} N_{r} A_{r}}{A_{a} Q G \sum_{r} N_{r} Z_{r}^{2} B_{r}}
$$

Equation (19) is convenient when the composition of the specimenmatrix is known directly in atom-fractions. However the compositions of soft tissue, typical protein, etc., are usually quoted in weight-fractions. To get a form which is more convenient for this case, we introduce the weight-fractions $\mathrm{C}_{r}^{\prime}$ of the various constituents in the residual matrix (i.e., exclusive of the element of interest, a):

$$
C_{r}^{\prime}=\frac{N_{r} A_{r}}{\sum_{i}^{\Sigma^{\prime} N_{i} A_{i}}} \text {, with } \Sigma_{i}^{\prime} \equiv \sum_{i \neq a}
$$

Hence

$$
N_{r}=\frac{C_{r}^{\prime}}{A_{r}} \sum_{i}^{\prime} N_{i} A_{i}
$$

The substitution of (21) into the denominator of (19) leads to the final form

$$
\frac{1}{C_{a}}=1+\frac{1-Q G Z_{a}^{2} B_{a}}{A_{a} Q G \Sigma_{r}^{\prime}\left(\frac{C_{r}^{\prime}}{A_{r}} Z_{r}^{2} B_{r}\right)} .
$$

It should be noted that if the element of interest is not normally present in the matrix, or is only a minor constituent, then the quantities $\mathrm{C}_{r}^{\prime}$ may be read out directly from literature-tables of the composition of the matrix. If the element of interest is a major constituent assumed to be present with a given weight-fraction in the tabulation, one can readily multiply the other tabulated weightfractions by a corresponding fixed factor to normalize them to the residual rather than the total mass.

Equation (22) is not nearly as inconvenient to use as it seems. Q is the only quantity in it which has to be measured. For a given element of interest, standard and probe-voltage, $G$ may be 
calculated once-and-for-all; and for a typical organic matrix and probe-voltage, the sum $\Sigma^{\prime}$ may be calculated once-and-for-all. ${ }^{7}$ For example, for the measurement of sulphur in organic matrices with 30 $\mathrm{keV}$ electrons, with a standard consisting of $\mathrm{CaSO}_{4}$ (very finely ground flakes), equation (22) reduces to

$$
\frac{1}{\mathrm{C}_{\text {Sulphur }}}=1+\frac{7.67}{\mathrm{Q}}\left(1-0.27_{6} \mathrm{Q}\right) \text {. }
$$

This is easy to use.

The absolute method permits a refinement not available with the "relative" scheme-ready extension to the case of two heavy elements (or more, in principle) in the organic matrix. For example it is important to follow the process of mineralization of tissues from the very beginning (low concentrations of $\mathrm{Ca}$ and $\mathrm{P}$ ) through intermediate stages with substantial concentrations of $\mathrm{Ca}$ and $\mathrm{P}$. As a calibration standard we use apatite, sectioned at $\leqslant 1,000 \AA$. By comparison with the $\mathrm{Ca}$ and $\mathbf{P}$ counting-rates in the standard, where $\mathrm{N}_{P} / \mathrm{N}_{c^{\prime} a}$ is known to be $3 / 5$, one can readily determine the value of the ratio $\alpha \equiv \mathrm{N}_{P} / \mathrm{N}_{c^{\prime} a}$ in the spot which is to be analyzed in the specimen. Equation (14) can be re-written in the form

$$
\begin{aligned}
N_{c a} & =Q G\left[N_{c a} Z_{c a}^{2} B_{c a}+N_{P} Z_{P}^{2} B_{P}+\Sigma^{\prime \prime} N_{r} Z_{r}^{2} B_{r}\right] \\
& =Q G\left[N_{c a}\left(Z_{c a}^{2} B_{c a}+\alpha Z_{P}^{2} B_{P}\right)+\Sigma^{\prime \prime} N_{r} Z_{r}^{2} B_{r}\right]
\end{aligned}
$$

(where $\Sigma$ " denotes the sum over all elements except Ca and P) and one then gets

$$
\frac{1}{\mathrm{C}_{\mathrm{ca}}}=1+\alpha \frac{\mathrm{A}_{\mathrm{P}}}{\mathrm{A}_{\mathrm{ca}}}+\frac{1-\mathrm{QG}\left(\mathrm{Z}_{\mathrm{ca}}^{2} \mathrm{~B}_{\mathrm{ca}}+\alpha \mathrm{Z}_{\mathrm{P}}^{\prime 2} \mathrm{~B}_{\mathrm{P}}\right)}{\mathrm{A}_{\mathrm{ca} a} \mathrm{QG} \Sigma^{\prime \prime}\left(\frac{\mathrm{C}_{\mathrm{r}}^{\prime \prime}}{\mathrm{A}_{\mathrm{r}}} \mathrm{Z}_{\mathrm{r}}^{2} \mathrm{~B}_{\mathrm{r}}\right)} .
$$

With an apatite standard and 30-keV electrons, equation (25) reduces to

$$
\frac{1}{\mathrm{C}_{\mathrm{ca}}}=1+0.78 \alpha+\frac{4.66}{\mathrm{Q}}[1-\mathrm{Q}(0.54+0.32 a)] \text {. }
$$

${ }^{7}$ One must choose one of the standard expressions for $\mathrm{B}_{r}$. The result is little affected by the choice. In the following equations I have used

$$
\mathrm{B}_{r}=\ln \left(1.17 \mathrm{E} / 11.5 \mathrm{Z}_{r}\right)(\text { with } \mathrm{E} \text { in } \mathrm{eV} .)
$$


This formula can be applied to measurements of $\mathrm{Ca}$ and $\mathrm{P}$ when both are present in elevated concentrations in tissue which is otherwise essentially a simple organic matrix.

The following features of the absolute method should be noted:

1. It is essential to exclude the characteristic line of the element of interest from the accepted band of white radiation.

2. As with the relative method, it is necessary to assume a certain composition for the remainder of the specimen aside from the element of interest, but again the method is not restricted to low weight-fractions of the element of interest.

3. Again, the procedure is not spoiled by local variations in thickness or density of the specimen or by fluctuations in the intensity of the probe, and there are no corrections to be made for backscatter or "penetration".

4. The strong point of the method, a really big advantage, is that it leads to absolute values of weight-fractions without the need to prepare calibration mixtures, or any standards of known thickness or mass per unit area at any time.

5. Uncertainties and errors: The assumptions in equations (8) and (9) have already been discussed. But the chief error in the scheme probably comes from the assumption that the bremsstrahlung-production coefficient $\mathrm{k}$ in equation (6) is independent of atomic number. There is also uncertainty about the dependence of $\mathrm{H}$ (equations 7 and 8) on atomic number, even in elements in the pure state. Marshall considers that these two factors generally might lead to probable errors of the order of $20 \%$; but in his experiments with binary mixtures, when he took $\mathrm{k}$ to be constant and used a conventional proportionality between $\mathrm{H}$ and atomic number, his overall deviations from the results of chemical assay were usually in the range $5-10 \%$. In fact it is known that $\mathrm{k}$ varies with atomic number, and the errors would presumably be less if better values of $\mathrm{k}(\mathrm{Z})$ and $\mathrm{H}(\mathrm{Z})$ were established and used. It would be easy to introduce these values into the formalism, and I believe that good experimental determinations of $k(Z)$ and $H(Z)$ would be valuable.

In collaboration with Dr. H. J. Höhling of the University of Münster and others, I am now using the absolute method in studies of $\mathrm{Ca}$, $\mathrm{P}$, and $\mathrm{S}$ at all stages of mineralization in thin sections of arteries and embryonic bone. Our early results have been reported [6] and the observations are being extended. I should not describe the work in detail here, but it will be worthwhile to mention some of the essentials. 
Among the experts there are contradictory views about the process of biological mineralization. Some consider a buildup of calcium to be the first stage while others believe that the initial step is an orderly deposition of phosphate groups onto collagen. The methods of study have been indirect and indeed most of the controversy has been supported by experiments in vitro. We have now shown by direct static-probe analyses that in arteries calcium accumulates to high levels (to weight-fractions of $1 \%$ and more) before there is a substantial accumulation of phosphorus, and we have seen a similar pattern in embryonic chick bone. I shall confine myself to a negative statement about our more recent results on embryonic rat-tooth [7] because we have not yet reported them to biologists. The pattern of mineralization in embryonic rat teeth is drastically different.

It is important to determine how the early calcium in arteries and embryonic bone is chemically bound. A short time ago Dr. A. J. Hale and I tried [8] to confirm the hypothesis of a binding of calcium to sulphated mucopolysaccharides. Mainly we studied x-ray scan images of calcium and sulphur, and the results were not very convincing. At present Höhling et al. are trying quantitative staticprobe analyses of $\mathrm{Ca}, \mathrm{P}$, and $\mathrm{S}$ and we may be able to reach a firmer conclusion.

Equipped with methods which can measure locally not only the ratios $C a: P: S$ but the weight-fractions and spatial concentrations of these elements as well, and which are sensitive enough to work right back to the really early stages ( $\mathrm{Ca}$ weight-fractions of $\sim 0.2 \%$ ), I believe we are adding substantially to the understanding of the mineralization of tissues.

Marshall's thesis [5] leaves no doubt that the quantitative method is essentially sound, and we are certainly getting reliable answers to the biological problems we are now attacking. However a huge amount remains to be done to clean up the method, both with respect to the underlying $x$-ray physical data and with respect to technique (assessment of various extraneous background effects, etc.) and it would help if other people were working along the same lines.

We have to consider how thin a specimen must be for the method to be valid. Ideally a specimen should be thin enough for the average energy-loss of the incident electrons to be very small and for $\mathrm{x}$-ray absorption to be negligible. Let us note first that so far as the measurement of weight-fractions is concerned, a large decrement in 
electron energy can actually be tolerated, because the ratio of characteristic production to white production does not change much until the ionization cross section begins to decrease (i.e., when $\mathrm{E}$ drops below $\mathrm{eE}_{K}$ ). On the other hand, for a good proportionality between the generated characteristic intensity and elemental mass per unit area, electron degradation must be more closely controlled. However even in this respect one may readily estimate that for a 30 $\mathrm{kV}$ probe, $5-\mu \mathrm{m}$ of dried soft tissue is comfortably thin and $10 \mu \mathrm{m}$ is generally acceptable.

$\mathrm{X}$-ray absorption depends on takeoff angle and above all on the penetrating power of the radiation of interest. But for example, with a takeoff angle of $20^{\circ}$ one generally does not need a correction for phosphorus-K or harder radiations in $5-\mu \mathrm{m}$ sections of soft tissue. For somewhat thicker sections, softer radiations or higher accuracy, a simple correction suffices: since the electron flux and $\mathrm{x}$-ray generation are uniform from top to bottom of a thin specimen, one may well approximate the average $x$-ray transmission by the expression

$$
\mathrm{f}=\mathrm{e}^{-(\mu / \rho)} \csc \theta(\rho \mathrm{t} / 2)
$$

The correction for weight-fractions may be considerably less than suggested by (27) since the white radiation may be similarly attenuated.

Since the biologist likes to work with tissue sections $5-\mu \mathrm{m}$ or less in thickness, our analytical method does not impose an onerous limit on thickness, at least for soft tissues. Calcified tissues must be studied in thinner form but quantitation according to our methods is still feasible, with some care, even for phosphorus- $\mathrm{K}$ radiation in apatitic specimens $1 / 2-\mu \mathrm{m}$ thick, and there is no problem at $0.1 \mu \mathrm{m}$.

We should consider a completely different means of quantitationnormalization not against white radiation but against the concentration of $\mathrm{C}, \mathrm{N}$, or $\mathrm{O}$ through the measurement of their respective $\mathrm{K}$ radiations. This method is theoretically simpler than those above and makes sense biologically, since the concentrations of $\mathrm{C}$ and $\mathrm{O}$ are fairly good indices of the concentration of organic matrix and the concentration of $\mathrm{N}$ is a good index of the concentration of protein. (The carbon background from contamination obviously raises a technical problem.) In practice I believe the high absorption of the soft $\mathrm{C}, \mathrm{N}$, and $\mathrm{O}$ radiations in specimen and conducting coating puts this method at a disadvantage. 
The preservation of spatial resolution: we have already noted that in biological tissues, to preserve spatial resolution, one must either use a short-range, low-voltage probe, or study thin specimens. If one observes only quite soft radiation (L-lines for elements like $\mathrm{Fe}$ and $\mathrm{Zn}$ ), one can use over-voltages of 3-4 and get good excitation without excessive range. This has been proved in practice by Andersen and Hasler [9]. As to the use of thin specimens, while I have not studied the matter as closely as I should, I considered it at one time with Hugh Bishop and we estimated that beam-spread is not bad for $30 \mathrm{kV}$ electrons incident on $5-\mu \mathrm{m}$ sections of soft tissue.

I have not worked at all with low probe-voltages and soft radiations and I may be prejudiced against this approach as I do not even have suitable apparatus for it. Anderson and Hasler [9] have demonstrated its high sensitivity. But I believe that in comparison with the alternative, the study of thin specimens at high voltage, the low-voltage method is disadvantageous in several respects. I shall list my doubts, perhaps unwisely as I am speaking now on the basis of no experience.

a. Reliable quantitation seems impossible.

b. To study elements in low concentrations, high probe currents are necessary with heavy conducting coatings to take away the heat. The soft radiations may be absorbed not only in the specimen but in the contamination layer and especially in the conducting coating.

c. There may be serious electron degradation and dispersion in the conducting coating.

d. If a thick specimen is used, heat-conduction is less effective than for a thin specimen coated on both sides, so that less probepower can be applied. Also, histological correlation is more difficult in thick specimens. If a thin specimen is used, low probe-voltage is no longer necessary for the preservation of resolution.

e. Elemental analysis with the soft radiations is complicated by the chemical shift of x-ray lines. (Granted, the study of chemical shifts may yield very important information about chemical states.)

Aside from these considerations, which seem generally to favor the study of thin specimens with high probe-voltages, there remains the important question of the relative sensitivity of the two approaches. This question is considered in the next section.

Choice of operating conditions (column $\mathrm{kV}$, etc.): in this section I have only estimations to offer. The calculations are tedious but the conclusions seem interesting. Therefore I shall list the conclusions 
first, before the reader is put off by the calculations. Conclusions, all relating to thin specimens: ${ }^{8}$

a. Operation at low $\mathrm{kV}$ with the detection of $\mathrm{L}$ lines should give better counting rates than operation at high $\mathrm{kV}$ with the detection of $\mathrm{K}$ lines, for the same probe current.

b. If one insists on the same resolution (same spot-size) in both cases and always puts the maximum available current into the spot, low $\mathrm{kV}$ operation should not give better counting rates.

c. If one puts maximum available current into the same spot in both cases, specimen-heating (which may well be the limiting factor) should be approximately the same for the two modes of operation.

d. A third mode of operation is to run at the higher $\mathrm{kV}$ with detection of the $\mathrm{L}$ lines. The counting rate at maximum current should be slightly greater than at low $\mathrm{kV}$, but not greater or only slightly greater than the rate for K-line detection, and the original motivation for L-line operation, the applicability of short range electrons, would of course be abandoned.

e. Background should be similar for low-voltage L-line operation and high-voltage K-line operation, or else worse at the low voltages.

I shall not speculate further about low-voltage operation but will give some additional estimates for high-voltage work, indicating that:

f. For fixed current, maximum counting rates should occur near a column voltage $\mathrm{E}_{o}=\mathrm{eE}_{k}$ ( $\mathrm{K}$-line detection, ionization energy $\mathrm{E}_{k}$, $\mathrm{e} \equiv 2.71828 \ldots$... .

g. For fixed current, optimum signal/background should occur near $\mathrm{E}_{o}=\mathrm{e}^{2} \mathrm{E}_{\kappa}$.

h. It is unrealistic to deduce optima in terms of operation at a fixed current. At higher $\mathrm{kV}$ one can put more current into a given spot and specimen-heating will still not be greater than at lower $\mathrm{kV}$. If one always puts maximum available current into a spot of fixed size, the counting rate should rise with increasing $\mathrm{E}_{o}$ with no maximum (increasing as $\ln \mathrm{E}_{o} / \mathrm{E}_{\kappa}$ ). High $\mathrm{E}_{o}$ 's are nominally even more favored if one seeks optimum signal/background. However the estimation only takes account of background generated in the specimen, and the increase of extraneous backgrounds at high $\mathrm{E}_{o}$ probably limits the height of $\mathrm{kV}$ to which one should rise.

8 More precisely, the specimens under consideration are "thin" for high-voltage electrons. They may be opaque to low-voltage probes. 
The estimations:

a. Counting rate $\mathrm{n}$ can be expressed as

$$
\mathrm{n} \propto \mathrm{i}(\bar{\phi} w) \quad(\mathrm{X} \text { A T B })
$$

where $\mathrm{i}$ is probe-current, $\bar{\phi}$ is a mean ionization cross section, $\mathrm{w} \equiv$ fluorescence yield, $\mathrm{X} \equiv$ crystal efficiency, $\mathrm{A}$ is a correction for absorption in the specimen, $\mathrm{T}$ is a correction for absorption in windows and $\mathrm{B}$ is a correction for backscatter. I shall neglect $\mathrm{A}, \mathrm{T}$, and $B$. (In fact, A and T favor the use of characteristic lines of high quantum energy and B favors the use of high-energy probeelectrons.) Also I shall assume that the probe-electrons enter the specimen with energy $\mathrm{E}_{o}=\mathrm{eE}_{\text {erige }}$ and remain at that energy, where ionization is at its peak. This assumption also is generous to the lowvoltage method, since this ideal condition can actually be maintained at high voltage but there is really substantial (total) energy loss at low voltage.

If we now assume the same current $\mathrm{i}$ and the same crystal efficiency $\mathrm{X}$ in the two cases, $(28)$ reduces to

$$
\frac{n_{L}}{n_{K}} \propto \frac{\phi_{L} W_{L}}{\phi_{K} W_{K}} \text {. }
$$

Tables indicate that $\mathrm{w}_{l} / \mathrm{w}_{\kappa} \sim 1 / 4$, roughly, and Burhop [10] indicates $\left(\phi_{l} / \phi_{k}\right) \sim 30$ for the peak ionization cross sections, so we end up with a crude estimate

$$
\mathrm{n}_{L} \sim 8 \mathrm{n}_{k}
$$

for equal-current operation. (This is probably generous again to the low-voltage method, as most often $X_{K}>X_{L}$ )

b. We need an estimate of the current which can be put into a given spot-size. The usual expression for spot-diameter $\mathrm{d}$ is

$$
d^{2}=d_{o}^{2}+d_{s}^{2}+d_{d}^{2},
$$

where $d_{o}$ is the diameter of the Gaussian image of the source, $d_{s}$ is the diameter of the disc of confusion due to spherical aberration, and $\mathrm{d}_{d}$ is the diameter corresponding to electron-diffusion. We shall neglect $\mathrm{d}_{d}$, since it should be negligible for high $\mathrm{kV}$ in a thin specimen, and we will grant the low-voltage method complete success in controlling electron-range. 
Then Duncumb's expression for spot-size [11] reduces to

$$
\mathrm{d}^{2}=\frac{\mathrm{Gi}}{\mathrm{E}_{\mathrm{o}} a^{2}}+\frac{\mathrm{S}^{2}}{4} a^{6},
$$

(with $\mathrm{G}$ a parameter of the column and gun, $\alpha$ the angular aperture and $\mathrm{S}$ the coefficient of spherical aberration).

Hence

$$
\mathrm{i}=\frac{\mathrm{n}}{(\phi \mathrm{w})(\mathrm{XATB})}=\frac{\mathrm{E}_{\mathrm{o}} a^{2}}{\mathrm{G}}\left(\mathrm{d}^{2}-\frac{\mathrm{S}^{2}}{4} a^{6}\right) .
$$

At this point, one can maximize the right-hand side of (33) and get an optimum value for the aperture for a given spot-size. In fact the optimum is $\alpha_{o p t}=(\mathrm{d} / \mathrm{S})^{1 / 3}$. However it does not matter for our purposes whether one works with optimum $\alpha$ or with fixed $\alpha$. In either case, since the optimum $\alpha$ is independent of $\mathrm{E}_{o}$ (as $\mathrm{S}$ is presumably independent of $E_{o}$ ), the expression $\left(d^{2}-\frac{s^{2}}{4} \alpha^{6}\right)$ will be the same at high and low kilovoltages. The significant result is that the available current $\mathrm{i}$ is proportional to $\mathrm{E}_{0}$. When we introduce a factor $E_{K} / E_{L}$ into (30) to convert from a fixed-current to a fixedspot comparison, we get

$$
\mathrm{n}_{L}<\mathrm{n}_{K}
$$

(constant spot-size).

c. The Bethe expression (7) suggests that heat-transfer per electron is proportional to $1 / \mathrm{E}_{o}$. (This should be a good approximation at high $\mathrm{kV}$ and at least a useful first approximation at the low $\mathrm{kV}$.) Since the current $\mathrm{i}$ varies as $\mathrm{E}_{o}$ for constant spot-size, the heating should be similar at low and high $\mathrm{kV}$.

d. The ionization cross section is known to depend on $E$ according to the form

$$
\phi \propto \frac{1}{\mathrm{E}} \ln \frac{\mathrm{E}}{\mathrm{E}_{\text {edge }}}
$$

Therefore, if $\mathrm{i}$ varies as $\mathrm{E}_{o}$ for constant spot-size, as one raises the

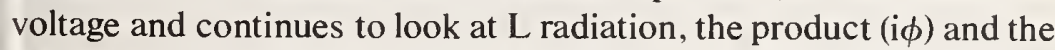
signal should increase in proportion to $\left(\ln \mathrm{E}_{o} / \mathrm{E}_{L}\right)$. But when we compare $\mathrm{K}$ and $\mathrm{L}$ counting rates at the same high $\mathrm{kV}$, the relevant factor is $\left(\phi_{L} \mathrm{~W}_{L} / \phi_{K} \mathrm{~W}_{K}\right)$. This factor was estimated to be $\leqslant 8$ when $\phi_{L}$. 
and $\phi_{K}$ were at their respective peaks, but $\phi_{L}$ is reduced by a similar factor $\left[\left(\mathrm{E}_{\text {ligh }} / \mathrm{E}_{\text {low }}\right)\left(\ln \mathrm{E}_{\text {low }} / \mathrm{E}_{l} / \ln \mathrm{E}_{\text {high }} / \mathrm{E}_{L}\right)\right]$ in going from its peak to the high $\mathrm{kV}$. Hence at the fixed high $\mathrm{kV}$, the $\mathrm{L}$ counting-rate should be less or not much more than the $\mathrm{K}$ rate.

e. For the white-spectrum background we shall start again from the expression (see equation 6)

$$
\eta_{w}(V, E) d V d E=k \bar{Z} \frac{d V}{V} d E .
$$

Equation (36) describes the white yield per probe electron. If the size of the spot is kept constant the available current is proportional to $\mathrm{E}_{o}$ and

$$
\eta_{w}^{\prime}(V, E) d V d E \propto k \bar{Z} \frac{d V}{V} E_{o} d E
$$

But the energy loss $\mathrm{dE}$ is proportional to $1 / \mathrm{E}_{\boldsymbol{\theta}}$, so

$$
\eta_{w}^{\prime}(V, E) d V \propto k \bar{Z} \frac{d V}{V} \propto \frac{d V}{V}
$$

Now

$$
\lambda=2 \mathrm{~d} \sin \theta=\frac{\mathrm{c}}{\nu}=\frac{\mathrm{hc}}{\mathrm{V}}, \text { or }
$$

$2 \mathrm{~d} \cos \theta \mathrm{d} \theta=\frac{\mathrm{hc}}{\mathrm{V}^{2}} \mathrm{dV}$, or $\frac{\mathrm{dV}}{\mathrm{V}} \propto \cot \theta \mathrm{d} \theta$.

Then the ratio of white-background counting rates for L-line and Kline detection (again neglecting factors like absorption, as above) should be

$$
\frac{\eta_{L}}{\eta_{K}} \propto \frac{\cot \theta_{L} \mathrm{~d} \theta_{L}}{\cot \theta_{K} \mathrm{~d} \theta_{K}}
$$


where $\mathrm{d} \theta_{L}$ and $\mathrm{d} \theta_{K}$ are the angular acceptance bands of the diffractors. According to published data, $\cot \theta_{L} \mathrm{~d} \theta_{L}$ and $\cot \theta_{K} \mathrm{~d} \theta_{K}$ may be fairly similar (for example, for ADP and LiF), or they may favor the $\mathrm{K}$-line detection if a less acute diffractor is used for the $\mathrm{L}$ radiation.

f. At high voltage, the K-line counting rate for a thin specimen, at constant current, will be closely proportional to $\phi_{K}$, and $\phi_{K}$ varies as $\left(1 / \mathrm{E}_{o}\right) \ln \left(\mathrm{E}_{o} / \mathrm{E}_{K}\right)$. This function has a maximum at $\mathrm{E}_{o}=\mathrm{eE}_{K}$.

g. The white background generated in the specimen, at constant current, should be proportional to $1 / \mathrm{E}_{o}$ (equation 10 ). The time $t$ required to determine a signal-rate $S$ in the presence of a background-rate $B$, to any predetermined accuracy, is known to be proportional to $\mathrm{B} / \mathrm{S}^{2}$. In this case

$$
\mathrm{t} \propto \frac{\mathrm{B}}{\mathrm{S}^{2}} \propto \frac{\mathrm{E}_{o}^{2}}{\mathrm{E}_{o}\left(\ln \mathrm{E}_{o} / \mathrm{E}_{K}\right)^{2}}=\frac{\mathrm{E}_{o}}{\left(\ln \mathrm{E}_{o} / \mathrm{E}_{K}\right)^{2}}
$$

This function has an extremum at $\mathrm{E}_{o}=\mathrm{e}^{2} \mathrm{E}_{K}$.

h. If $\mathrm{E}_{o}$ is increased with constant spot-size and with current always maximized, $i$ varies as $E_{o}$ and $d E$ varies as $1 / E_{o}$, so the heating should remain constant. The signal should vary as

$$
\mathrm{S} \propto \mathrm{E}_{o}\left(\frac{1}{\mathrm{E}_{o}} \ln \frac{\mathrm{E}_{o}}{\mathrm{E}_{K}}\right)=\ln \frac{\mathrm{E}_{o}}{\mathrm{E}_{K}} ;
$$

hence it should rise with $\mathrm{E}_{\boldsymbol{\theta}}$ like $\left(\ln \mathrm{E}_{o} / \mathrm{E}_{K}\right)$, with no maximum. The dependence of specimen-generated background on $\mathrm{E}_{o}$ should be $\mathrm{B}$ varies as $E_{o}\left(1 / E_{o}\right) \sim$ constant. Thus the figure of merit $S^{2} / B$ should have the dependence $\mathrm{S} / \mathrm{B}$ varies as $\left(\ln \mathrm{E}_{o} / \mathrm{E}_{K}\right)^{2}$, suggesting everreduced requisite counting-times as $\mathrm{E}_{0}$ is increased.

In sum, the estimations indicate that for thin specimens one should usually detect the $\mathrm{K}$ radiations and should use high column voltages $\left(\mathrm{E}_{\boldsymbol{o}}>\mathrm{e} \mathrm{E}_{K}\right)$.

I have not tested the speculations expounded above, and am not aware that they have been tested. Maybe they are all wrong. I think that experimental tests would be well worthwhile [12].

Histological and cytological correlation: the microprobe is used most naturally in biology for localization in terms of structures commensurate with the resolution of the instrument. Mammalian cells (mean diameters usually in the range 5-20 $\mu \mathrm{m}$ ), cell nuclei 
(mean diameters of a few $\mu \mathrm{m}$ ), and many other tissue-components of similar size are prime objects of attention. One wants to see these components in order to position the probe with respect to them.

The standard method for seeing these things is optical microscopy, and the standard preparation is a specimen, most often a section, 1-5 $\mu \mathrm{m}$ thick. Thicker sections are confusing because layers of cells may be superposed and the thickness may exceed the depth of focus of the microscope. In the microprobe, electron-scan images also are available, but here again a thick specimen is unfavorable (unless the probe is run at very low voltage or the image is based entirely on secondary electrons). So sections, a few $\mu \mathrm{m}$ thick, are typical objects of study.

However, even in thin sections the interesting structures are often not identifiable in electron-scan images or in the optical microscope. The optical-microscopical image of an unstained specimen is usually inadequate,${ }^{9}$ and the presence of a conducting coating may make it poorer still. Hence the problem of histological correlation is quite serious. There are several ways to deal with it.

First, there is the classical way of rendering the structures visible-stains. Those which are conventional in optical microscopy can be used in conventional probe work. In spite of the conducting coating the stains will usually at least be visible. Also there are stains containing heavy metals which will appear in the electronscan image $[13,14]$. In instruments which combine probe facilities with transmission electron microscopy, the conventional EM stains may be used. It is comforting to be able to fall back on the stains, but I want to plead that they should be avoided if at all possible. Conscientious histochemists know the doubt which clouds all observations when the specimens have previously been dragged through a series of liquid baths. They resort to stains only because they do not know how to get along without them, and they welcome a method which can work on virtually unprocessed specimens. In this respect the probe-technique has a great promise which should not be abandoned lightly. ${ }^{10}$

${ }^{9}$ Special techniques like phase and interference microscopy may provide excellent images of unstained specimens. But these two methods work best on wet material, and they are generally not available within the instrument.

101 am arguing only for restraint in ordinary staining merely for morphology. Heavymetal stains with specific stoichiometric reactions may become valuable tools for quantitation [14]. And of course there is no argument against probe investigations in which the action of the stain is itself the object of the study [13]. 
I have not had the opportunity to work with specimen-current images, or to work much with instruments which incorporate good optical microscopes. I can offer no suggestions for improving the performance of these facilities, but it is clear that such improvements would be important.

One possibility may be worth considering - bringing the quality of the backscattered-electron image closer to that achieved in good commercial scanning electron microscopes. While biologists are dismayed by the images which appear on the display-tubes of microanalyzers, they are delighted by the images produced by scanning EM's. In order to bridge the gap, I believe that the microanalyzer would not need three lenses, since spatial resolution is not at issue; perhaps the versatility of the electron-detector is the important factor.

Finally, histological correlation may be achieved by means of the transmission-EM image in the EM-MA instruments. The transmission EM-images of unstained specimens are appalling to electron-microscopists, but still they may show structure enough for one to place the probe successfully. We (Duncumb, Cooke, Höhling and myself) have had some happy experiences of this kind with embryonic (unmineralized) bone.

The transmission image in EM-MA instruments can help in two ways: By virtue of higher contrast, it may reveal gross structures which are not seen in the optical microscope even though they are quite large; and by virtue of good resolution, it may show structures important for the positioning of the probe, even though the probe may have to be much larger than the structures themselves (and it may be desired to avoid them). The latter point should make it clear that we need good resolution in EM-MA instruments intended for biological microanalysis.

The improvement of spatial resolution: resolution is limited by the decrease in available probe-current and the proportional loss of $x$ ray signal which must occur as the probe-diameter is reduced. To see how this effect may behave in practice, I have set up a simple formula and made a few estimations.

Suppose that the probe can produce $S$ counts $/ \mathrm{sec} / \mu \mathrm{g} / \mathrm{cm}^{2}$ on a thin film of an element of interest, and that the background recorded on an organic thin film is B counts $/ \mathrm{sec} / \mu \mathrm{g} / \mathrm{cm}^{2}$. Suppose that in a specimen with organic mass $\mathrm{M} \mu \mathrm{g} / \mathrm{cm}^{2}$, the element to be assayed is present only in a "hot spot", with elemental mass $\mathrm{fM} \mu \mathrm{g} / \mathrm{cm}^{2}$. (Then 
the local weight-fraction $\mathrm{C}$ is

$$
C=\frac{f M}{M+f M}=\frac{f}{1+f}
$$

The hot spot may be smaller than the probe; we define

$$
\mathrm{g} \equiv \frac{\text { hot-spot area }}{\text { probe area }}
$$

Then the following formula" enables one to estimate the weightfraction which can be assayed in running-time $\mathrm{t}$ with probable statistical error E:

$$
C=\frac{f}{1+f} \text { and } f=\frac{1}{t E^{2} M S g} \frac{1+\left(1+8 t E^{2} M B\right)^{1 / 2}}{2} .
$$

The quantities inserted into the table below have been chosen as follows: $\mathrm{M}=3 \mu \mathrm{g} / \mathrm{cm}^{2}$ : a reasonable guess for an organic section between 300 and 1,000 $\AA$ thick. S: For diffracting systems, the sensitivity of a commercial instrument for some thin films was measured and found to be in the neighborhood of $200 \mathrm{cps} / \mu \mathrm{g} / \mathrm{cm}^{2} / \mu \mathrm{a}$ of probe-current. I have assumed that $1 \mu \mathrm{a}$ of probe can be fed into a spot of diameter $\mathrm{d}=1 \mu \mathrm{m}$, so $\mathrm{S}$ is $200 \mathrm{cps} / \mu \mathrm{g} / \mathrm{cm}^{2}$ for a probe diameter of $1 \mu \mathrm{m}$. I have assumed that the available current for other diameters is (d) ${ }^{8 / 3} \mu \mathrm{a}$, so $\mathrm{S}$ for other probe-diameters is taken to be $\mathrm{S}=200 \mathrm{~d}^{8 / 3} \cdot{ }^{12} \mathrm{~B}$ is deduced from the ratio $\mathrm{S} / \mathrm{B}$ measured concurrently on the same thin film in the commercial instrument. I have taken into account the fact that the background of white radiation generated per unit mass is less in an organic matrix than in the metal films. S and B for the non-diffractive detectors: Not enough work has been done to know what can really be expected (to know, in particular, how close to the specimen the ${ }^{12}$.etector can successfully be brought). The values of $S$ in entries 7,8 , and 11 in the table may be optimistic, while entries 9,10 , and 12 are more conservative. My S/B of 50/1 may seem optimistic at first glance, but again one is aided by the low efficiency of production of white background in an organic matrix.

"Derived in the appendix at the end of this section.

12 Of course $S$ varies a great deal depending on the element and the instrument. However, in first approximation, improving $S$ by a factor $m$ will improve $d$ only by a factor $\mathrm{m}^{3 / 8}$. 
Table 1. Estimates of Sensitivity

The table gives estimates of the weight-fractions which can be measured in 20-second runs, with a probable statistical error of $20 \%$, in specimens containing a heavy element in $3 \mu \mathrm{g} / \mathrm{cm}^{2}$ of organic matrix.

\begin{tabular}{|c|c|c|c|c|c|}
\hline & $\begin{array}{l}\text { Probe } \\
\mu \mathrm{m}\end{array}$ & $\begin{array}{l}\text { Hot spot } \\
\quad \mu \mathrm{m}\end{array}$ & $\mathrm{S}$ & B & $\mathrm{C}$ \\
\hline & 1. 1 & 1 & 200 & 0.2 & 0.003 \\
\hline & $\begin{array}{ll}\text { 2. } & 1 \\
\text { 3. } & 0.32\end{array}$ & $\begin{array}{l}0.32 \\
0.32\end{array}$ & $\begin{array}{r}200 \\
10\end{array}$ & $\begin{array}{l}0.2 \\
0.01\end{array}$ & $\begin{array}{l}0.03 \\
0.04\end{array}$ \\
\hline & $\begin{array}{ll}\text { 4. } & 1 \\
5 . & 0.32 \\
6 . & 0.1\end{array}$ & $\begin{array}{l}0.1 \\
0.1 \\
0.1\end{array}$ & $\begin{array}{c}200 \\
10 . \\
0.5\end{array}$ & $\begin{array}{l}0.2 \\
0.01 \\
0.0005\end{array}$ & $\begin{array}{l}0.25 \\
0.32 \\
0.45\end{array}$ \\
\hline & 7. $\quad 0.32$ & 0.1 & 5,000 & 100. & 0.02 (optimistic?) \\
\hline & 8. 0.1 & 0.1 & 250 & 5. & $0.01(")$ \\
\hline & 9. $\quad 0.32$ & 0.1 & 1,000 & 20 & 0.04 (conservative?) \\
\hline non- & 10. 0.1 & 0.1 & 50 & 1 & $0.02(\quad " 1)$ \\
\hline & $\begin{array}{ll}\text { 11. } & 0.032 \\
12 . & 0.032\end{array}$ & $\begin{array}{l}0.032 \\
0.032\end{array}$ & $\begin{array}{r}12.5 \\
2.5\end{array}$ & $\begin{array}{l}0.25 \\
0.05\end{array}$ & $\begin{array}{l}0.06 \text { (optimistic?) } \\
0.20 \text { (conservative?) }\end{array}$ \\
\hline
\end{tabular}

(There is a slight inaccuracy for the high weight-fractions, entries 4, 5 , and 6 , where the background would come largely from the element of interest and would be higher than tabulated, as the tabulation is based on organic matrix.)

With small hot-spots one may either reduce the probe to the size of the spot or let it remain larger, and it is interesting to compare the anticipated results. The larger probe will not only give a larger total current; it will put more current onto the hot spot. On the other hand, it can only generate pure background in the surrounding tissue. When one uses a diffractor, which rejects background relatively well, the net result seems to favor the larger probe somewhat (for example, compare entries 2 and 3, or 4, 5 and 6). But with non-diffractive detection, it should pay to bring the probe right down to the size of the hot spot, in spite of the severe loss in probe current (compare 7 vs. 8 , and 9 vs. 10 ).

The estimates suggest that for biological specimens, diffractive systems will probably not be widely useful for hot spots with diameters of $0.1 \mu \mathrm{m}$. Weight-fractions of $20 \%$ or more (entries 4-6) are too rare. But I was happily surprised by the estimates for non- 
diffractive systems. Here there seems to be a real possibility of wide application to $0.1-\mu \mathrm{m}$ spots. There is even a suggestion of possible non-diffractive analysis of spots down to $0.03 \mu \mathrm{m}$ (entries 11 and 12).

Under the assumed conditions, $0.01-\mu \mathrm{m}$ hot spots would be beyond analysis. Even the non-diffractive detectors would run out of counts. Having exhausted the hypothetical possibilities of the detecting system one turns to the source, to query if more current can be put into the small spots. While "point" filaments (fieldemission or T-F) can put much more current than the older types into very small spots (less than $0.01 \mu \mathrm{m}$ ), my impression is that the point-filament sources are not radically better for spots larger than $0.01 \mu \mathrm{m}$. If so, the prospects seem poor for the probe microanalysis of tissues in spots less than $0.03 \mu \mathrm{m}$ in diameter. This is not too grievous $-0.03 \mu \mathrm{m}$ or even $0.1 \mu \mathrm{m}$ would be a splendid advance. The development seems to hinge on non-diffractive detection, and the various tactics familiar to the non-diffractive trade would have to be brought into play to take care of the well known problems of element-interferences.

In the discussion above I have failed to note that larger currents can be put into small spots by probe-forming lenses of low spherical aberration. Such lenses must have short working distances, but this might be compatible with thin specimens studied in combination electron microscope-microanalyzers. Castaing [20] pointed out in 1960 that an "ideal" lens might provide a current density approximately thirty times higher than the value I have assumed. This would make the prospects better than painted above. I am in no position to judge how much of this factor can be realized in practical designs.

Note: Derivation of equation (43).

The hot spot contains (fM) $\mu \mathrm{g} / \mathrm{cm}^{2}$ of the element of interest. If the probe is larger than the hot spot, the signal will be the same as if there were $(\mathrm{gfM}) \mu \mathrm{g} / \mathrm{cm}^{2}$ of the element of interest throughout the area of the probe. (Here I am assuming, crudely and inaccurately, that the current is uniform over the probe-spot.) Hence in time $t$ there will be (gfMSt) signal-counts and (BMt) background-counts.

Total count $=$ gfMSt + BMt

Calculated signal $=($ gfMSt $+B M t)-B M t=g f M S t$. 
Probable error in calculated signal $=[(\mathrm{gfMSt}+\mathrm{BMt})+\mathrm{BMt}]^{1 / 2}$

$$
=[\mathrm{Mt}(\mathrm{gfS}+2 \mathrm{~B})]^{1 / 2}
$$

Fractional probable error $\mathrm{E}=$

$$
\frac{[\mathrm{Mt}(\mathrm{gf} \mathrm{S}+2 \mathrm{~B})]^{1 / 2}}{\mathrm{gf} \mathrm{MSt}}=\frac{(\mathrm{gfS}+2 \mathrm{~B})^{1 / 2}}{(\mathrm{Mt})^{1 / 2} \mathrm{gf} \mathrm{S}}
$$

When this equation is solved for $\mathrm{f}$, one gets equation (43),

$$
f=\frac{1}{t E^{2} M S g} \frac{1+\left(1+8 t E^{2} M B\right)^{1 / 2}}{2}
$$

Specimen-preparation and the prevention of probe-damage: I shall not discuss the techniques of polishing, etching, etc. because I know very little about them and could only echo the literature. The preparation of the surface of hard specimens is discussed in several papers dealing with probe studies of bone and teeth [15-19,1]. A technique for the infiltration of hard tissues to produce uniform density has also been described [19].

My own laboratory is mostly concerned with the measurement of low elemental concentrations in thin sections. I shall review the preparative procedures suitable for such work.

To measure low concentrations, intense probes are needed. We use 0.1-2 $\mu \mathrm{a}$. A heavy conducting coating must be applied to remove heat-we apply 200-500 $\AA$ of Al to each surface of the thin preparation (a total of 400-1,000 \&). Background must be minimized, so we mount the sections on thin supports. Nylon film, produced in the laboratory by casting on water, has been satisfactory. At one time we used underlying EM-type grids, but we are better off without the grids, presumably because thermal gradients are less severe. The nylon is simply stretched over the open ends of $3 / 8$-inch diameter tubes.

In such preparations probe-damage is not too frequent but it sometimes occurs. If the specimen disintegrates or curls up, one may try again. A much more insidious effect is the removal of the element of interest from an apparently intact specimen. We have encountered this only rarely, but one should watch closely as the probe impinges on a field or a spot for the first time, to see if the $x$ ray count falls off from its initial value. 
As indicated earlier, the histologist must always worry about artefacts. If histologists prepared their specimens themselves, or even watched as their technicians did the job, they would not dare to believe that the final product is really like the original tissue. The sections are usually mangled, swished, stabbed, and shaken through a dozen or so baths of reagents and water. There is obviously a great danger of displacement or loss of the interesting components. If it can be managed, probe-specimens of soft tissue should be prepared simply by quick-freezing the tissue, sectioning while frozen, and drying by sublimation with no thawing. The ability to analyze such uncompromised material is one of the most important assets of the microprobe method.

\section{Summary}

Perhaps I should first summarize what I have not discussed, straightforward probe studies using only well known techniques. There is a substantial literature about the foreign stuff which gets into bodies (mostly in the lung), about accretions in various organs (in the kidney, prominently), and about the distribution of minor elements as well as $\mathrm{Ca}$ and $\mathrm{P}$ in teeth and bone. Such work is certainly useful, interesting and successful.

My own bias is towards a wider field of biological processes, both healthy and pathological. There is a good future for microprobe analysis in this wider field, but only with the help of appropriate techniques. In my opinion one must concentrate on thin specimens, put them on very thin supports, and develop a special quantitative theory for them. My guess is that the most important biological probe work will be done on thin specimens in combination EM-MA instruments, perhaps with very small spot-sizes and non-diffractive $\mathrm{x}$-ray analysis.

\section{References}

[1] Frazier, Norelco Reporter 13, 25 (Jan.-March 1966).

[2] Hall, Hale, and Switsur in The Electron Microprobe (Eds. McKinley, Heinrich, and Wittry), John Wiley and Sons, New York 1966, p. 805.

[3] Sims and Hall, J. Cell Sci., in press.

[4] Marshall and Hall in X-Ray Optics and Microanalysis (Eds. Castaing, Descamps, and Philibert), Hermann, Paris 1966, p. 374.

[5] Marshall, D. J., Ph.D. Thesis, Cavendish Laboratory, Cambridge University, 1967. Also Brit. J. Appl. Physics, in press. 
[6] Höhling, Hall, and Fearnhead, Die Naturwissenschaften 54, No. 4, 93 (1967). Höhling, Hall, Boothroyd, Cooke, Duncumb, and Fitton-Jackson, Die Naturwissenschaften 54, No. 6, 142 (1967). Höhling. Hall, Boothroyd, Cooke, Duncumb, Fearnhead, and Fitton-Jackson, Proceedings of the Conference on Calcified Tissues (meeting in Bordeaux, 1967), to be published by Springer.

[71 Hơhling, Hall, and Boyde, Die Naturwissenshaften 54, No. 23, 617 (1967).

[8] Hale, Hall, and Curran in X-Ray Optics and Microanalysis (Eds. Castaing, Descamps, and Philibert), Hermann, Paris 1966, p. 686. Hale, Hall, and Curran, J. Path. and Bact., 93, No. 1, 1 (1967).

[9] Andersen and Hasler in X-Ray Optics and Microanalysis (Eds. Castaing, Descamps, and Philibert), Hermann, Paris 1966, p. 310.

[10] Burhop, Proc. Camb. Phil. Soc. 36, 43 (1940).

[11] Duncumb, Brit. J. Appl. Physics 10, 420 (1959).

[12] Buchanan, R., has circulated an analysis somewhat similar to parts of this section in intent and contents. I do not know if his analysis has been published.

[13] Hale, J. Cell Biol. 15, 427 (1962).

14] Sims and Marshall, Nature 212, 1359 (1966).

[15] Boyde, Switsur, and Fearnhead, J. Ultrastruc. Res. 5, 201 (1961).

[16] Boyde, Switsur, and Stewart, Proc. of the 9th ORCA Congress, Pergamon Press, Oxford 1963, p. 185.

[17] Mellors; Lab. Investigation 13, 183 (1964).

18] Söremark and Grфn, Arch. Oral Biol. 11, 861 (1966).

19] Frank, Capitant, and Goni, J. Dental Research 45, 672 (1966).

20] Castaing, Adv. Electronics and Electron Physics 13, Academic Press 1960. (See pages 329,333 , and especially 335 .) 
33 

W.t.

(3)

(y)

绝

S.6. है।

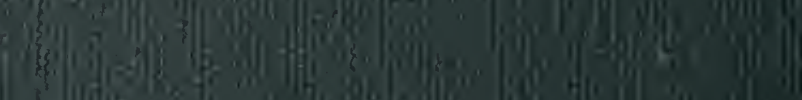

5y

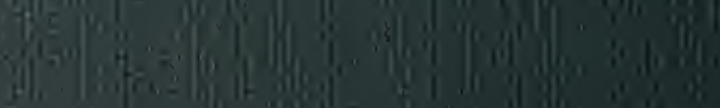

Ge. If

年

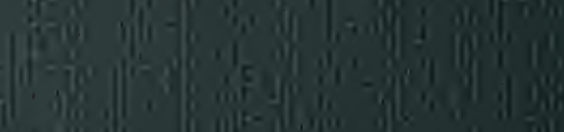

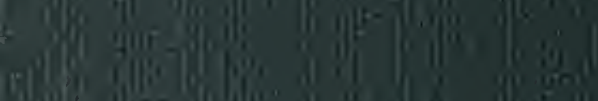

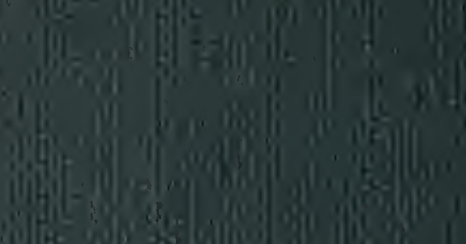

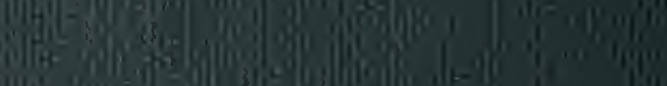

T.

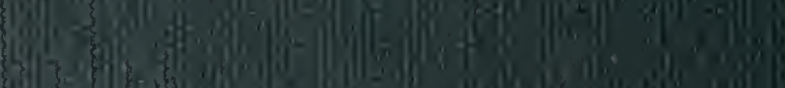

me

meth

(1)

(4)

4 